\title{
Parallel, Multigrid Finite Element Simulator for Fractured/Faulted and Other Complex Reservoirs based on Common Component Architecture (CCA)
}

\author{
Final Report
}

\section{DOE Award No. DE-FC26-04NT15531}

Period Covered by the Report: September 9, 2004 - August 31, 2008

Principal Investigator: Milind D. Deo

University of Utah

Department of Chemical and Fuels Engineering

50 South Central Campus Drive

Salt Lake City, Utah 84112

Phone: (801)581-7629

FAX: (801)585-9291

e-mail: mddeo@eng.utah.edu

\author{
Principle Authors \\ Milind D. Deo \\ Chung-Kan Huang \\ Huabing Wang
}

\section{Contributors}

Yi-kun Yang

Thomas Doe

Craig Forster

Sriram Balasubramaniam

Yao Fu

Prepared for:

U.S. Department of Energy

National Energy Technology Laboratory

February 28, 2009 


\section{Disclaimer}

This report was prepared an account of work sponsored by an agency of the United States Government. Neither the United States Government nor any agency thereof, nor any of their employees, makes any warranty, express or implied, or assumes any legal responsibility for the accuracy, completeness, or usefulness of any information, apparatus, product or process disclosed, or represents that its use would not infringe privately owned rights. Reference herein to any specific commercial product, process, or service by trade name, trademark, manufacturer, or otherwise does not necessarily constitute or imply its endorsement, recommendation, or favoring by the United States Government or any agency thereof. The views and opinions of authors expressed herein do not necessarily state or reflect those of the United States Government or any agency thereof. 


\begin{abstract}
Black-oil, compositional and thermal simulators have been developed to address different physical processes in reservoir simulation. A number of different types of discretization methods have also been proposed to address issues related to representing the complex reservoir geometry. These methods are more significant for fractured reservoirs where the geometry can be particularly challenging. In this project, a general modular framework for reservoir simulation was developed, wherein the physical models were efficiently decoupled from the discretization methods. This made it possible to couple any discretization method with different physical models.
\end{abstract}

Oil characterization methods are becoming increasingly sophisticated, and it is possible to construct geologically constrained models of faulted/fractured reservoirs. Discrete Fracture Network (DFN) simulation provides the option of performing multiphase calculations on spatially explicit, geologically feasible fracture sets. Multiphase DFN simulations of and sensitivity studies on a wide variety of fracture networks created using fracture creation/simulation programs was undertaken in the first part of this project. This involved creating interfaces to seamlessly convert the fracture characterization information into simulator input, grid the complex geometry, perform the simulations, and analyze and visualize results. Benchmarking and comparison with conventional simulators was also a component of this work. After demonstration of the fact that multiphase simulations can be carried out on complex fracture networks, quantitative effects of the heterogeneity of fracture properties were evaluated.

Reservoirs are populated with fractures of several different scales and properties. A multiscale fracture modeling study was undertaken and the effects of heterogeneity and storage on water displacement dynamics in fractured basements were investigated. In gravity-dominated systems, more oil could be recovered at a given pore volume of injection at lower rates. However, if oil production can be continued at high water cuts, the discounted cumulative production usually favors higher production rates. The workflow developed during the project was also used to perform multiphase simulations in heterogeneous, fracture-matrix systems.

Compositional and thermal-compositional simulators were developed for fractured reservoirs using the generalized framework. The thermal-compositional simulator was based on a novel "equation-alignment" approach that helped choose the correct variables to solve depending on the number of phases present and the prescribed component partitioning. The simulators were used in steamflooding and in insitu combustion applications.

The framework was constructed to be inherently parallel. The partitioning routines employed in the framework allowed generalized partitioning on highly complex fractured reservoirs and in instances when wells (incorporated in these models as line sources) were divided between two or more processors. 


\section{Table of Contents}

Abstract $1-3$

1. Executive Summary $\quad$ 1-5

2. Reservoir Simulator Framework $\quad$ 2-1

3. Simulating Heterogeneous Fracture Networks $\quad$ 3-1

4. Comparisons of DFN Models with the Homogenized Equivalents 4-1

5. Multiscale Representation of Fracture Networks and Simulation 5-1

6. Simulation of Complex Matrix-Fracture Systems $\quad$ 6-1

7. Development of the Compositional-Thermal Reservoir Simulator 7-1

8. Parallel Computation in the Framework $\quad$ 8-1

9. Generating Fracture Networks, Gridding and Creating Simulator Input Files $\quad$ 9-1 


\section{Chapter 1. Executive Summary}

An oil reservoir may undergo several recovery processes (water flooding, gas flooding, thermal recovery, etc) over its lifetime. The current simulator structure requires the use of different simulators to study each phase in the life of the simulation. Constructing a reservoir simulator using different modules will allow examination of different recovery processes using the same geologic data set and grid. Compositional and thermal simulation modules were built in this project to allow the study of compositional and thermal processes with a fully unstructured grid and with discrete fractures and faults (if, necessary). A structure to allow this type of development is also necessary. This was the first major objective of this work. The concept of modularizing the simulator revolved around decoupling the two most important components of the reservoir simulator - the discretization methods used and the physical model employed. Basic analysis concerning the solution of the differential equations for a variety of reservoir problems showed that the most effective approach is to decouple the reservoir simulator into two fundamental modules or components: discretization method (Discretization Method, DM) and physics of the reservoir model (Physical Model, PM). The DM is responsible for all the spatial related operations and properties and the governing equations are handled in the PM along with the constraint equations. A number of discretization methods, such as the conventional finite-difference, control-volume finite element, etc. were brought under the umbrella of a generalized transmissivity-based approach. Similarly, a number of physical models were incorporated. These included black-oil models to solve oil, gas and water problems, compositional models to solve carbon dioxide flooding or condensate reservoir 
problems, and thermal models to solve steam injection or in-situ combustion problems. Several sub-modules were required under each fundamental module to perform the simulation.

Most reservoirs are fractured to a certain degree, and fractures have varying degrees of impact on reservoir production. It is generally recognized that characterization of fracture networks and multiphase simulation in these networks is geologically and computationally complex. Advances in reservoir characterization are making it possible to better characterize fractures and fracture networks. It is also possible to create discrete fracture network (DFN) models and simulate multiphase flow through these networks. One of the important tasks of this project was to be able to simulate flow in a wide variety of discrete-fracture networks. There are a few commercially-available fracture generation programs, which generate fractures over a given domain. Some of these are FRACMAN and FRACA. One of the major objectives of this work was to use the fracture characterization information generated in these types of programs. Interfaces created in this project helped achieve this goal.

The reservoir simulators created in this project were validated and benchmarked with existing commercial simulators. Performances of a three-phase, black-oil reservoir simulator based on the single porosity finite difference and upstream flux weighted control volume finite element (CVFE) discretization method was evaluated on an orthogonal basement (fractured granite) fracture network. The results were in close agreement, even under three-phase flow conditions. CVFE was then used to simulate a complex network of intersecting faults that mimic a more realistic basement reservoir. CVFE simulations of the realistic network illustrate the possible consequences of uncertainty in knowing fracture/fault properties (e.g., porosity, permeability, thickness, dip orientation, connectivity and flow transmissibility). Some of the other major findings are summarized below. 
- Fracture size plays an important role in the hydrocarbon recovery process, at least in the size variations in vertical fractures/faults. It impacts the water flooding patterns and residual hydrocarbon distributions.

- Lower vertical fracture height results in higher hydrocarbon production rate during the primary production and lower hydrocarbon production rates (if producers are located on the top of fractures) during secondary production.

- If injectors and producers are linked by ultra high permeability fracture networks, limited water flooding efficiency can be expected.

- The method of using gel or blocking the main preferred pathway fracture/faults can dramatically eliminate the negative effects caused by the ultra high preferred pathways. The advantages include higher recovery rate, lower injection-water demand and lower produced water-treatment cost.

The comparison of DFN models with other conventional single-porosity, dual-porosity and homogenized models was then looked at. This study revealed some discrepancies in the current homogenization/upscaling approaches and led to the development of new method when using fine homogenized grids to represent fracture networks. An absolute permeability upscaling technique (geometric Oda method) was examined for testing the transformation from the DFN fine-grid model to a coarse-grid flow model. The single-porosity simulations were performed using ECLIPSE. The grid resolutions in the single porosity simulations were critical in capturing the geologic information from the DFN models; however, in all cases, a prespecified resolution did not produce the same result as the DFN approach. This was due to the way in which fractures intersected the grid blocks, and how the single continuum properties were attributed to the block. The new homogenization approach simplified the calculation of grid 
block properties (compared to the geometric Oda method) and produced converging fine-grid simulation results.

A study of incorporating fractures at several multiple scales was conducted to study the impact of distributing oil in different types of networks and the effect of flow rate and heterogeneity on oil recovery and water breakthrough. Basement reservoirs are often modeled using maps of faults imaged using the seismic method. Only large features are imaged and the reservoir is constructed using these trace maps. In discrete fracture network modeling, the significant fractures and faults were represented as discrete features rather than as equivalent continua. The fractures and fractures zones that are detected by seismic exploration were referred to as "seismic" fractures. However, it is known that there are more fractures within the reservoir that may contribute to both storage and connections. These undetected, but potentially significant fractures are the "subseismic" fractures. To accomplish this, DFN based conceptual seismic and subseismic basement reservoir model was constructed with two vertical parallel seismic scale features and 117 subseismic scale features with different orientations. This conceptual model could be treated as a portion of a large basement basaltic reservoir. The location of the aquifer and the nature of the aquifer support are important in waterflood performance. Water support was provided from the bottom of the seismic features in this study. Some of the conclusions of this study were:

- The reservoir performance is highly dependent on the initial oil distributions and reservoir property distributions at both the seismic/subseismic scales. Hence characterization of the basement reservoir is of paramount importance.

- The representation of subseismic scale features does have an impact on basement reservoir production behavior, if there is sufficient porosity in these features in comparison to the seismic zones. 
- At lower rate of production, higher recovery is realized for the same amount of pore volumes of water throughput. However, the only penalty of high rate production is earlier breakthrough and high water cut production. On a time basis, the higher rate option produces more oil, leading to a better discounted oil rate scenario.

The general framework created in this project allowed for the development of thermal and compositional simulators as well. In a thermal-compositional reservoir simulator, pressures, saturations, temperatures and compositions in all the existing phases must be solved. Solution of the discretized equation system implicitly requires that a system of non-linear equations is solved. A Jacobian matrix of partial derivatives is computed for this purpose. If the appropriate dependent variable is not lined-up with the correct equation, zero pivot elements are generated in the Jacobian matrix. This situation is complicated by the fact that new phases may appear, and existing phases may disappear leading to the type and number of dependent variables changing constantly. Elimination of these zero pivot elements using pivoting is computationally expensive for large matrix systems. In this project, a robust algorithm for lining-up the dependent variables and the equation system appropriately was developed. This technique can be applied to the thermal-compositional model with any number of components and phases and there are no constraints on the number of thermal equilibrium relationships. As proof of concept, two applications are discussed: a steamflooding example in a fractured system, using the discretefracture modeling approach and in situ combustion. A control volume finite-element discretization was used in steamflooding while a finite-difference approach based on the Cartesian grid was employed in the in situ combustion application.

In general, the high level of computational effort in reservoir simulations is required because of two considerations; the necessity of high resolution simulations due to the explicit description of 
detailed geological features and requirement of solving complex coupled relationships (for example, geomechanical coupling with the multiphase flow problem, and the handling of numerous components). In addition, computational requirements increase as the scale of the problem increases, and the number of control-volumes or grid blocks increase. One possible solution is parallel computing to perform the simulation on a number of processors at the same time and acquire results in a reasonable period of time. Thus, parallel computation capability becomes a necessary part of the modern reservoir simulator. The parallel computing capability is provided at the framework level, which ensures that the parallel computation capability is available no matter which DM and PM are combined later at compilation time. Currently the parallel computing routine exists as a semi-independent submodule in PM (it is physically implemented in PM but it is independent of the implementation of any PM module).

Simulating fractured reservoirs and predicting oil recovery requires integration work. The integration work should include geologic interpretation, petrophysical properties representation, well modeling and well history input. Most times, data required for this integration are scattered in various models and programs. One of the main objectives of this research program is to bring about the integration of the geologic fracture network model and reservoir simulation input file. Geometrical construction of the reservoir domain is performed manually in most cases, making the task cumbersome, particularly for complex systems. When faults and fractures are an integral part of the reservoir domain, uncertainty in creating a reliable geologic data set is higher than when the reservoir is not fractured. A workflow to go from a complex fracture model to the reservoir simulator was created in this project.

All tasks of the project were completed and some of the major project accomplishments are enumerated below. 
1. A simulation framework was created, wherein the "physical models" (black-oil, thermal and compositional) and the "discretization methods" (control-volume finite element, mixed finite element) were completely decoupled.

2. Several distinct discretization methods, (for example, the control-volume finite element and the mixed finite element) were created to study complex domains with discrete fractures and faults. The general transmissivity method used in the DM allows for the use of any control-volume based approach.

3. A methodology to grid complex, three-dimensional objects with faults and fractures was identified. The approach is to use CUBIT, the general gridding program created at Sandia National Laboratory. Using this tool and the scripts written at the University of Utah, it is now possible to take complicated faulted/fractured domains and create grids to honor the complicated geometry.

4. Three-dimensional, three-phase simulations were performed on grids generated using CUBIT. Methods to perform these computations with or without the matrix (fractureonly) simulations were developed.

5. Multiple ways of creating field-wide fracture networks were identified. The tool being used is FRED (Fractured Reservoir Development and Evaluation) created by Golder, Inc. A joint initiative with Golder was begun to perform three-phase simulations on fractured basement reservoirs, where most of the oil is stored in fractures and faults.

6. Benchmarking studies were performed on basement reservoirs, where the performance of simulators developed at the University of Utah was compared to results from other simulators such as Eclipse ${ }^{\mathrm{TM}}$. Agreement between results of simulations was excellent. 
7. A sensitivity study conducted on the fractures-only systems quantified the importance of fracture characteristics on recovery and waterflood performance.

8. A new homogenization method to convert discrete fracture maps into single porosity systems was developed.

9. Fractures at multiple scales and with significant inter-fracture heterogeneity were integrated into simulations of fractured basaltic basement systems. Simulations showed that higher rates are preferable if production at high water cuts is feasible. At the same pore volumes injection, more oil is produced at lower rates.

10. Intuitive well models of complicated wells for the mixed finite-element simulator were developed.

11. A thermal compositional simulator was developed and used to study steamflooding in fractured reservoirs, and in situ combustion processes.

12. The simulator structure was modified to use not only the conventional reservoir simulation boundary conditions (bottom hole pressure and rate constraints) but also constant flux boundary conditions and Dirichlet (constant pressure) specifications.

13. Parallelization was built into the structure of the simulator, and the effectiveness of the parallel simulator was demonstrated using the black-oil model and the thermal compositional simulator.

14. Seamless coupling with advanced numerical, computing and visualization packages (Portable Extensible Toolkit for Scientific Computing, PETSC, Trillinos, ParMetis and Paraview) was achieved in creating the simulator workflow. 


\section{Chapter 2. The Reservoir Simulator Framework}

\section{Table of Contents}

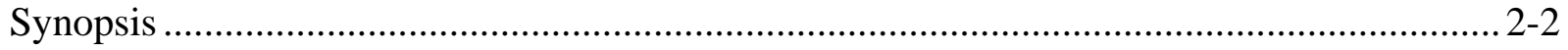

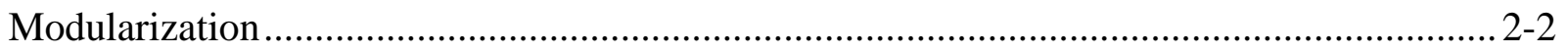

Policy in Framework.................................................................................................... 2-7

DiscretizationMethod Module (DM) ............................................................................... 2-9

PhysicalModel Module (PM)....................................................................................... 2-12

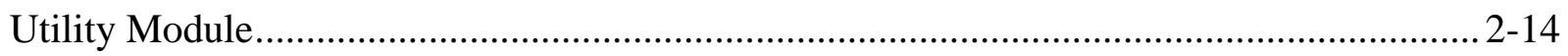

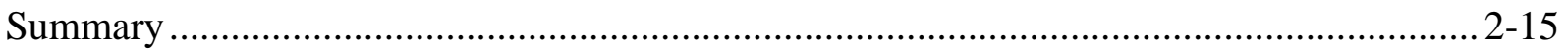




\section{Synopsis}

The merits of modularization in simulator development have been mentioned in many previous publications (for example, Yang, 2003). This chapter provides the framework design used in this research. To explain the concept effectively, several steps are needed. First, the reason for the modularization of the reservoir simulator is given. Second, the numerous programming techniques required to fulfill the modularization are introduced. The key idea in the modularization is the use of two high-level modules for performing the reservoir simulation. Finally, the content and the functionality of these two modules are discussed. Development of a utility module which provides routines that are used frequently during the coding is also discussed.

\section{Modularization}

Maximizing profit from a petroleum production operation may not be achieved by maximizing production from a single, well, reservoir or a particular process operation. One may have to consider the optimization of the entire production network which may include production from multiple reservoir, and transportation to targeted facilities. The price of the product is a complex function of supply and demand and global financial dynamics. As the ultimate goal of doing the reservoir simulation is to suggest a strategy to produce oil with better efficiency, the simulation has to consider all these aspects which are represented by several different models (Fanchi, 2006). Figure 2.1 shows the sub-models to be considered in a full-feature reservoir simulator: The reservoir model describes the multiphase/multicomponent flow within the reservoir; the well model represents fluid flow in or out of wells; the well-bore model describes the fluid flow inside the well bore (ex: flow in the tubing and casing); the surface unit model describes phase 
separation at the surface conditions; and the transportation and gathering center model can be used to evaluate the energy required and for the prediction of the final product spectrum from multiple reservoirs. Oftentimes, the produced water and gas are reinjected after cleanup. The evaluation of these recycling processes can be achieved in the surface unit model and transportation and gathering center model. A geo-mechanical model may also be necessary to include the dynamic changes in the reservoir geological properties during production. These submodels are essentially mathematical expressions so that solution of a system of partial differential equations and constraint equations (algebraic equations) is what is accomplished in a reservoir simulator.
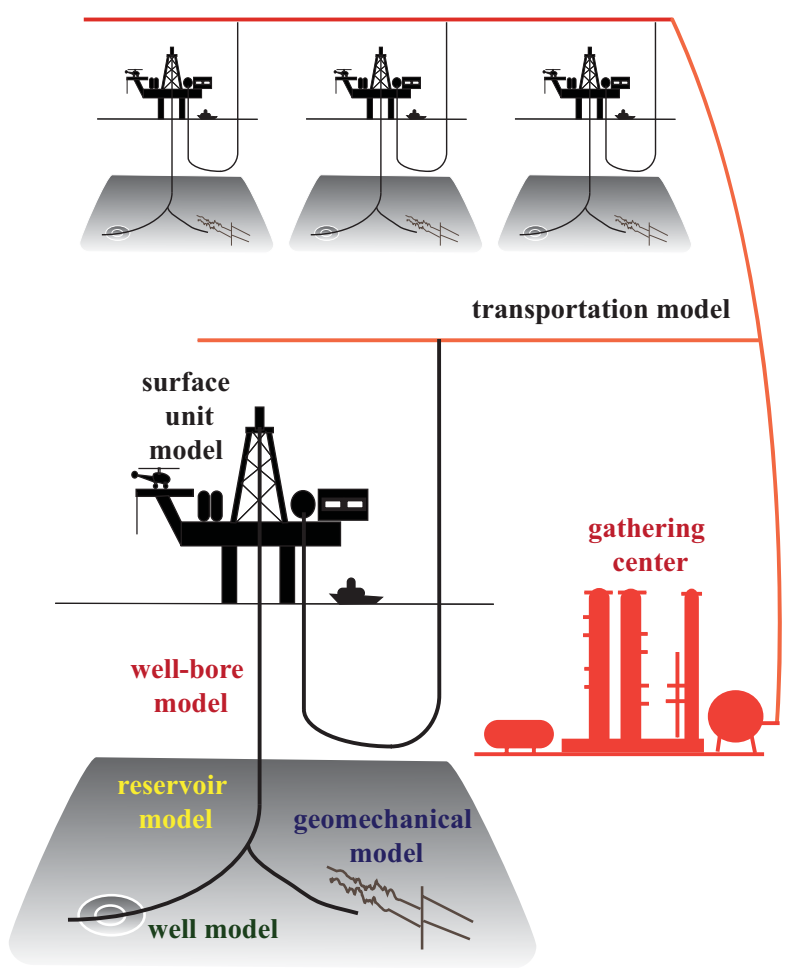

Figure 2.1 The reservoir model being shown as a sub-model of the complete production, processing and transportation system 
In the framework in this research, the reservoir model and the well model are implemented; therefore, we limit our discussion to these two models. In the reservoir model (and the geomechanical model), domain discretization is a necessary step which segments the domain into numerous small but finite sub-domains called finite volumes in order to solve the reservoir governing equations. The selection of the domain discretization method changes the way in which spatial operators and related properties (volume, depth from a datum, thickness, and the cross sectional area between control volumes) are computed. If $\mathrm{C}$ is any scalar quantity per unit volume, the conservation of this property $\mathrm{C}$ is expressed by

$$
\frac{\partial C}{\partial t}+\nabla \cdot \vec{F}_{\text {onnective }}+\nabla \cdot \vec{F}_{\text {diffusive }}=Q_{\text {source, sink }}
$$

By applying Stoke's theorem, the same equation can be expressed in an integral form for a given finite volume $v$ which has surface of A.

$$
\iiint_{\mathcal{V}}\left(\frac{\partial C}{\partial t}+Q_{\text {souroe, sink }}\right) d \mathcal{V}+\oiint_{\mathcal{A}}\left(\vec{F}_{\text {convective }}+\vec{F}_{\text {diffusive }}\right) \cdot \vec{n} d \mathcal{A}=0
$$

The discretized form of the above equation is written as:

$$
\Delta V\left(\frac{C^{t+\Delta t}-C^{t}}{\Delta t}+Q_{\text {source, sink }}\right)+\sum_{J=1}^{N_{J}}\left(\vec{F}_{\text {convective, } \mathrm{J}}+\vec{F}_{\text {diffusive, } \mathrm{J}}\right) \cdot \vec{n}_{J} \Delta A_{J}=0
$$

Convective flux and Diffusive flux of property $\mathrm{C}$ are usually obtained in the forms of

$$
\begin{aligned}
\vec{F}_{\text {convective }} & =C \vec{v} \\
\vec{F}_{\text {diffusive }} & =\kappa \rho \nabla(C / \rho)
\end{aligned}
$$

where, $\kappa$ is the diffusivity of the property of interest. 
In multiphase flow, volumetric flux v for phase p can be computed by the Darcy flow equation.

$$
\vec{v}_{p}=\overline{\overline{\mathrm{K}}} \frac{k r_{p}}{\mu_{p}}\left(\nabla P_{p}+\gamma_{p} \nabla z\right)
$$

Therefore, flow in $\mathrm{J}^{\text {th }}$ connectivity shown in the above equation can be generalized as

$$
\vec{F} \cdot \vec{n}_{J} A_{J}=\xi \overline{\bar{\tau}} \nabla \Phi \cdot \vec{n}_{J} A_{J}
$$

Where, $\xi$ is any arbitrary scalar volumetric property and $\overline{\bar{\tau}}$ and $\phi$, are the tensor and the potential of the flow respectively.

In a $N_{V}$ point linear finite element, $\phi$ can be expressed by

$$
\Phi=\sum_{\iota=1}^{N_{\mathrm{V}}} \alpha_{\iota} \Phi_{\iota}
$$

This results in the following relationship.

$$
\vec{F} \cdot \vec{n}_{J} A_{J}=\xi \overline{\bar{\tau}} \sum_{\iota=1}^{N_{\mathrm{V}}} \nabla \alpha_{\iota} \Phi_{\iota} \cdot \vec{n}_{J} A_{J}
$$

Or, in a simplified form,

$$
\vec{F} \cdot \vec{n}_{J} A_{J}=\xi \sum_{\imath=1}^{N_{\mathrm{V}}} \mathcal{T}_{\iota, J} \Phi_{\iota}
$$

where $\mathrm{T}_{l, J}$ is called transmissivity which is also determined by domain discretization.

As a result, the following computations are related to the discretization method: 
a. the volume of the finite volume

b. the transmissivities between connected finite volumes

c. the depth or elevation of the finite volume (gravitational potential)

In addition, the numbering index (identity) and the connectivity (graph) of finite volumes are also controlled by the discretization method. The scalar property $\mathrm{C}$ mentioned in the above equations is determined by the physical model used; for example, in the conventional black oil model, the scalar is the scaled volume of any phase; in compositional model, the scalar could be either mass density or molar density; in energy conservation equation, the scalar is the energy per unit volume. Once all the flux terms are computed, the completed conservation equations can be treated as algebraic equations. Several constraint equations are also required to determine the correct relationship between variables such as volume constraint and phase equilibrium. These constraint equations do not require grid information and therefore they are basically independent of the discretization method.

The above analysis shows that the most reasonable approach is to decouple the reservoir simulator into two fundamental modules or components: discretization method (DiscretizationMethod, DM) and physics of the reservoir model (PhysicalModel, PM). The DM is responsible for all the spatial related operations and properties and the governing equations are handled in the PM along with the constraint equations. Several sub-modules are required under each fundamental module to perform the simulation.

The mathematical work flow of the simulation is depicted in Figure 2.2. The figure shows that the process essentially involves solution of a set of discretized nonlinear equations, which are 
solved by the Newton's method. Time step control and choice of variables (which may have to be altered during the simulation) have to be considered for simulation efficiency. The independent development of individual module is the philosophy of framework development in this research; however, data exchange between modules is required frequently. An interface called Policy for each module has to be predefined to allow communication between modules.

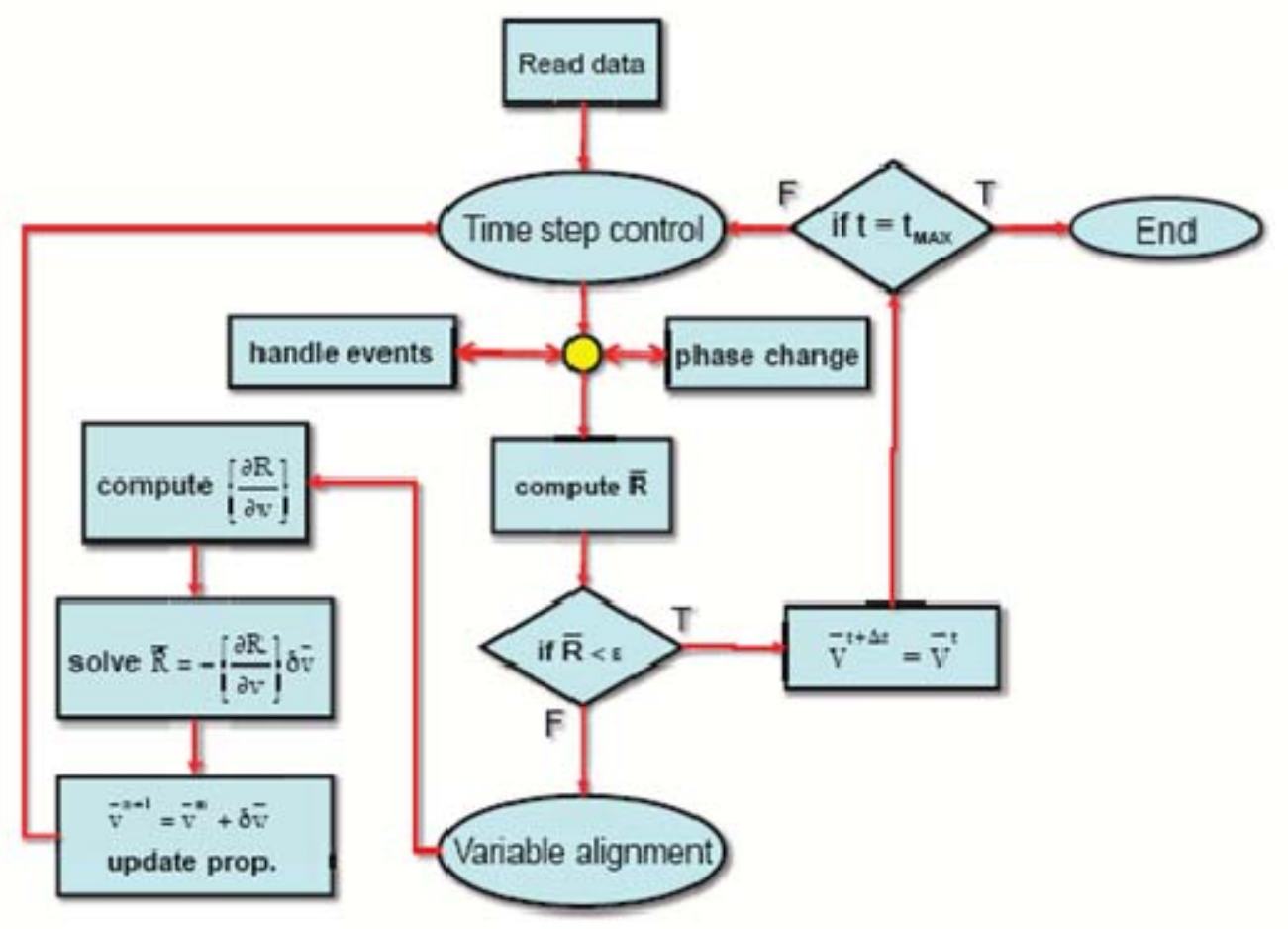

Figure 2.2 The simulation workflow showing the solution of the fully implicit equations

\section{Policy in Framework}

Policies are used for the data exchange across different modules in the framework and they are constructed using virtual functions and polymorphism concepts. A virtual function is a member function that is declared within a base class and redefined by one or more derived classes. 
Essentially, virtual functions implement the "one interface, multiple methods" philosophy that underlies polymorphism (Schildt, 2003). In our application, the virtual functions are declared as policy in high level modules and implementations are defined in lower level modules. We carefully designed the policies which can be used to represent the interfaces in the framework. Thus the policies are independent of different reservoir models or different discretization methods. For different reservoir model and discretization method applications they are implemented in derived classes of the policy classes. Figure 2.3 shows the policies defined in PM and DM and the implementations for each module under the Utah Finite Element Simulator (UFES) framework. The final simulator product which combines any DM and PM is determined at the compiling time. Detailed explanations of DM and PM are given in later sections.

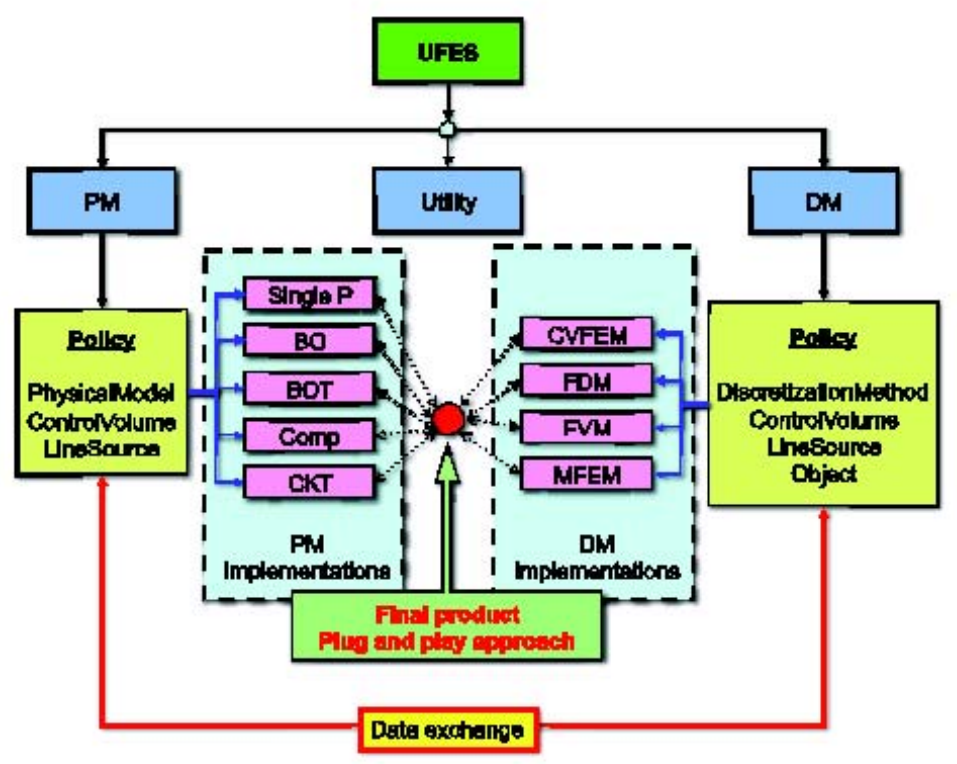

Figure 2.3 Policy implementation that makes component partitioning possible. 
A simple way to think about the relationship between the policy and the implementation is that a policy can have multiple implementations. The communications between the implementations from different modules are established through policies of each module.

\section{DiscretizationMethod Module (DM)}

The DiscretizationMethod (DM) module is responsible for computing all spatial parameters required in the flow model.

There are four policies in DM module as shown in Figure 2.4.

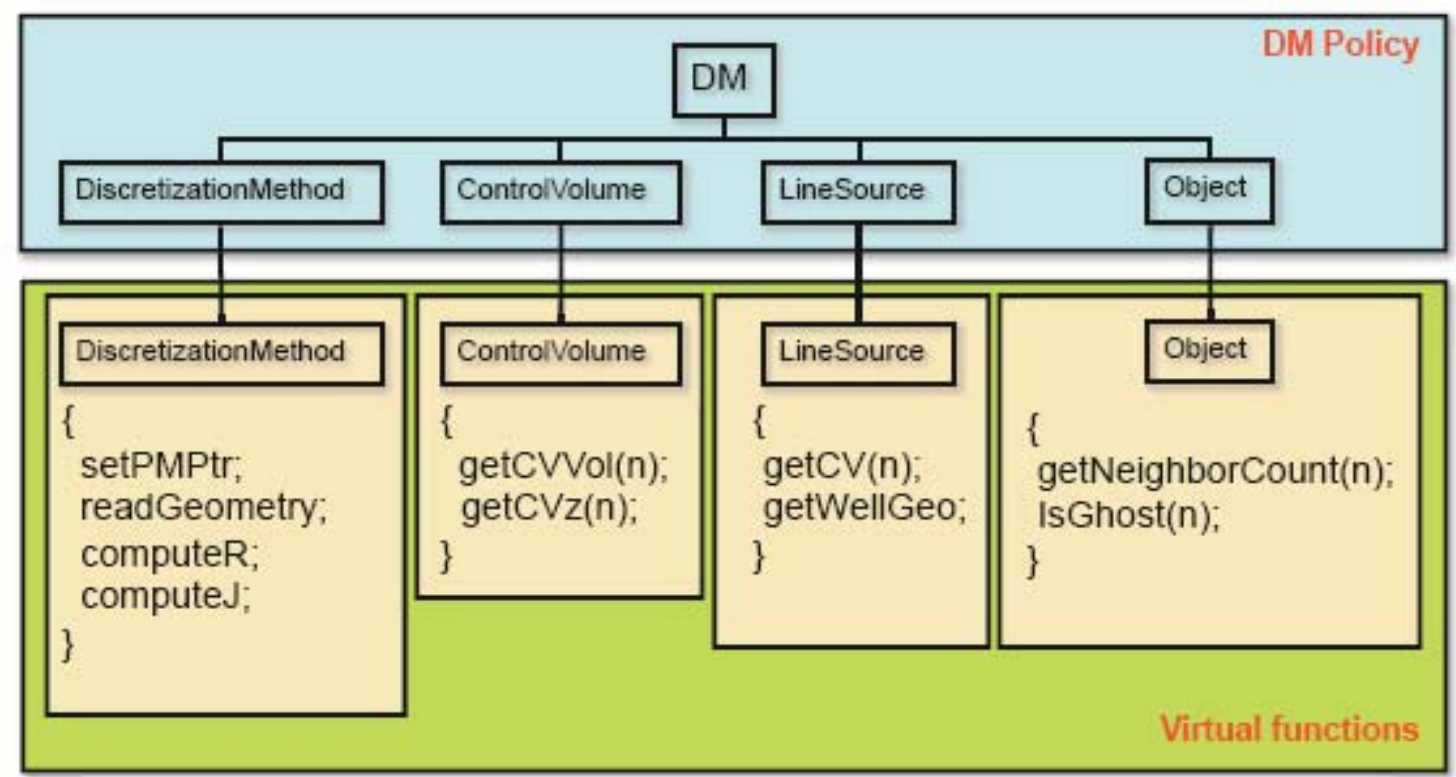

Figure 2.4 The discretization module and the associated methods.

- DiscretizationMethod : DiscretizationMethod class is the core of DM. Several essential methods ( equivalent to functions) within DiscretizationMethod class are: 
o setPMPtr: Set the PM pointer point to a PM object. DM can send and receive data through this pointer to PM whose type is determined at compilation time.

o readGeometry: The discretized domain information is read in this function. This function generates two maps: a map containing all the objects and a map containing all the connectivities. The transmissivities between objects are computed based on the given geometrical information and are stored in the connectivity map.

o computeResidualfounction: Compute residual functions. A map of all objects and another map for connectivity between objects in the domain have to be setup in DM as member data. This function call goes through the first map to call each object to compute its own residual function (for example, accumulation terms); then goes through the second map to compute the residual function corresponding to flow between objects.

o computeJacobianmatrix: Compute the Jacobian matrix. The maps mentioned above are used again, but to compute the derivatives of the residual functions with respect to the dependent variables to form the Jacobian matrix.

- ControlVolume: ControlVolume class is used to represent a finite volume. This class has methods to provide

o getCVVol(n): Provide the volume of the finite volume.

o getCVSurfaceArea(n): Provide the surface area of the finite volume. 
o getCVDz(n): Provide the thickness of the finite volume, which can be used to generate the pseudo function.

o getCVz(n): Provide the depth of the finite volume for computing the potential driving force.

- LineSource: LineSource class is used to represent a well. Methods in this class include:

o getCV(n): Find the finite volumes associated with this LineSource.

o getWellGeo: Provide the well Geometry information, which is used to calculate the well index in DM for computing production or injection rates.

- Object: Object is a unified representation of ControlVolume and LineSource, and is used to construct the graph. The methods in Object are:

o getNeighborCount(n): Provide the number of the objects connected to this Object, which the information required for most of numerical packages to assemble the Jacobian matrix.

o isGhost(n): Flag for indicating that the object IS or NOT a ghost (in parallel applications).

It can be seen that the Policy and virtual functions mentioned above in DM do not require the information specific to any type of discretization method. The methods for every virtual function are defined in the derived class of the Policy classes for the discretization method of interest. 


\section{Physical Model Module (PM)}

PM is the module for providing the properties related to the reservoir model. It's Policies and virtual functions are shown in Figure 2.5.

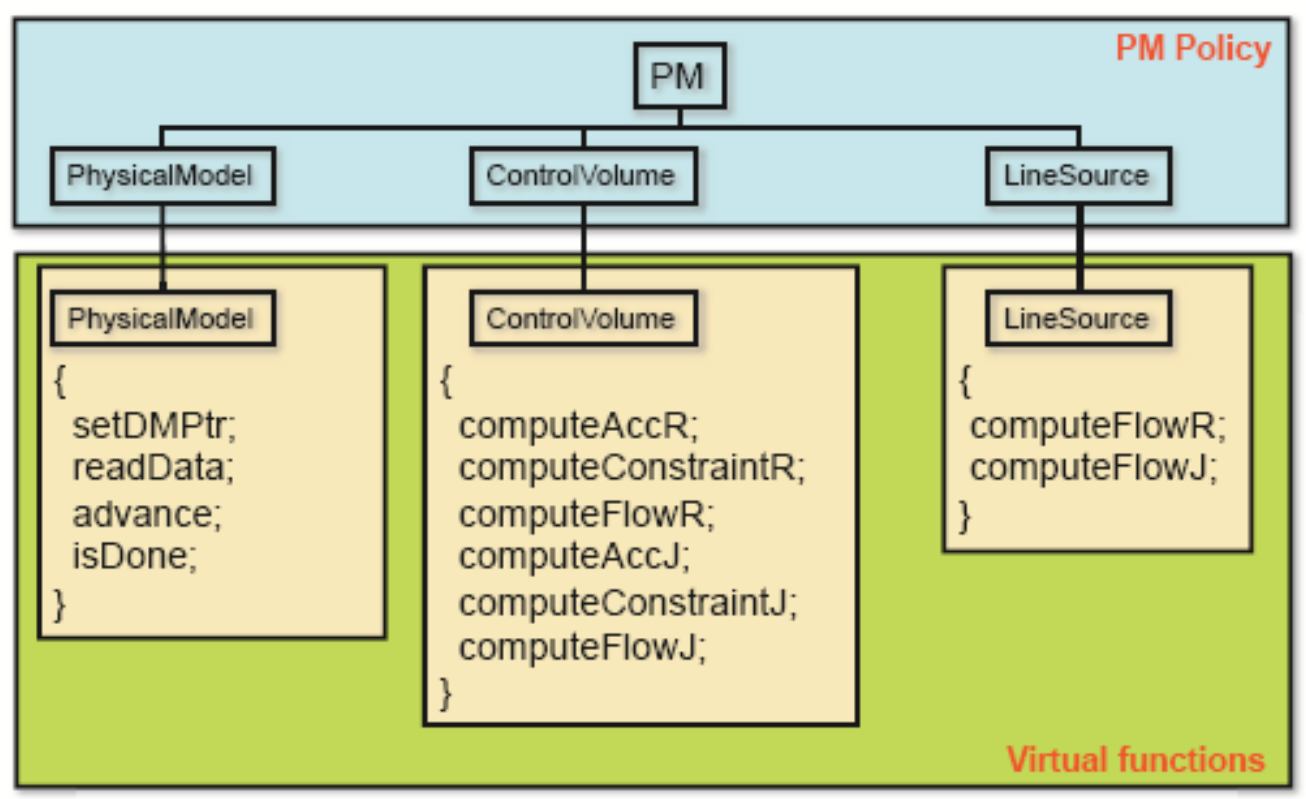

Figure 2.5 The Physical Module and associated policies and methods.

PM consists of three Policies:

- PhysicalModel: PhysicalModel class is responsible for handling operation events, control the simulation work flow and perform the non-linear iteration. The methods in this class are:

o setDMPtr: Set the DM pointer point to a DM object. PM can send and receive data through this pointer to DM whose type is determined during compilation time. 
o readData: Read the data required for the property computation. Initial conditions can be also handled in this function.

o advance: Perform non-linear iteration. Linear solver is called in this function to solve the linear system. Convergence check and time step control are also in this function. Linear system decoupling or implicit level changing can also be performed here.

o isDone: Check if the simulation has reached the last event.

- ControlVolume: ControlVolume provides the interfaces to give the results of residual functions and the derivatives of each finite volume or connectivity, which is determined by the type of reservoir model implemented.

o computeAccR: Compute residuals of accumulation terms.

o computeConstraintR: Compute residuals of constraint equations.

o computeFlowR: Compute residuals of flow terms between finite volumes.

o computeAccJ: Compute derivatives of accumulation terms.

o computeConstraintJ: Compute derivatives of constraint equations.

o computeFlowJ: Compute derivatives of flow terms between finite volumes.

- LineSource: LineSource provides the interfaces to give the results of residual functions and the derivatives of each connection between well and finite volume, which is determined by the type of the reservoir model implemented. 
o computeFlowR: Compute residuals of flow terms between finite volumes and wells.

o computeFlowJ: Compute derivatives of flow terms between finite volumes and wells.

Since the PM module is responsible for the overall simulation work flow, the master routine to assemble the global residual vector and the Jacobian matrix is implemented in PM and is shown in following pseudo code. Similar to DM, there is no specific reservoir model footprint in PM's Policies and virtual functions. The implementation of any reservoir model is defined in the derived classes of each Policy in PM.

\section{Utility Module}

This module is not directly related to any discretization method or any reservoir model. However, this module provides the tools for several operations which are common to both the modules. For example, the iterator for container of object or pointer, smart pointer, tools for linear algebra, exception handling and text parser. These utilities are often used many times in the code. In order to maximize code reuse, these utilities are coded as templates which could be either generic functions or classes. The data type within the template is parameter which can be specific once it is in use. Oftentimes, an inter-medium interface is required to convert the data for proper communication between the framework and the external library. These inter-medium interfaces can also be stored in Util module and thus can be used repeatedly by many applications created by different modules. Currently the Utility Module contains many useful 
tools such as XML parser, Epetra import / export, SmartIterator, SmartContainer, TableLookUp and Exception Handling.

\section{Summary}

This chapter described the rationale for decoupling the reservoir simulator into two high-level modules. The design concept and the architecture of the framework are also introduced. Using Policies (predefined interfaces), the implementation of each module is independent. In this research, a thermal reservoir model and three discretized methods were developed using this framework. Their development will be discussed in the next two chapters.

\section{Chapter Bibliography}

Fanchi, J.R, 2006, Principles of Applied Reservoir Simulation, Elsevier, 2006

H. Schildt, 2003, C++: The Complete Referfence, McGraw-Hil/Osborne, fourth edition, 2003.

Yang, Y.K., 2003, Finite-Element Multiphase Flow Simulation, Ph.D. Dissertation, University of Utah, 2003. 


\section{Chapter 3. Simulating Heterogeneous Fracture Networks}

\section{Table of Contents}

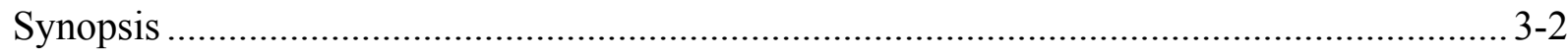

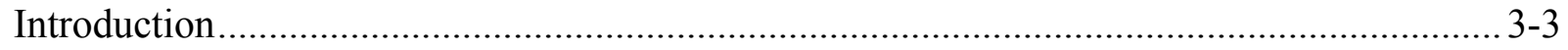

Governing Equations and DFN Model ..........................................................................

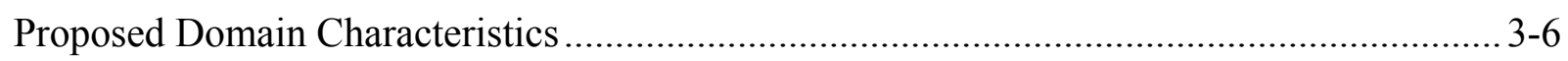

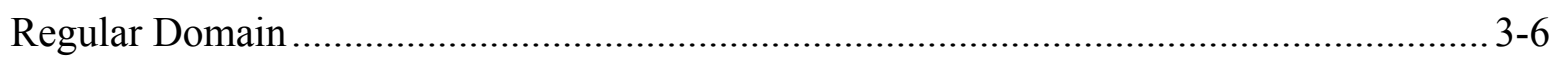

Irregular (Hypothetical "Real”) DFN Basement Domain................................................... 3-7

"Real" System and Regular "Ideal" System .................................................................. 3-8

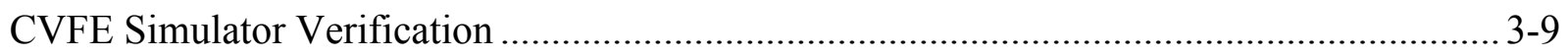

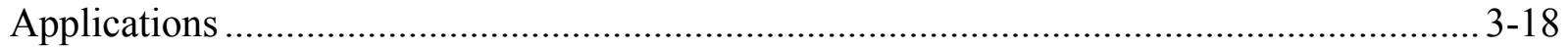

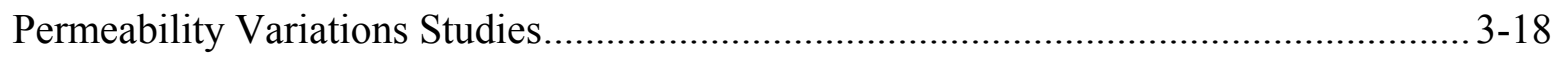

Fracture Network Connectivity Studies.................................................................... 3-27

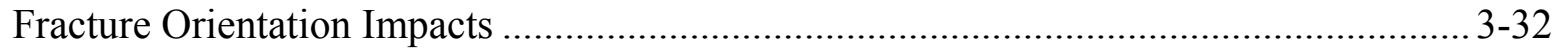

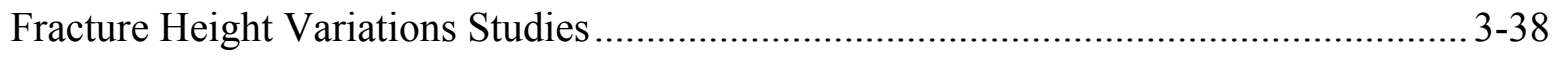

Preferred Pathway Studies .................................................................................... 3-45

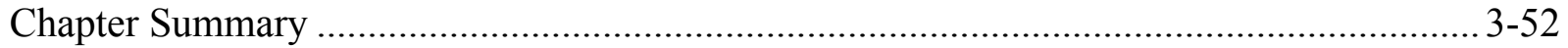

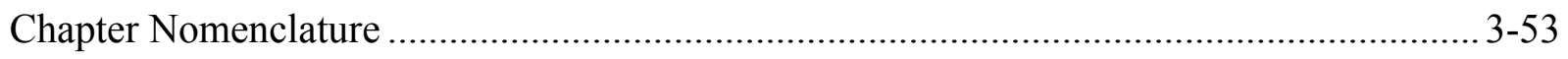

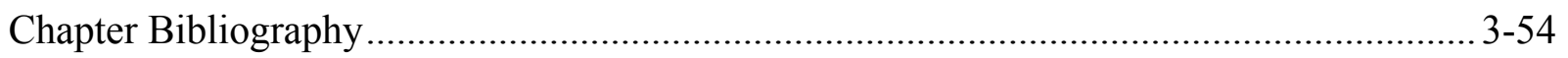




\section{Synopsis}

A fully-implicit, three-dimensional (3D), three-phase, discrete fault/fracture, black oil CVFE simulator provides new insight and understanding of oil production from reservoirs in fractured, low-permeability basement rocks. In this chapter, two three-dimensional, naturally fractured reservoirs with different fracture orientations and intensities were characterized by a discrete fracture network (DFN) geological model. Performances of three-phase, black-oil reservoir simulators based on the single porosity finite difference and upstream flux weighted CVFE discretization method were then evaluated on an orthogonal basement fracture network. Benchmark work showed that the results were in close agreement, even under three-phase flow conditions. CVFE is then used to simulate a complex network of intersecting faults that mimic a more realistic basement reservoir. The CVFE simulator provides a method that allows for complex fracture/fault geometries and spatial variations in the internal properties of faults. CVFE simulations of the realistic network illustrate the possible consequences of uncertainty in knowing fracture/fault properties (e.g., porosity, permeability, thickness, dip orientation, connectivity and flow transmissibility). For example, one of the possible consequences is the introduction of spatial variability in permeability within the fault planes (using spatially randomized patterns of 10,100 and $1000 \mathrm{md}$ ), while retaining a constant geometric mean permeability of $100 \mathrm{md}$. This enhanced oil production is due to the high-permeability pathways. This inherent variability, combined with the uncertainty of knowing the detailed connections between different regions of the fault network, impacts the pattern of sweep in ways that are important to understand when attempting to develop innovative approaches to reservoir management. 


\section{Introduction}

Quantifying fractured reservoirs is much more difficult than quantifying nonfractured reservoirs due to the extreme complexity of the fractured reservoir. The complexity originates from the vast number of uncertain variables that dictate the final fractured reservoir response. Examples of these variables and their relatives in a fractured basement reservoir include the reservoir's storage from fracture thickness and porosity; fracture permeability/transimissivity distribution characterizations; and fracture geometry such as dip orientation, connectivity, area sizes etc. The single or combined effects of those fracture variables mentioned here can make the basement network act as both permeable pathways to fluid migration and also significant storage. This brings a wide range of uncertainties for basement fractured reservoir management.

At the beginning of this chapter, a regularized fracture network was constructed that was amenable to gridding using ECLIPSE, the conventional finite difference reservoir simulator. A three-dimensional, three-phase, black oil modeled control-volume finite element (CVFE) simulator was used in this study. In order to validate the CVFE simulator, the results of regularized DFN direct simulations were compared with the results from ECLIPSE. Once the accuracy of the DFN approach was ascertained, some sensitivity of "real" basement fracture network with the multiphase DFN modeled basement reservoir was directly simulated through the CVFE simulator. Permeability distribution in the fracture/fault zone, dip orientation and thickness variations will be considered in this sensitivity study.

A typical fracture network is highly heterogeneous. To understand the impact of permeability heterogeneity between fractures, within fractures and the presence of preferred high permeability pathways between injectors and producers were quantitatively studied in this conceptual basement fractured network. The extent to which the preferred pathway network leads to early 
breakthroughs was also quantified by this chapter. The impact of shutting off the high permeability fracture using gel treatments on improving oil recovery will also be explored by this chapter.

\section{Governing Equations and DFN Model}

As shown in Equation (3-1) to (3-3), the equations describing compressible three-phase flow are obtained by combining Darcy's law and mass conservation for each phase:

$$
\begin{aligned}
& \text { OIL: } \quad-\nabla \cdot \mathbf{u}_{\theta}=\frac{\partial}{\partial t}\left(\frac{S_{Q}}{B_{Q}}\right)+q_{\theta} \\
& \text { WATER: } \quad-\nabla \cdot \mathbf{u}_{W}=\frac{\theta}{\partial \sigma}\left(\emptyset \frac{g_{W}}{\nabla_{W}}\right)+q_{W} \\
& \mathrm{GAS}: \quad-\nabla \cdot\left(R_{g} \mathbf{u}_{Q}+\mathbf{u}_{g}\right)=\frac{\partial}{\partial t}\left(\emptyset \frac{R_{g} B_{g}}{B_{Q}}+\emptyset \frac{g_{q}}{B_{g}}\right)+R_{g} q_{Q}+q_{f g}
\end{aligned}
$$

where subscript $o, w$, and $g$ represent oil, water and gas phases, $q$ is the source term, $\mathrm{R}_{\mathrm{s}}$ is the gas-oil ratio, $S$ is the saturation, $B$ is the formation volume factor. The LHS in the above equations represent the flux term, and $\mathbf{u}$ denotes the Darcy phase velocity (by combining Equation (2-5) and (2-6)).

In this three-phase black-oil model, capillary pressures coupling phase pressures are listed in Equation (3-4) and (3-5) as

$P_{e}\left(S_{w}\right)=P_{o}-P_{w}$

$P_{\theta}\left(S_{g}\right)=P_{g}-P_{Q}$

and the volume conservation equation is defined in Equation (3-6)

$s_{\sigma}+s_{w}+g_{g}=1$ 
where the subscripts $o, w$ and $g$ refer to oil, water and gas phases, $u, \rho$ and $\mu$ are the fluid velocity, density and viscosity. The rock is characterized by the porosity $\Phi$ and absolute permeability $k$. The multiphase properties are the relative permeability $k_{r}$ and capillary $p_{c} . R_{s}$ represents the gasoil ratio which is the solubility of gas in oil as a function of pressure. $B$ is the formation volume factor. Source terms are designated by $q$. The equations for three-phase flow are derived from the continuity equation: the left-hand side (LHS) is the flux term and the right-hand side (RHS) represents the cumulative term and the source term. For the basement reservoir study, all of the fluid flow through fractures is assumed to be governed by Darcy's law.

The discretization of these equations for modeling flow in basement reservoirs entails the use of the DFN approach and the CVFE scheme. In the DFN modeled three-dimensional basement domain, all of the single fractures are represented by two-dimensional surfaces and those twodimensional surfaces will be meshed into triangle elements. These dimensional reductions greatly decrease the node numbers, resulting in reduced computational time. A control volume is usually distributed across several triangular elements and calculated by summing together its subvolumes, as shown in Figure 3.1.

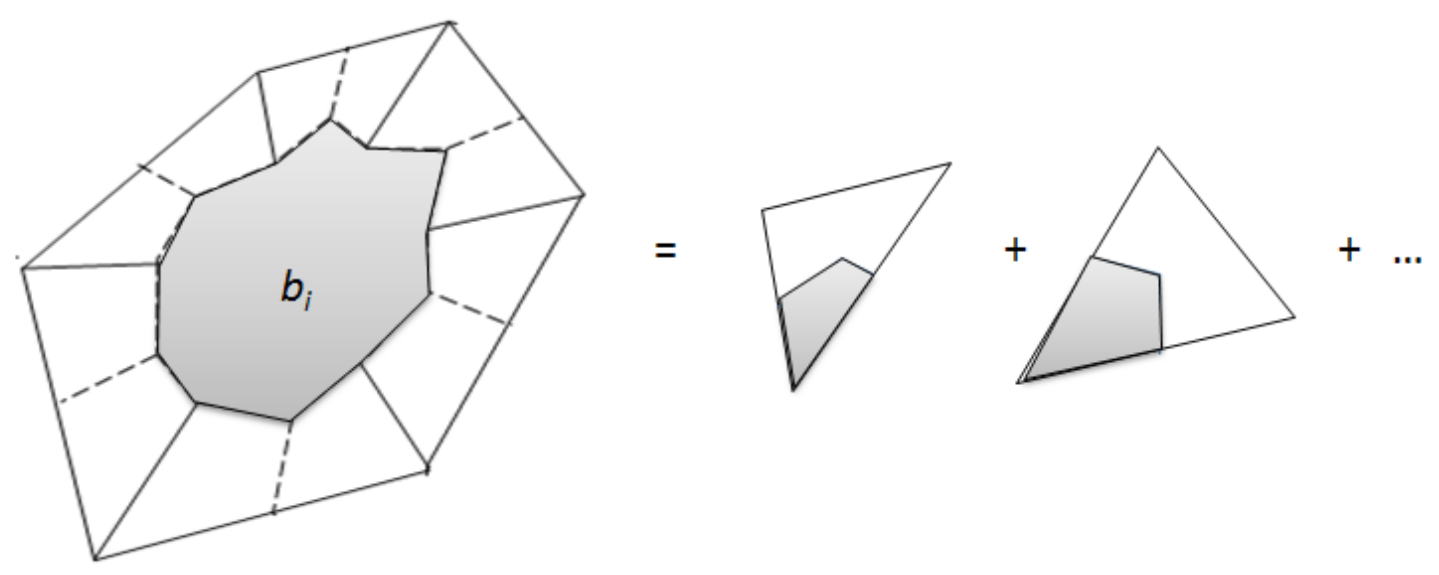

Figure 3.1 A control volume with its boundaries (dashed line) across several triangle elements and decomposition of a control volume into several subvolumes 
Figure 3.1 shows an example of a control volume with its boundaries (dashed line) across several triangle elements and decomposition of a control volume into several subvolumes. Detailed background of CVFE discretization formulation and simulator development refer to Section 2.5, the fundamentals of fractured reservoir modeling.

\section{Proposed Domain Characteristics}

\section{Regular Domain}

At the beginning of this study, a regularized well connected fracture network domain was generated for the purpose of verifying the CVFE simulator with the most popular commercial black oil simulator ECLIPSE. Since all of the features were connected orthogonally, the finitedifference based ECLIPSE simulator gave the most accurate solution. Two constraints were applied for the DFN regularized domain and its equivalent ECLIPSE single-porosity domain: the same OOIP and the same transmissivity ( $k h$ value). The regularized domain was characterized as a three-phase black oil model with initial solution gas in the oil phase. All simulations have the exact same fluid and rock properties. Examples of these properties include fluid density, viscosity, compressibility, PVT table, formation factor, gas/oil ratio table, rock compressibilities, relative permeability curves, initial conditions, well operation conditions and schedules, etc. Common model properties in the basement model are shown in Figure 3.2:

- Impermeable matrix with $\Phi=0$ (Type I, basement reservoir system)

- $\quad$ Domain $=1,000 \mathrm{ft}$ by $1,000 \mathrm{ft}$ by 200 feet deep

- Total feature length $=30,000$ feet

- Feature property: $k=1,000 \mathrm{md}, \Phi=14 \%$, width $=0.5$ feet

- Original Oil In Place (OOIP) $=53,580$ STB

- Injection Pressure $=4,300$ psi 

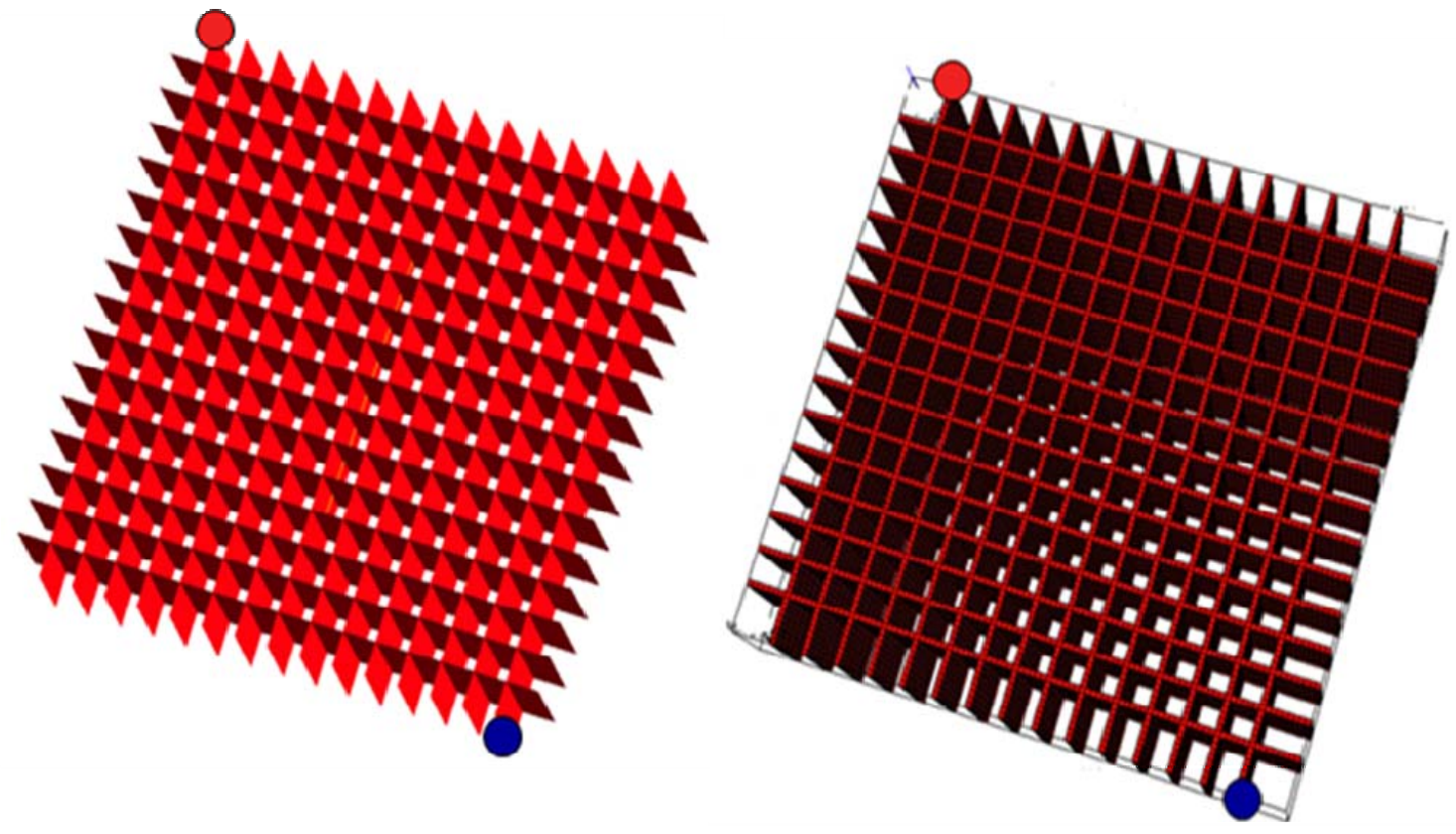

Figure 3.2 Regularized DFN basement model (left) and equivalent regularized ECLIPSE single porosity model

\section{Irregular (Hypothetical “Real”) DFN Basement Domain}

As shown in Figure 3.3, the properties in the irregularized DFN basement model were exactly the same as the regularized domain except for the feature orientation and connectivity. To distinguish the regular "ideal" basement model discussed in last section, this irregular domain was named the hypothetical "real" basement model. Two questions to be answered were: Can this hypothetical "real system" be easily represented by the equivalent "ideal system"? How could this domain be best represented by the traditional finite-difference based reservoir simulators such as ECLIPSE? 


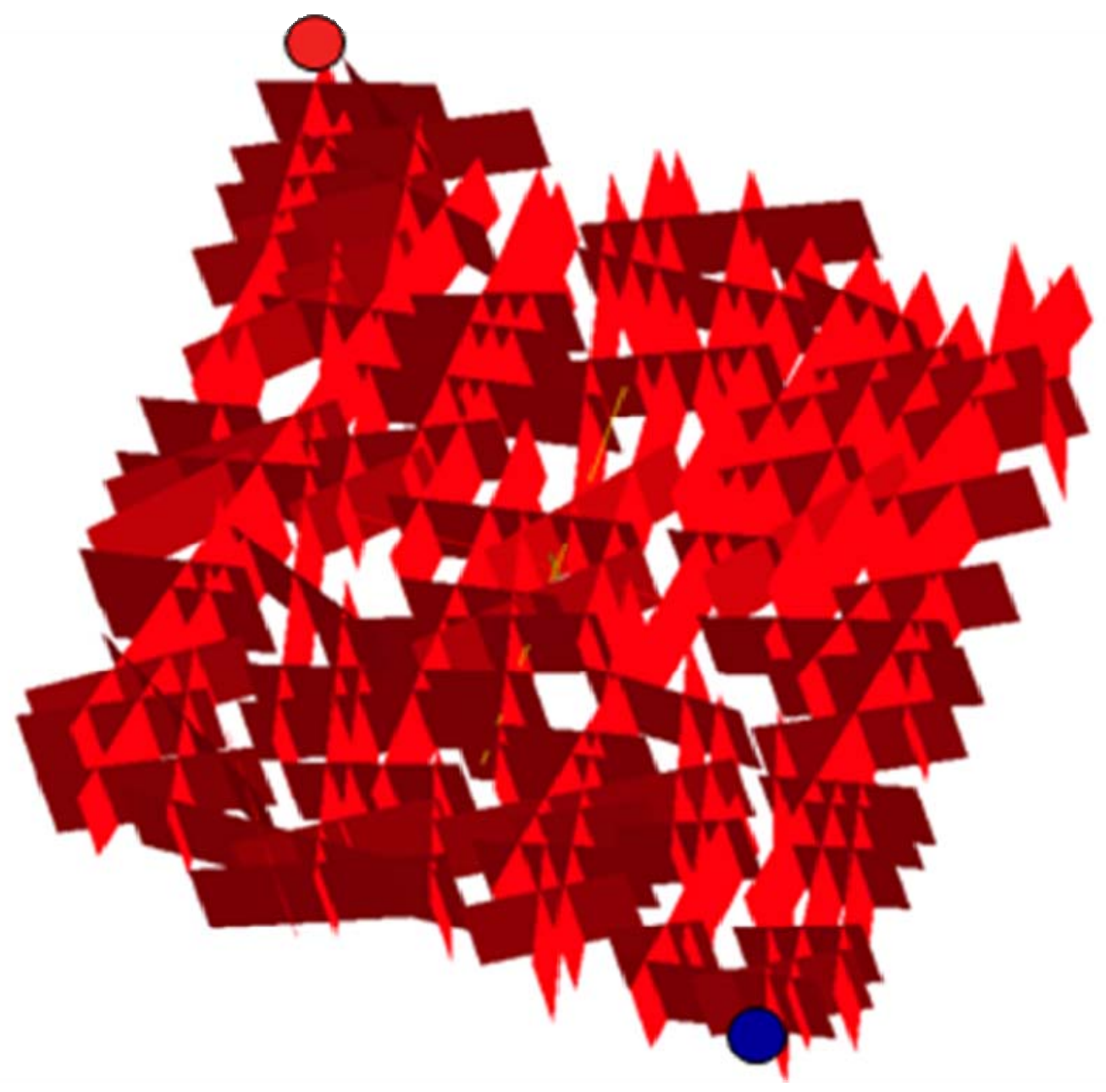

Figure 3.3 Irregularized DFN basement model with the same model properties as regularized DFN basement model but different orientation and connectivity

\section{“Real” System and Regular "Ideal” System}

Certain fracture network geological characterization analyses were done on both "ideal" and "real" systems. Figure 3.4 shows that the regularized "ideal" system has only two orientations as $(90,0)$ and $(180,0)$; for the hypothetical "real" system, the main fracture pole orientations are distributed from $(130,0)$ to $(350,0)$. Figure 3.5 shows that the regularized "ideal" system has the uniform feature equivalent length (radius) of 252 feet, but the fracture sizes of hypothetical "real" system offered power law fitting of $\mathrm{x} \_\min =252.1$ feet and exponential term $\mathrm{b}=5.2$. Detailed fracture network analysis is shown in Table 3.1. 
Fracture cluster analyses were performed on both "ideal" and "real" models. No isolated fracture was found in either system. The average fracture center was a little bit different:

1) Regularized "ideal system": $(500.00,500.00,100)$

2) Hypothetical "real system": $(513.06,488.70,100)$

\section{CVFE Simulator Verification}

The presence of fractures makes the reservoir domain geometrically complicated, particularly when the issue of connectivity dominates recovery. One of the key advantages of the DFN model, compared with conventionally fractured modeling, is to present the fracture orientation and connectivity explicitly. Obviously, the traditional finite-difference based simulator offered the option of the dual porosity model to simplify connectivity and heterogeneity issues that are modeled much more accurately by the DFN approach. The characterization by the DFN model requires rotatable permeability tensor along fractures/faults, which the finite-element discretization formula achieves especially well. A three-dimensional, three-phase black oil reservoir simulator was developed using a new CVFE formulation (Yang, 2003; Fu et al., 2005; $\mathrm{Fu}, 2007)$. In this new CVFE approach, flux-based upstream weighting was applied to ensure flux continuity both locally and globally. This weighting function solved the local mass conservation problem from traditional finite-element or CVFE simulations. 


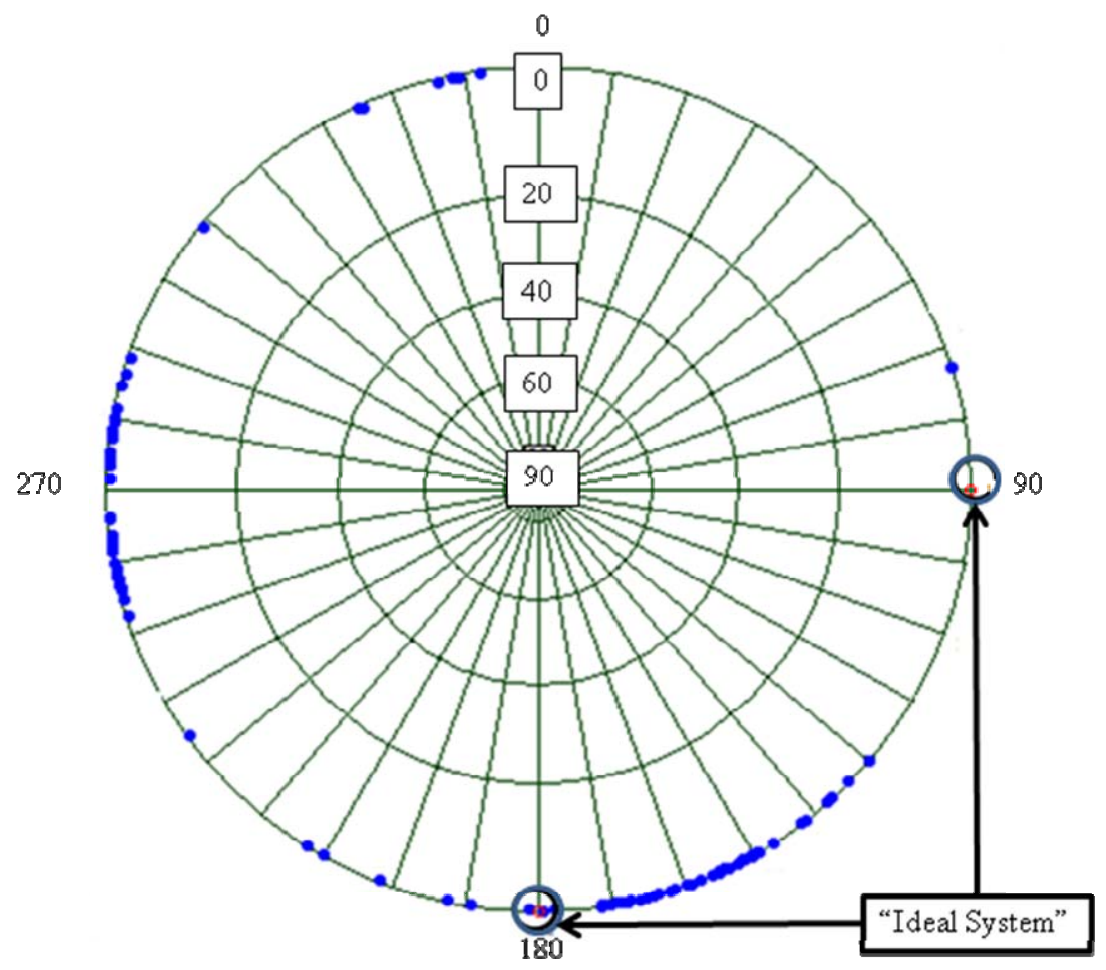

Figure 3.4 Fracture pole orientation (Wulff equal-angle projection, lower hemisphere) between hypothetical "real system" and "ideal system"

\section{Trace Data Power Law Plot}

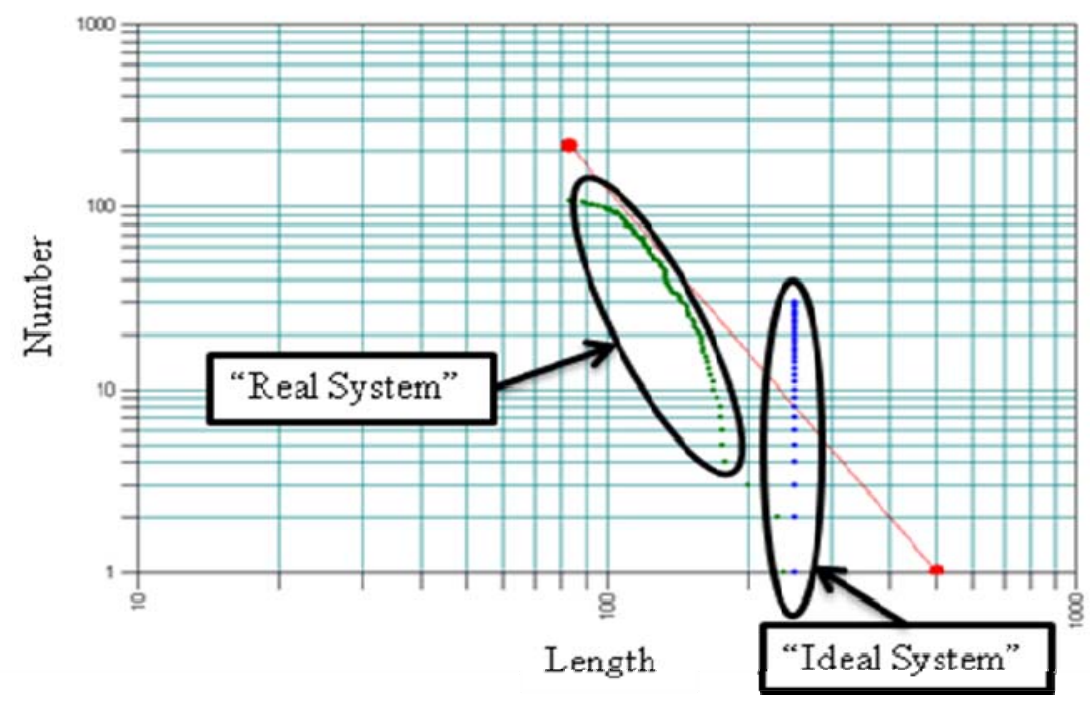

Figure 3.5 Comparisons of fracture size distribution between the "real" and "ideal" system 
Table 3.1 Fracture set statistics

\begin{tabular}{l|l|l}
\hline & Regularized "Ideal System" & Hypothetical "Real System" \\
\hline Number of fractures & 30 & 107 \\
\hline Total fracture area & 6000000 & 6000000 \\
\hline Total fracture volume & 3000000 & 3000000 \\
\hline P32 (fracArea/volume) & 0.02 & 0.02 \\
\hline P33 (fracVolume/volume) & 0.01 & 0.01 \\
\hline Mean orientation & 135,0 & $197.2836,0$ \\
\hline Equivalent radius mean & 252.13 & 130.59 \\
\hline Equivalent radius std dev & 1.06 & 28.34 \\
\hline Equivalent radius min & 252.13 & 83.40 \\
\hline Equivalent radius max & 252.13 & 237.99 \\
\hline Area mean & 200000 & 56075 \\
\hline Area std dev & 0 & 26292 \\
\hline Area min & 200000 & 21850 \\
\hline Area max & 200000 & 177946 \\
\hline Thickness, mean & 0.5 & 0.5 \\
\hline Thickness, std dev & 0 & 0 \\
\hline Thickness, min & 0.5 & 0.5 \\
\hline Thickness, max & 0.5 & 0.5 \\
\hline Permeability, mean & 1000 & 1000 \\
\hline Permeability, std dev & 0 & 0 \\
\hline Permeability, min & 1000 & 1000 \\
\hline Permeability, max & 1000 & 1000 \\
\hline Compressibility, mean & $5 \mathrm{e}-006$ & $5 \mathrm{e}-006$ \\
\hline Compressibility, std dev & 0 & 0 \\
\hline Compressibility, min & $5 \mathrm{e}-006$ & $5 \mathrm{e}-006$ \\
\hline Compressibility, max & $5 \mathrm{e}-006$ & $5 \mathrm{e}-006$ \\
\hline & &
\end{tabular}

Like all problems in the field of computation science and engineering, a new product needs to be "validated" and "verified" before it is actually implemented. The term validation means solving the right equation for a given problem and verification means solving the equations correctly. In the area of reservoir simulation, the same governing equations for a black-oil model have been widely used for the past few decades. Therefore, validation of a black-oil model is not considered for most new black-oil simulators. At the beginning, the focus of this study was to benchmark the CVFE simulator with the well-established commercial finite-difference based reservoir simulator ECLIPSE. This work could also be called as simulator verification by indexing method. 
On this verification work, the regularized "ideal system" was tested with 15 by 15 feature/faults strictly orthogonal in the domain of 1000 by 1000 by $200 \mathrm{ft}$. The matrix was considered to contribute zero porosity and permeability. The detailed reservoir properties are described in Chapter 3.4. This is a three-phase (oil, water and gas) study. Initially the reservoir pressure was over the bubble point which ensures all of the gas solute in the oil phase. One injection well and one production well were positioned at the diagonal direction of the reservoir domain as Figure 3.2 showed. Both wells horizontally penetrated the top of features. Bottom-hole pressure (BHP) controls were applied for well operation. Since the DFN approach presents feature/faults as a two-dimensional plane in the three-dimensional space, the domain was meshed into triangles which shared the same side or node at the region of feature/faults interaction. For ECLIPSE simulator, the features were presented by the stack of grid blocks which have the actual width of the features $(0.5 \mathrm{ft})$ or equivalent thickness by the constraint of constant $k h$ value (500 md-ft). To speed up calculations, the matrix blocks other than features in the ECLIPSE were set as nonactive. Another constraint for both the "ideal" and "real" systems was to set the constant total original oil in place (OOIP) as 53580 STB. The discretized domain for the DFN approach and conventional finite-difference are shown in Figure 3.6 and Figure 3.7.

Figure 3.6 and Figure 3.7 show that both the DFN triangular mesh and the ECLIPSE grid blocks have five element/grid layers in the z-direction. The simulations were run for 900 days with the first 300 days as the primary production period. Starting from day 301, simulation was considered to be secondary recovery with the water injector being introduced. 

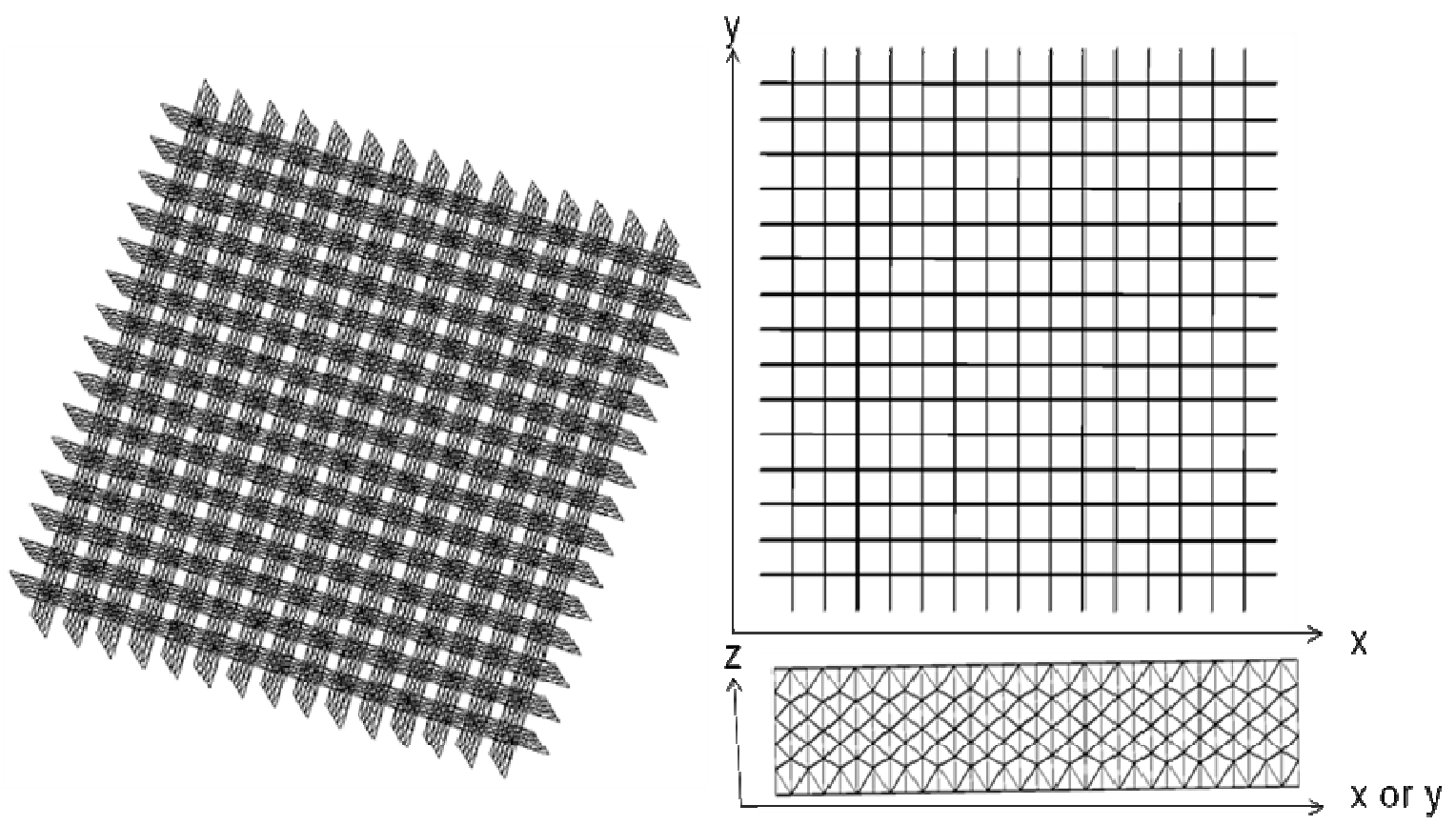

Figure 3.6 Three-dimensional view (left) and two-dimensional view (right) of discretized regularized, DFN modeled "ideal" basement reservoir domain for the CVFE simulator
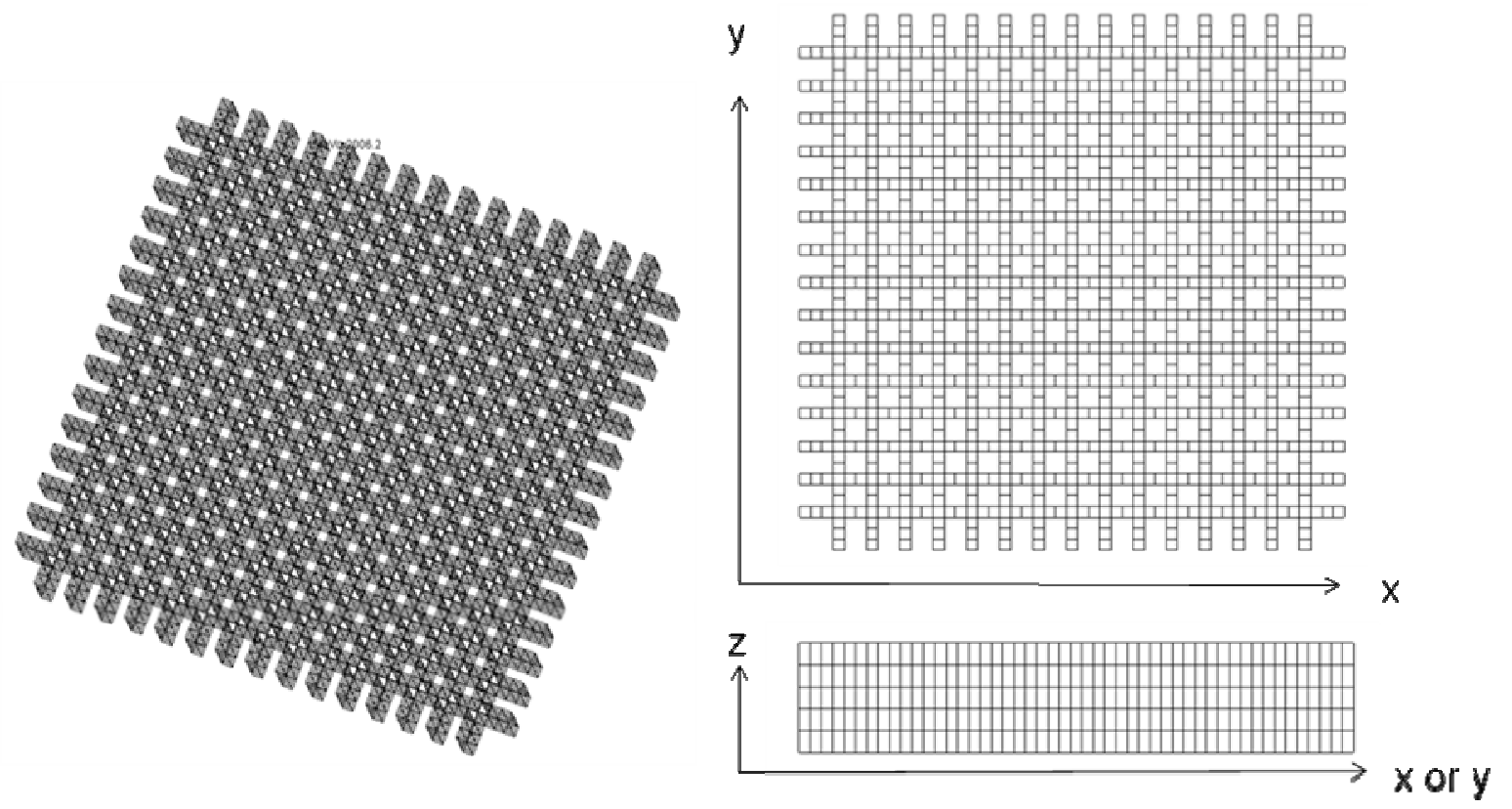

Figure 3.7 Three-dimensional view (left) and two-dimensional view (right) of discretized, regularized finite-difference modeled "ideal" basement reservoir domain for the ECLIPSE simulator 
Simulation results from CVFE and ECLIPSE simulators such as oil production rates and oil recovery are compared in Figures 3.8 and 3.9. Figure 3.8 and Figure 3.9 show that results of both oil production rate and oil recovery from CVFE simulation are almost the same as the results from ECLIPSE. Since the regularized basement model is strictly orthogonal (which can be accurately calculated by ECLIPSE), this close agreement between results made a very positive conclusion about the CVFE simulator. Note that this case study was performed under the threephase condition, and with the solution gas involved, the simulation results showed that CVFE simulator was benchmarked with ECLIPSE black-oil simulator remarkably well. This conclusion can also be observed from oil distribution snapshots shown in Figure 3.10.

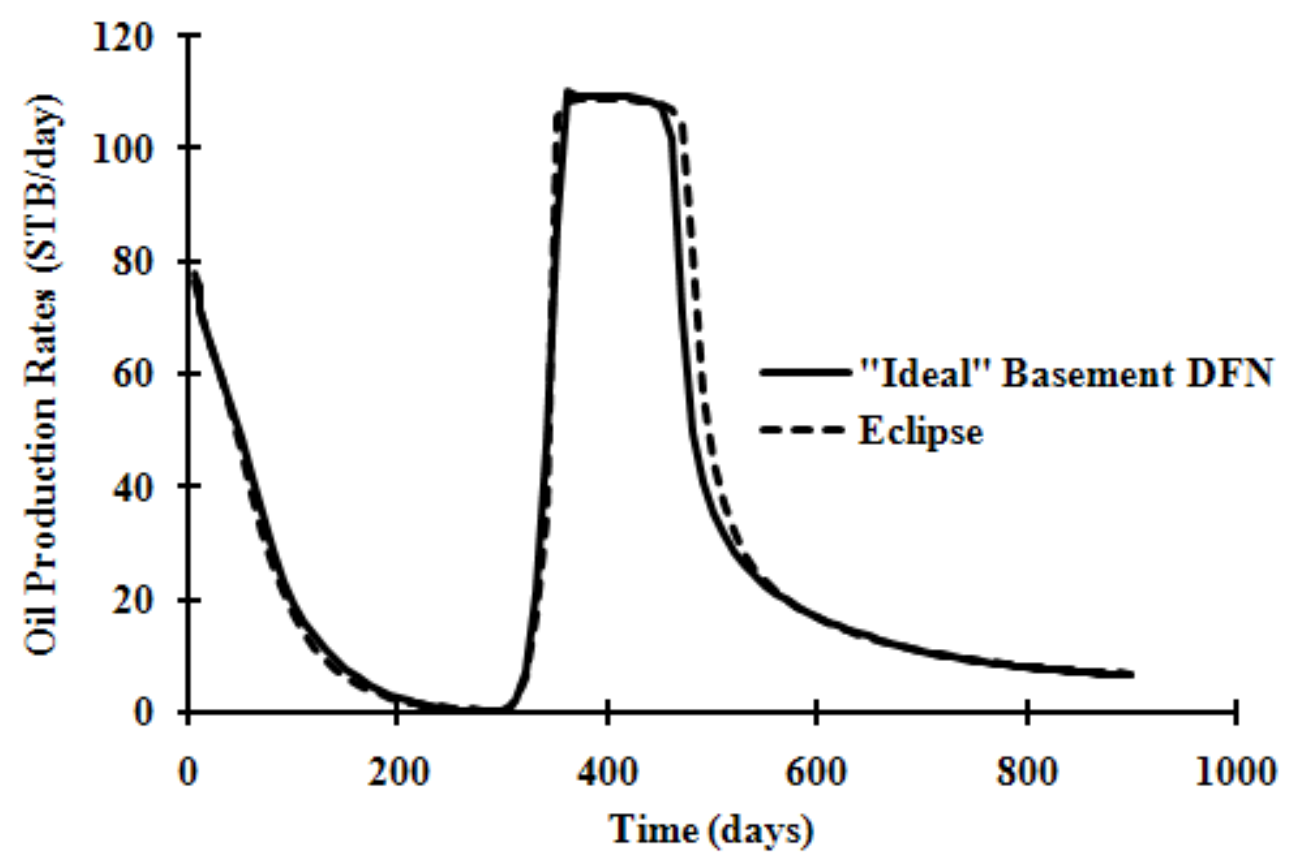

Figure 3.8 Comparisons of oil production rate between the DFN modeled CVFE simulator and the single porosity modeled ECLIPSE simulator for the regularized "ideal system" 


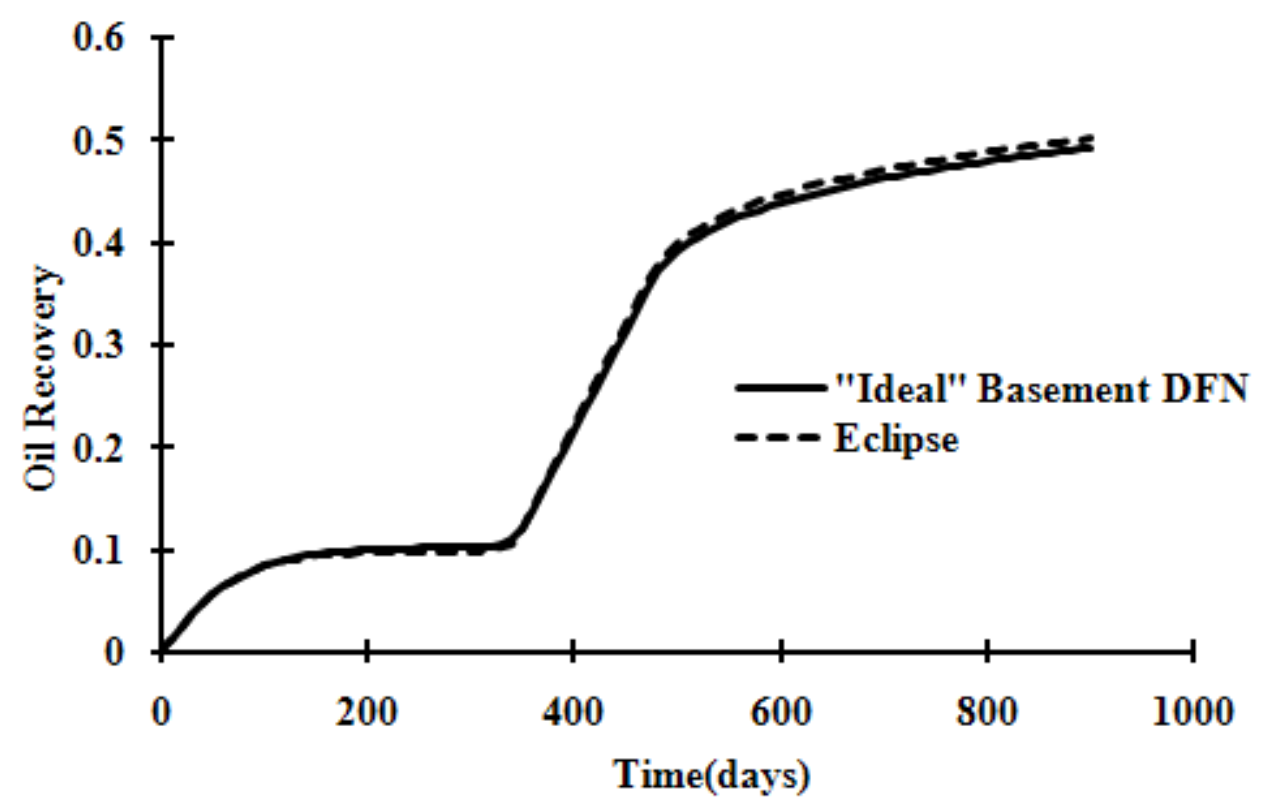

Figure 3.9 Comparisons of oil recovery between the DFN modeled CVFE simulator and the single porosity modeled ECLIPSE simulator for the regularized "ideal system" 

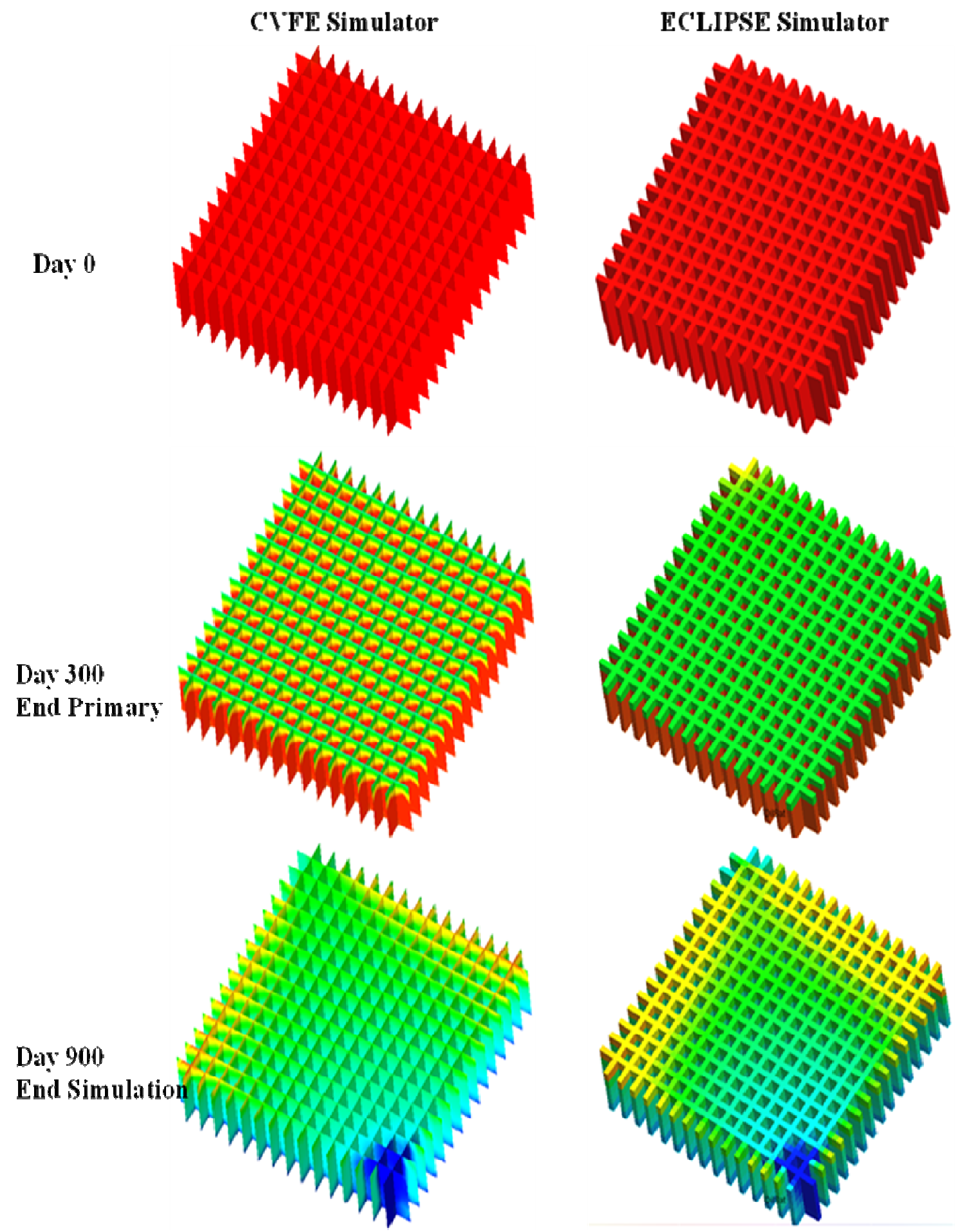
The verification of constraints (constant kh value and constant OOIP) are shown in Figure 3.11. At day 900 (end of simulation), simulations with $0.5 \mathrm{ft}$ to $20 \mathrm{ft}$ fracture thickness had reached the same the residual oil distributions from the ECLIPSE simulator.

The indexing verification methodology adopted by this study was commonly applied to verify a new reservoir simulator in the petroleum industry. Other more strict mathematical verification work has been done (Yang, 2003; Fu, 2007) on the CVFE formulation by implementing the manufactured solution method. In the manufactured solution approach, a solution function that satisfied both initial and boundary conditions was first synthesized or manufactured. That solution function was then substituted into the governing equation to find the corresponding source function. Mathematically, the solution obtained from the CVFE simulator showed an excellent order of convergence rate with the manufactured solution.

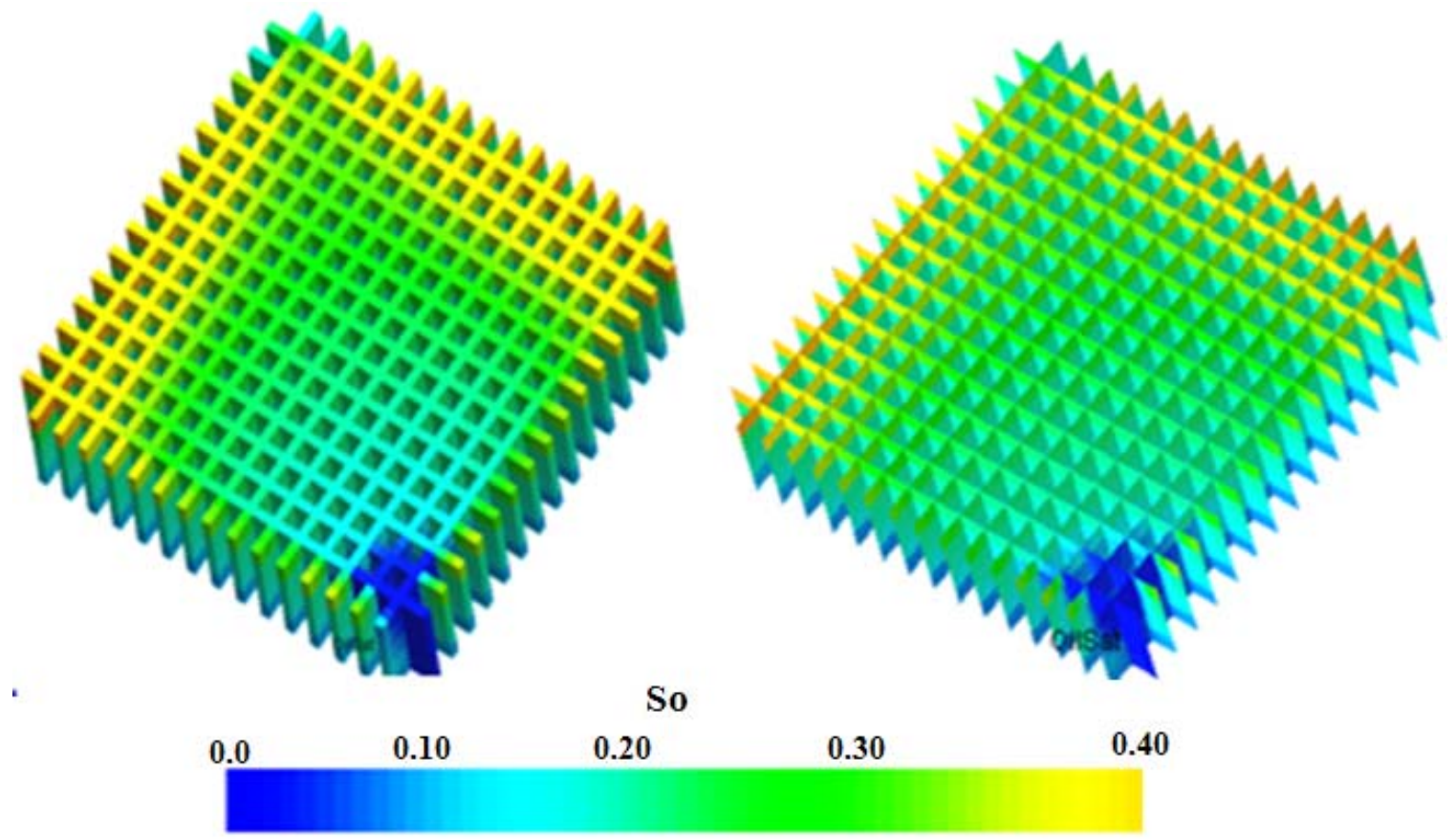

Figure 3.11 Comparisons between feature/fault grids block thickness (left 20ft; right $0.5 \mathrm{ft}$ ) by applying two constraints: OOIP \& kh value. 
Through this indexing verification with the well-known ECLIPSE simulator, now the accuracy of the CVFE simulator is ascertained. Some sensitivities of DFN characteristics are discussed below.

\section{Applications}

Uncertainty exists around the fractured reservoir due to the fracture network's heterogeneous properties. These fracture network properties include connectivity, permeability distribution, dip orientation, and size distribution. To demonstrate the impact effects of uncertain elements on the multiphase oil recovery scheme, as shown in Figure 3.12, this study used a "real" fracture network generated by a network of intersecting faults that mimic a more realistic basement reservoir.

\section{Permeability Variations Studies}

Permeability is an important property of the porous medium and is a measure of the capacity of the medium to transmit fluids. Hydrocarbon reservoirs can have primary and secondary permeability.

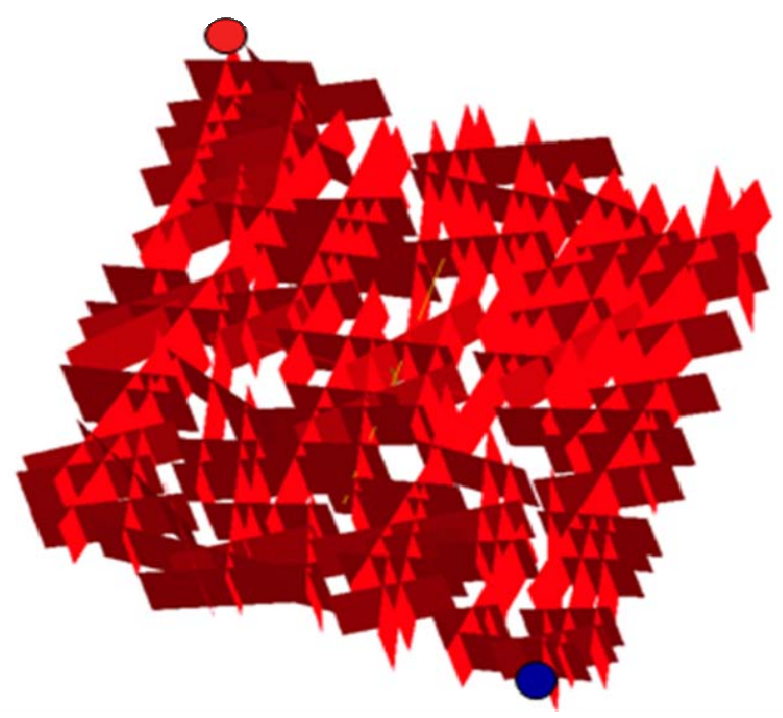

Figure 3.12 Irregularized DFN basement model with the same model properties as regularized DFN basement model with different orientation and connectivity 
Primary permeability is referred to as matrix permeability by reservoir engineers. Secondary permeability can be by either fractures or solution vugs. In this chapter, only the Type I (basement type) reservoirs are considered. Therefore, all the permeability discussed in this chapter is fracture permeability. Generally fracture permeability abides by cubit law of fracture thickness. Since the fracture is generated due to the stress and strain acting on the rock, the thickness of fractures cannot be homogeneous. This results in a highly heterogeneous permeability distribution within each fracture/fault. In this section, the hydrocarbon recovery scheme from three homogenous permeability models (1000 md, $100 \mathrm{md}, 10 \mathrm{md})$ and two heterogeneous permeability models (with random geometric mean of $100 \mathrm{md}$ ) have been compared in order to demonstrate the fracture network uncertainty.

The homogenous permeability models are easily understood. The results of oil saturation distribution snapshots from various homogeneous permeability models are shown in Figure 3.13.
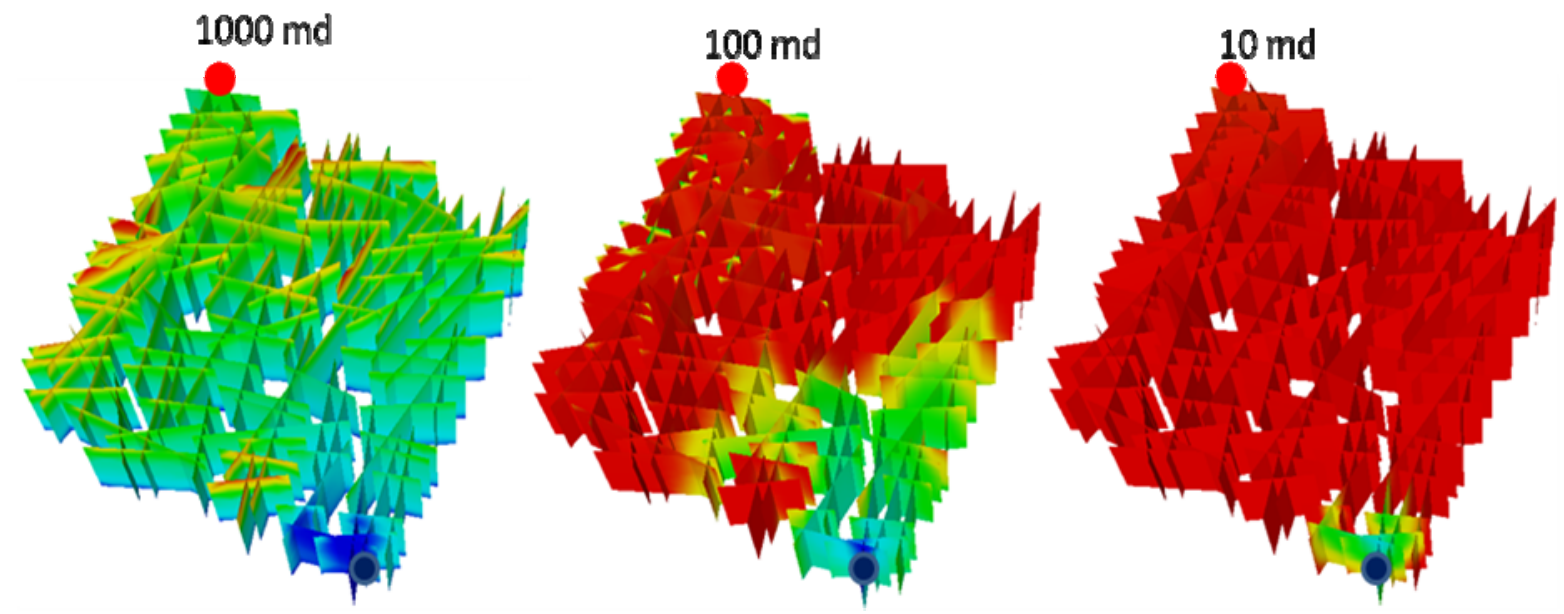

Figure 3.13 At day 900, oil saturation distribution snapshots from different homogenous permeability models

Figure 3.14 demonstrates that with lowering the homogeneous permeability by one or two magnitudes in the whole basement reservoir, water breakthrough times were delayed 
significantly. The simulated water breakthrough time, after the water injections, is 185 (1000 $\mathrm{md}), 3200(100 \mathrm{md})$, and $14700(10 \mathrm{md})$. The calculation geometric mean of a permeability data set $\left[\mathrm{k}_{1}, \mathrm{k}_{2}, \ldots, \mathrm{k}_{\mathrm{n}}\right]$ could be described by the Equation (3-7):

$\left(\prod_{i=1}^{\mathrm{a}} \mathrm{k}_{1}\right)^{1 / n}=\sqrt[n]{\mathrm{k}_{1} \cdot \mathrm{k}_{2} \cdots \cdots \cdot \mathrm{k}_{\mathrm{n}}}$

where $\mathrm{k}$ is the permeability, and $\mathrm{n}$ represents the numbers of discretized elements.

Mathematically, geometric mean is an average indicating the central tendency or typical value of a set of number. It can be understood in terms of geometry. The geometric mean of two numbers, $a$ and $b$, is simply the side length of the square whose area is equal to that of a rectangle with side lengths $a$ and $b$. that is what is $\mathrm{n}$ such that $n^{2}=a x b$. Similarly, the geometric mean of three numbers, $a, b$, and $c$, is the side length of a cube whose volume is the same as that of a rectangular prism with side lengths equal to the three given numbers. This geometric interpretation of the mean is probably what gave it its name. It is important to note that the geometric mean applies only to positive values to prevent the result of imaginary numbers. For example, no negative fracture permeability exists physically. However, physically there might be some "zero" permeability existing inside the fault. In that case, other average methods might be applied instead of the geometric mean. 


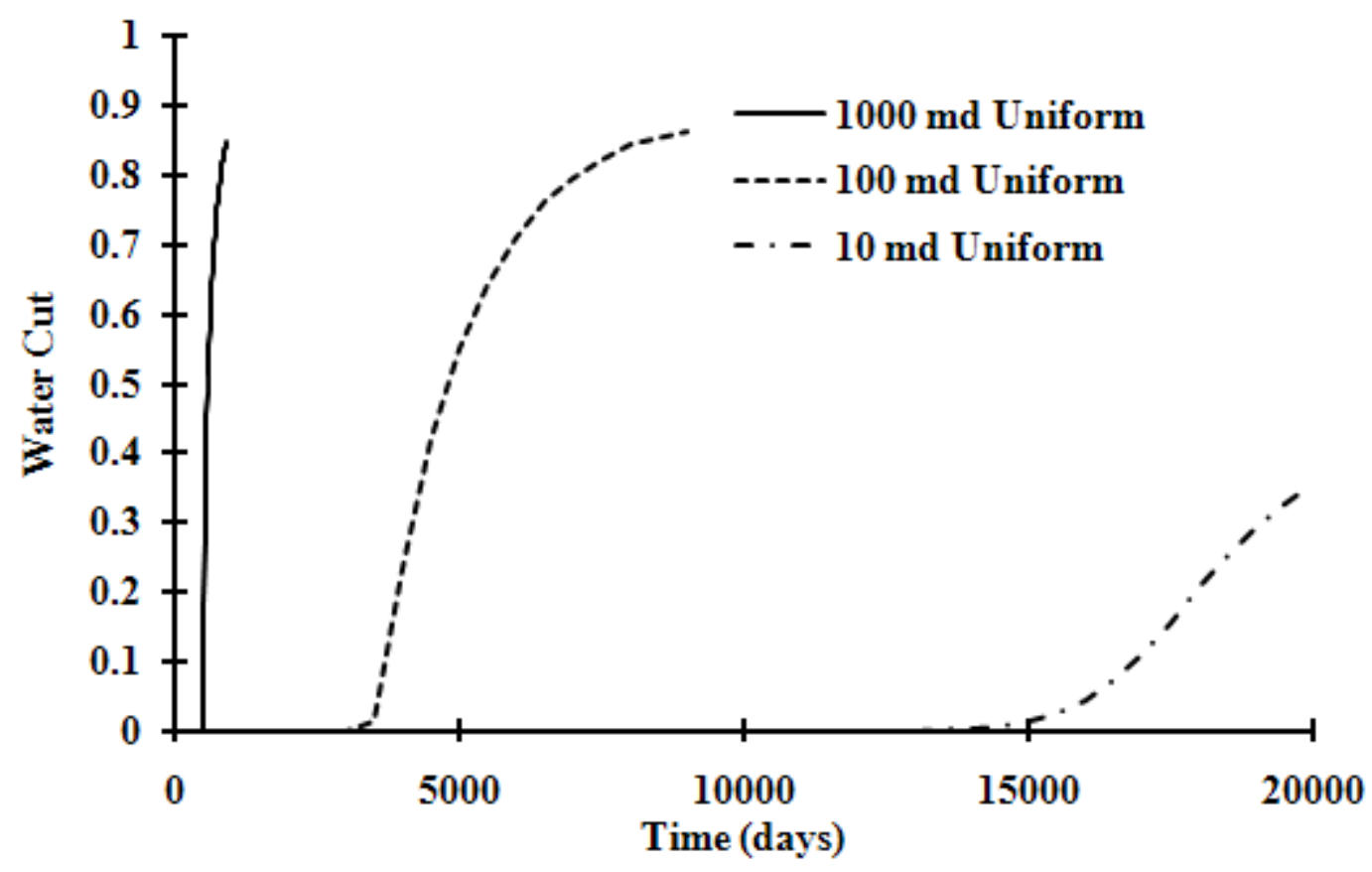

Figure 3.14 Comparisons of water breakthrough and water cut among different homogeneous permeability models

Figure 3.15 illustrates the geometric mean based intrafault permeability distributions. If the intrafault were composed of two types of permeability values: 10 and values. Then the permeability of discretized elements can be characterized based on the percentage distribution. If the intrafault were composed of three types of permeability $(10,100$ and $1000 \mathrm{md})$, the geometric mean of 100 md will result in one-third quantity distributions with each permeability value. 


\section{$50: 50(10,1000 \mathrm{md})$ Geom. Mean $=100 \mathrm{md}$}

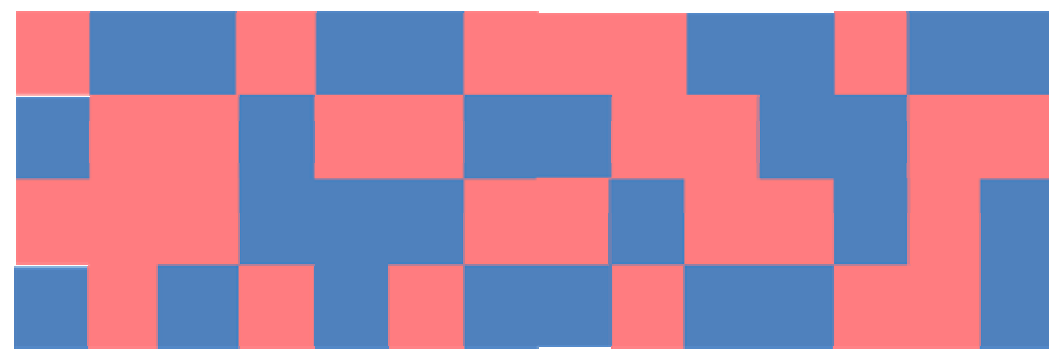

\section{$k$ (md)}

1000

100

$33: 33: 33(10,100,1000 \mathrm{md})$ Geom. Mean $=100 \mathrm{md}$

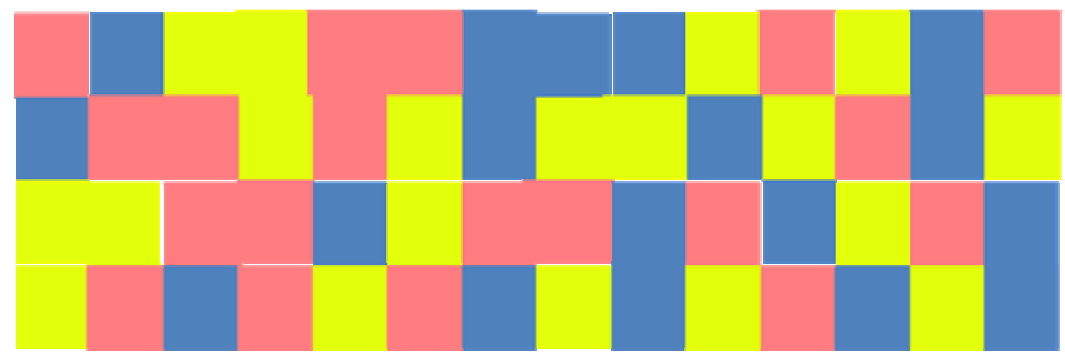

Figure 3.15 Illustrations of geometric mean based intrafault permeability distributions $1000 \mathrm{md}$, the $100 \mathrm{md}$ geometric mean will give the half-half percentage of the two permeability

Figure 3.16 shows residual oil saturation of three basement fracture networks (one uniform 100 md case and two various 100md geometric mean domains). As previously shown in the simulation, all three cases passed primary production stage (first 300 days) and secondary production (from day 301 to 900). Obviously, the water flooding patterns are totally different by different fracture network characterizations: uniform 100md case study offers the medium performance between the two types of $100 \mathrm{md}$ geometric mean permeability values, the one-third permeability distribution cases result in the best water flooding and the half-half case offers the worst water flooding scenario. 

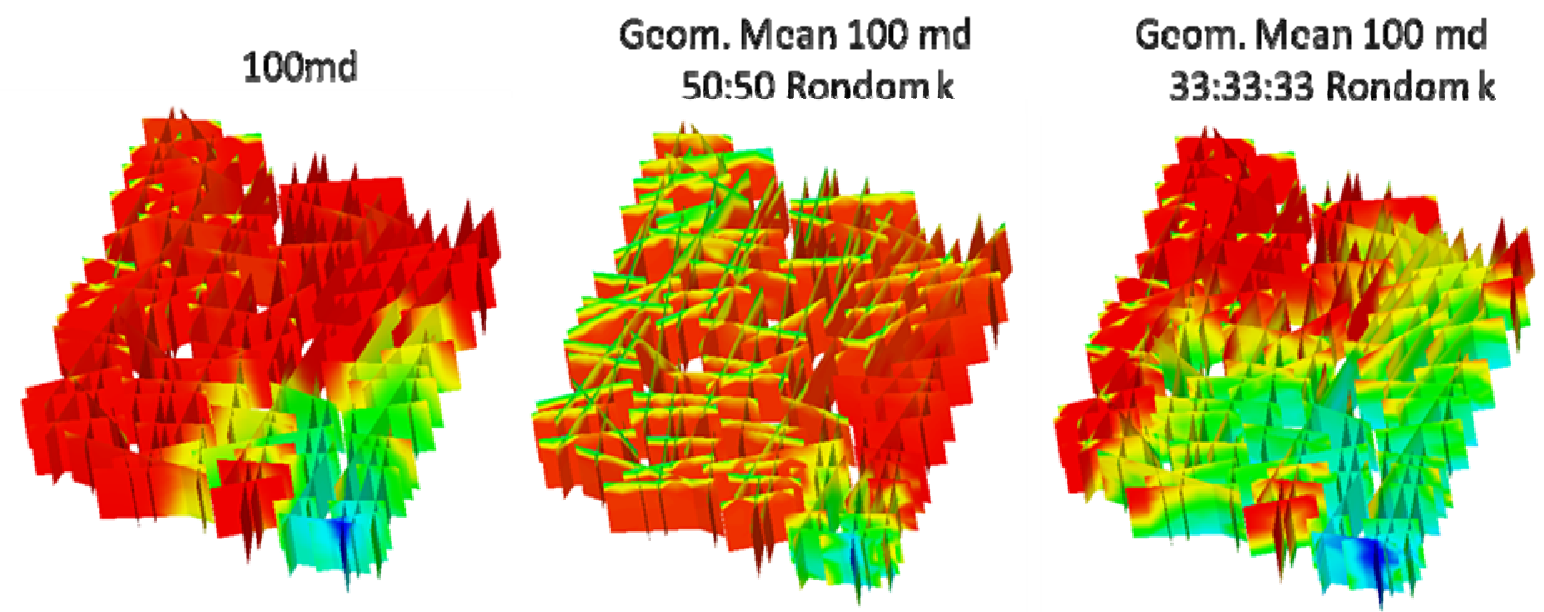

Figure 3.16 At day 900, oil saturation distribution snapshots from different homogenous permeability models

Figure 3.17 to 3.19 compared simulation results as the oil production rate, oil recovery and water cut. Obviously, the randomized intrafault permeability distributions brought totally different hydrocarbon recovery mechanisms to our proposed basement domain. The figures of the oil production rate, water breakthrough time, and water cut scales show possible large-range variations. In the random permeability distribution case, the oil production rate could be higher than in the uniform permeability case, due to possible high permeability channels (with those 1000 md characterized elements); the oil production rate also could be lower than in the uniform permeability case due to the low permeability in the main water flooding channel (with $10 \mathrm{md}$ characterized elements). However, if a certain number of simulations were performed based on different random seeds, some uncertainty range might be identified. The more simulations performed, the more accurately the risk range might be described. Based on this hypothesis, a set of geometric mean $(33: 33: 33) 100$ md simulations have been performed. The only difference among these simulations is the detailed element permeability distributions on each single feature. The water cut results of these simulations are shown in Figure 3.20. 


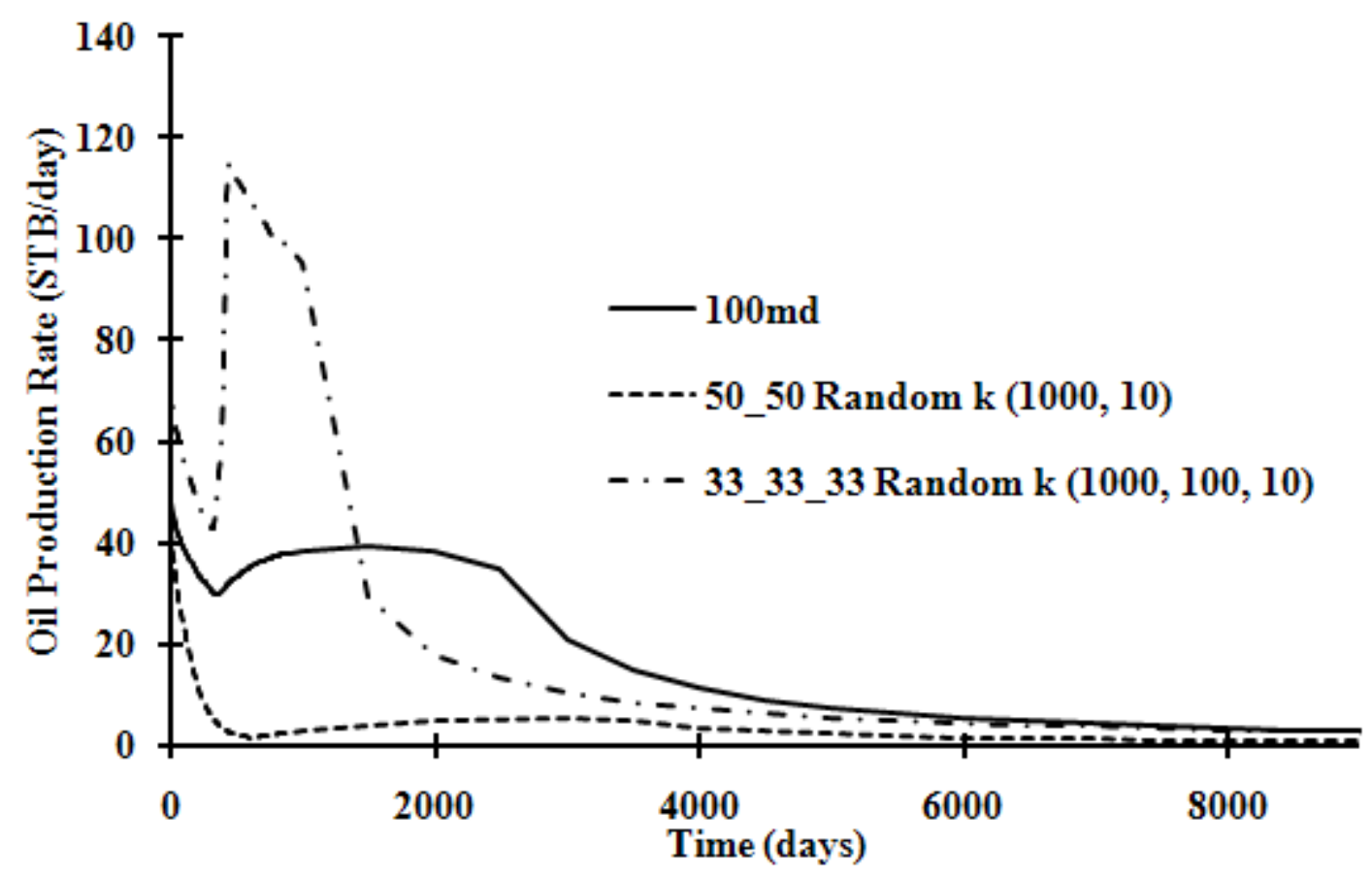

Figure 3.17 Oil production rate comparisons among uniform $100 \mathrm{md}$ and geometric mean 100 md cases

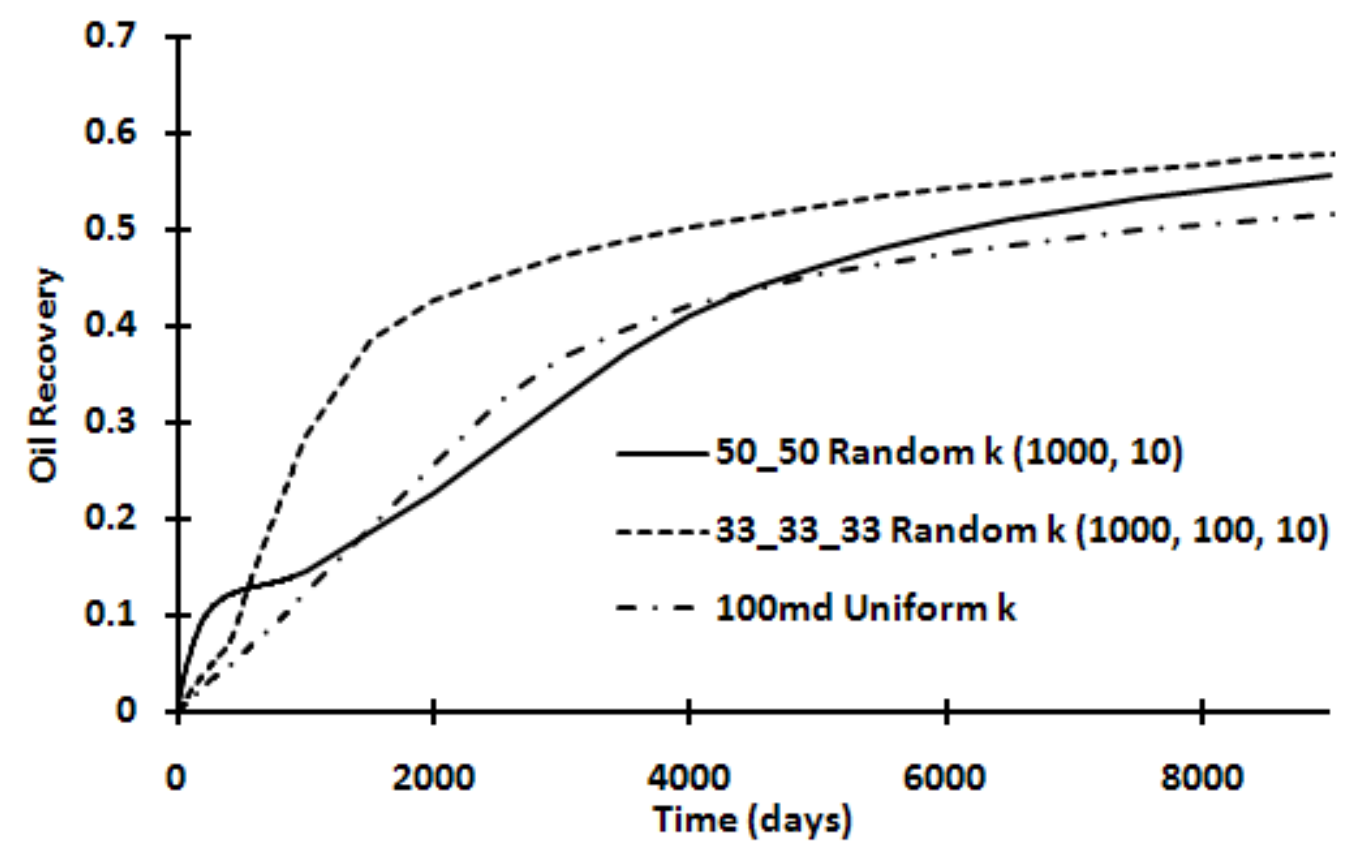

Figure 3.18 Cumulative oil production comparisons among uniform 100 md and geometric mean 100 md cases 


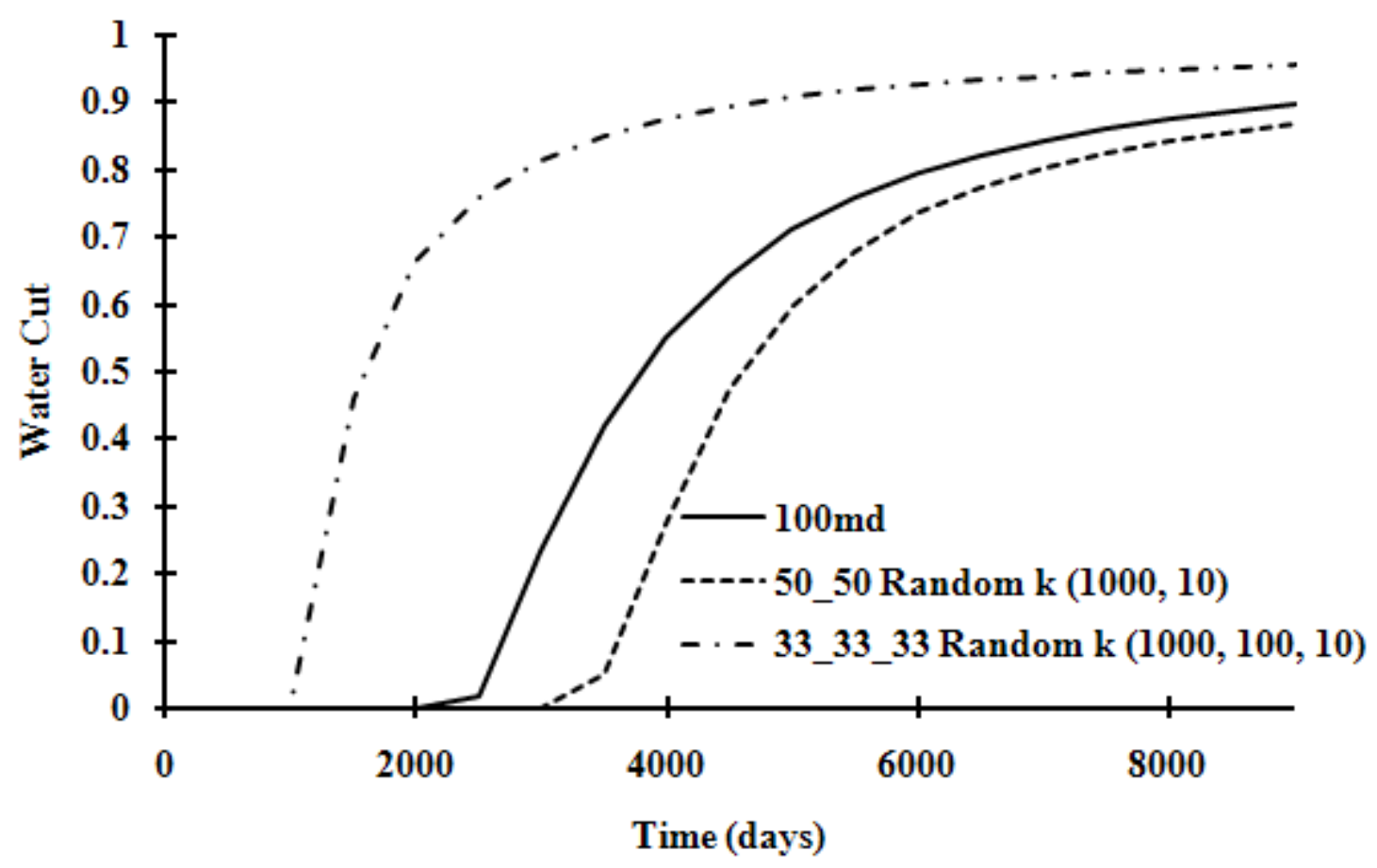

Figure 3.19 Water cut comparisons among uniform $100 \mathrm{md}$ and geometric mean $100 \mathrm{md}$ cases In the water flood case studies, water cut and water breakthrough time are the principal ways of making reservoir management decisions. Figure 3.20 shows a large range of water breakthrough times. For the same domain with the exact same operating conditions, the water flooding patterns were totally different. This type of modeling mimics big impacts on the pattern of sweep, due to the uncertainty of the fracture/fault permeability distributions. In a way, this is important for understanding the fracture network uncertainty when attempting to develop innovative approaches to reservoir management. 


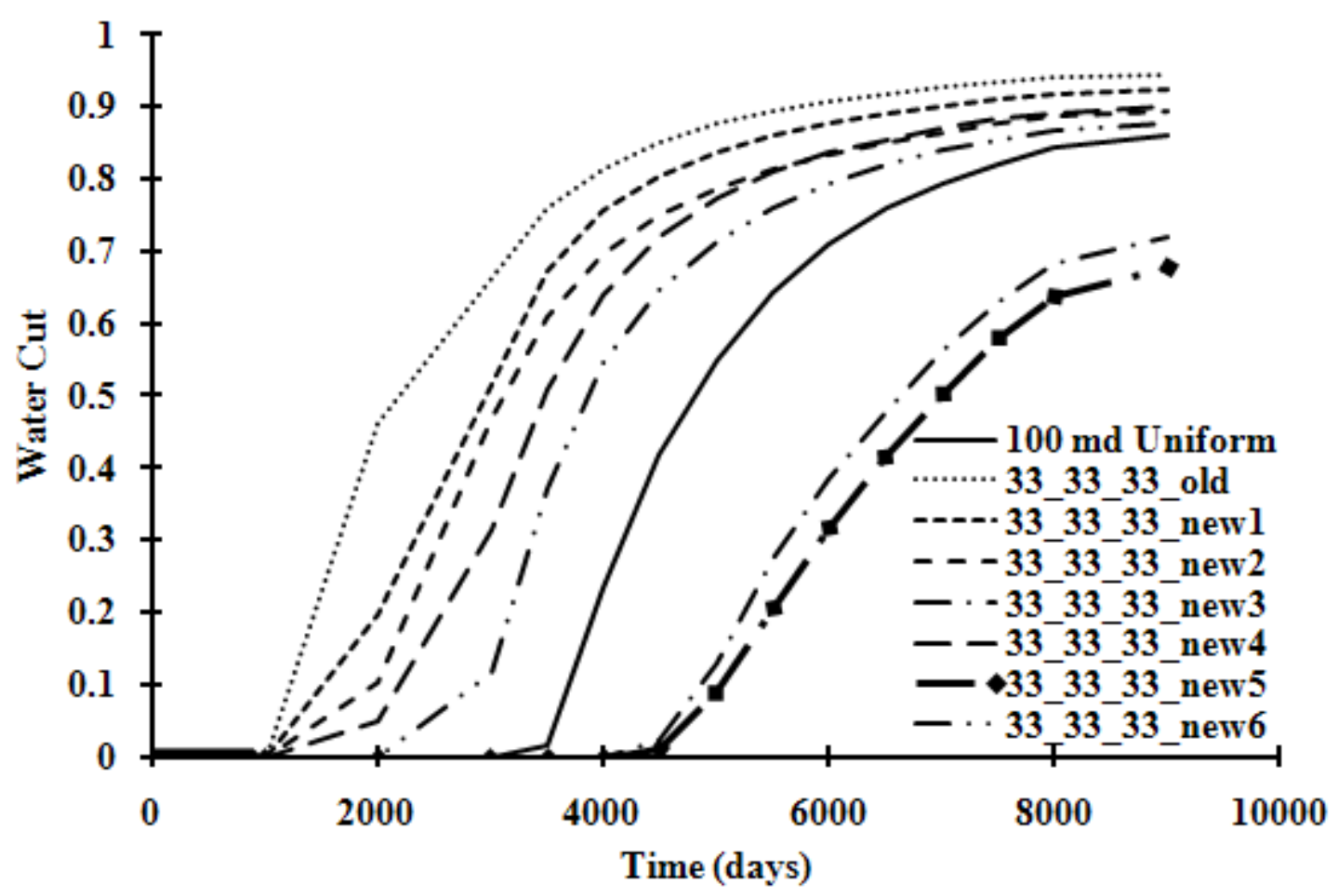

Figure 3.20 Water cut comparisons for 33_33_33 geometric mean with random seeds

Some conclusions can be drawn from the studies in this section:

1) The rock permeability is an important parameter of fluid flows inside the fracture networks. The variation of rock permeability distributions results in a huge uncertainty, particularly for the secondary production with water flooding.

2) Introducing spatial variability in permeability within the fault planes (using spatially randomized patterns of 10,100 and $1000 \mathrm{md}$ ), while retaining a constant geometric mean permeability of $100 \mathrm{md}$, yields enhanced oil production due to the highpermeability pathways. And these kind of pathways result in different oil recovery factors for the long-term or short-term view, which might suggest totally different recovery plans for reservoir management. 


\section{Fracture Network Connectivity Studies}

The connectivity of fracture/fault in subsurface rock formations is a key factor in understanding and predicting fluid flow in hydrocarbon reservoirs. This is especially true in Type I basement reservoirs, since in this type of reservoir all hydrocarbons exist inside the fractures, and the fractures provide essential permeability. This permeability then makes the connectivity problem fatal for the hydrocarbon production. Some impacts, due to the connectivity, were already shown in the simulations in the above two sections.

An example of residual oil resulting from the fracture network's connectivity is shown in Figure 3.21. In this figure, some oil traps (circled) result from the fracture connectivity. Obviously these main traps are limited in connection with the major water flooding way between the injector and the producer.

In this section, the critical fracture/fault connections between the injector and the producer are identified by the trace map in Figure 3.22. Figure 3.22 demonstrates that the fracture networks were divided into two separate zones: west zone (left-side) and east zone (right-side). These two zones are connected by four east-west fractures/faults. The injector and the producer are located in different zones. The whole reservoir is assigned with uniform permeability at $1000 \mathrm{md}$. 


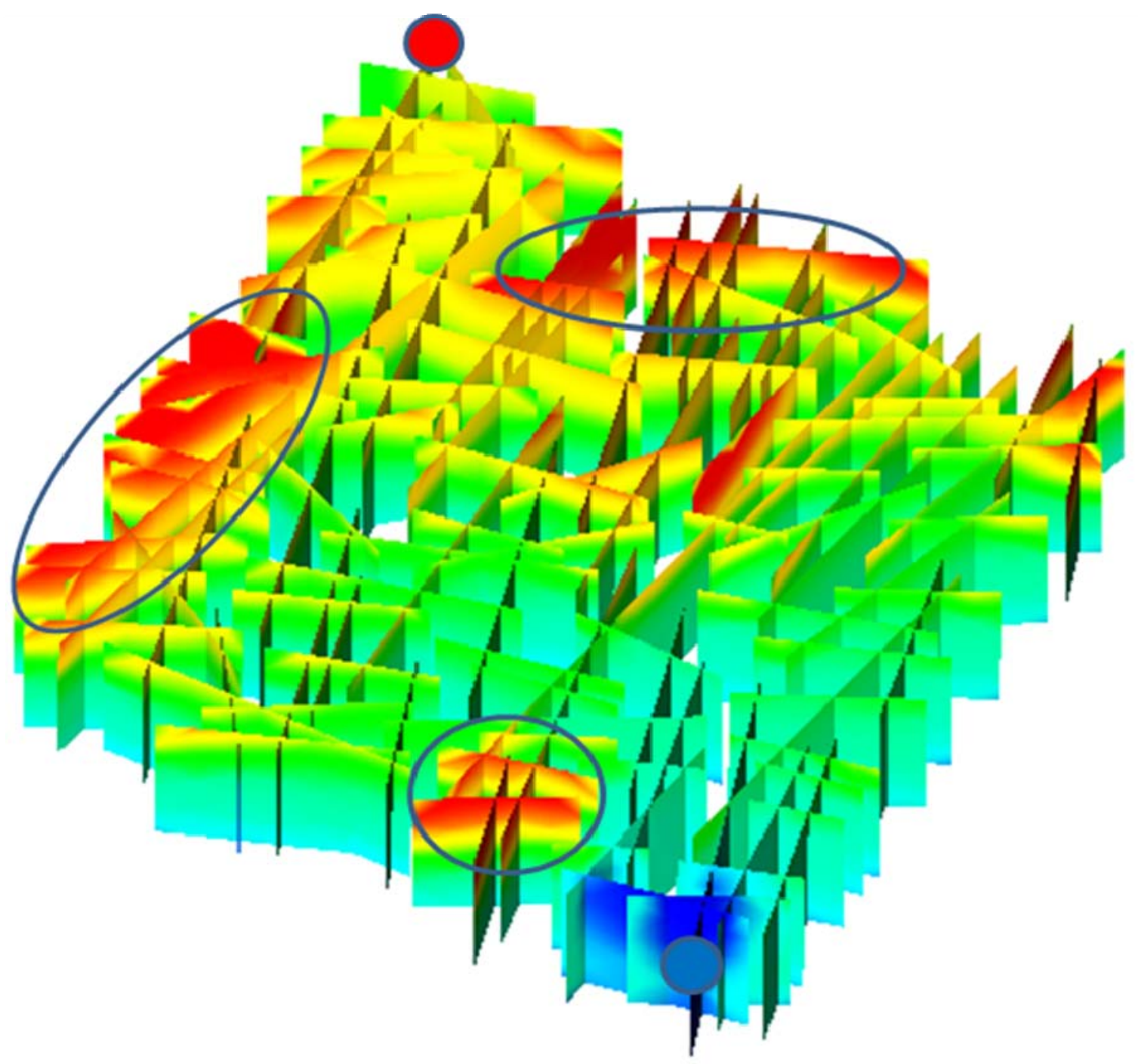

Figure 3.21 Residual oil saturation distribution snapshot due to the fracture network connectivity (Case 1000 md uniform $\mathrm{k}$ with day 600) 


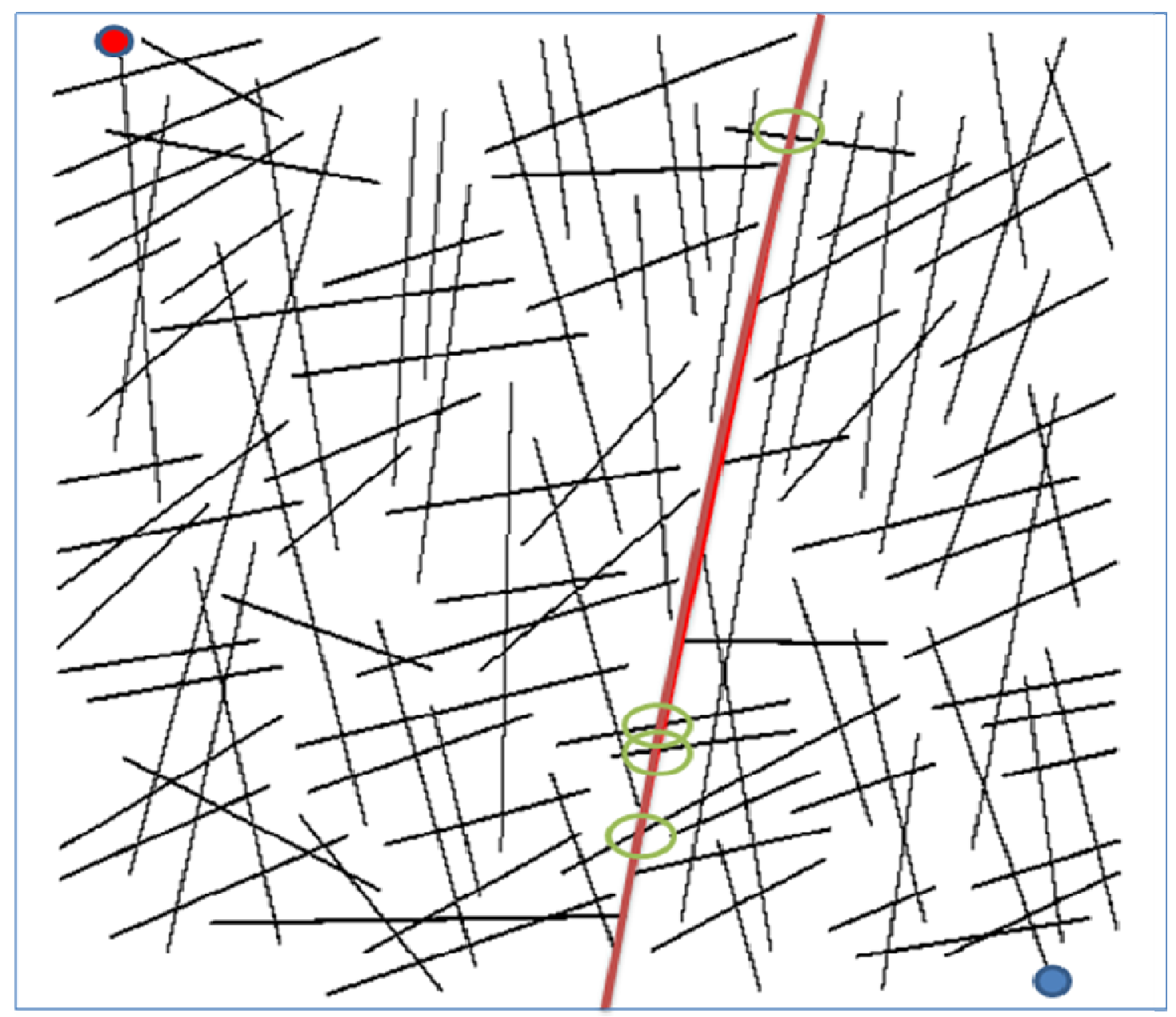

Figure 3.22 Illustration of two separate fracture zones (separated by the acclivitous line in the middle of trace map) connected by four fractures (circles)

The hypothesis for studying the fracture connectivity and how it impacts oil production was produced by comparing the productions from the original fracture network and the disconnected fractured network (disconnecting the bottom three connections).

Figure 3.23 presents the large difference in oil saturation distribution at the end of the simulation: obviously, since three main connections on the south were disconnected, the only bridge between the injector cluster and the producer cluster was on the north side of the fracture network. There are large amounts of oil on the south-west corner of the proposed basement domain that cannot be swept out. These differences are due to the connectivity of the fracture network. 

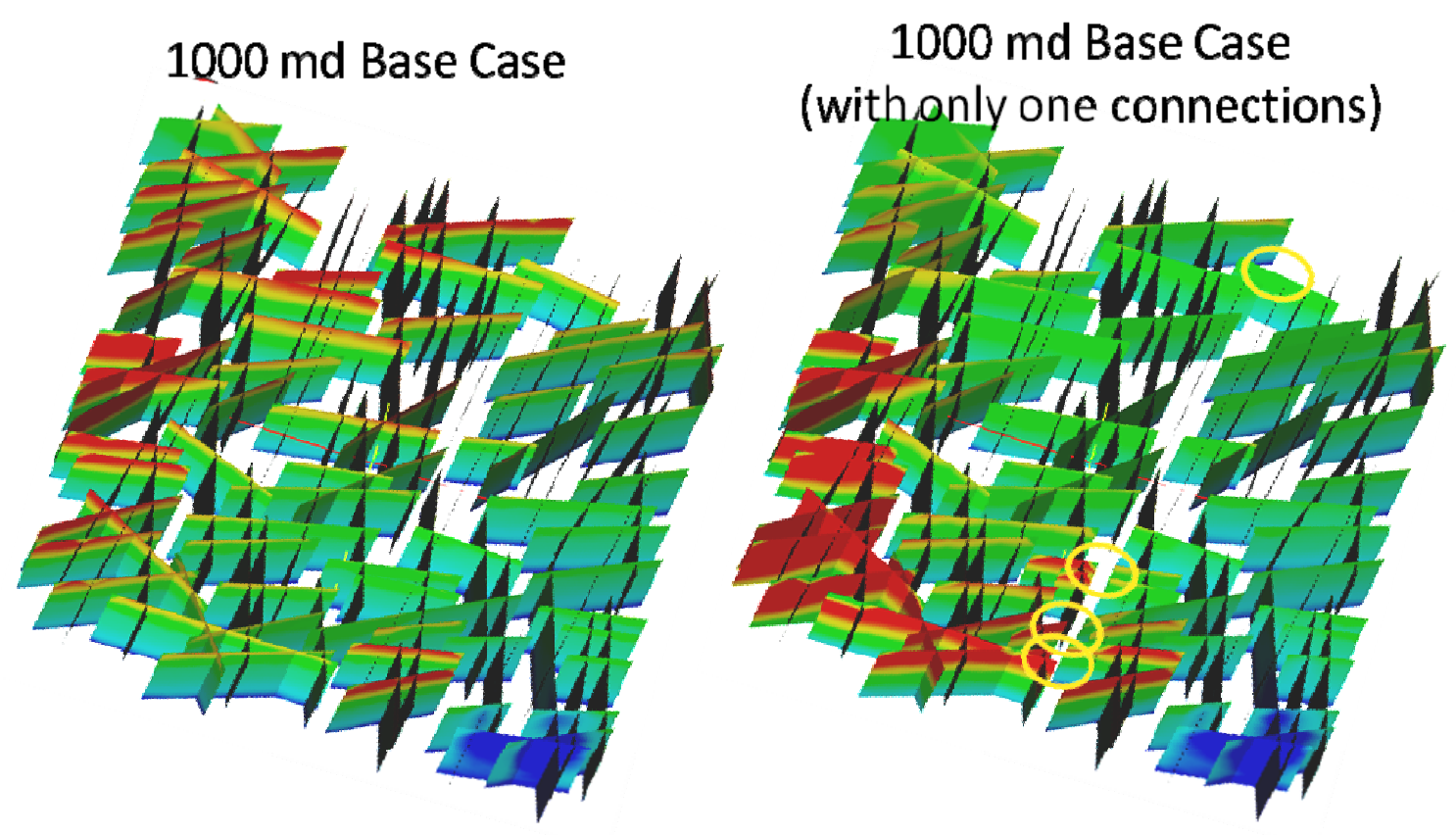

Figure 3.23 Comparisons of oil saturation distributions between the original fracture network and disconnected model at day 900

To a large extent, the fracture network connectivity does not impact the primary productions. During the primary production stage, solution gas provides the main reservoir driving force. Since the gas mobility is large, and as long as the whole fracture network is connected (even if it has only one connection), the oil production rates from both simulations are almost identical. However, on the secondary production stage, the fracture network connectivity plays an important role.

Figure 3.24 shows that the peak oil production rate was reduced about $65.1 \%$ by disconnecting the lower three connections. Figure 3.25 shows that there is $12.6 \%$ cumulative oil production reduction on the disconnected model. 


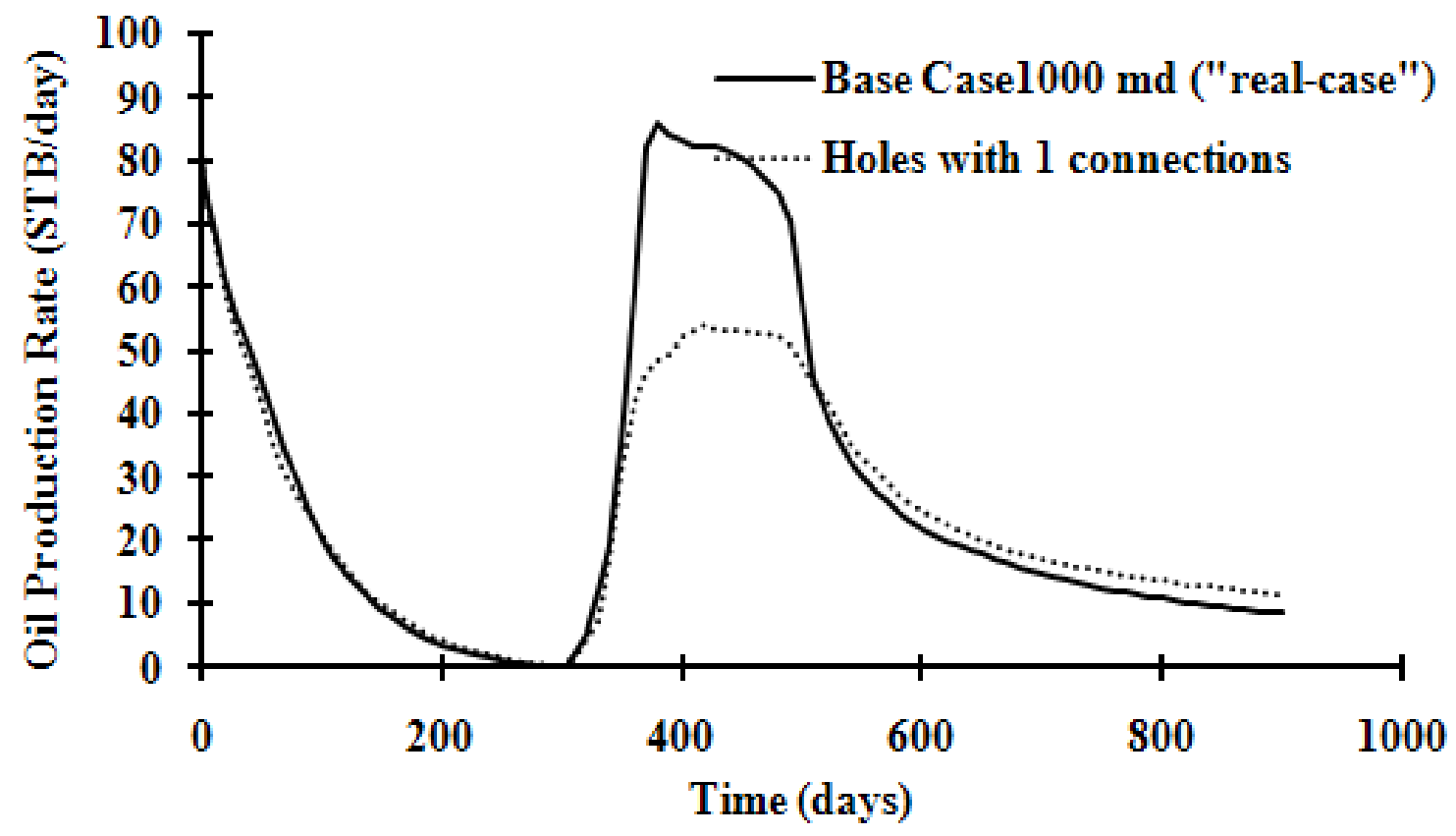

Figure 3.24 Oil production rate comparisons between base case and reduced connections case

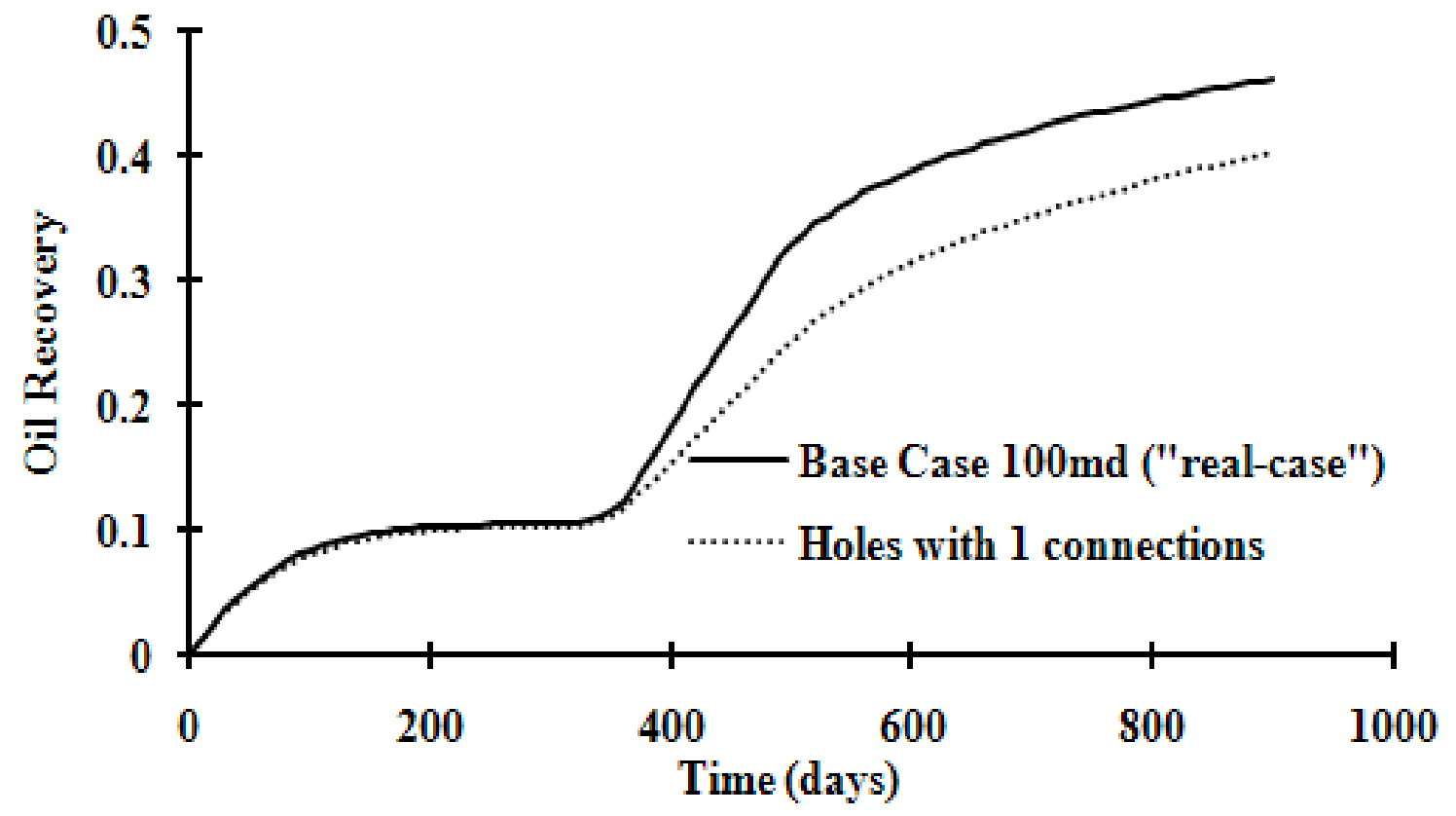

Figure 3.25 Oil recovery factor comparisons between base case and reduced connections case 
This connectivity case study simply shows the large impact of fracture network connectivity on hydrocarbon recovery, particularly for the Type I basement reservoir. When basement reservoirs are compared with other types of fractured reservoirs such as Type II or III, the matrix does not contribute any permeability to fluid flow. Therefore, in the basement type reservoirs, fluid cannot bypass discontinued fractures through the matrix. This makes it essential to study the connectivity risk model in order to understand the reservoir behavior. As addressed before, this conceptual model also shows the importance of reservoir characterization. The correct fracture network connectivity characterization will be the key to understanding the basement reservoirs.

\section{Fracture Orientation Impacts}

As classified above, for the basement type reservoir, all the reservoir fluids exist only inside of fracture/fault. Since the orientation has always been an important characteristic for the fracture existence, it is instructive to quantify the impacts on hydrocarbon productions due to the fracture/fault orientations in the basement reservoir.

Before studying fracture orientation impacts on the oil/gas recovery, it is useful to know some fracture terms. Generally, pole and dip vectors are terms used to define the orientation of a plane in space. Fractures in the DFN modeled basement reservoir are planar polygons and use poles or dips to describe their orientation. The pole is a vector normal to the fracture plane and usually points downward. The dip vector is a vector normal to the pole and lies in the plane pointing in the direction of the maximum slope gradient. The angle between the dip vector and the horizontal is called the dip angle. The direction of the horizontal trace left by a fracture/fault intersecting a horizontal plane is called its strike. The concepts of dip and strike are shown in Figure 3.26 and Figure 3.27. 


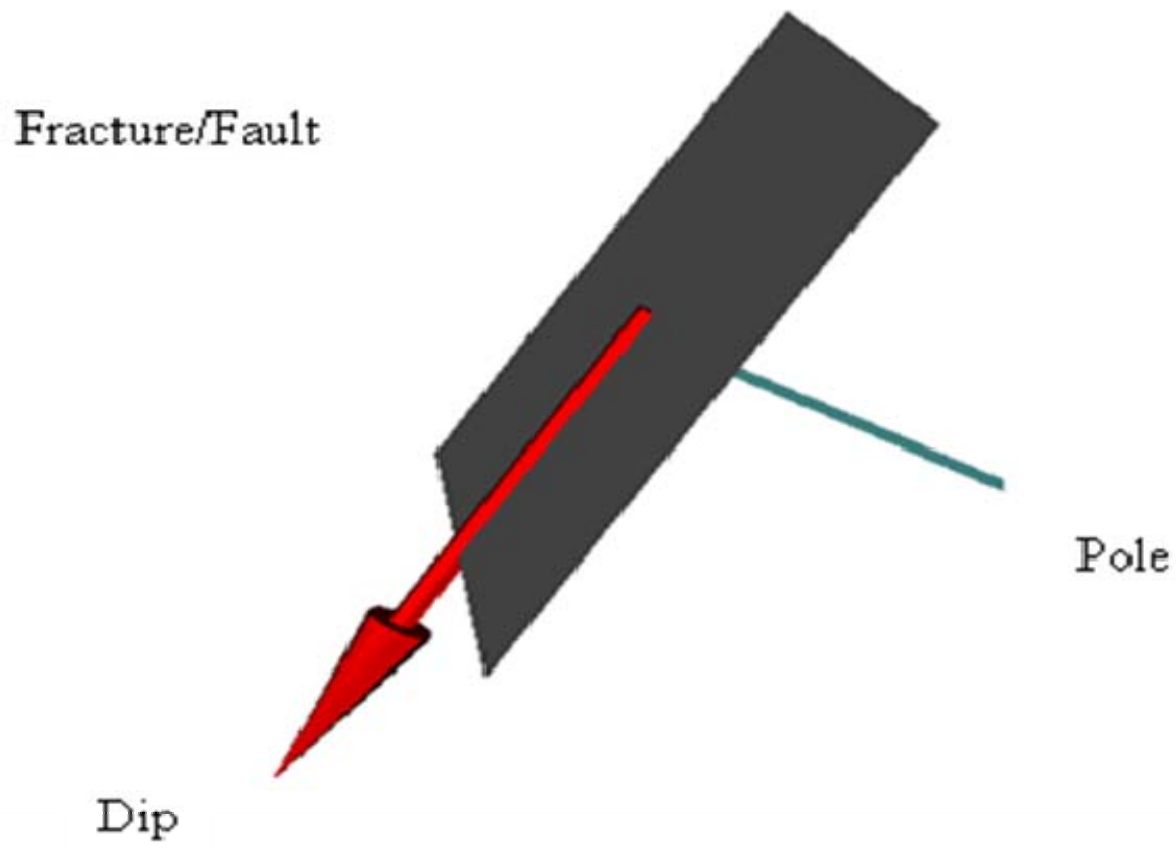

Figure 3.26 Illustration of single DFN modeled fracture orientation (pole and dip vector)

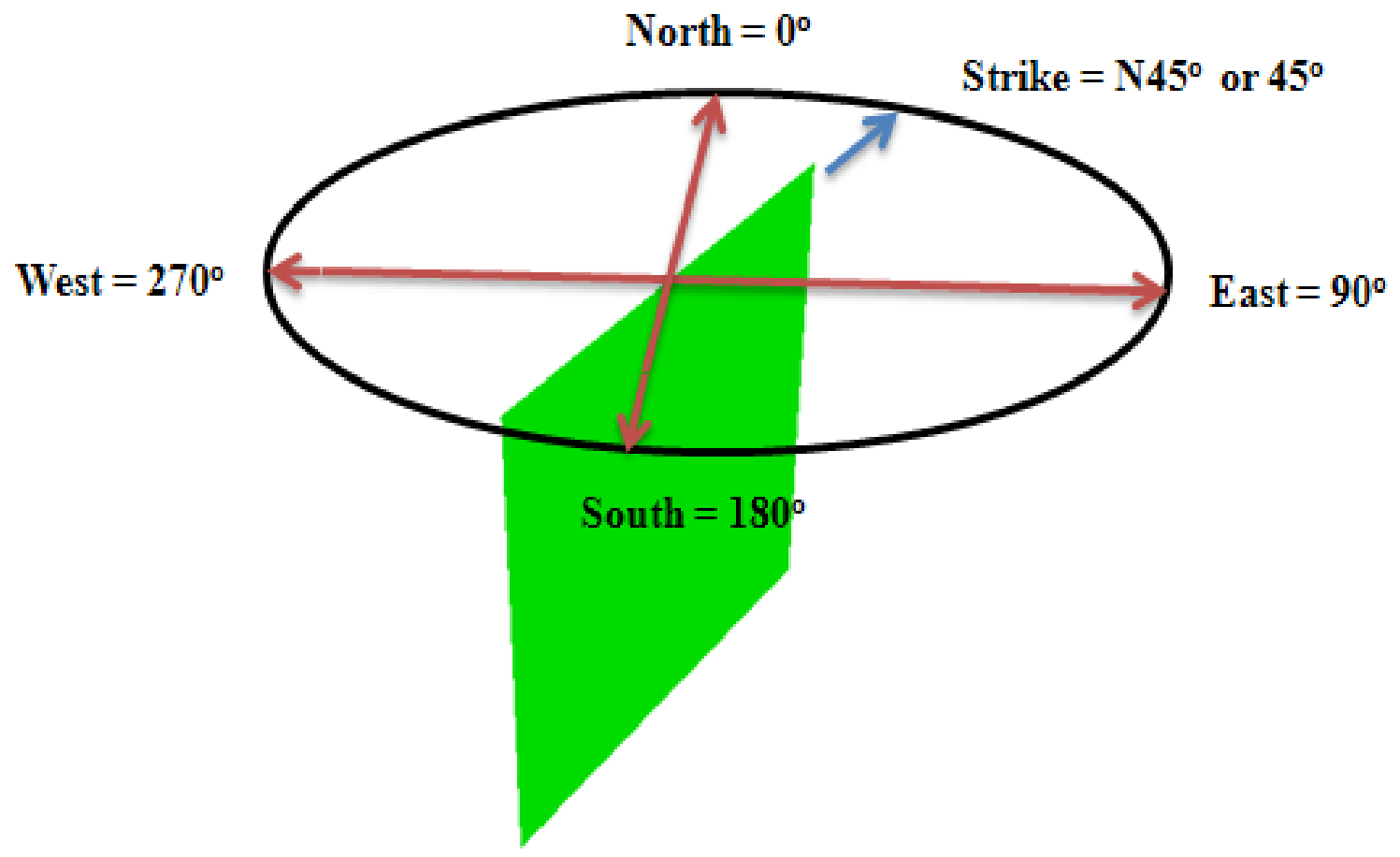

Figure 3.27 Illustration of fracture strike orientations 
The "real" basement model discussed above was characterized with the dip angle of $90^{\circ}$. In this section, the fractures/faults in the "real" basement reservoir model were identified as two sets of fractures based on their strike. The strikes from 0 to $45^{\circ}$ were identified as fracture/fault set 1 and the rest were marked as fracture/fault set 2. For fractures/faults in set 1, the dip angles were kept the same as before $\left(90^{\circ}\right)$, and the fractures/faults in set 2 were tuned into $45^{\circ}$ as their dip angles. Both the injector and the producer were located in the fracture/fault set 1 , and there was no change in their operating conditions and the fracture condition they located. This gave the smallest effects on the wells. For fractures/faults in set 2, their upper edges were exact as before but the bottom edges were in different locations based on their strike angle. The areas and shapes for all of the fractures/faults in this basement domain were kept the same which resulted in the same hydrocarbon in place as before. The hypothesis of this study was conceptually tested by the impacts of fracture orientations on the hydrocarbon recovery.

The populations of the fracture's orientation distribution could be visualized by stereo-plots. In the stereo-plots, the orientations are plotted in terms of the locations of poles. Horizontal fractures are demonstrated in the center of the stereo-plot; vertical fractures are represented on the circumference.

The stereo-plots in Figure 3.28 describe orientation differences between the base case and the tilted orientation distributions case. It clearly shows that in the tilted basement domain, there are two sets of fractures with two different pole/dip orientations (set 1 with $0^{\circ}$ of pole which is $90^{\circ}$ dip orientation and another set with $45^{\circ} \mathrm{dip} /$ pole orientation). 


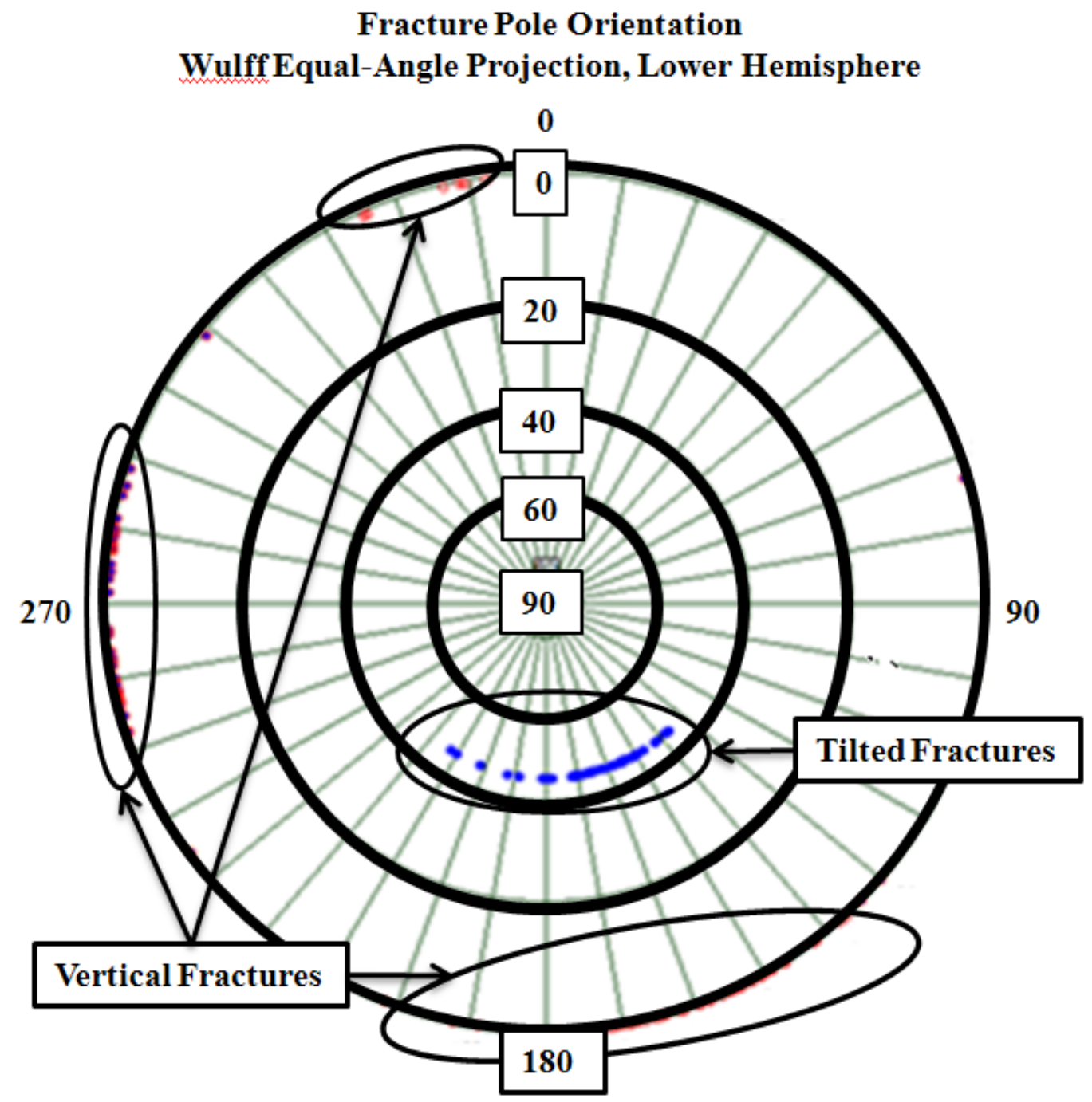

Figure 3.28 Comparisons of fracture orientation distribution between base case and titled case by stereo-plots

Figure 3.29 and Figure 3.30 show that the fracture orientation impacts the hydrocarbon production rates but not the overall recovery. The peak oil production rate in the tilted domain was about $12 \%$ higher than the base case. However, after 900 days which include primary and secondary production stages in this study, the overall oil recoveries were very close (less than $2 \%$ difference) between the all vertical fracture case and the tilted domain case. 


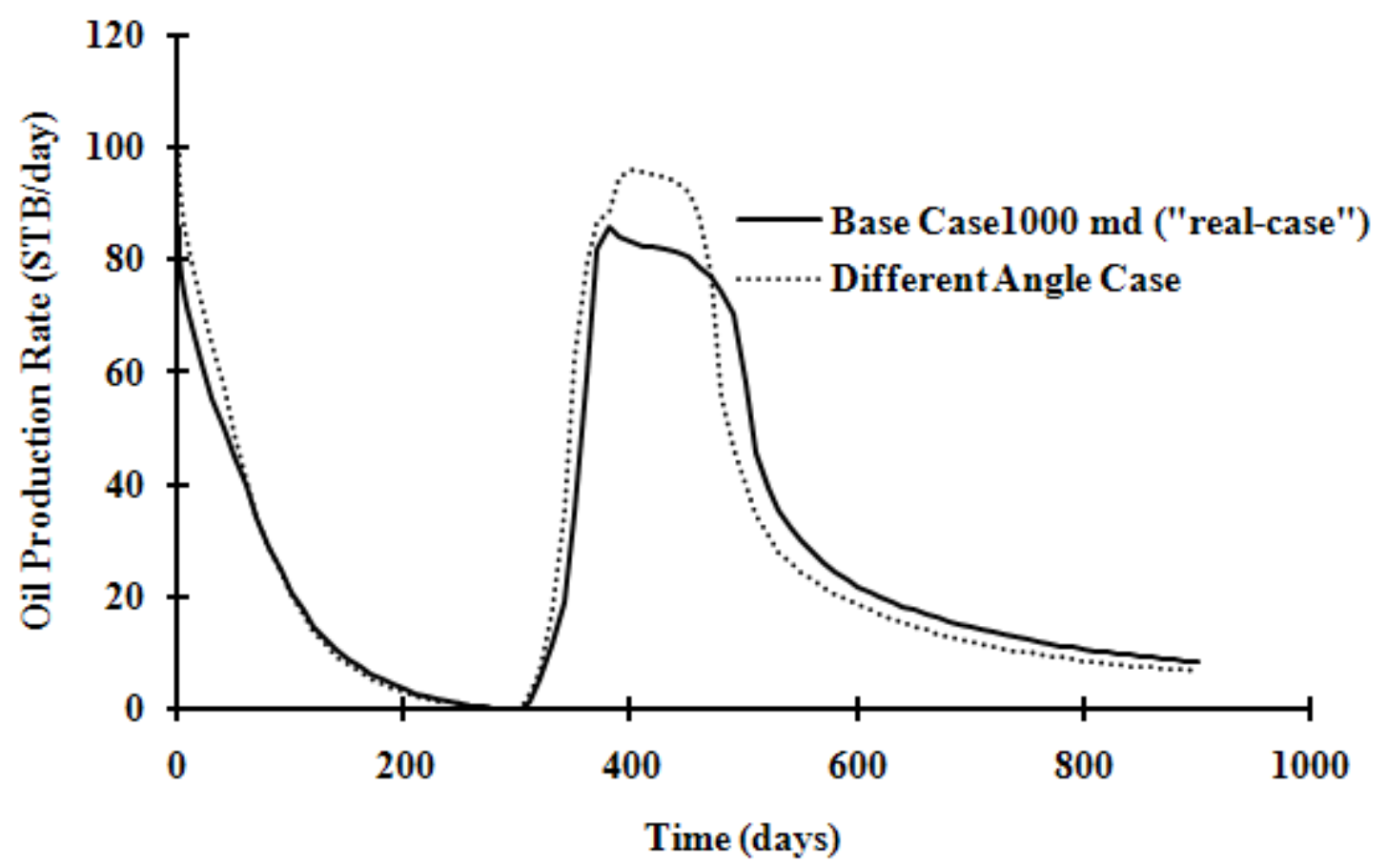

Figure 3.29 Oil production rate comparisons between the base case and the dip angle case

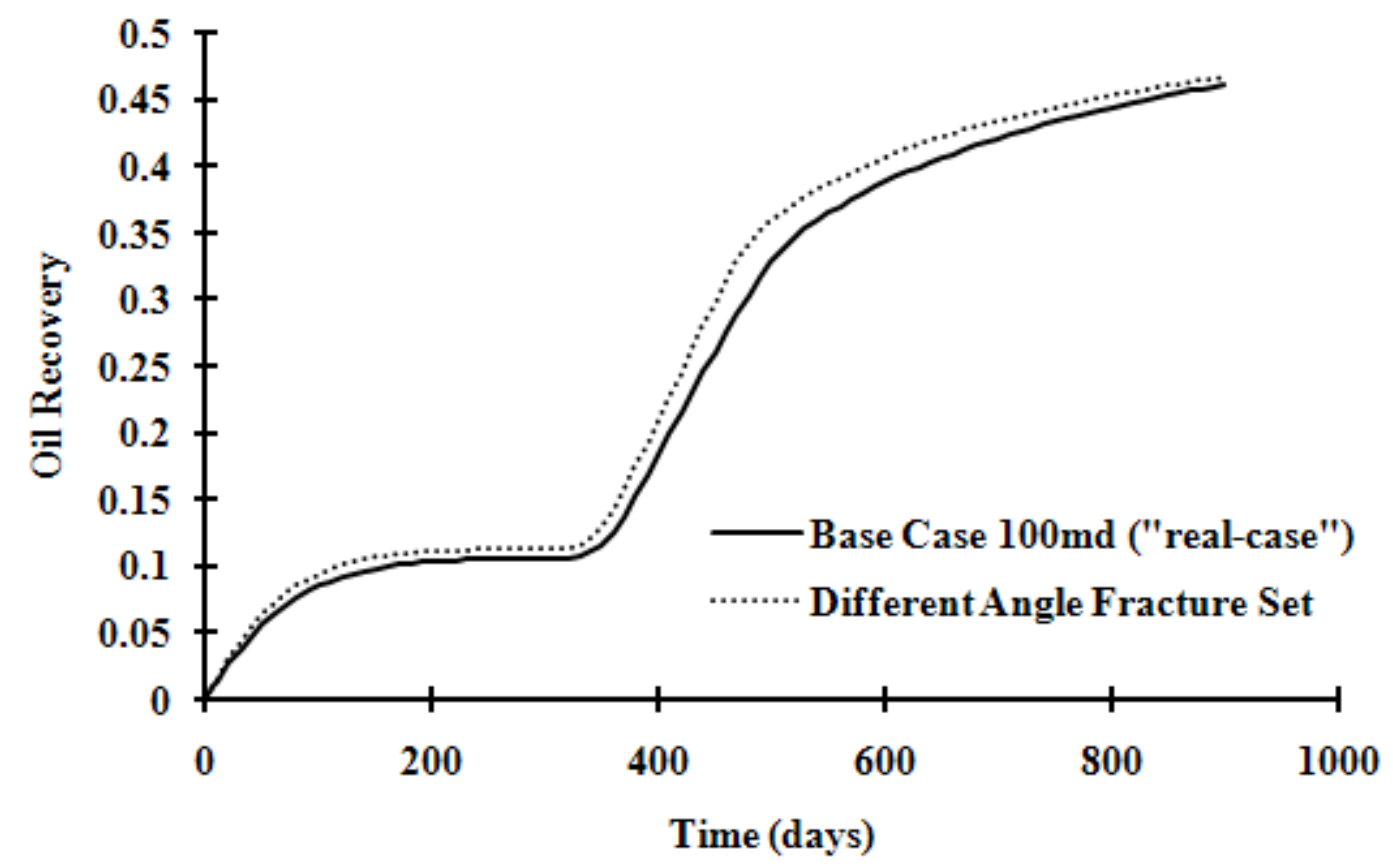

Figure 3.30 Recovery comparisons between the base case and the dip angle case 
Figure 3.31 shows that the residual oil distributions are slightly different at the end of simulation between the base case and tilted domain case study. The reason for obtaining this result might be related to tilted fracture set characterization. In this case study, fracture orientation variations result in fractures' contacts being increased slightly and making more reservoir fluid exist on slightly higher locations. This study qualitatively demonstrates that the fracture orientation can impact on oil productions.

Base Case

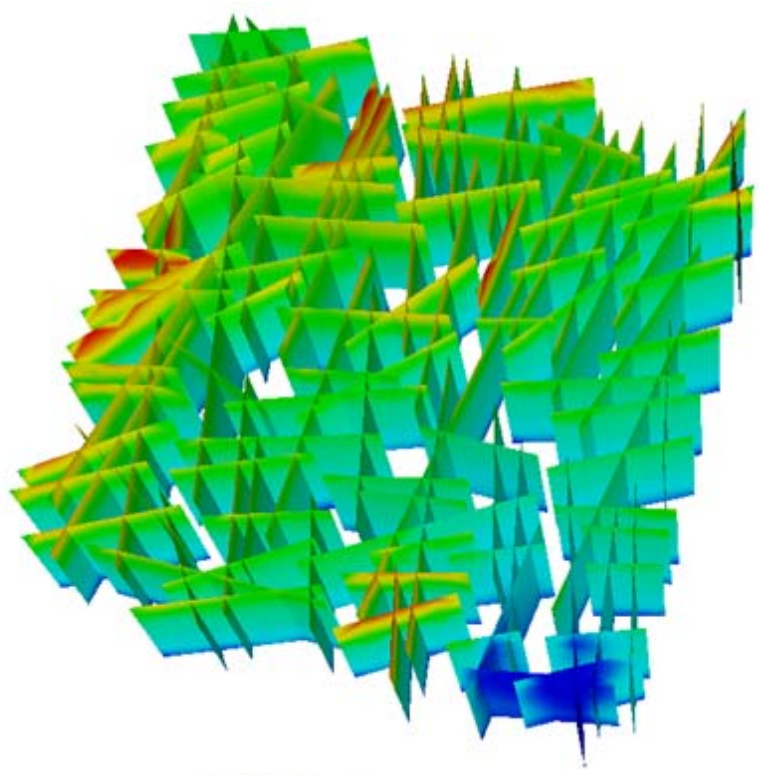

Tilted Domain

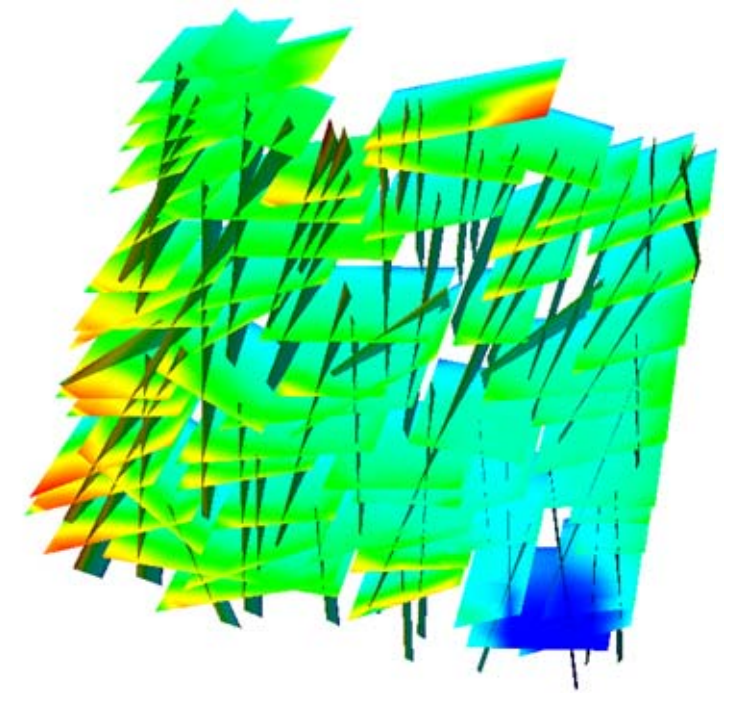

Figure 3.31 Oil concentration distribution comparisons between base case (top) and the tilted domain case (bottom) 


\subsubsection{Fracture Height Variations Studies}

Like fracture properties discussed in this chapter (connectivity, permeability), the fracture size is also an important parameter in the fracture reservoir modeling. Limited information was acquired from normal exploring results in poorly defined fracture network modeling. Thus, quantifying the uncertainty on the fracture size information becomes essential in understanding fractured reservoir behavior.

In this section, a sensitivity study of fracture length in the z-direction (vertical) was proposed on the basement reservoir. The 1000x1000x200 $\mathrm{ft}$ base case domain with $1000 \mathrm{md} k$ being studied before was still treated as the base case in this section. On the same trace map domain, two sets of fracture/fault clusters were identified by their strike angles. The strikes from 0 to $45^{\circ}$ were characterized as fracture/fault set 1 and the rest were marked as fracture set 2. For fractures/faults in set 1 , all fractures were kept the same height as originally (200 ft); the heights of fracture in set 2 were shrunk to a half of the original size $(100 \mathrm{ft})$. Two simulations were performed in this study: fracture set 2 on the top and on the bottom. As mentioned in the last application, the producer and injector were located on fractures set 1 . Therefore, nothing was changed for the fractures related to wells. To keep the same oil in place, the porosity in fracture set 2 was doubled (since the height of fracture set 2 is half as the base case). These two simulations were performed with the same reservoir fluid properties and operating conditions.

Figures 3.32 and 3.33 show that comparisons of oil production rates and oil recoveries among the studies of the base case (full fracture height), the half-top case and the half-bottom case. The comparison results are interesting: during the stage of primary production, the half-bottom case shows significantly higher rates than the other two; after the water injection since day 300 , the best oil production rate came from the base case, then second-best came from the half-top case, and the lowest rate came from the half-bottom case. Figure 3.33 shows similar trends as shown 
in the rate figure. However, at the end of simulation (day 900), both "half-" case studies almost reached the same hydrocarbon total production $(1.40 \%$ difference). The base case with full fracture/fault heights offered $30.66 \%$ higher oil production than the half-bottom case.

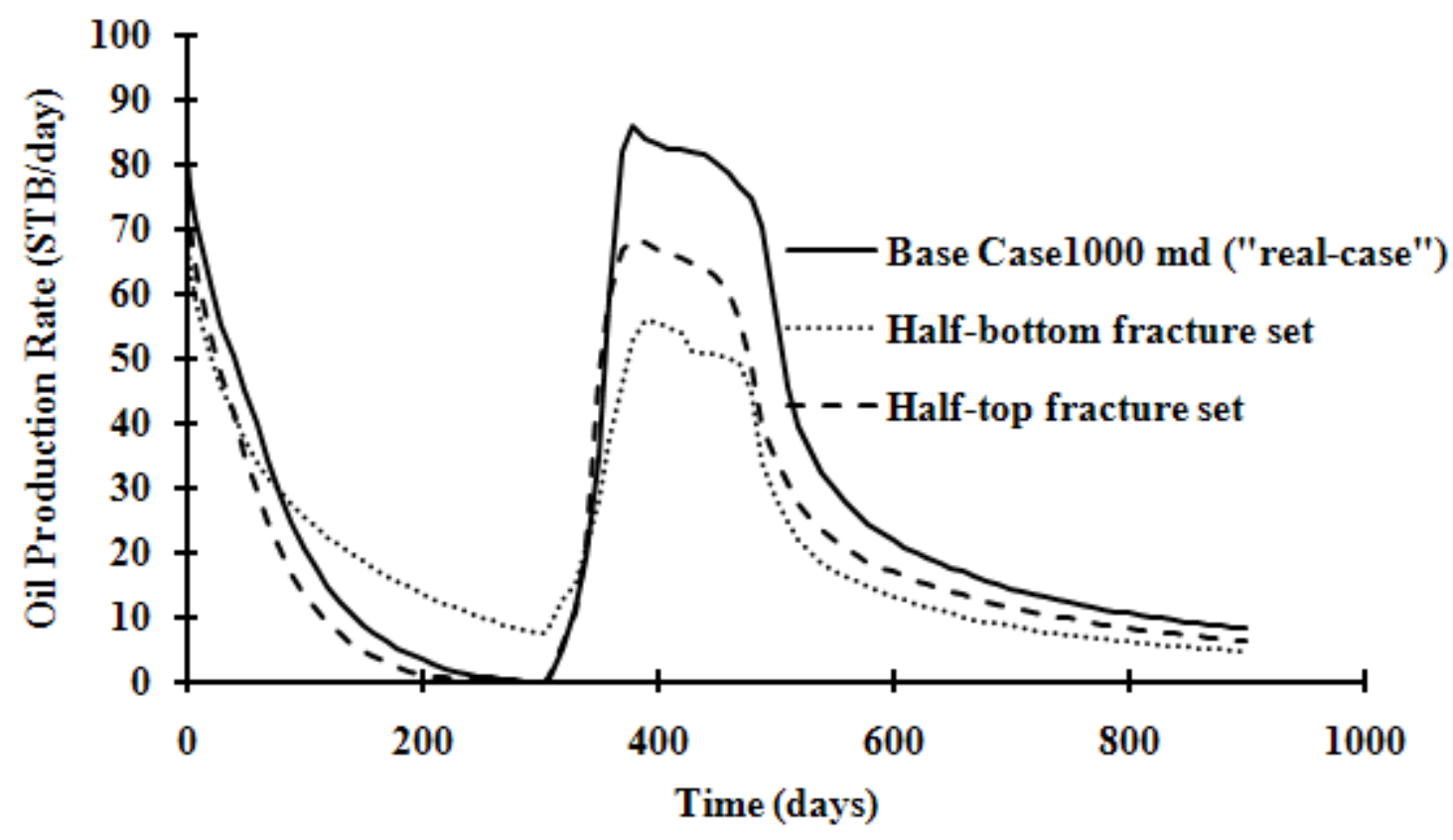

Figure 3.32 Oil production rate comparisons among base case (full fracture height), half-top case and half-bottom case with same oil in place and well properties 


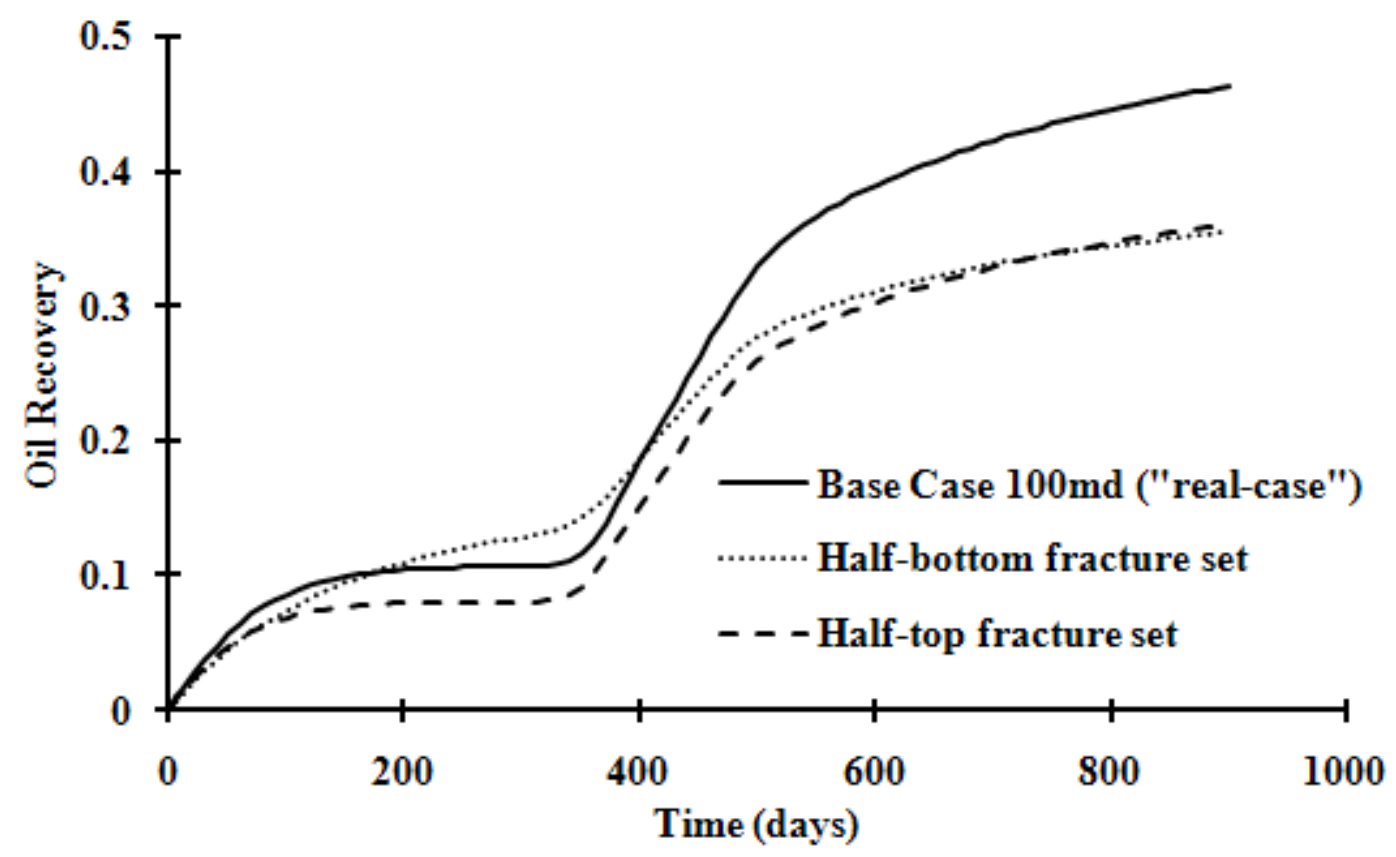

Figure 3.33 Oil recovery comparisons among base case (full fracture height), half-top case and half-bottom case with same oil in place and well properties

Figures 3.34 and 3.35 demonstrate the reason for higher oil production rate from the half-bottom case: during the primary oil production stage, reservoir energy (pressure) is the only driving force producing hydrocarbons. For this three-phase black oil model, the production well was located on the corner of the upper-left fractures and operated with bottom-hole pressure (BHP) control, which was higher than the bubble point pressure. The reservoir pressure decreased as oil was produced. The gas-phase was gathered on the top of the reservoir. At the end of primary production, the oil saturation from the base case and the half-top case showed close, even distributions. However, only half of the fractures in the half-bottom case were characterized on the top of the reservoir; the gas-phase moves to the top through fractures with full-height fracture set (set 1) in this case. Since the original oil in place was the same for all three cases, the fracture vertical size difference resulted in higher concentration with lower fracture numbers on the top of the reservoirs. Figure 3.35 shows at the day 300 (end of primary production), no oil is produced 
from the base case and the half-top case. The half-bottom case still has enough reservoir energy to produce at that time. This explains the significantly higher rates from the half-bottom case study at the end of primary production. The rate difference between the other two cases (the halftop and the base cases) during the primary production could be explained as the result of more fracture connection areas in the base case: when pressure decreases, gas-phase in the oil phase goes to the reservoir top, and full height fractures offer more connection areas and thus more potential to make fluid flow.

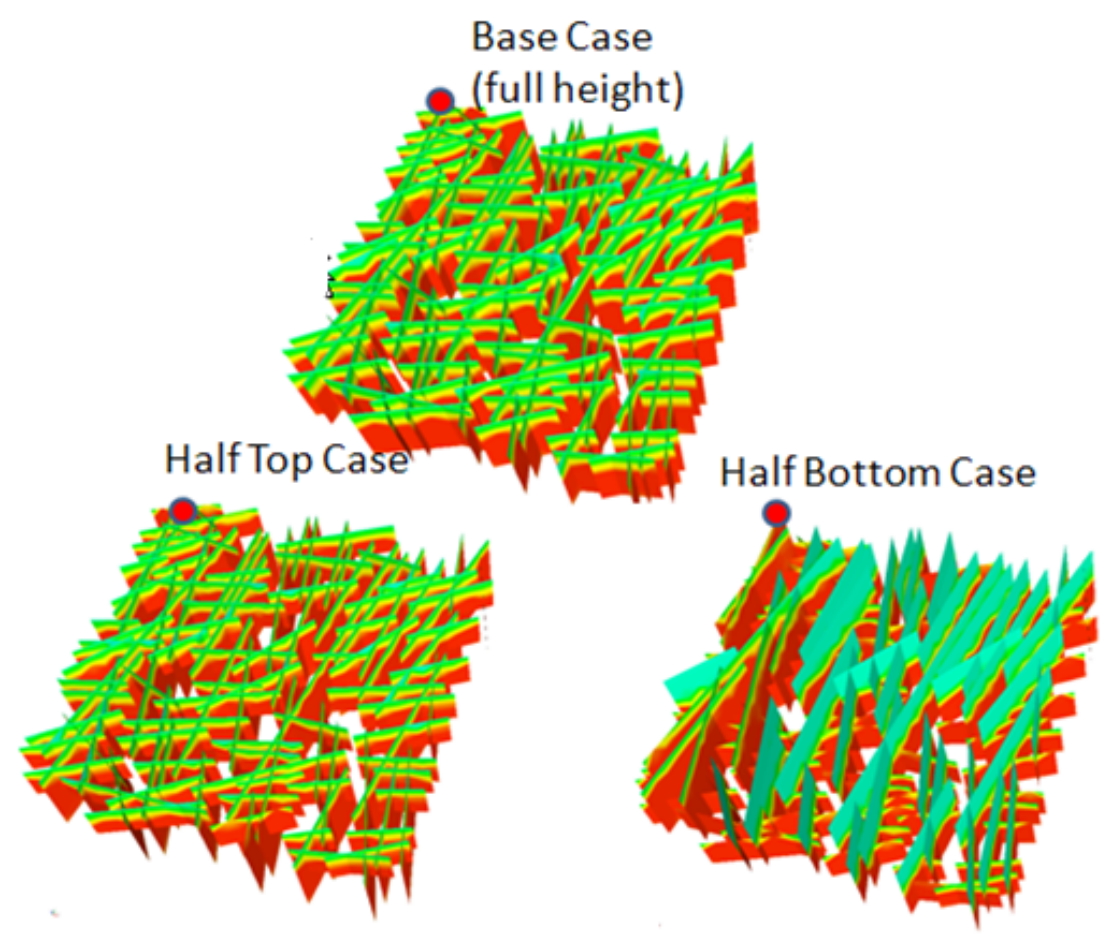

Figure 3.34 Oil saturation distribution comparisons at the end of the primary production (day 300) 


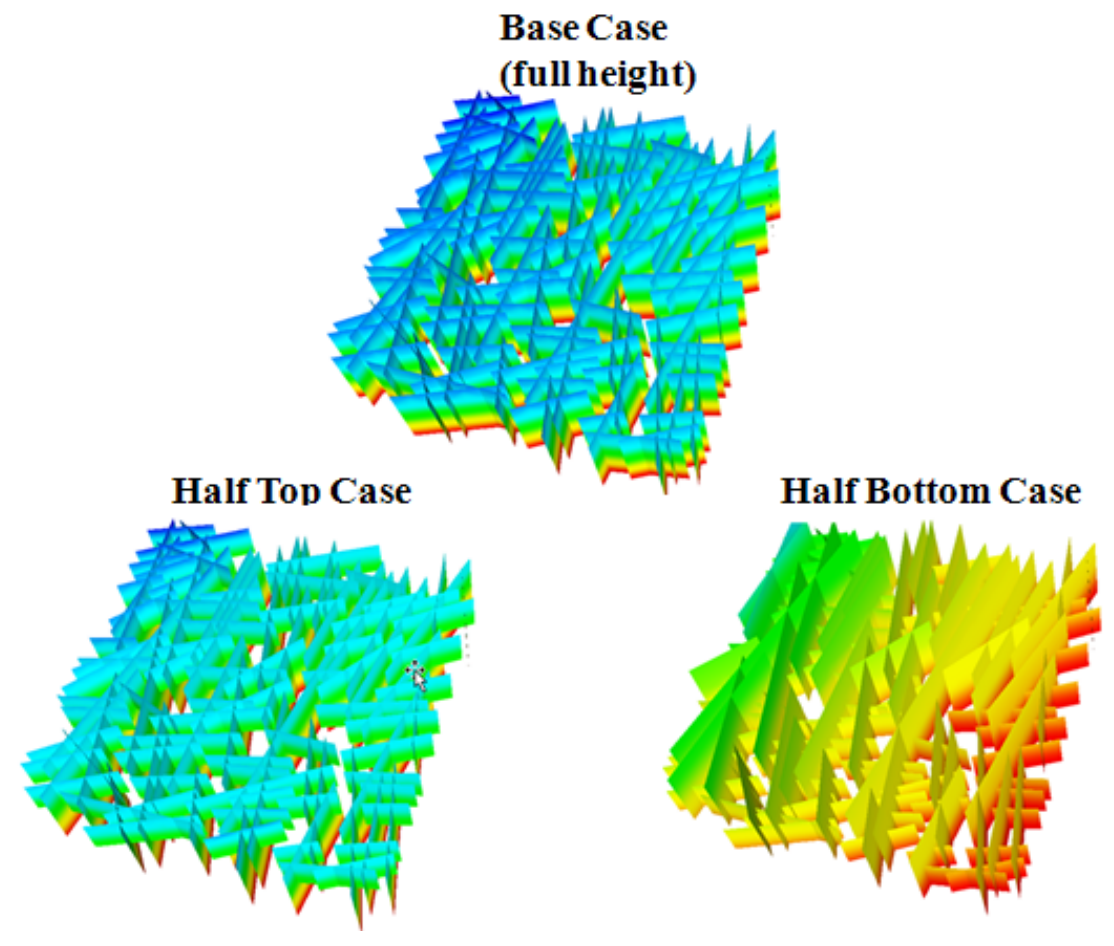

Figure 3.35 Pressure distribution comparisons at the end of the primary production (day 300)

The secondary productions were started from day 301 and lasted until the end of simulation (day 900). The water cut comparisons are shown in Figure 3.36. 


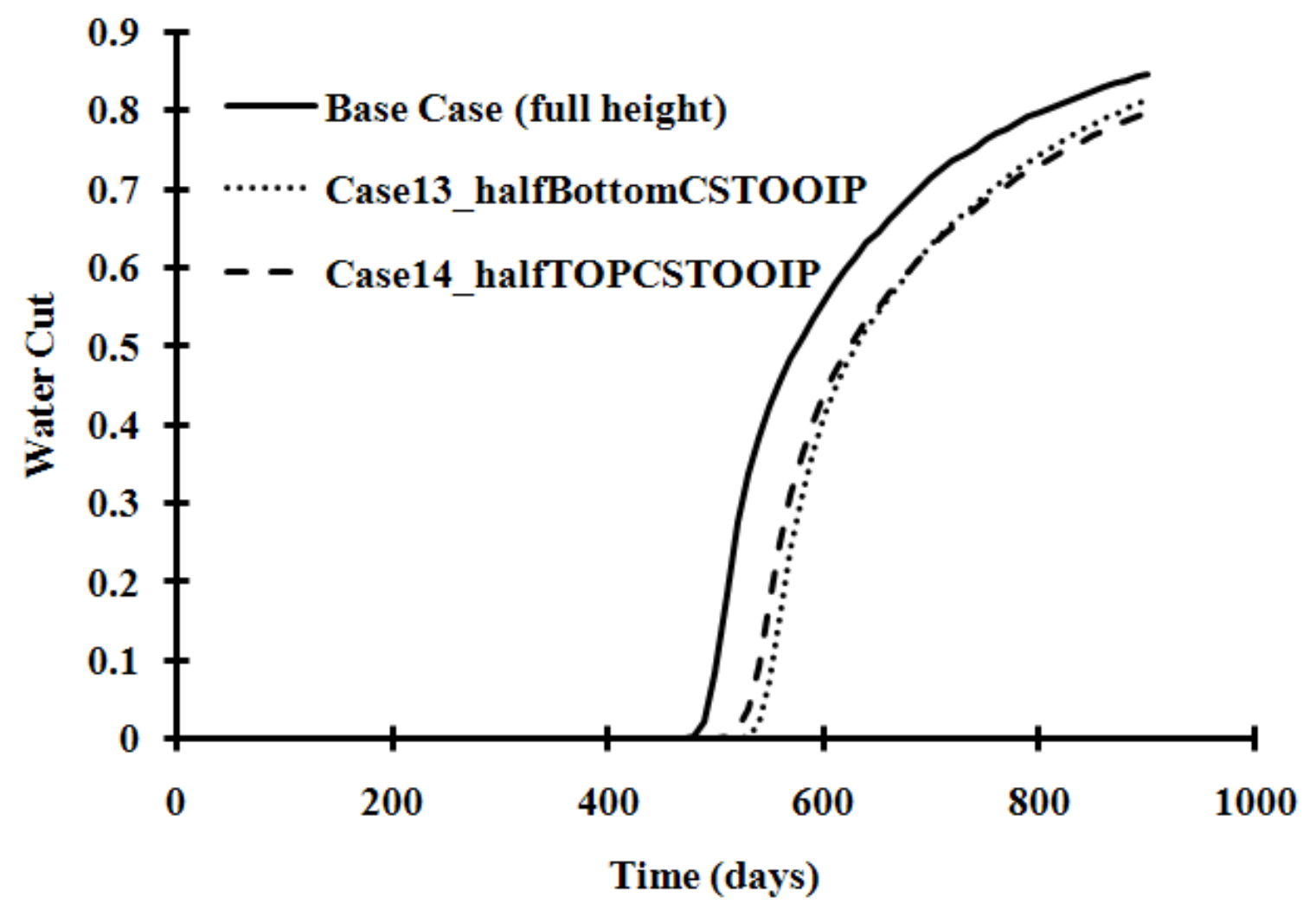

Figure 3.36 Water cut comparisons among base case (full fracture height), half-top case and halfbottom case with same oil in place and well properties

Figure 3.36 shows that the base case had the earliest breakthrough, and the other two cases had the similar breakthrough. At the end of simulation (day 900), the water cut values in three simulations were: base case (0.85); half-top case (0.81) and half-bottom case $(0.80)$.

Figure 3.37 show that the injected water sweeps the fracture network from the bottom first due to gravity effects. The residual oil (red) remained in the fracture set 2 (with strike larger than $45^{\circ}$ ) on the west side of the fracture networks. The base case has moderate residual concentrations, the half-top case has the strongest residuals, and the half-bottom case almost gets those residuals swept out. This could be explained as the better connectivity (more connection areas) for the base case with full fracture heights. 


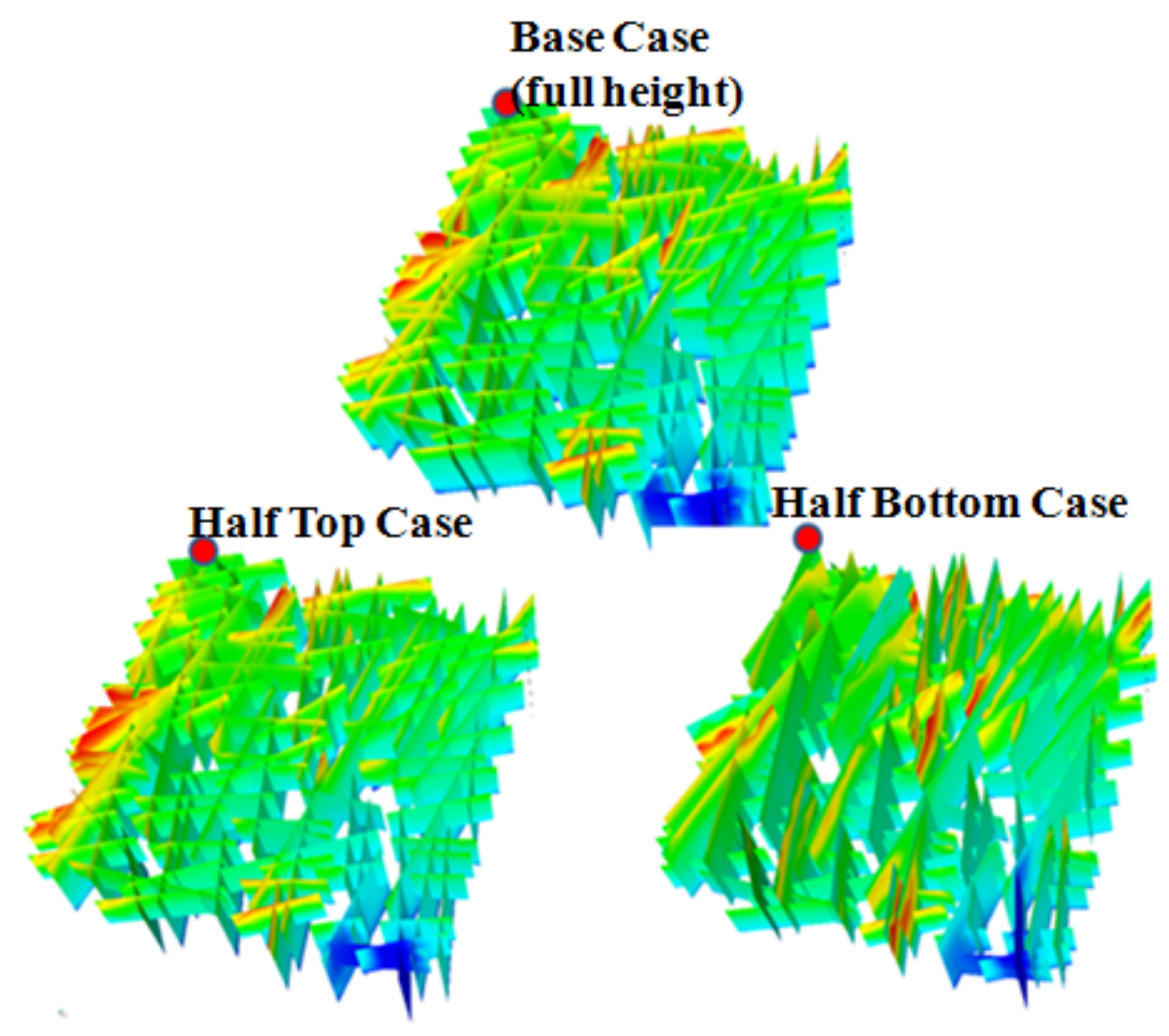

Figure 3.37 Oil saturation distributions at the end of simulation (day 900)

Some conclusions can be drawn through the studies in this section:

1) Fracture size does play an important role in the hydrocarbon recovery process, at least in the size variations in vertical fractures/faults. It impacts the water flooding patterns and residual hydrocarbon distributions.

2) Lower vertical fracture height resulted in higher hydrocarbon production rate during the primary production and lower hydrocarbon production rates (if producers are located on the top of reservoir fractures) during secondary production.

3) Connectivity is still a big issue for fractured reservoirs. In the studies in this section, the fracture connection area shows clear differences during the hydrocarbon recovery. 


\section{Preferred Pathway Studies}

It is known that a typical fracture network is highly heterogeneous. If the production and injection wells happen to be set up with the connection of short-circuit fracture networks (with much higher permeability than the rest of the fractures in the basement case), unexpected early breakthroughs will be revealed. This could make the most of the hydrocarbon residence inside of the reservoir and cannot be swept out or it takes a much longer time and more water to produce the hydrocarbon. This phenomenon can also be called the "preferred pathway" in the process of oil production. The preferred pathway can lower oil recovery efficiency dramatically. From the point of view of reservoir management, this is not preferable in any sense. Generally, if this preferred pathway existed, some techniques need to be taken to improve the oil recoveries. One typical solution is to use gel treatments to shut off the high permeability features.

In this section, to understand the impact of the preferred high permeability pathways phenomenon and the impact of shutting it off, three new simulations are characterized below:

1) Base case study: all permeability equals $100 \mathrm{md}$

2) Preferred pathway study (as shown in Figure 3.38):

a. Permeability on preferred pathway: $100,000 \mathrm{md}$

b. Permeability on the rest of the reservoir: $100 \mathrm{md}$

3) Preferred pathway with cut-off zones:

a. Permeability on preferred pathway: $100,000 \mathrm{md}$

b. Permeability on preferred pathway cut-off zones: $0.1 \mathrm{md}$

c. Permeability on the rest of the reservoir: $100 \mathrm{md}$ 


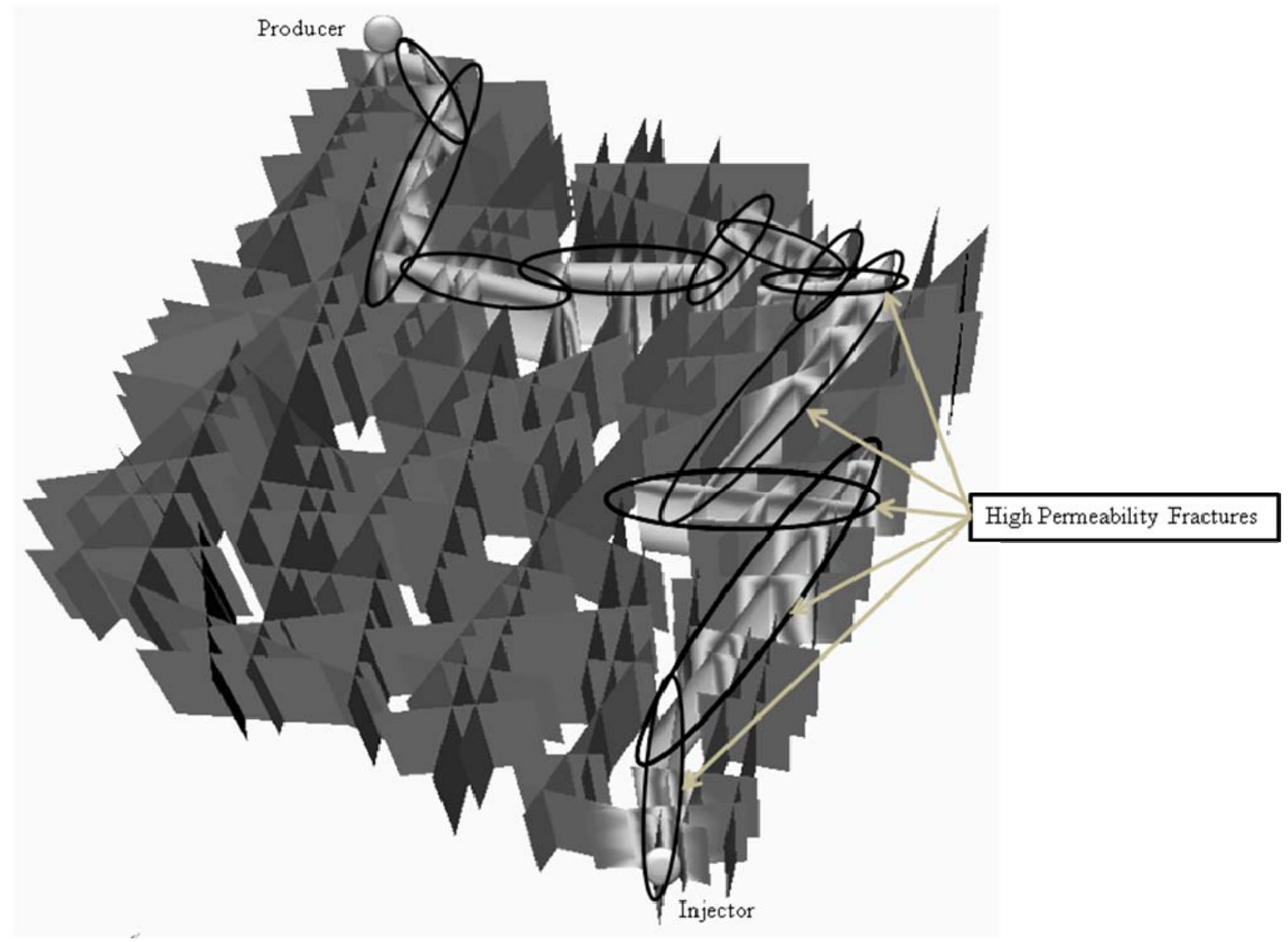

Figure 3.38 Illustration of preferred pathway characterization (other than circled area) and preferred pathway with cut-off zone (circled).

4) Other reservoir information:

a. Pore volume: $67287 \mathrm{STB}$

b. OOIP: $52830 \mathrm{STB}$

c. Phase: two phases (oil and water)

In this two-phase preferred pathway study, injection was started from day 1 . The base case fracture permeability was characterized as $100 \mathrm{md}$. Both the injector and the producer were controlled by bottom-hole pressure control. Other than the permeability variations, all conditions in three models were the same. The results of oil saturation distribution on different times (day 10, 100, 1000 and 8000) are shown in Figure 3.39. 
Figure 3.39 shows totally different water flooding patterns from three models. As shown in the figure, the results from different simulations vary by columns and the output times vary by rows. The first row demonstrates the oil saturation distributions at the $10^{\text {th }}$ day. At that time, the preferred pathway simulation in the middle of the row shows a clear water breakthrough path between the producer and the injector. The base case has only a small area affected near the injector; the cut-off zone case shows stronger water effect than the base case but not as much as the preferred pathway case. This is from a couple of short, high permeability features which still exist near the injector. At day 100, Figure 3.39 shows a much clearer effect among different cases: the water flooding path is equally distributed on the base case, but there is a high water cut on the preferred pathway case. The cut-off zone study still shows the greatest water-affected areas, but the feature with gel treatment shows a stronger barrier effect. On day 1000, the oil in this basement reservoir model had been recovered as base case $(0.45)$; the preferred pathway case (0.27), which is $40 \%$ lower than base case; and preferred pathway with cut-off zone (0.51), which is $13 \%$ higher than base case. At the end of the simulation runs (day 8,000 ), results of oil recovery rates are base case $(0.63)$; preferred pathway case $(0.58)$, which is $8 \%$ lower than base case; and cut-off zone case (0.70), which is $11 \%$ higher than base case. The residual oil saturation distribution at the end of the simulations has totally different patterns. Base case has the most even water flooding between injector and producer, preferred pathway simulations gives large amounts of higher concentration residual oils on the southwest part of the reservoir; and cut-off zone simulation offers the smallest amount of residual oil left in the reservoir with the highest residual oil remaining in the gel-treated cut-off features. Comparisons of oil recoveries for preferred pathway study are shown in Figure 3.40. 

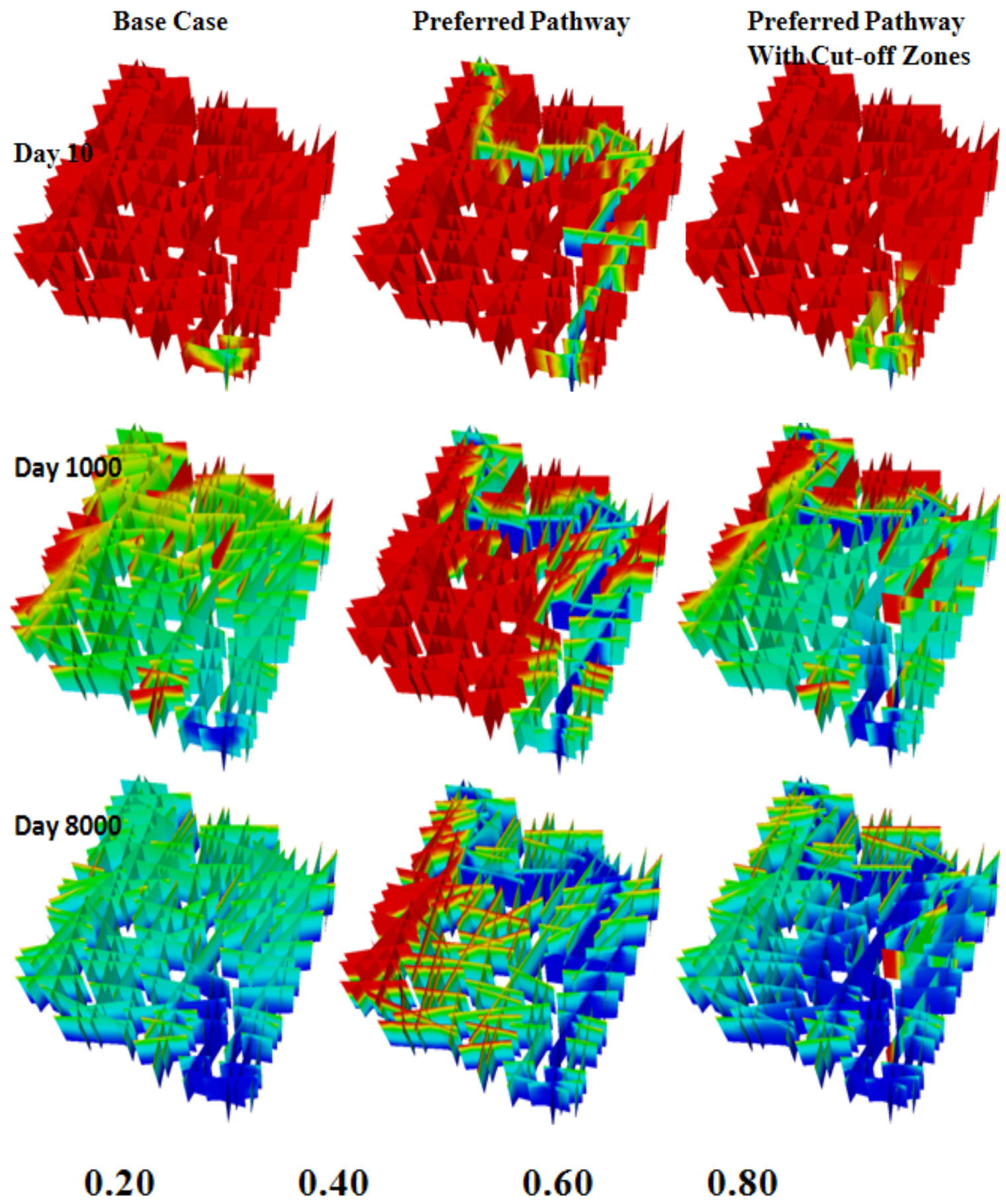

0.40

0.60

0.80

So

Figure 3.39 (continued) Oil saturation distribution comparisons among different fracture permeabilities (base case, preferred pathway, and preferred pathway with "cut-off zone") 


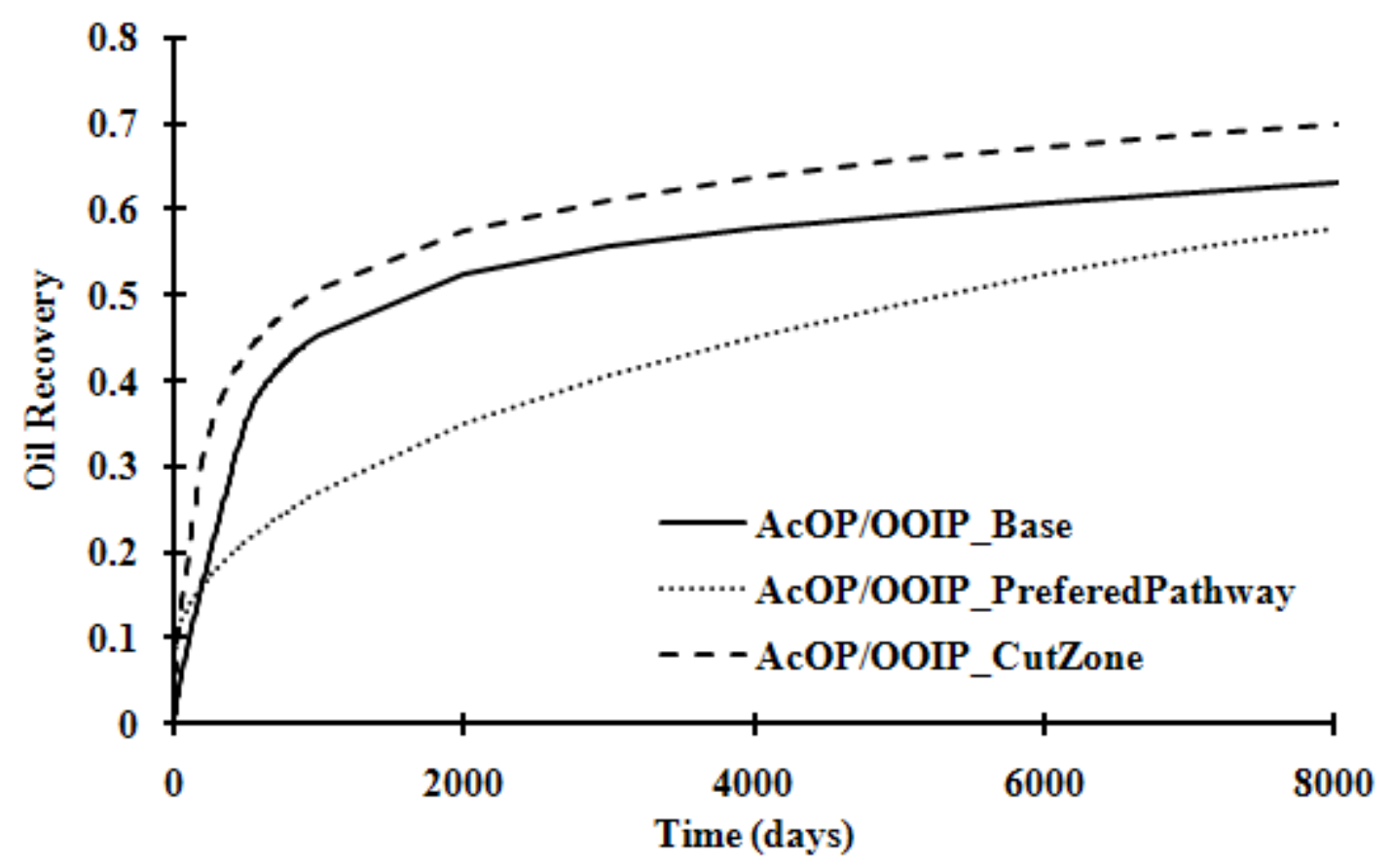

Figure 3.40 Comparisons of oil recoveries for preferred pathway study

Figure 3.40 shows that at the beginning of the simulations, for a short period, both the preferred pathway and the cut-off zone cases have identical oil recovery rates, which are higher than base case. This is due to the existence of higher permeability fractures near the injector. Then the trend of oil recoveries in Figure 3.40 displays the recovery with the order from high to low as: cut-off zone case, base case and preferred pathway case. This result is encouraging for the idea of treating higher permeability fractures with special techniques such as gels in order to lower or block the water breakthrough. The treatments can dramatically increase the oil recovery. For example, to reach $40 \%$ oil recovery, the homogeneous permeability base case required 660 days, the preferred pathway case required about 2900 days, and cut-off zone case required only 382 days. There is nearly one magnitude difference before and after the treatment.

To analyze the relationship between recovered oil and injected/produced water, some dimensionless analysis is demonstrated in Figure 3.41. 


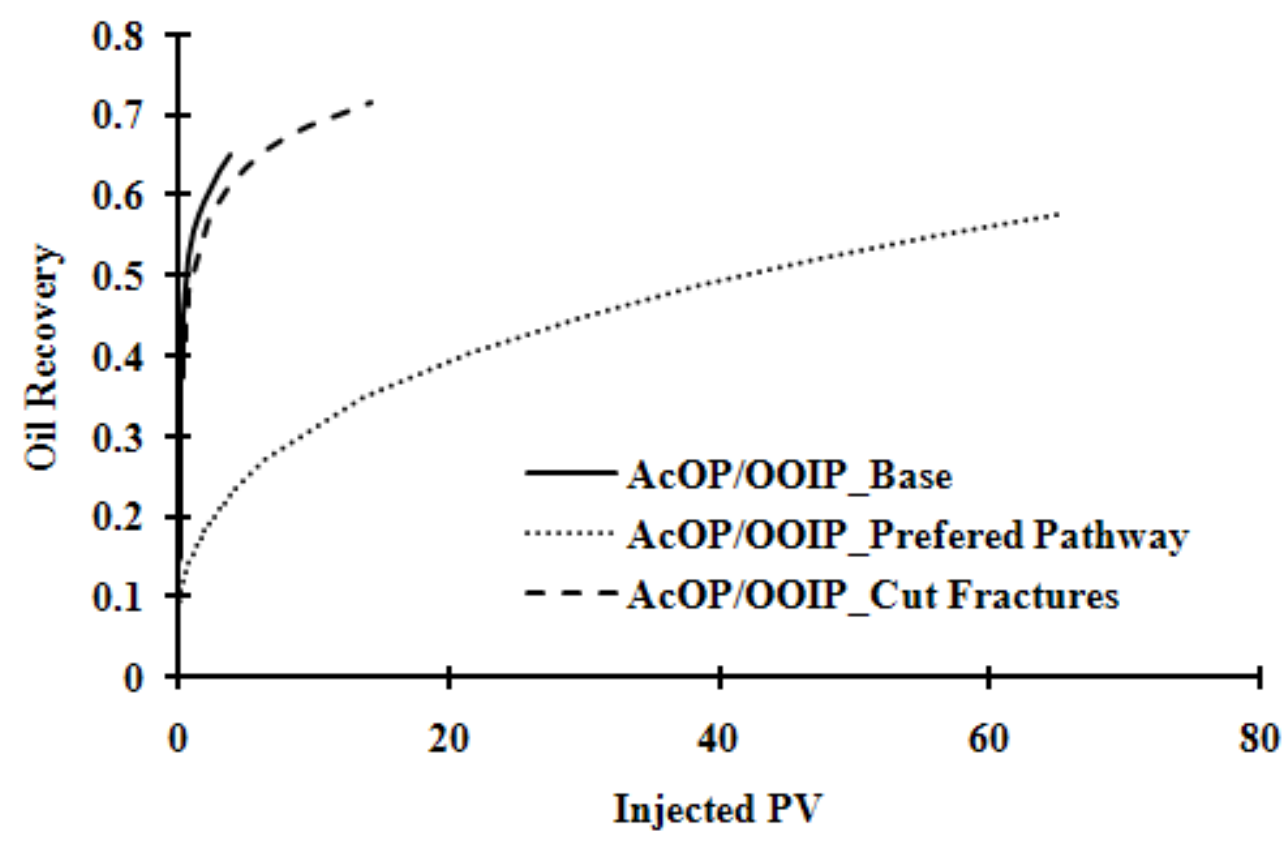

Figure 3.41 Dimensionless analysis of oil recovery vs. injected pore volume

The correlations between recovered oil and injected/produced water in Figure 3.41 is interesting: to reach $40 \%$ oil recovery, the base case required 0.32 volume of PV (reservoir pore volume) water injected, the preferred pathway case needed $21.68 \mathrm{PV}$ water; and the cut-off zone case needed 0.40 PV water. The difference between injection water required for the preferred pathway and the cut-off zone (for $40 \%$ recovery) is more than 54 times of PV. From another point of view, with equal PV water being injected, the base case gave $54 \%$ recovery, the preferred pathway case offered $15 \%$ oil recovery; and the cut-off zone case resulted in $50 \%$ oil recovery. The recovery difference between the preferred pathway and the cut-off zone models is $35 \%$ of OOIP.

The recovery-produced water correlations are shown in Figure 3.42. For $40 \%$ target oil recovery, the base case produced $0.017 \mathrm{PV}$ water, the preferred pathway case produced $26.3 \mathrm{PV}$ water, and the cut-off zone model produced $0.146 \mathrm{PV}$ water. The produced water volume between the 
preferred pathway model and the cut-off zone model gave more than 26 PV difference in this scenario. The preferred pathway model produced more than 180 times more water than the cutoff zone model. If the reservoir produced one time of PV water cumulatively, then the base case had $57 \%$ oil flooded, the preferred pathway model had $15 \%$ oil recovered, and the cut-off zone model had a $51 \%$ oil recovery rate. Therefore, one time of PV cumulative water production between the preferred pathway model and the cut-off zone model results in a $36 \%$ difference in original oil recovery.

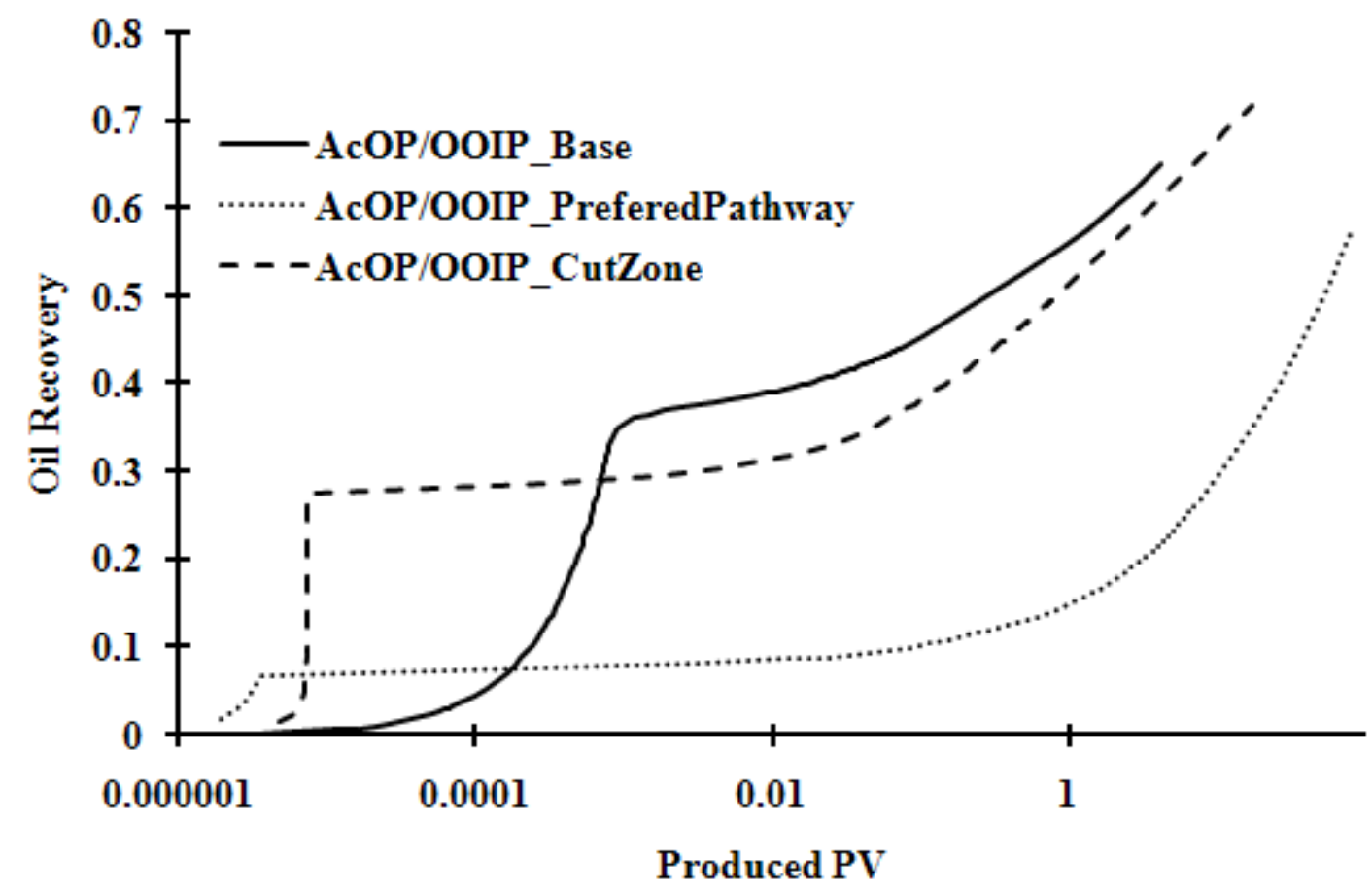

Figure 3.42 Dimensionless analysis of oil recovery vs. produced water

The following points from this section could be highlighted:

1) If injectors and producers are linked by ultra high permeability fracture networks, limited water flooding efficiency can be expected. 
2) The method of using gel or blocking the main preferred pathway fracture/faults can dramatically eliminate the negative effects caused by the ultra high preferred pathway. The advantages include higher recovery rate, lower injection-water demand, lower produced water-treatment cost (can reach two magnitude difference or more), etc.

\section{Chapter Summary}

At the beginning of the chapter, a regularized fracture network was simulated with ECLIPSE (a well-established commercial reservoir simulator) and CVFE (an in-house DFN simulator developed by the University of Utah). The results of the two simulations were compared, and they showed that both simulators were in close agreement, even under the three-phase conditions.

Sensitivity case studies in this chapter demonstrated the importance of fractured reservoir characterization work. A precisely characterized fracture network could reduce the reservoir production uncertainty risk significantly. Quantitatively, this chapter discussed the effect of different fracture properties on multiphase flow performance in a "real" basement fracture model. This "real" fracture network was difficult to simulate by conventional finite-difference simulators such as ECLIPSE. The following conclusions can be drawn from this study:

1) When a random permeability distribution was characterized for the network, the flow performance was dominated by the presence of the low permeability features. The permeability distribution also impacts oil recovery and water cut.

2) The DFN approach allows us to cut off main connections in the flood pathway (between injector and producer) and evaluate those cut-off impacts. It was shown that primary production was not impacted significantly by the disturbance in connectivity, but water flood performance was. 
3) Changing the fracture's dip orientations resulted in production rate variations but did not impact the overall recovery significantly.

4) When the fracture height was reduced in one of the two intersecting networks, the oil recovery was significantly lowered.

5) The DFN approach also allowed us to evaluate "what-if" scenarios. If one of the main pathways between the injector and the producer has inherently higher permeability (or an induced high permeability), then water channels through this pathway lower recovery by as much as $10-15 \%$. By blocking the preferred pathway, water can be diverted into other parts of the reservoir, increasing oil recovery.

\section{Chapter Nomenclature}

\begin{tabular}{|c|c|c|}
\hline$B$ & $=$ & formation volume factor \\
\hline$e$ & $=$ & fracture/fault storage aperture, $\mathrm{ft}$ \\
\hline$g$ & $=$ & gas phase \\
\hline$i$ & $=$ & $i^{\text {th }}$ discretized fracture elements \\
\hline$k$ & $=$ & permeability tensor, md \\
\hline$k_{r}$ & $=$ & relative permeability \\
\hline$n$ & $=$ & number of discretized fracture elements \\
\hline$o$ & $=$ & oil phase \\
\hline$p$ & $=$ & fluid pressure, psi \\
\hline$q$ & $=$ & flux vector \\
\hline$R_{S}$ & $=$ & gas-oil ratio, MSCF/STB \\
\hline$S$ & $=$ & phase saturation \\
\hline$u$ & $=$ & velocity, ft/day \\
\hline
\end{tabular}




$\begin{array}{lll}w & = & \text { water phase } \\ \Phi & = & \text { porosity } \\ \rho & = & \text { density, } \mathrm{lbm} / \mathrm{ft}^{3} \\ \mu & = & \text { viscosity, } \mathrm{cp}\end{array}$

\section{Chapter Bibliography}

Fu, Yao, "Multiphase Control Volume Finite Element Simulations of Fractured Reservoirs," Ph.D Dissertation, University of Utah, Salt Lake City (2007)

Fu, Y., Yang, Y-K., and Deo, M., "Three-Dimensional, Three-Phase Discrete-Fracture Reservoir Simulator Based on Control Volume Finite Element (CVFE) Formulation”, paper SPE 93292, (2005)

Yang, Yi-kun, Finite-element Multiphase Flow Simulation, Ph.D Dissertation, University of Utah, Salt Lake City, UT (2003) 


\section{Chapter 4. Comparison of Discrete Fracture Network Models with the Homogenized Equivalents}

\section{Table of Contents}

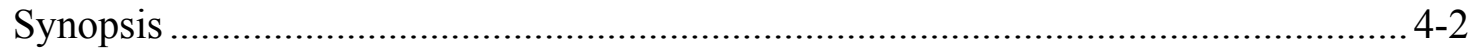

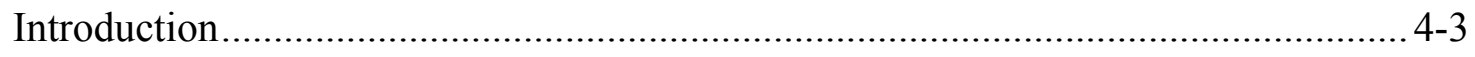

Simulating Fractured Reservoirs..................................................................... 4

Proposed Domain Characteristics ...................................................................... 4-5

Study of Hypothetical "Real" Basement Reservoir Model.......................................... 4-7

Effect of Fracture/Fault Orientation and Connectivity ............................................. 4-8

Representation of DFN Modeled Basement Fracture Network Using a Finite Difference Based

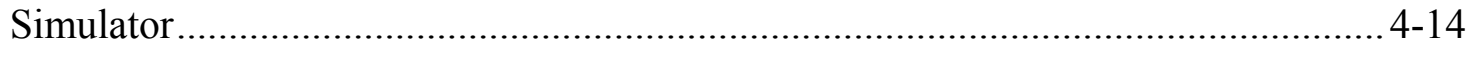

Grid Block Porosity …………………....................................................... 4-15

Grid Block Permeability ............................................................................... 4-16

Case Studies on Simulating a "Homogenized DFN Basement"................................. 4-20

Continuum Single Porosity Model by Conventional ............................................... 4-20

Finite-Difference Simulator ECLIPSE .............................................................. 4-20

Grid Block Sensitivity Studies with Porosity and Permeability Homogenization 4-20

Fine Grid for Conventional Finite-Difference Simulator of ECLIPSE .................. 4-25

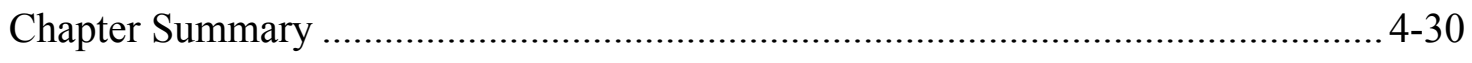

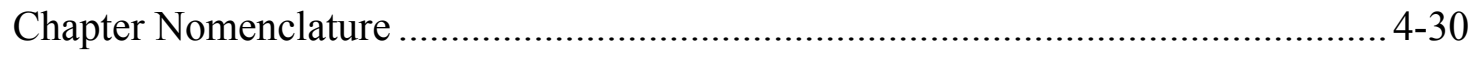

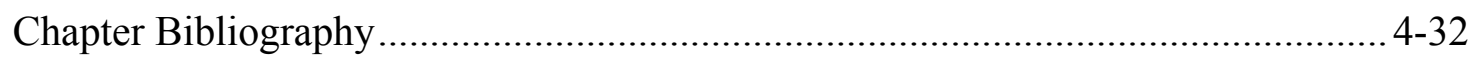




\section{Synopsis}

Naturally fractured reservoirs are an important, but difficult-to-manage, worldwide reservoir type. Complex and difficult to define, fracture networks yield reservoir systems that can be important assets for oil companies, yet the ability to improve production in these reservoir systems is hampered by their complexity. Despite the key role of fracture networks in production performance, reservoir simulations typically use equivalent porous medium properties to represent the aggregate impact of fracture networks.

In Chapter 3, the indexing verification showed that the CVFE simulator can accurately represent displacement physics in DFN modeled fracture networks. In this chapter, an irregular basement reservoir model was used for upscaling studies. An absolute permeability upscaling technique (geometric Oda method) was examined for testing the transformation from the DFN fine-grid model to a coarse-grid flow model. A new, fine grid with a fracture intensity analysischaracterized fracture/nonfracture basement model was also tested by using ECLIPSE for the convergence with the CVFE simulation results. The upscaling procedure enables creation of single-porosity input files. The single-porosity simulations were performed using ECLIPSE. The grid resolutions in the single porosity simulations were critical in capturing the geologic information from the DFN models; however, in all cases a prespecified resolution did not produce the same result as the DFN approach. Instead, the prespecified resolution was dependent on fractured network geometry and the physical problem at hand. The advantage of using upscaled property is to speedup the simulation process. A portfolio of comparative simulation results helped us to better understand the level of uncertainty that might be introduced when using equivalent property and multiphase simulators to represent fractured reservoir systems. 


\section{Introduction}

For many years now, the most popular method of constructing detailed geological models of oil/gas reservoir was the classical deterministic method, which is widely applied in the fractured reservoir characterization. Geologists use this approach to interpret the existing data allowing them to build target-fractured reservoir models. However, since the past decade or so, deterministic approaches have been complemented by more and more quantitative geo-statistical approaches, such as multipoint statistics (MPS) (Horne et al., 2004, Liu et al., 2004) and discrete fracture network (DFN) (Dershowitz et al., 1998) modeling. Compared to the MPS model, the DFN model has the advantage of presenting fracture orientation explicitly. This allows the geologists to model the flow units and fracture flow paths.

Experience in fractured reservoir with low permeability formations often produces fluids through open fractures. An extreme case of Type I (Nelson's, 2001) classified reservoir will be considered in this study. In Type I fractured reservoir, fractures provide the essential reservoir porosity and permeability. Geologically, Type I reservoirs are granitic/basalt formations. They are also called "Basement Reservoirs." Practically all of the oil in these reservoirs resides in fractures or fault zones. Since the geometry of the fracture network is critical in understanding recovery from these reservoirs, these lend themselves well for treatment by using the DFN approach. The primary questions to be answered are:

1) Are DFN models accurate in modeling physics of displacement in these reservoirs?

2) How could DFN properties be homogenized into the conventional finite-difference simulators for the multiphase flow calculations?

3) For the process of "homogenization," how accurate can they be and how can multiphase model impact it? 
The first question was answered by indexing verification work in Chapter 3. The benchmark work showed that CVFE simulator can accurately represent the displacement physics of the fractured basement reservoirs.

Once the accuracy of the DFN approach was ascertained, an irregular basement network was constructed for the simulation purpose and the DFN approach was used directly to obtain simulation results. It was also possible to "homogenize" the irregular fracture domain and create "equivalent" ECLIPSE input files. This entails the creation of porosity and permeability fields, using one of the DFN upscaling methods (Oda method). The geometry-based Oda method was used to upscale permeability tensors initially defined in the discrete fracture network. Volumetric (P33) fracture intensity was calculated in each grid block to represent the upscaled porosity. Upscaling with a series of different grid block sizes (ranging from 10-ft to 200 -ft cubes) in a 1000 by 1000 by $200 \mathrm{ft}$ reservoir volume revealed that the upscaled results depend strongly on the relationship between grid block size, multiphase impact, fracture network geometry and simulator type. This study helped us evaluate the effectiveness of the upscaling techniques, and also compared the DFN approach to what can be considered a "single-porosity" simulation method toward the Type I basement reservoir.

\section{Simulating Fractured Reservoirs}

Conventional reservoir simulators use finite-difference methods with two-point flux calculations.

With the development of full tensor permeability (Lee et al., 1994) and nonorthogonal grid technique, multipoint flux calculations (Verma and Aziz, 1996; Gunasekera et al., 1998) are becoming more and more popular since the last decade. At the same time, control volume methods (Aavatsmark et al., 1997) have become the method of choice for today's simulator because of their capacity for handling complex geometric systems using unstructured grids. To 
achieve higher accuracy, limited work has also been done on advanced finite element methods (Young, 1978; Sukirman and Lewis, 1994). Yang (2003) developed an upstream transmissibility weighted control-volume finite-element discrete fracture network simulator.

Three types of main model formulations are popular for modeling and simulating flow through naturally fractured reservoirs: the single porosity model, the dual porosity model, and the DFN model. For detailed background regarding these three fracture simulation models refer to Section 2.3 .

\section{Proposed Domain Characteristics}

Common model properties for both domains:

- Impermeable matrix with $\Phi=0$ (Type I, basement reservoir system)

- $\quad$ Domain $=1,000 \mathrm{ft}$ by $1,000 \mathrm{ft}$ by 200 feet deep

- Total feature length $=30,000$ feet

- Feature property: $k=1,000 \mathrm{md}, \Phi=14 \%$, width $=0.5$ feet

- $\quad$ Original Oil In Place (OOIP) $=53,580$ STB

- Injection Pressure $=4,300 \mathrm{psi}$

- $\quad$ Lower dot $=$ Injection Well, upper dot $=$ Production Well

As shown in Figure 4.1, the regularized and irregularized fracture network domains were generated. Two constraints were applied for the DFN irregularized domain and its equivalent regularized domain: the same OOIP and the same transmissivity (kh value). Both domains were characterized as a three-phase black-oil model with initial solution gas in the oil phase and the exact same fluid and rock properties. Examples of these properties include fluid density, viscosity, compressibility, PVT table, formation factor, gas/oil ratio table, rock compressibilities, 
relative permeability curves, initial conditions, well operation conditions and schedules, etc. For detailed geological information, refer to Section 3.4.

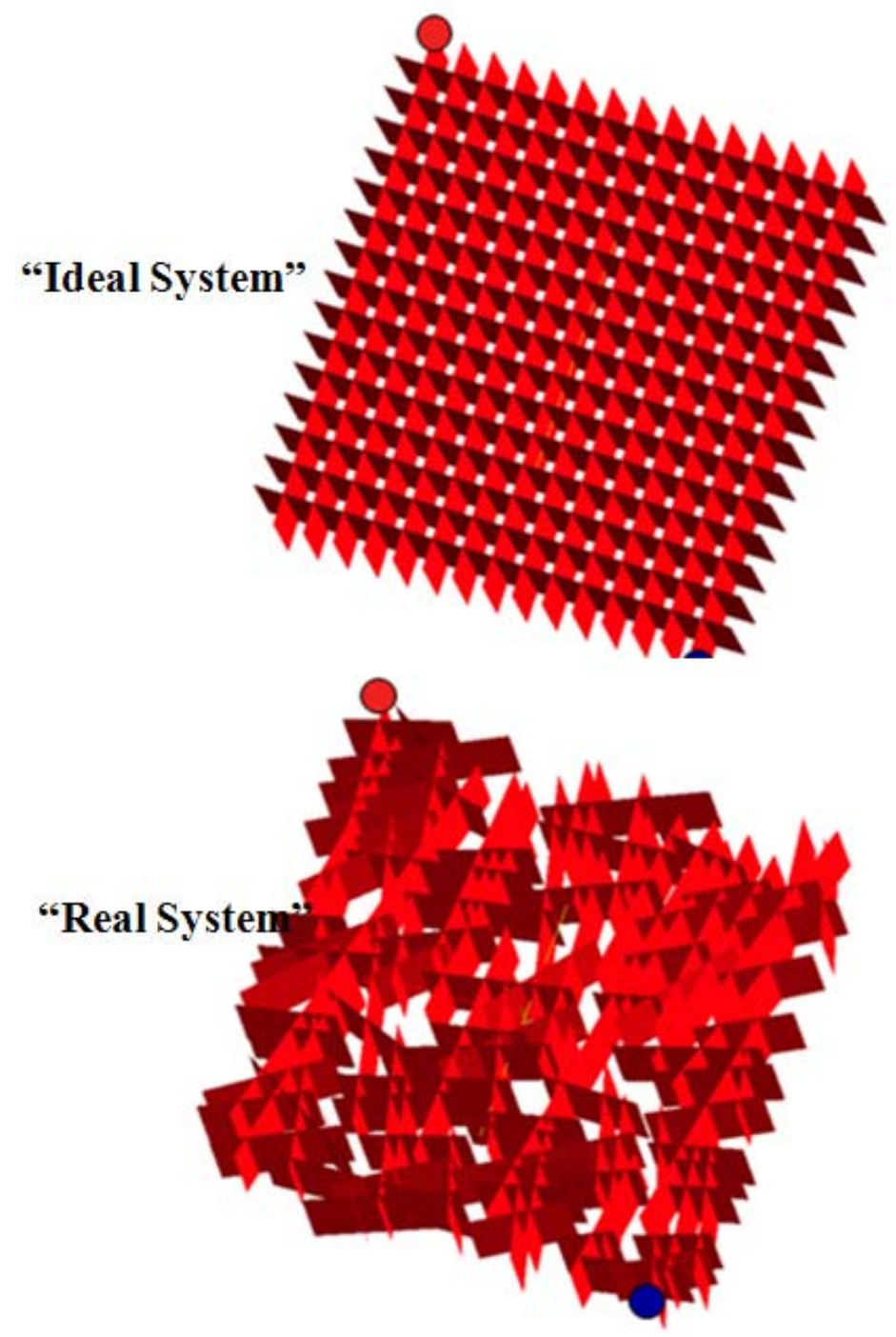

Figure 4.1 Irregular "real" DFN basement model (bottom) and equivalent regularized "ideal" DFN basement model (top)

\section{CVFE Simulator Verification}

As mentioned at the beginning of this chapter, the CVFE simulator has been indexed verified by with the well-known commercial reservoir simulator ECLIPSE in Chapter 3. Some other CVFE 
formulation verifications have been reported through the manufacture solution method (Yang, 2003; Fu, 2007).

\section{Study of Hypothetical “Real” Basement Reservoir Model}

In this section, the hypothetical "real" basement system was studied using the CVFE simulator as the base solution of series of upscaling/integrating approaches. For both "ideal" and "real" systems, the analyses of fracture/fault orientation and connectivity are discussed in Section 3.4. Other than that, "real" and "ideal" basement systems shared all of the remaining reservoir characteristics and operating information, including the well positions. From this point of view, this study is trying to answer the following questions:
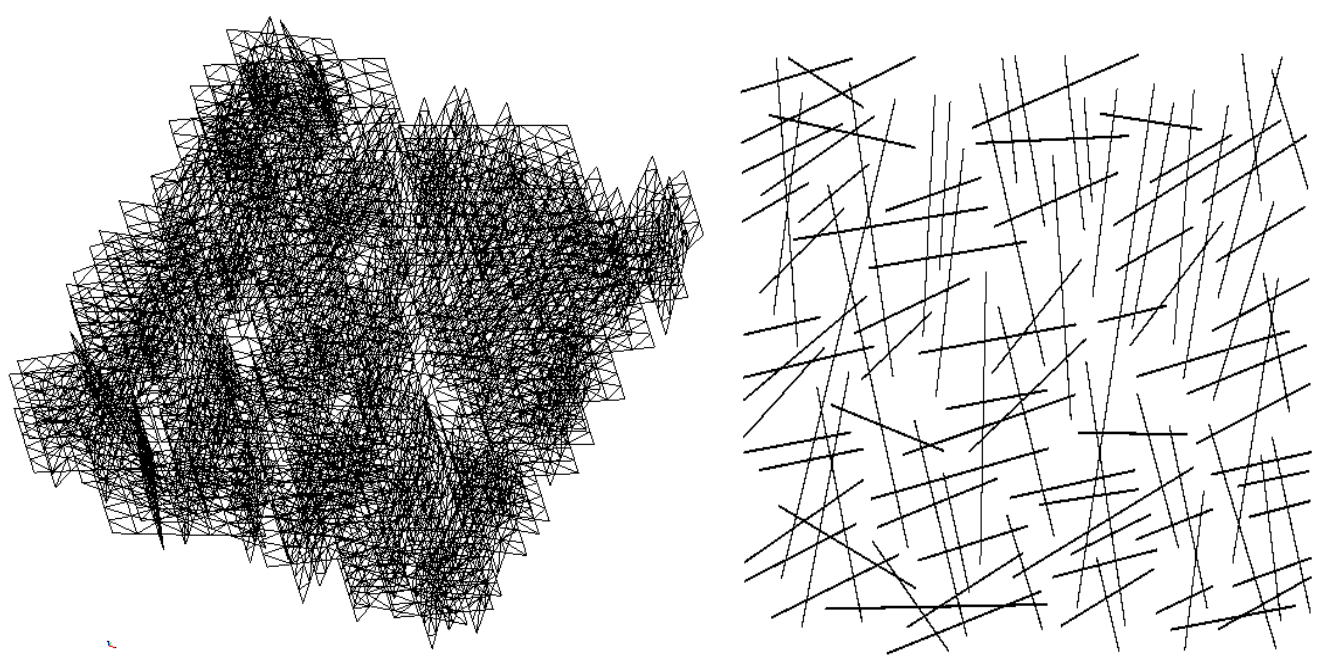

Figure 4.2 Three-dimensional view (left) and two-dimensional view (right) of discretized, irregularized DFN modeled "real" basement reservoir domain for the CVFE simulator

- Can a "real" basement reservoir be presented by an "ideal" system regardless of fracture/fault orientation and connectivity? (Figure 4.2) 
- Can a "real" basement system be represented (upscaled or integrated) by the conventional finite-difference black-oil simulator?

- If the basement system could be studied by ECLIPSE, will grid-block size impact the oil recovery results?

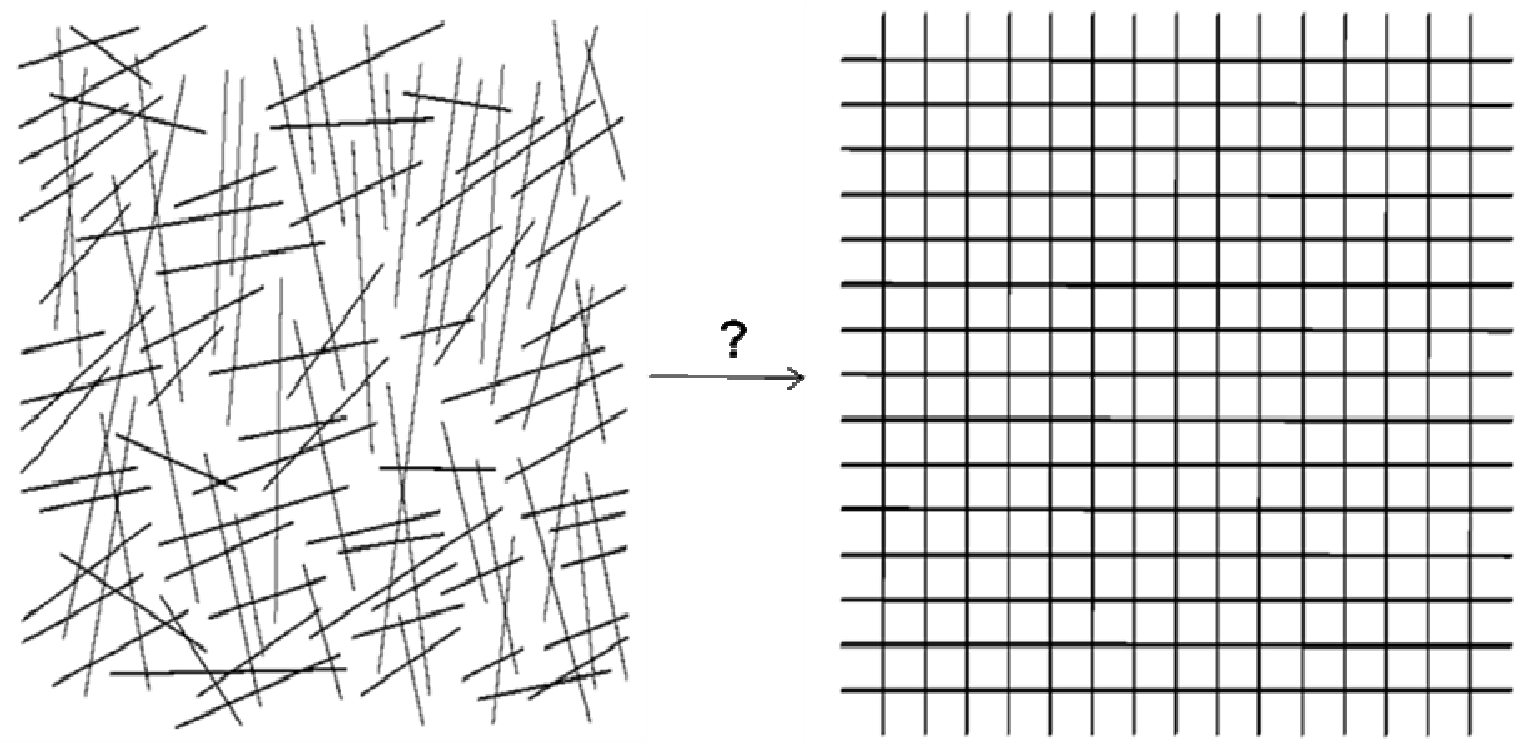

Figure 4.3 Simplification of "real" basement system (left) to "ideal" basement system (right)

\section{Effect of Fracture/Fault Orientation and Connectivity}

From the fracture cluster analysis, the "real" basement fracture/fault system was considered the "well connected" system. One of the hypotheses regarding this "well connected" system was the question of whether this system could be simplified as the "ideal" system. (Figure 4.3) During this simplification process, 107 fractures are treated as 30 uni-sizes perfectly connected fracture network. Other than that everything stays the same except the connectivity and orientation. To answer this question, a simulation was performed on the "real" basement system. 
Figures 4.4 through 4.6 show that on the stage of primary production, the "ideal" system and the "real" system are well matched. The only driving force on this production stage is the reservoir pressure. At the end of primary production, the reservoir pressure is dropped to $2370 \mathrm{psi}$ which is lowered than the fluid model bubble point at 2700 psi.

Figure 4.7 shows the gas phase saturation distributions under these conditions. Since there is no isolated fracture in both "real" and "ideal" systems, much of the "mobilized" gas-phase is evenly distributed on the top of the reservoir. This even gas distribution made the system pressure evenly distributed and explained the excellent match during primary production.

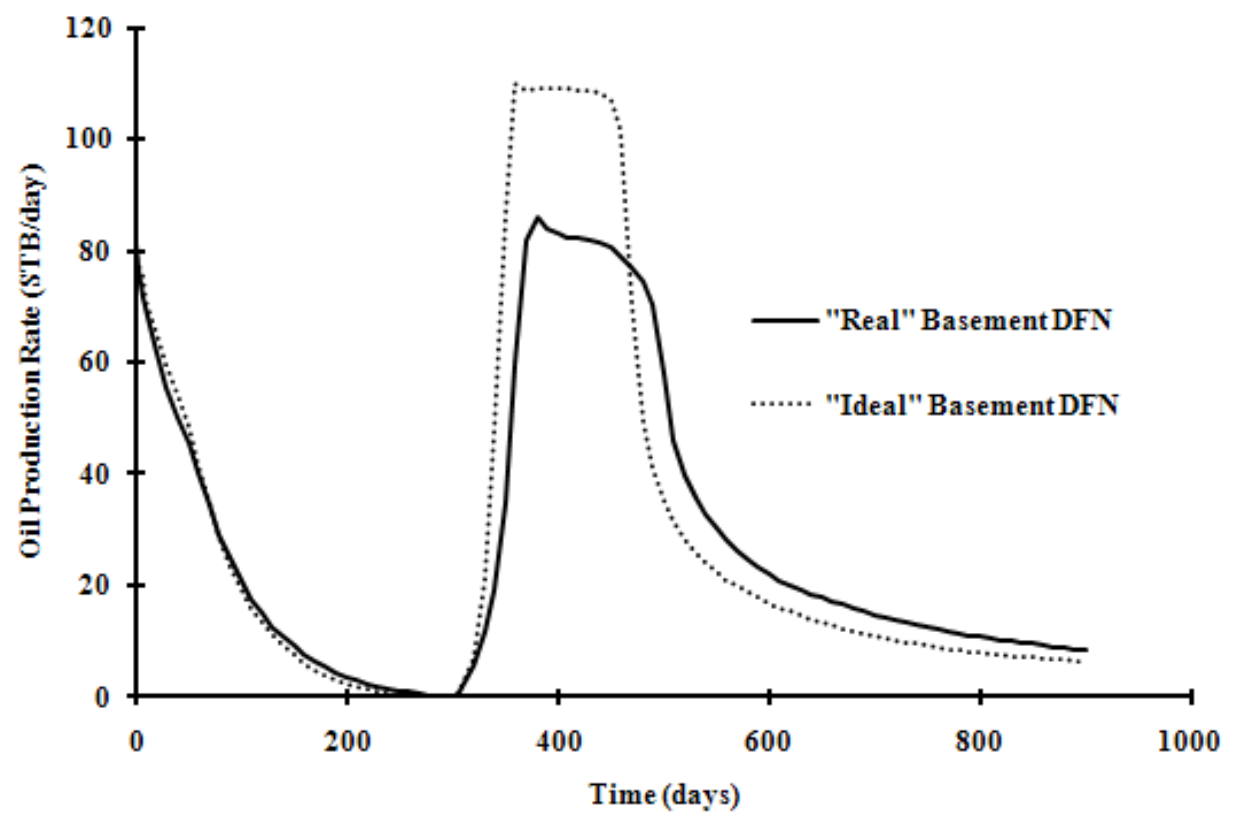

Figure 4.4 Comparisons of oil production rate between the "ideal" and "real" system 


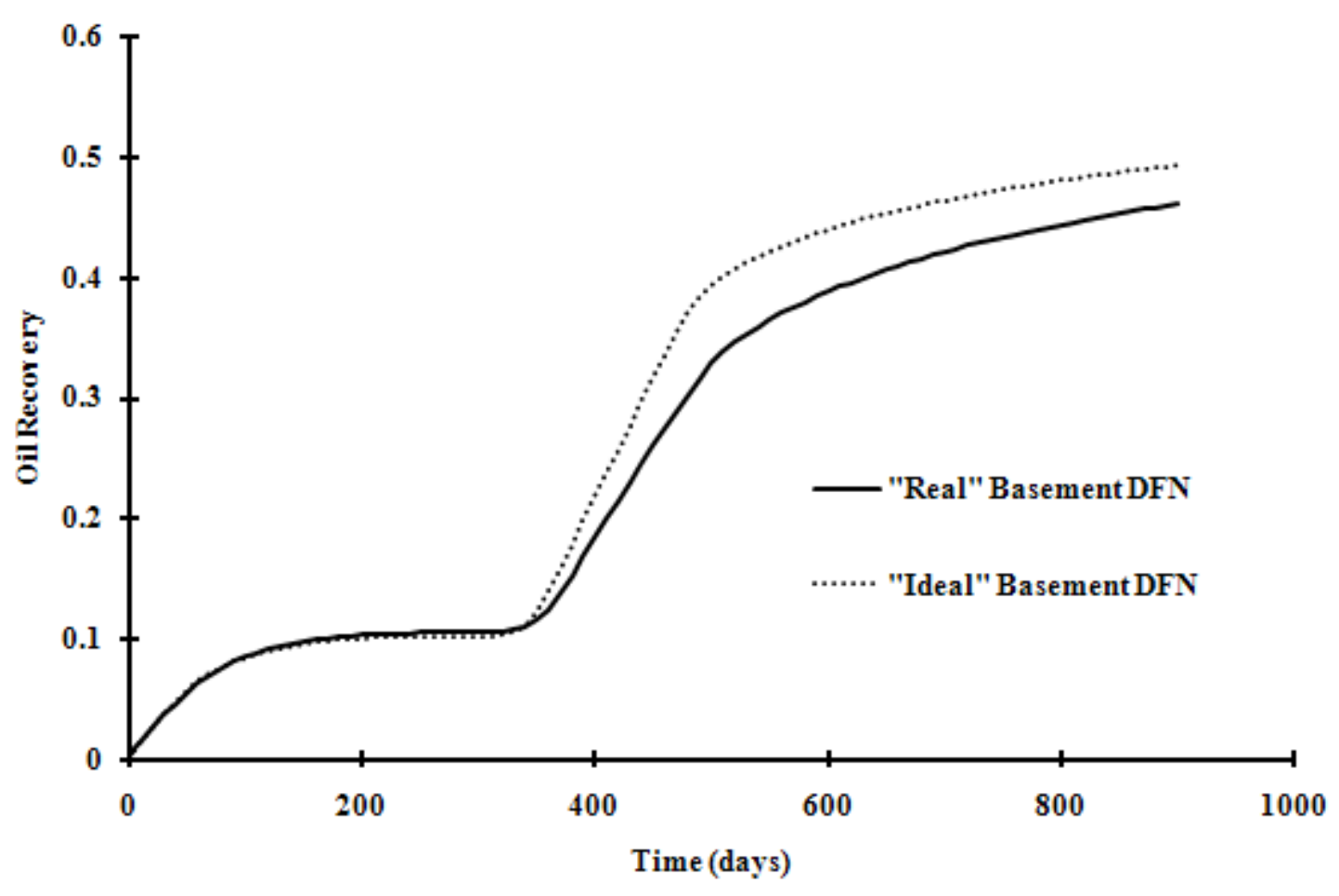

Figure 4.5 Dimensionless analysis of cumulative oil production rate between the "ideal" and "real" systems
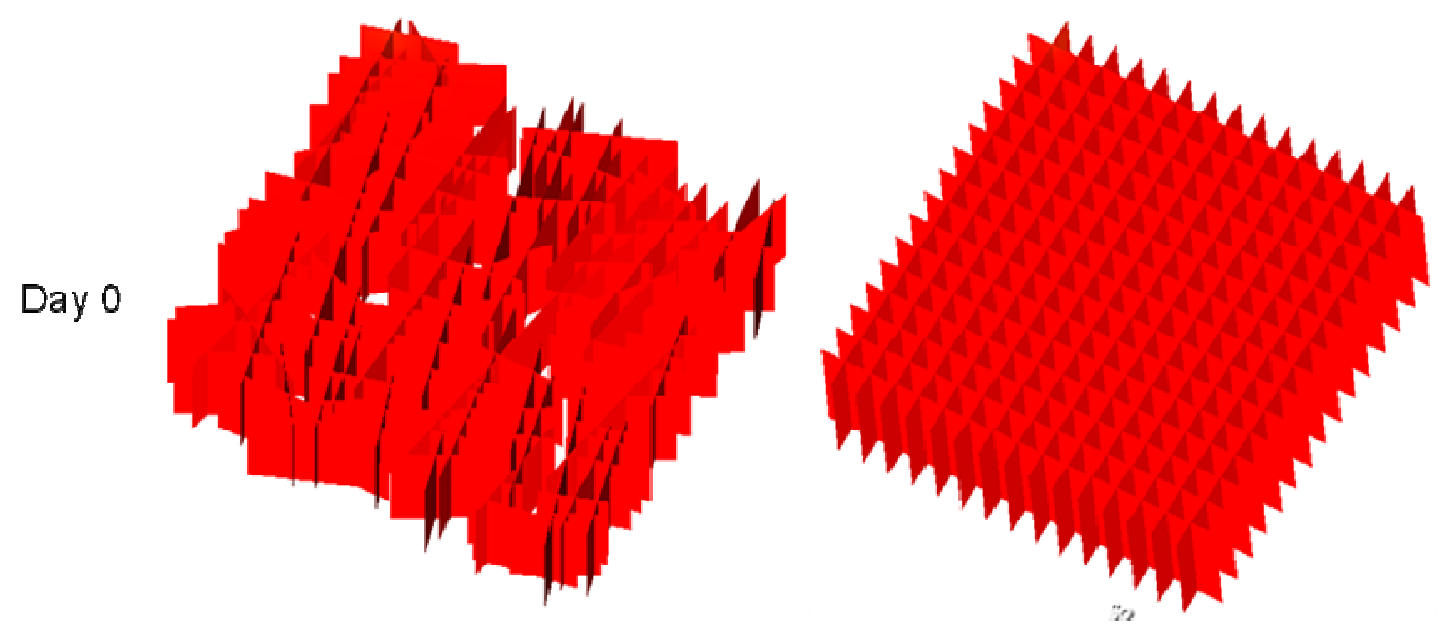

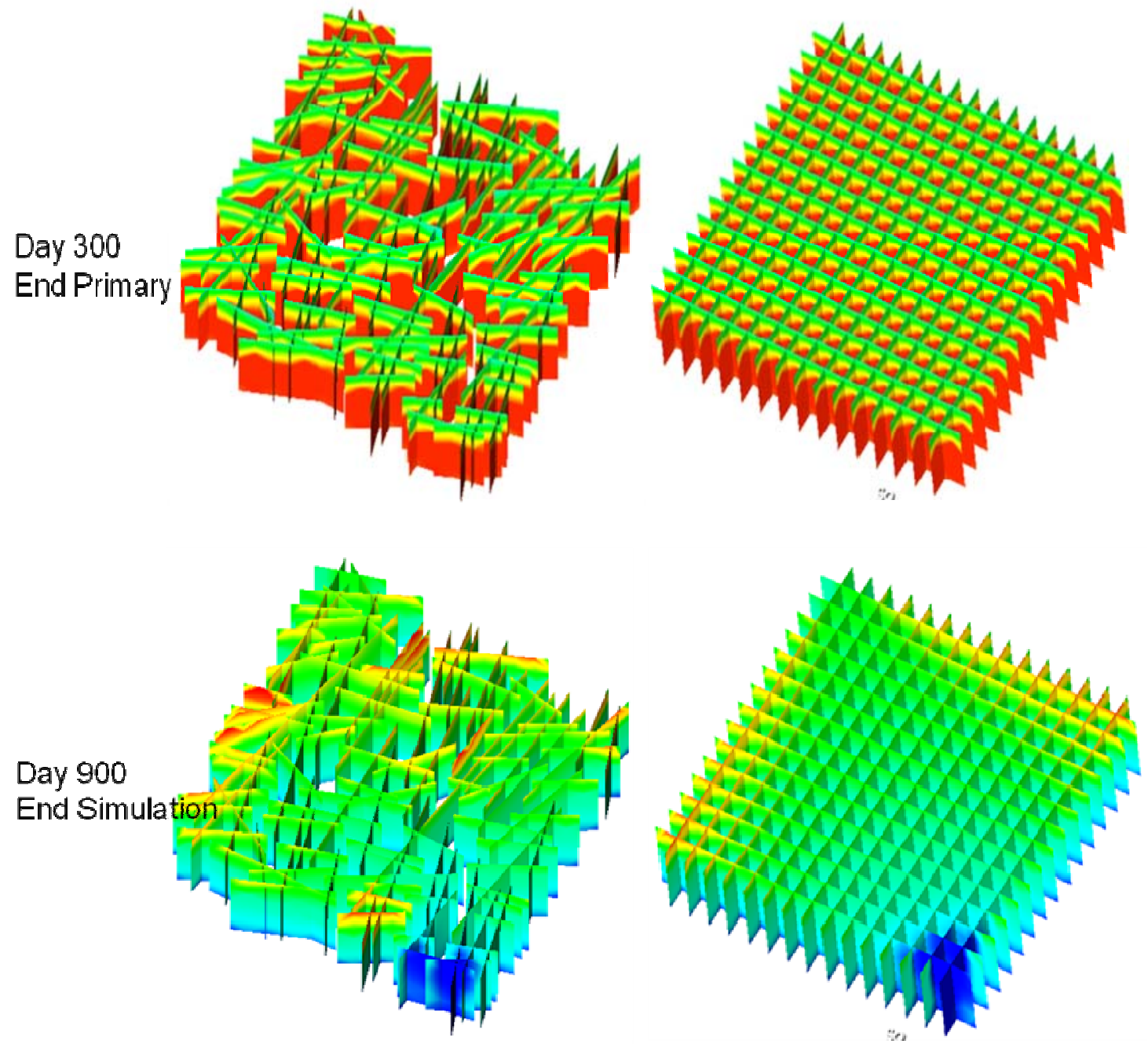

Figure 4.6 Snapshot of oil saturations for the "real" basement system and "ideal" basement system

In secondary production, the significant differences are shown between "real" and "ideal" systems. Quantitatively, the peak daily oil production rate from the "ideal" basement system was $27.13 \%$ higher than the "real" basement system, and the cumulative oil production from the "ideal" system was $6.77 \%$ higher than from the "real" system at the end of simulation. Instead of an even water flooding pattern from the "ideal" system, the oil distribution snapshot in Figure 4.6 shows that the "real" system has more stranded oil spots than the "ideal" system. Obviously, these oil trappings are a result of the fracture/fault connectivity. An interesting observation found at the end of simulations is that the "ideal" oil production rate is only slightly higher than "real" 
production rate. This is due to the similar reservoir driving force at the day 900 ("real": 2870 4130 psi; "ideal": $2940 \sim 4100$ psi). At the end of the simulations, the "ideal" system shows a little narrower pressure distribution than the "real" system due to the local fracture/fault cluster connectivity issue.

Figure 4.8 shows that the fracture/fault connectivity plays an important role in the basement reservoir study. In the "real" system, the pressure has a significant drawn-down near the main connections between the injector and the producer. This phenomenon can be found from the "ideal" system oil pressure distributions.
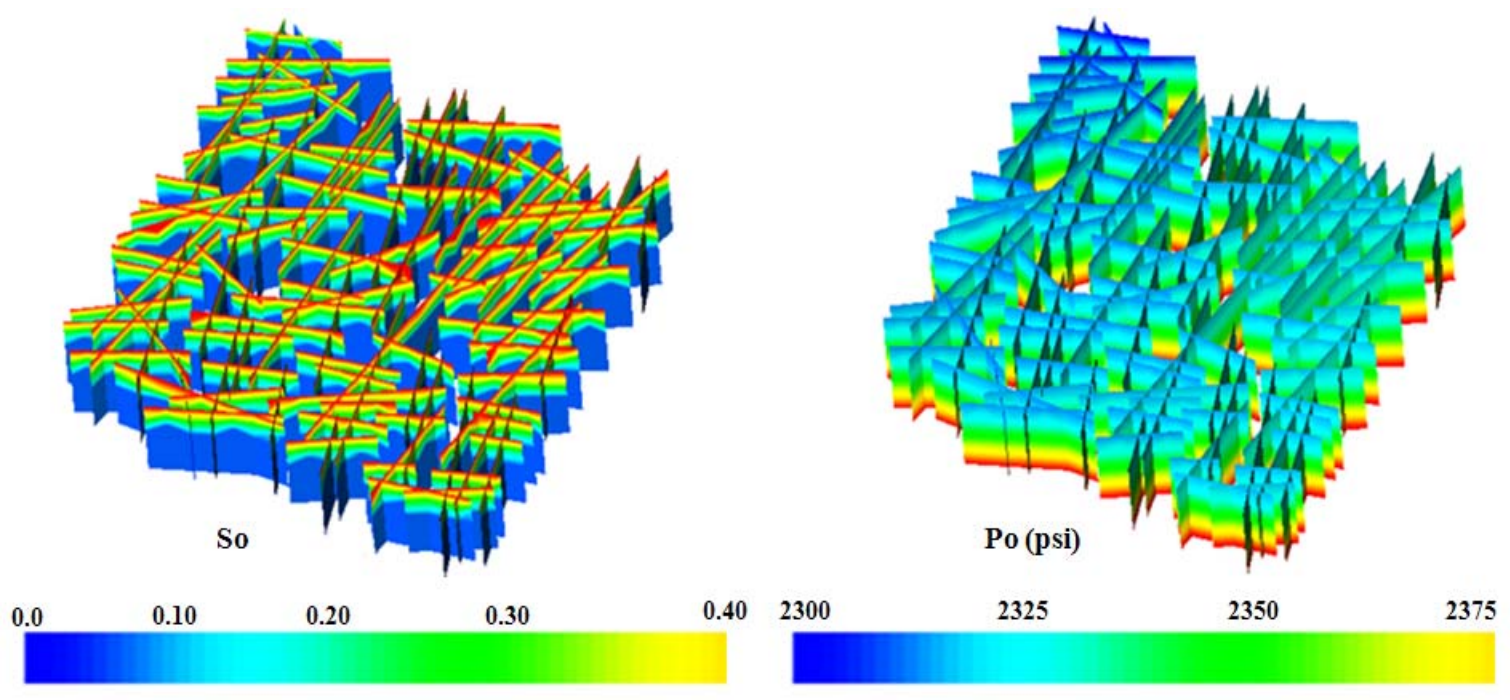

Figure 4.7 At day 300 (end of set primary production), the gas saturation (left) and the oil pressure distributions (right) 


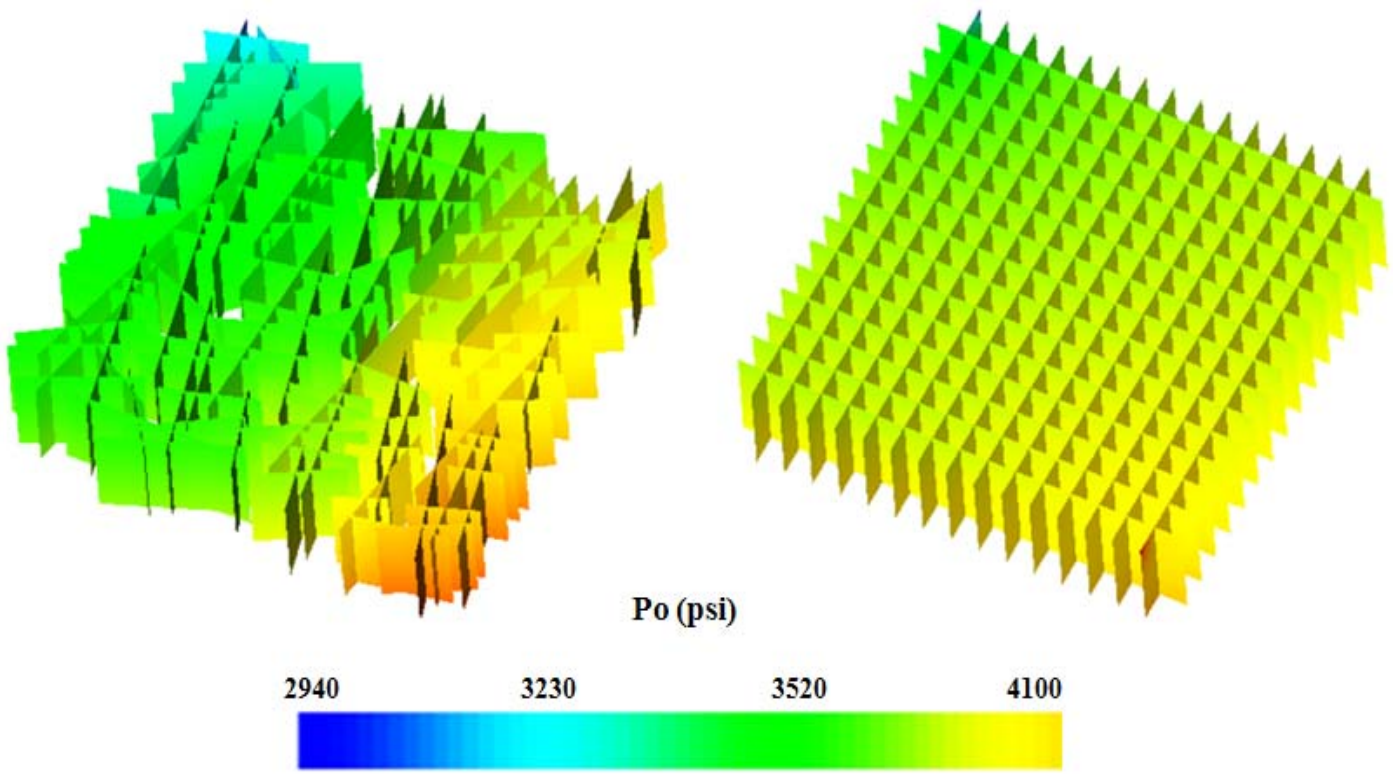

Figure 4.8 At the end of simulation (day 900), the pressure distributions on "real" (left) basement model and the "ideal" (right) basement model

This study suggests the following conclusions:

1) The fracture/fault cluster connectivity plays an important role in fractured reservoir productions.

2) In primary production stage, the "real" system can be represented by the regularized “ideal" system.

3) In the secondary production scenario:

a. Theoretically, the "real" system cannot be described precisely by the equivalent "ideal" system for the water flooding study.

b. Practically, the "ideal" system can be used for the fast oil production forecast qualitatively. However, this might be too risky due to the existence of many uncertainties. 
4) CVFE simulator is a powerful tool for studying DFN modeled fractured reservoirs. The "real" fracture/fault model is difficult to describe and calculate through conventional finite-difference based reservoir simulators.

\section{Representation of DFN Modeled Basement Fracture Network Using a Finite Difference Based Simulator}

Reservoir simulation can be significantly more challenging for naturally fractured reservoirs than it is for conventional classic reservoirs. In the Type I basement reservoir, all hydrocarbons are stored and flow among the fractures/faults network. Since most modern reservoir simulators are based on finite-difference method, it becomes interesting to ask whether the DFN modeled fracture network can be simulated by a conventional finite-difference simulator such as ECLIPSE. If the answer were "yes," the accuracy and stability of this integration would need to be quantified.

Dershowitz et al. (2000) first presented the method of integration of DFN with conventional simulator approach. In their work, the DFN model was characterized as having the fracture continuum for a single-phase dual porosity simulation. No multiphase integration work has been reported since then.

In this study, a DFN modeled "real" basement fracture network was "homogenized" or "equivalent" as the single porosity continuum model. This concept consists of overlapping the fracture/fault network and a finite-difference orthogonal grid, by considering the fracture properties such as density, permeability, thickness and porosity in each grid and assigning equivalent values to each grid cell in the finite-difference grid. Figure 4.9 shows how this concept works. 
As shown in Figure 4.9, the basement reservoir properties are "homogenized" by the DFN model into the finite-difference grid blocks. For flow simulations, homogenized grid porosity and permeability are discussed in the following sections.

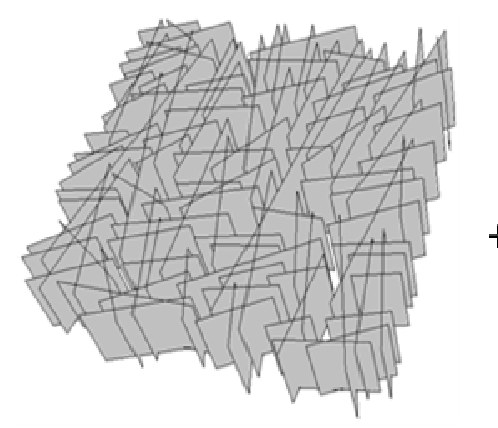

Fracture Network

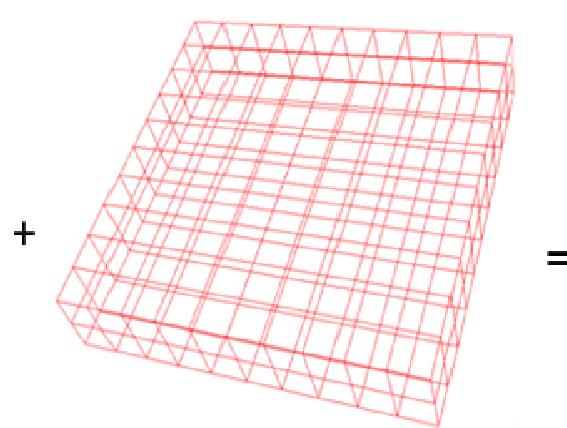

Gridblocks

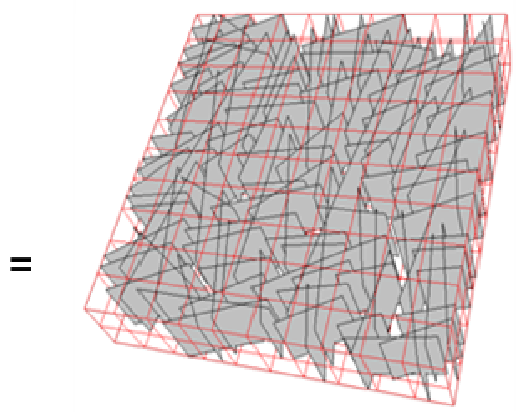

Equivalent Single Porosity

Figure 4.9 "Homogenized" or "Equivalent" single porosity modeled "real" basement reservoirs

\section{Grid Block Porosity}

The fracture-system porosity was calculated as the product of the fracture intensity expressed as fracture area per unit volume (P32) and the storage thickness of fracture/fault (Equation (4-1)):

$\Phi_{\text {GridBlock }}=\frac{V_{D F N}}{V_{\text {GridBlock }}}=\frac{\sum\left(A_{D F N} \cdot e\right)}{V_{\text {GridBlock }}}=P_{32} \cdot e$

where:

$\Phi_{\text {GridBlock }} \quad=\quad$ basement fracture/fault porosity

$V_{D F N} \quad=\quad$ basement fracture/fault volume

$V_{\text {GridBlock }}=$ finite-difference grid block volume

$A_{D F N} \quad=\quad$ basement fracture/fault area

$e \quad=\quad$ basement fracture/fault storage thickness

$\mathrm{P}_{32}=$ basement fracture/fault intensity as fracture area per unit volume 
To calculate the "equivalent" porosity for each grid cell, the fracture/fault volume needs to be averaged by the volume of the grid blocks.

\section{Grid Block Permeability}

The approaches for calculating continuum grid block effective directional permeability can be achieved by two main methods: 1) the geometry-based Oda method (Oda, 1985), and 2) the flow-based "Block K" method. These two methods are reviewed below.

In a three-dimensional system, single-phase fluid flow through an isotropic porous media is described by the differential form of Darcy's law (Equation (4-2)):

$\mathbf{q}=-\frac{\underline{\mu}}{\mu} k \nabla \mathbf{P}\left(\mathrm{x}_{y} y_{r} z_{z}\right)$

where

$\nabla \mathrm{P}(\mathrm{x}, \mathrm{y}, \mathrm{z})=\left(\frac{\partial \mathrm{P}}{\partial_{x}}, \frac{\partial_{\mathrm{P}}}{\partial_{\mathrm{Y}}}, \frac{\partial_{\mathrm{P}}}{\partial_{\mathrm{z}}}\right)$

is the pressure gradient at the point $(\mathrm{x}, \mathrm{y}, \mathrm{z})$, and $\mathbf{q}$ is the resultant flux vector (mass per unit time per unit area) at the point. The isotropic permeability $\mathrm{k}$ is a local property of the medium, while the density, $\rho$, and viscosity, $\mu$, are properties of the fluid.

A $3 \times 3$ symmetric permeability tensor of a fracture could be calculated by projecting its isotropic permeability, $\mathrm{k}$, onto its plane (Oda's method). Let $\mathrm{n}$ denote the unit pole (normal) vector of the fracture. The components of the Oda tensor can be derived by subtracting the normal component of the flux, leaving a flux vector $\mathrm{q}_{\mathrm{f}}$ in the plane of the fracture, as shown in Equation (4-4):

$$
\mathbf{q}_{f}-\mathbf{q}-\mathbf{n} \mathbf{n}^{\mathrm{T}} \mathbf{q}-\left(\mathbf{I}-\mathbf{n} \mathbf{n}^{\mathrm{T}}\right)-\mathbf{q}
$$


where $\mathbf{I}$ is the $3 \times 3$ identity matrix, and $\mathbf{n n}^{\mathrm{T}}$ is the matrix outer product given by Equation (4-5):

$$
\mathbf{n n}^{T}=\left[\begin{array}{ccc}
n_{1}^{2} & n_{1} n_{2} & n_{1} n_{a} \\
n_{1} n_{2} & n_{2}^{2} & n_{2} n_{2} \\
n_{1} n_{a} & n_{2} n_{2} & n_{a}^{2}
\end{array}\right]
$$

Substituting these expressions back into Darcy's equation, and accounting for the ratio between the fracture volume (thickness, e, multiplied by area, A) and the cell volume, V, Equation (4-6) is defined as:

$$
\mathbf{q}_{\mathrm{f}}=-\frac{\rho \mathrm{keA}}{\psi \mathrm{v}}\left(\mathbf{I}-\mathbf{n} \mathbf{n}^{\mathrm{T}}\right) \nabla \mathbf{P}=-\frac{\rho}{\mu} k \nabla \mathbf{P}
$$

where the six unique entries of the symmetric Oda tensor $\mathbf{K}$ are simply represented by Equation (4-7):

$k_{j}=\mathbf{k} \frac{E A}{\vartheta}\left(g_{j, j}-n_{j} n_{j}\right)$

The indices I and $\mathrm{j}$ can be 1, 2, or 3 (for each coordinate direction), and the Kronecker delta function is given by Equation (4-8):

$$
\delta_{i 1}=\left\{\begin{array}{l}
1, \text { when } 1=1 \\
0, \text { othorweo }
\end{array}\right.
$$

If the flux through each fracture within the cell is assumed to be additive, then these matrices can be summed together to get a net permeability tensor $\mathbf{K}_{\text {net }}$. The principal permeabilities and flow directions within this cell are given by the eigen values and eigenvectors of $\mathbf{K}_{\text {net }}$. 
One drawback of Oda's method is that it does not take into account fracture network connectivity and therefore is limited to well-connected fracture networks. The "real" basement model is a well-connected fracture network.

The second approach of equivalent directional permeability tensor calculations could be done by the "block-K" method (Long, 1984; Doolin et al., 1995 and Clemo, 1998).

Figure 4.10 shows how $\mathrm{x}$-directional permeability tensors are calculated. Starting with areal map of a "real" basement DFN model and overlapping with a 10x10x2

orthogonal finite-difference grid block, a single-phase (for example, water) steady-state flow along x-direction has been applied on the circled grid block cell. The only pressure gradient is in the x-direction (as shown in Equation (4-9).

$\nabla P=\frac{\partial P}{\partial X} \hat{i}$

The Darcy equation could be simplified in Equation (4-10) :

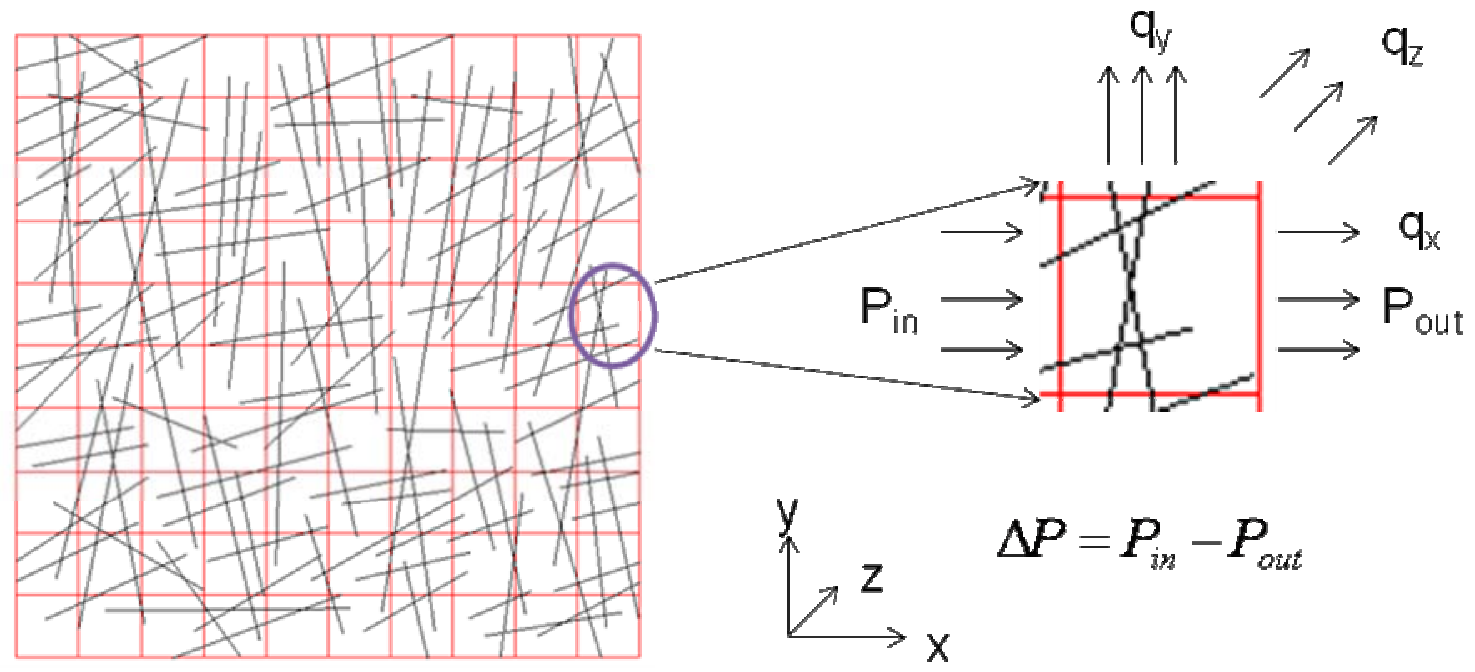

Figure 4.10 Illustration of the block-K method for calculating the $\mathrm{x}$-directional permeability tensor 
$\left[\begin{array}{l}q_{x} \\ q_{y} \\ q_{z}\end{array}\right]=\left[\begin{array}{lll}k_{x x} & k_{x y} & k_{x z} \\ k_{y x} & k_{y y} & k_{y z} \\ k_{z x} & k_{z y} & k_{z z}\end{array}\right]\left[\begin{array}{c}\frac{\partial P}{\partial x} \\ 0 \\ 0\end{array}\right]$

The above equations could be represented by Equations (4-11) to (4-13)

$q_{x}=k_{x x}\left(\frac{\partial P}{\partial x}\right)$

$q_{y}=k_{x y}\left(\frac{\partial P}{\partial x}\right)$

$q_{z}=k_{x z}\left(\frac{\partial P}{\partial x}\right)$

The directional permeability tensor could be calculated by inverting above three equations to Equations (4-14) through (4-16):

$k_{x x}=\frac{q_{x}}{\left(\frac{\partial P}{\partial x}\right)}$

$k_{x y}=\frac{q_{y}}{\left(\frac{\partial P}{\partial x}\right)}$

$k_{x z}=\frac{q_{z}}{\left(\frac{\partial P}{\partial x}\right)}$

The block-K method is based on the steady-state flow simulation on each separated grid block cell. Compared with Oda's method, it has some drawbacks such as numerical tolerance of block cell face curvatures and the computation times. In the study, only Oda's method will be applied since the fractures/faults are well-connected. 


\section{Case Studies on Simulating a "Homogenized DFN Basement" Continuum Single Porosity Model by Conventional Finite-Difference Simulator ECLIPSE}

The process of integrating a DFN modeled fracture network with conventional continuum grid blocks was discussed in the last section. This makes simulation of the DFN characterized fracture network possible through the conventional finite-difference simulator. In this section, various homogenized "real" DFN modeled fractured networks were simulated by ECLIPSE under multiphase conditions. Then the results from ECLIPSE were compared with the results from the CVFE simulator. These comparisons aided in understanding the accuracy and stability of this homogenization process if the results from CVFE simulator are presumed to be correct.

\section{Grid Block Sensitivity Studies with Both Porosity and Permeability Homogenization}

In this section, both porosity and permeability homogenization are studied regarding grid block size sensitivity. A series of simulations are designed with different grid block sizes (from 10-ft cube to a $333 \times 333 \times 200-f t$ grid block cell), as shown in Figure 4.11. For different grid block sizes, the porosity of the homogenized grid blocks are calculated and visualized as shown in Figure 4.12 .

Figure 4.12 shows that with various resolutions of grid block sizes, the local fracture/fault porosity density distributions are better honored by finer grid blocks. Examples of 3x3x1 grid block resolution resulted in close porosities being calculated other than 50x50x1 resolution which had distinguished porosities in each grid block. 

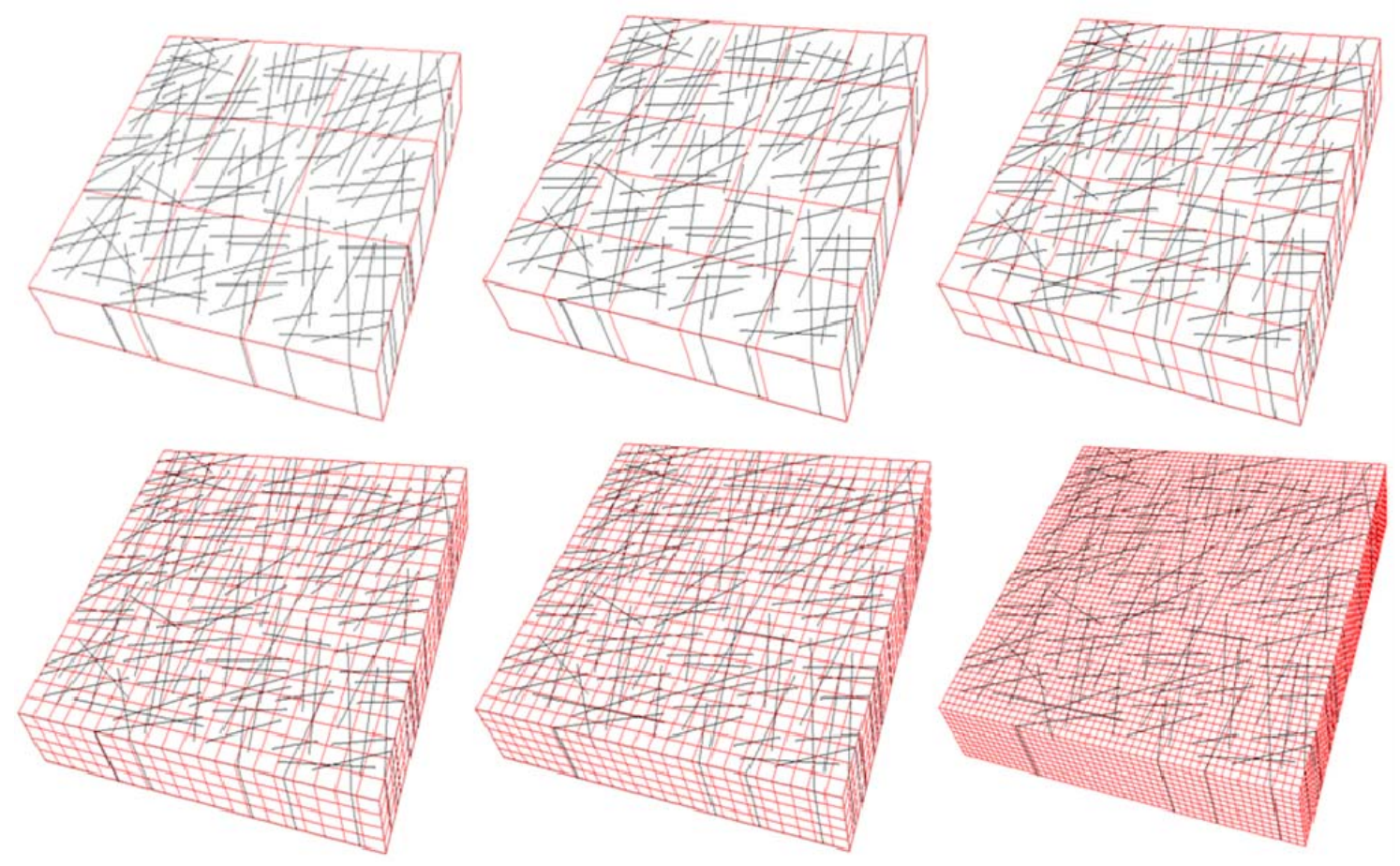

Figure 4.11 Illustrations of homogenized DFN grid with different cell sizes
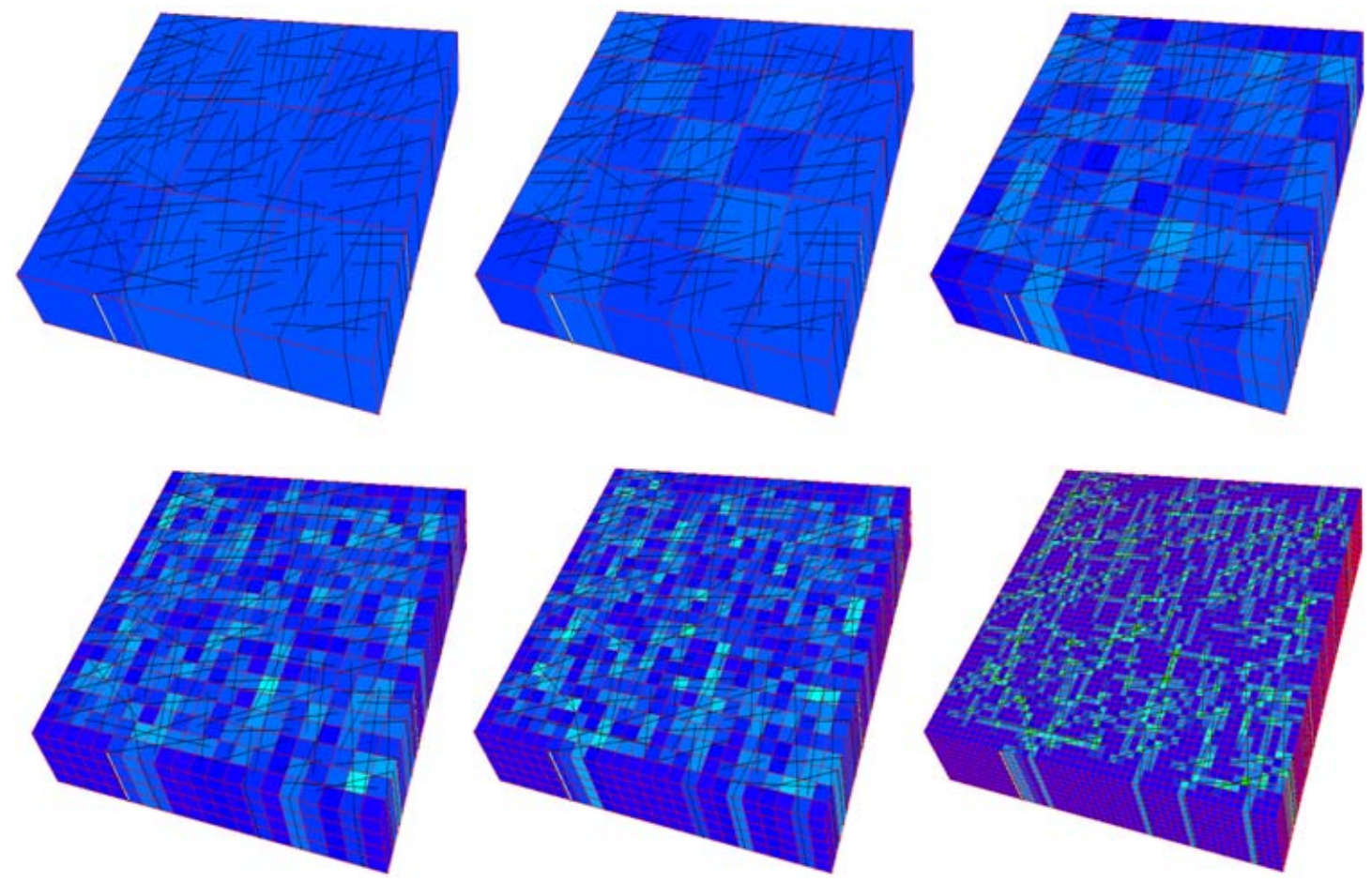

Figure 4.12 Visualization of porosity on homogenized DFN grids 
An example of permeability homogenization with the grid block size of $40-\mathrm{ft}$ cubes $(25 \times 25 \times 5$ grid blocks) is shown in Figure 4.13. The equivalent permeability tensor being calculated from Oda's method are a symmetric tensor, which means $k_{x y}=k_{y x}, k_{x z}=k_{z x}$, and $k_{y z}=k_{z y}$. Since all fractures in the "real" basement domain are vertical, the cross permeability term related to zdirection is zero, as the last two pictures shown in Figure 4.13.

A series of three-phase simulations with the same operating conditions were performed on the ECLIPSE simulator. Figure 4.14 shows that the solutions of oil production rates on the homogenized grid are sensitive to the grid block sizes. Starting from the coarsest grid $(3 \times 3 \times 1)$ blocks, and then proceeding to the more refined grid blocks, at the stage of primary production the finer grid presented better matches of CVFE solution. At the stage of secondary production, the reservoir oil production behavior is quite different from refined grid block sizes: Figure 4.13 shows that with refining grid block sizes, the oil production rate approaches the CVFE solution. Then the production rate passes the CVFE solution and gets further and further with the grid refinement. The finest grid in Figure 4.14 is the $40-\mathrm{ft}$ cube; some finer grids such as 20 -ft and 10 $\mathrm{ft}$ cubes were also studied but they cannot be converged. 

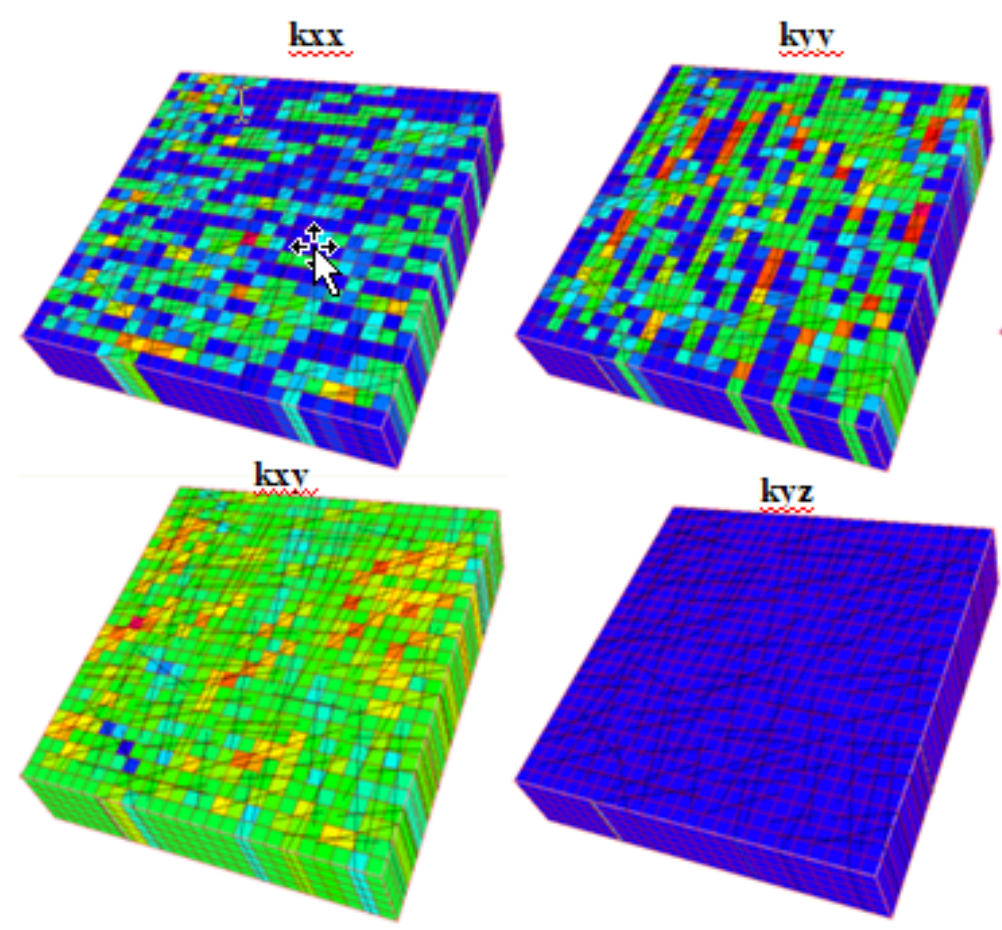

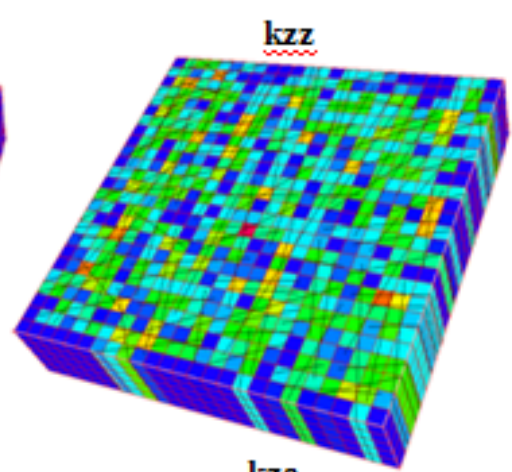

kza

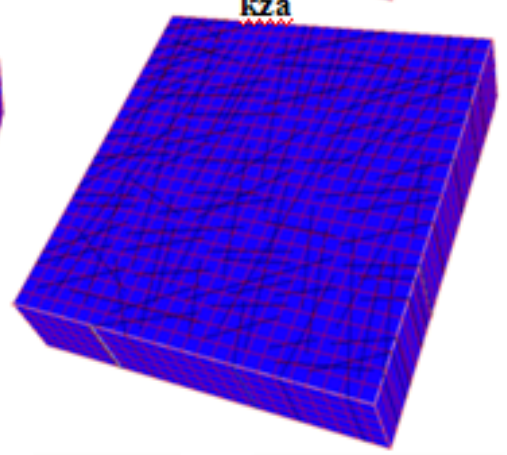

Figure 4.13 Visualization of the permeability tensors on homogenized DFN grids (The grid block sizes chosen are the $40-\mathrm{ft}$ cube, $25 \times 25 \times 5$ equal blocks on a $1000 \mathrm{ft} \times 1000 \mathrm{ft} \times 200 \mathrm{ft}$ domain.)

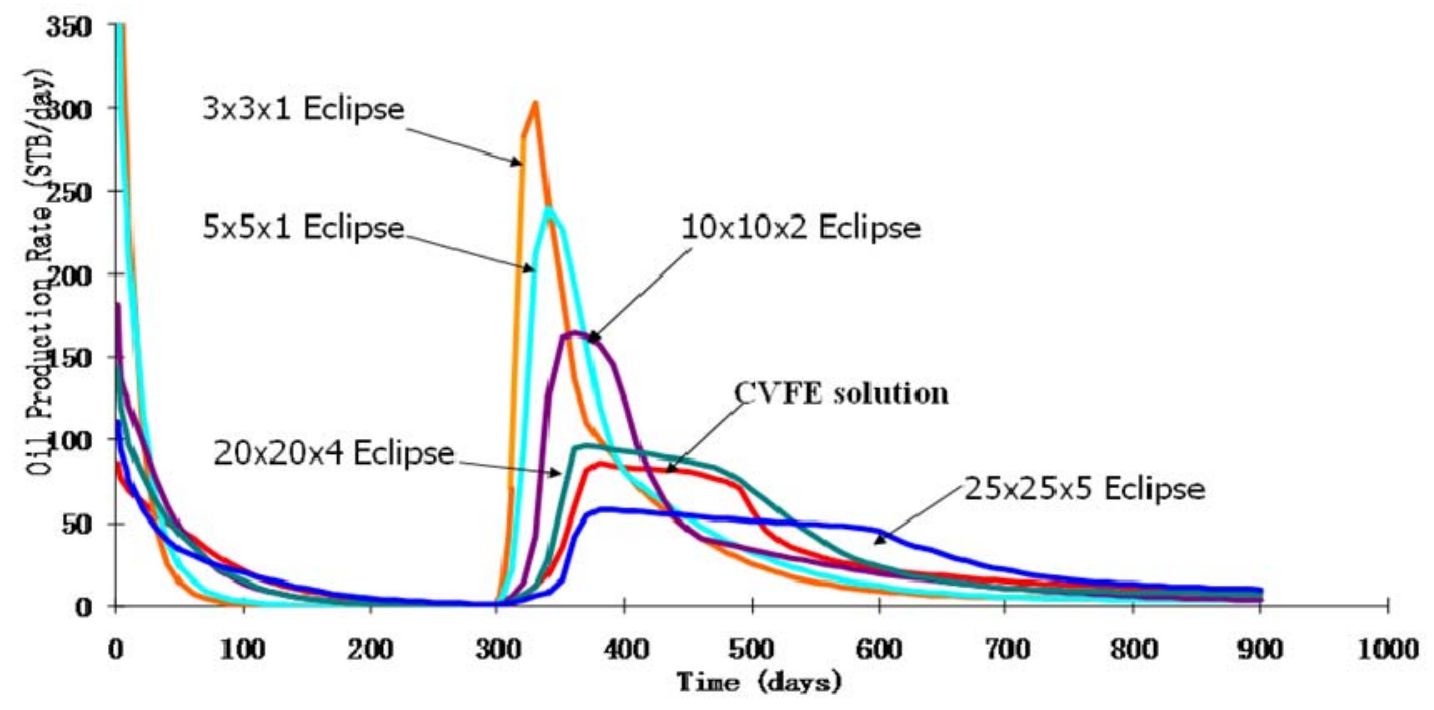

Figure 4.14 Oil production rate comparisons among homogenized ECLIPSE simulations and CVFE simulation 
Since the secondary production is the water flooding study, the water cut curve comparisons are shown in Figure 4.15.The same trends are discovered on the water cut curves among the various homogenized grid block sizes as compared to the CVFE solution. Meanwhile, in the case of presenting the "real" basement system, the homogenized 20x20x4 (50-ft cube) ECLIPSE simulation shows the best water breakthrough time match but still has certain errors after the middle of injection. The same phenomenon from the water cut figure is observed as from the oil production rates figure: the finer grids with both porosity and permeability homogenized are difficult to converge. The reason is discussed below.

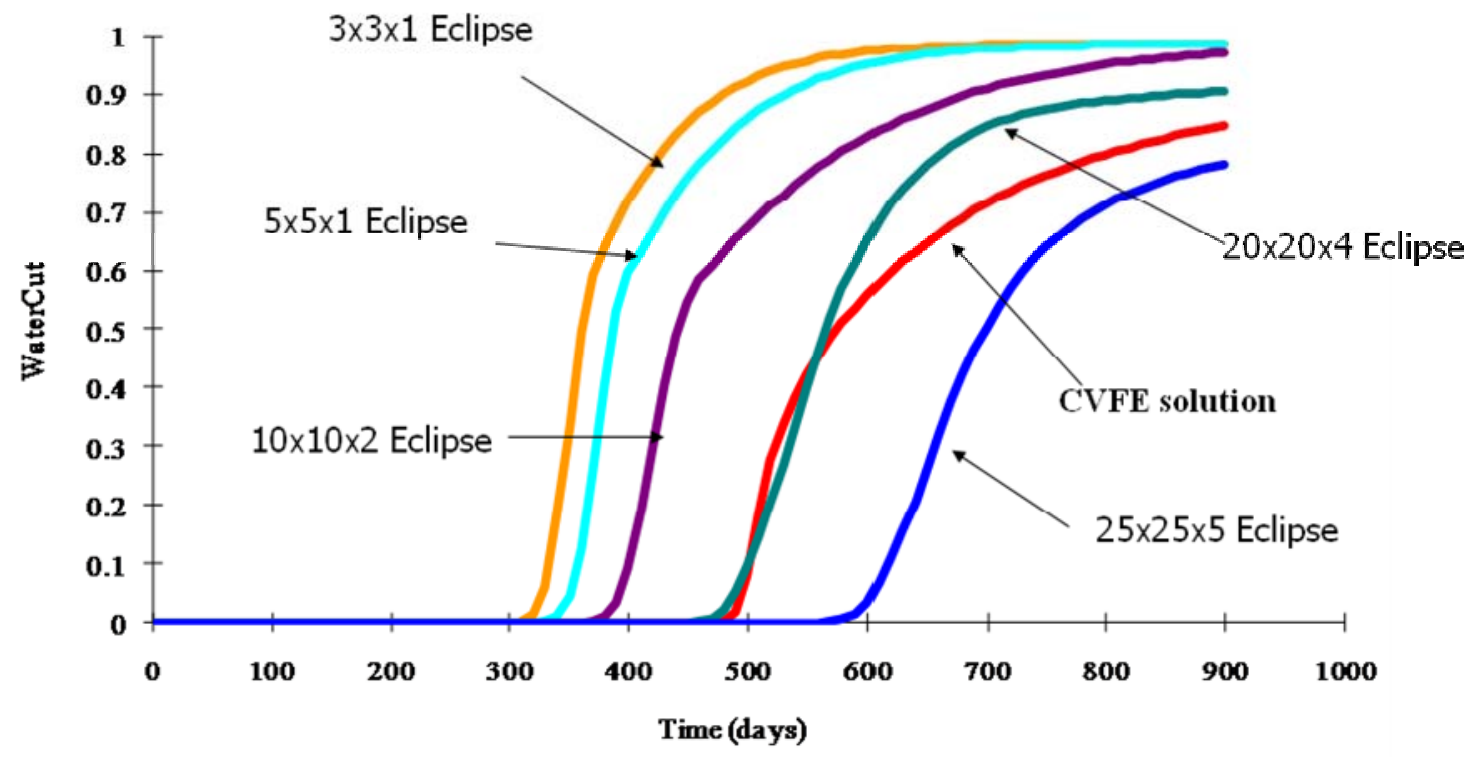

Figure 4.15 Water cut comparisons among homogenized ECLIPSE simulations and CVFE simulation

Figure 4.16 qualitatively demonstrates the limitation of Oda's method in fine grid blocks. If a fracture only penetrates a small section of a grid block, the grid block permeability tensor calculated from Oda's method is very small. Under this small permeability, fluid cannot flow through that grid block. This explains the reason for nonconvergence phenomena observed in 
finer grid block cells. Therefore, during this type of homogenization process, it becomes essential to choose the right grid-block sizes. CVFE simulator is one option for helping to choose grid block sizes. Another method is discussed in the next section on the conventional FD simulator approach.

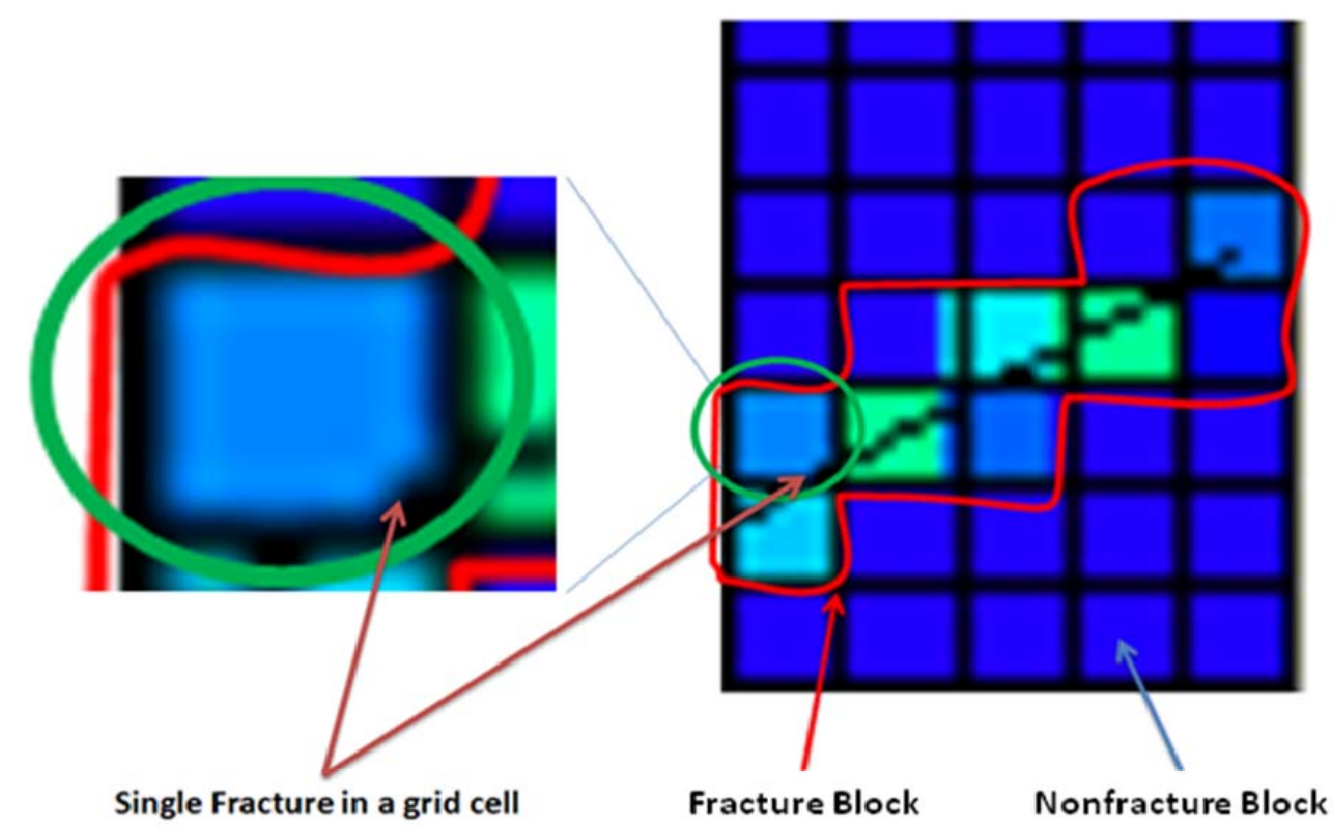

Figure 4.16 Limitation of Oda's permeability tensor calculation with fine grid cells

\section{Fine Grid for Conventional Finite-Difference Simulator of ECLIPSE}

Fracture density/intensity analysis is a popular method for studying fractured reservoir flow behaviors by conventional reservoir simulators such as ECLIPSE. The case study proposed here is to make very fine grid blocks $(100 x 100 x 20,10-\mathrm{ft}$ cube, as shown in Figure 4.17) to separate fracture-occupied grid and nonfracture-occupied grid blocks for the "real" basement system. Then based on the intensity of fracture weighting in the fracture-occupied grid, the porosity of that grid will be calculated for a single porosity model. Assuming that the transmissivity (kh value) of that grid block were the same as the DFN characterized fracture value. This fine-grid 
characterized, single porosity basement model helps elucidate the maximum accuracy of conventional fractured reservoir flow simulation achieved by ECLIPSE. Results from this study were compared with the CVFE simulation results.

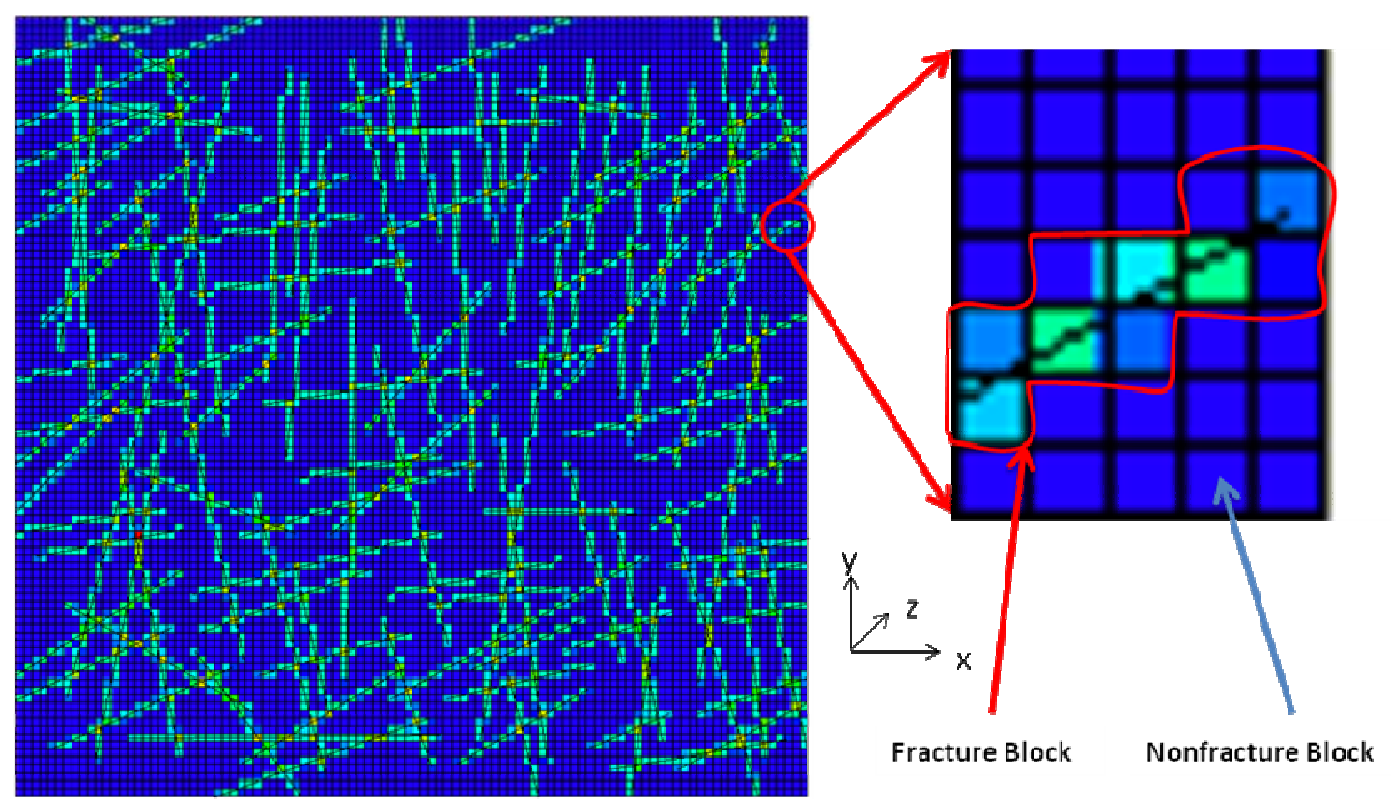

Figure 4.17 Illustration of simplified permeability constrain calculations

$$
\Phi_{\text {GridBlock }}=\frac{V_{D F N}}{V_{\text {GridBlock }}}=\frac{\sum\left(A_{D F N} \cdot e\right)}{V_{\text {GridBlock }}}=P_{32} \cdot e
$$

Figures 4.18 to Figure 4.20 show the comparisons between results from the case of fine grid (100x100x20) porosity homogenization and CVFE. Figure 4.20 shows that each single fracture/fault in the basement reservoir model is clearly represented by the single grid block row/column in this finer grid ECLIPSE model. The oil saturation distribution fronts/residuals are basically identical with the CVFE results (from Figure 4.18 and 4.19). Furthermore, the oil production rate and water cut are basically matched with the solution from CVFE results in a reasonable range. The difference is possibly caused by uncorrected permeability tensor and the 
flow path in the fracture/fault grid. For example, the single fracture/fault is not parallel to the grid block row/column in the conventional finite-difference approach.

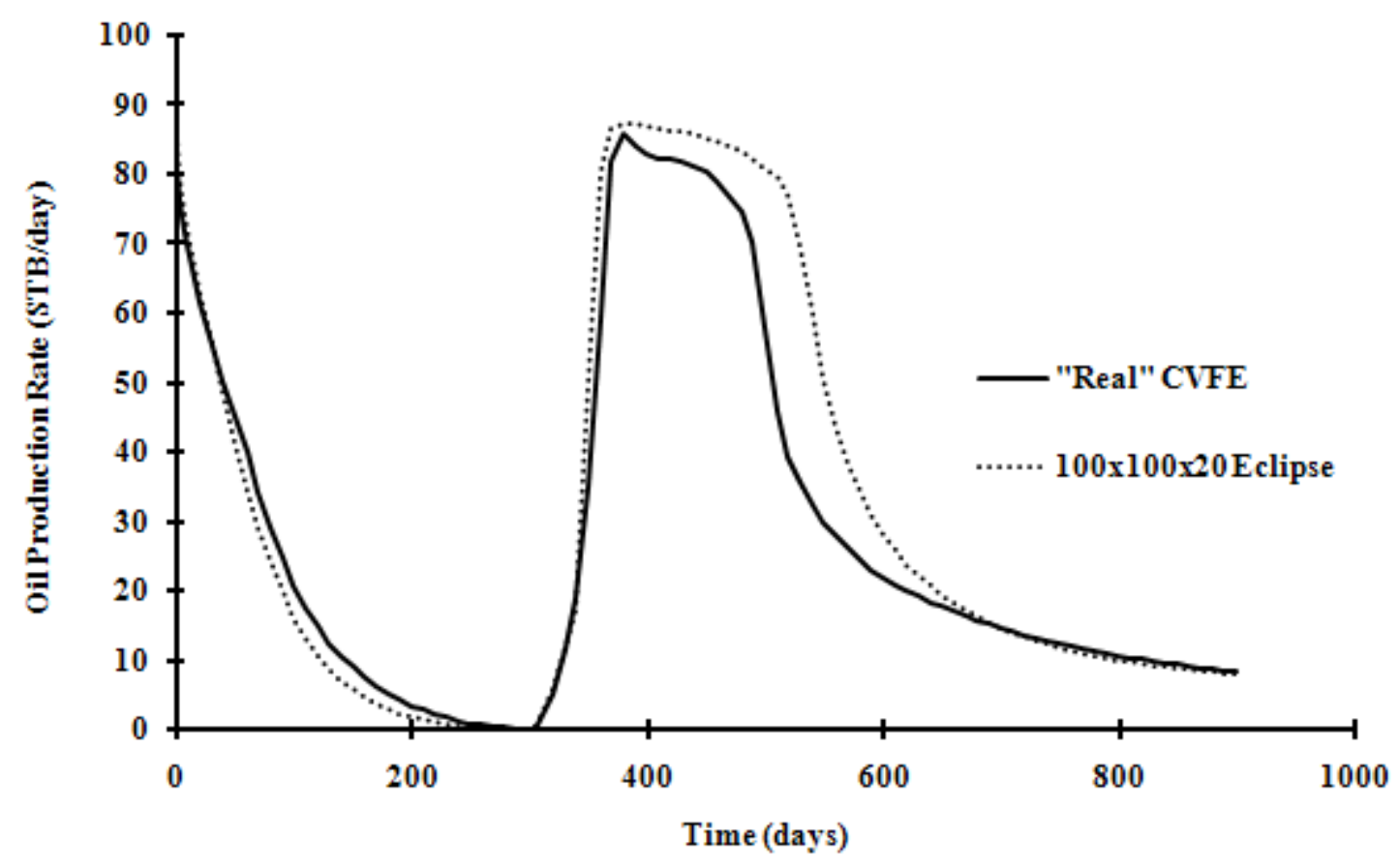

Figure 4.18 Oil production rate comparisons among homogenized (simplified) ECLIPSE simulation and CVFE simulation

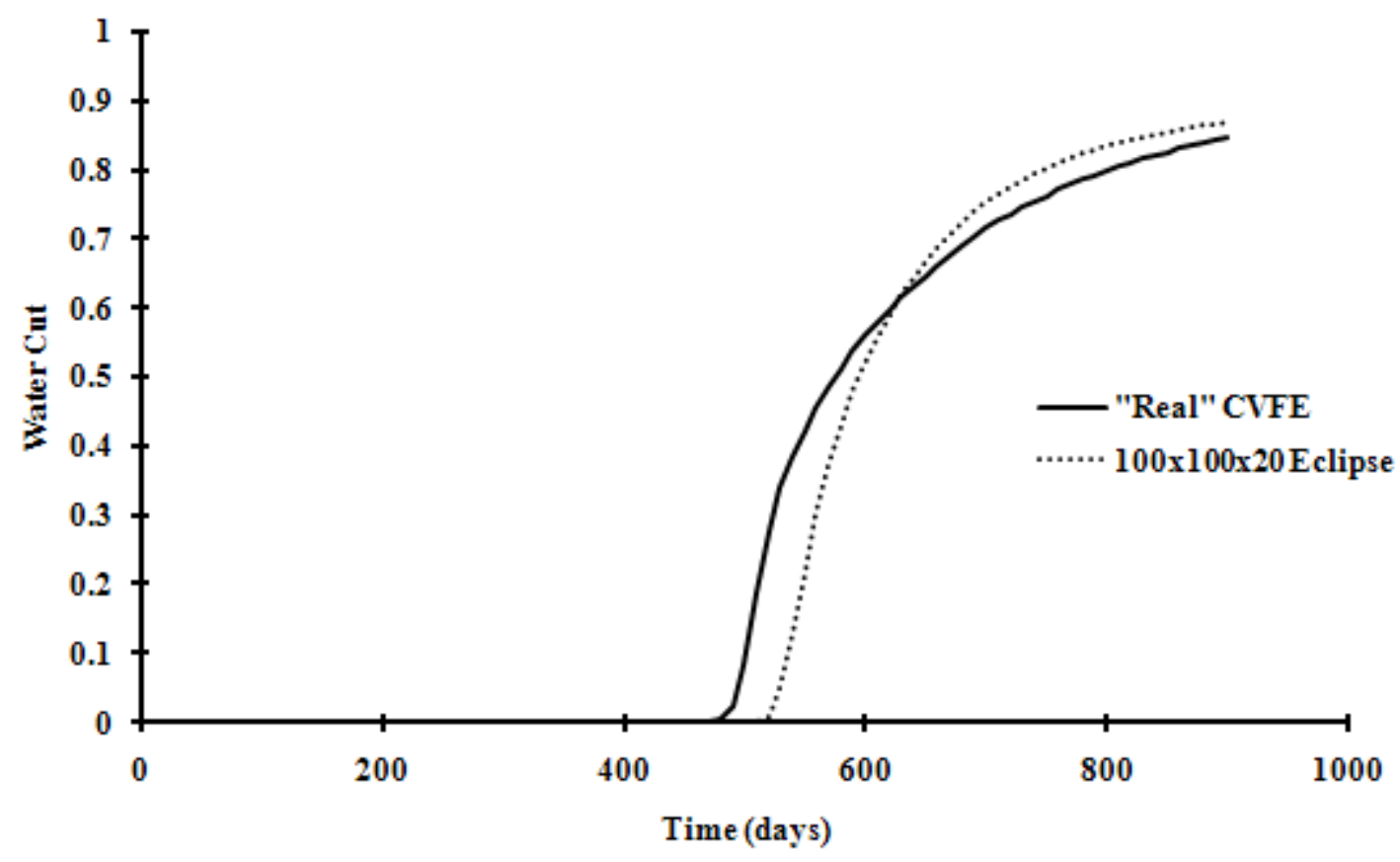


Figure 4.19 Water cut comparisons among homogenized (porosity only) ECLIPSE simulations and CVFE simulation

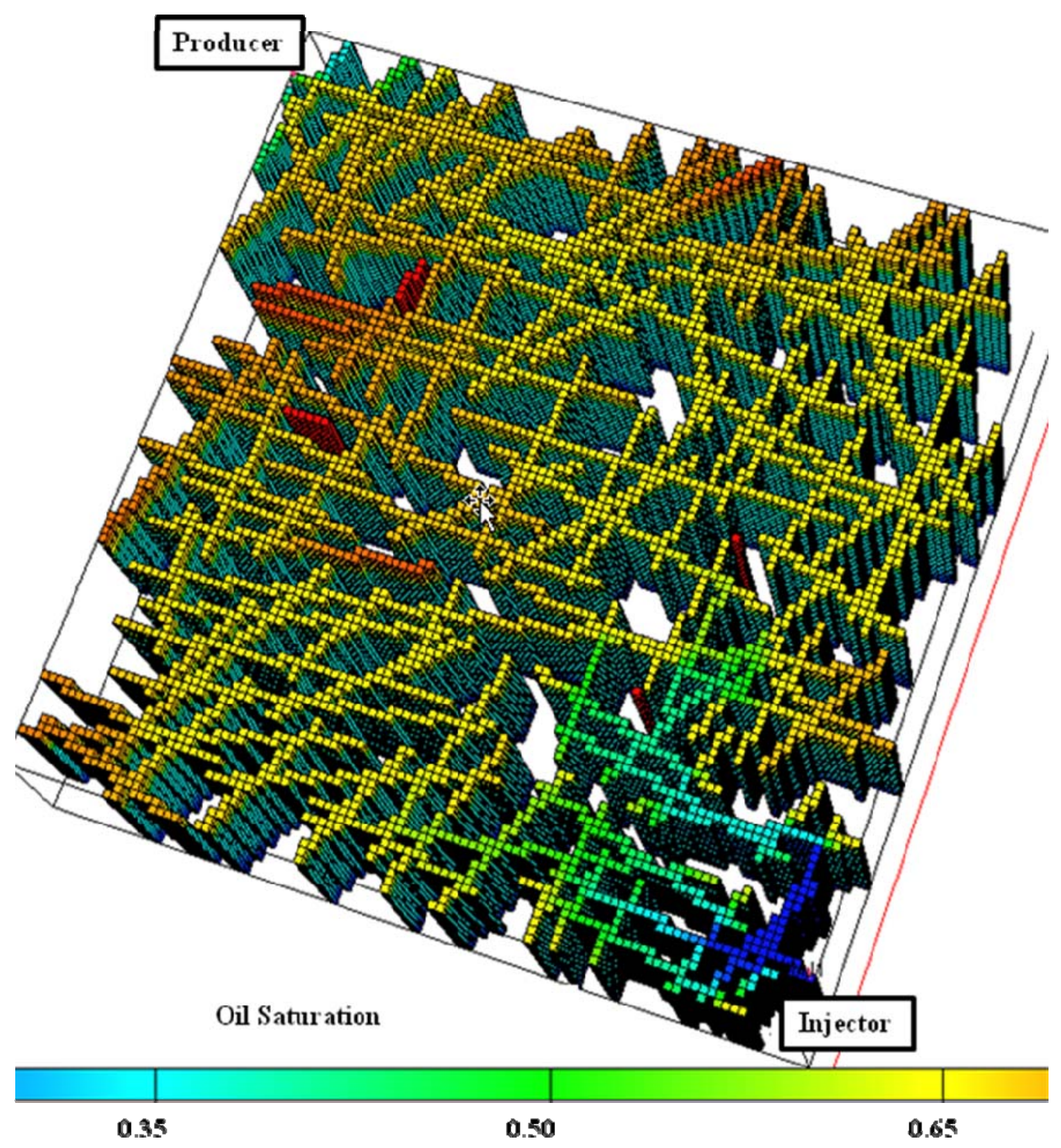

Figure 4.20 Oil saturation distributions for simplified fracture homogenization simulation (at day 900) by ECLIPSE 
Figure 4.21 shows that the flow path through the homogenized grid block is longer than the DFN modeled fluid flow path. Compared to the CVFE results, this longer flow path partly explained the later water breakthrough by homogenized grid simulation.

Since the only reservoir driving force was coming from the decreased pressure at the primary production stage, the oil production rate was almost identical for both the CVFE and the ECLIPSE simulations. During secondary production, water flooding becomes the major reservoir driving force. Figure 4.18 shows that both simulations reached a similar highest production rate, but the rate from the homogenized simulation is a little higher than the rate from the CVFE simulation. In this study, the homogenized grid-block permeabilities are just simply implied by constraining constant $k h$ value from the fracture permeability. Mathematically, this simplified permeability is somewhat higher than its true value. This explains why the oil production rate in homogenized finite-difference simulation is higher than the rate from CVFE simulation.

Study in this section helped in understanding the basement reservoir in the following ways:

1) Running the fine-grid, porosity-homogenized, DFN-modeled basement reservoir through a conventional finite-difference simulator achieved reasonable accuracy as compared to the CVFE direct-DFN simulation results.

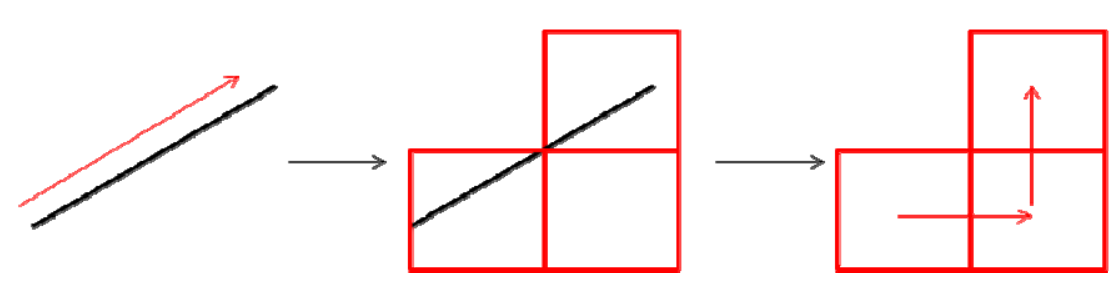

Figure 4.21 Fluid flow through fracture/fault represented by the equivalent grid blocks (DFN $\rightarrow$ homogenized DFN $\rightarrow$ homogenized grid blocks)

2) From the aspect of simulation efficiency, the CVFE simulator revealed its advantage over the homogenized ECLIPSE simulator: in the case of three-phase black-oil 
basement reservoir with 900 days simulation time, the CVFE simulator requires 28 minutes cluster time rather than 3259 minutes required by the ECLIPSE simulator.

\section{Chapter Summary}

This chapter was focused on upscaling the DFN-characterized fracture network model to its equivalent model. While the basement fracture network was made more irregular, results from DFN simulations differed considerably from the regularized ECLIPSE equivalent simulations. The ECLIPSE simulator cannot precisely simulate highly irregular basement fracture networks. However, the irregular fracture networks can be homogenized using a variety of approaches for the ECLIPSE simulator. These approaches include the geometric-based Oda method and the flow-based "Block K" method. The Oda method was applied to all of the homogenization studies in chapter. If the results from DFN simulation are correct, the results from the Oda homogenization show great sensitivity to grid-block size. Surprisingly, convergence was not achieved with finer grid-block size. This is due to the Oda method of permeability upscaling, and homogenization is more accurate when the single fracture penetrates the entire block diagonally (maximum penetration). A simple geometric homogenization scheme involved with fine mesh is achieved by assigning the permeability of the fracture to the block that it penetrated. This approach led to the homogenized results matching the DFN simulation results reasonably well but with high computing cost.

\section{Chapter Nomenclature}

$\begin{array}{lll}A_{D F N} & = & \text { fracture area, } \mathrm{ft}^{2} \\ b & = & \text { exponential term in the power law relations } \\ e & = & \text { fracture/fault storage thickness, } \mathrm{ft}\end{array}$




\begin{tabular}{|c|c|c|}
\hline$e_{i}$ & $=$ & $\mathrm{i}^{\text {th }}$ fracture storage thickness inside of certain grid cell, $\mathrm{ft}$ \\
\hline$h_{x}, h_{y}, h_{z}$ & $=$ & grid block thickness, $\mathrm{ft}$ \\
\hline $\mathbf{K}$ & $=$ & permeability tensor, md \\
\hline$k_{x x}, k_{y y}, k_{z z}$ & $=$ & principal permeability, md \\
\hline$l_{i}$ & $=$ & $i^{\text {th }}$ fracture length inside of certain grid cell, $\mathrm{ft}$ \\
\hline$p$ & $=$ & fluid pressure, psi \\
\hline $\mathrm{P}_{32}$ & $=$ & fracture intensity as fracture area per unit volume, $\mathrm{ft}^{2} / \mathrm{ft}^{3}$ \\
\hline $\mathrm{P}_{33}$ & $=$ & fracture intensity, fracture volume per unit volume, $\mathrm{ft}^{3} / \mathrm{ft}^{3}$ \\
\hline $\mathbf{q}$ & $=$ & flux vector \\
\hline$V_{D F N}$ & $=$ & fracture/fault volume, $\mathrm{ft}^{3}$ \\
\hline$V_{\text {GridBlock }}$ & $=$ & grid block volume, $\mathrm{ft}^{3}$ \\
\hline$x \_\min$ & $=$ & minimum $x$ value in the power law relations, $\mathrm{ft}$ \\
\hline$\Phi$ & $=$ & porosity \\
\hline$\Phi_{\text {GridBlock }}$ & $=$ & grid block porosity \\
\hline$\sigma$ & $=$ & shape factor in the dual porosity model \\
\hline$\rho$ & $=$ & density, $\mathrm{lbm} / \mathrm{ft}^{3}$ \\
\hline$\mu$ & $=$ & viscosity, cp \\
\hline$\delta_{i j}$ & $=$ & Kronecker coefficient \\
\hline
\end{tabular}




\section{Chapter Bibliography}

Aavatsmark, I., Barkve, T. and Mannseth, T.: "Control Volume Discretization Methods for 3D Quadrilateral Grids in Inhomogeneous, Anisotropic Reservoirs”, paper SPE 38000, (1997)

Dershowitz, B., LaPointe, P., Eiben, T., Wei, L., "Integration of Discrete Feature Network Methods with Conventional Simulator Approaches”, paper SPE 49069 (1998)

FRED User's Mannual, Golder Associate (2007)

Fu, Yao, "Multiphase Control Volume Finite Element Simulations of Fractured Reservoirs," Ph.D Dissertation, University of Utah, Salt Lake City (2007)

Fu, Y., Yang, Y.-K., Deo, M., “Three-Dimensional, Three-Phase Discrete-Fracture Reservoir Simulator Based on Control Volume Finite Element (CVFE) Formulation,” paper SPE 93292, (2005)

Fung, L.S., Hiebert, A.D., and Nghiem, L.X., "Reservoir Simulation with a Control Volume Finite Element Method", paper SPE 21224 (1991)

Horne, R., and Raghavan, R., “Reservoir Description and Dynamics”, JPT (Match 2004) 32-33

Lee, J., Kasap, E., and Kelkar, M.G., “Analytical Upscaling of Permeability for 3D Gridblocks”, paper SPE 27875 (1994)

Liu, Y., Harding, A., Abriel, W., Strebelle, S., "Multiple-point Simulation Integrating Wells, Three-Dimensional Seismic Data and Geology", AAPG Bulletin (July 2004) v.88, No. 7, 905921

Matthäi, S.K., Mezentsev, A., Belayneh, M., "Control-Volume Finite-Element Two-Phase Flow Experiments with Fractured Rock Represented by Unstructured 3D Hybrid Meshes”, paper SPE 93341 (2005)

Ronald A. Nelson, "Geologic Analysis of Naturally Fractured Reservoirs", Gulf Professional Publishing, second edition (2001)

Sukirman, Y.B. and Lewis, R.W.: "Three-Dimensional Fully Coupled Flow: Consolidation Modeling Using Finite Element Method”, paper SPE 28755, (1994)

Verma, S. and Aziz, K.: “A Control Volume Scheme for Flexible Grids in Reservoir Simulation”, paper SPE 37999, (1997)

Yang, Yi-kun, "Finite-element Multiphase Flow Simulation," Ph.D Dissertation, University of Utah, Salt Lake City, UT (2003)

Young, L.C.: “An Efficient Finite Element Method for Reservoir Simulation”, paper SPE 7413, (1978) 


\section{Chapter 5. Multiscale Representation of Fracture Networks and Simulation}

\section{Table of Contents}

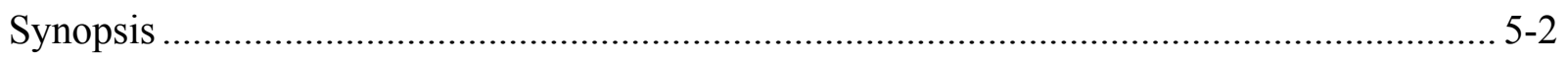

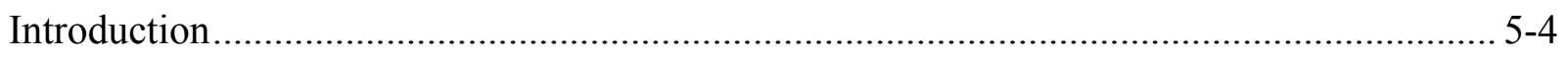

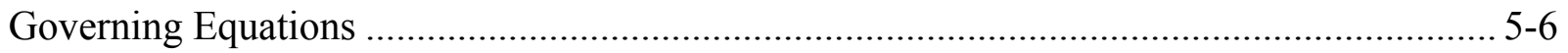

Modeling the Fractured Basement Reservoir …………................................................ 5-6

The Seismic and Subseismic Basement Reservoir Geological Model ............................... 5-6

Other Model Parameters ................................................................................................. 5-8

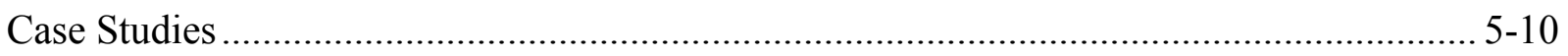

Base Case Simulations to Choose the Production Well Operating Strategy ….................. 5-10

OOIP Distribution Based on Thicknesses of Seismic and Subseismic Features ............... 5-14

Depth Dependent Reservoir Porosity and Permeability Distributions ............................. 5-19

Depth Dependent Reservoir Properties with Proportional OOIP Distributed in Seismic and

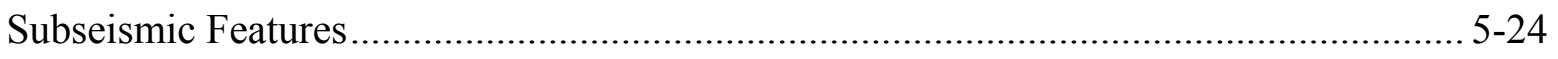

Production Rate Effects with Depth Dependent Reservoir Properties and Proportional OOIP

Distributed in Seismic and Subseismic Features ............................................................. 5-29

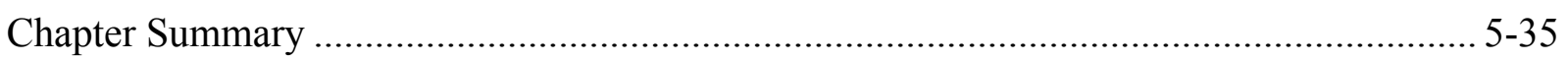

Chapter Nomenclature ………………………………......................................... 5-38

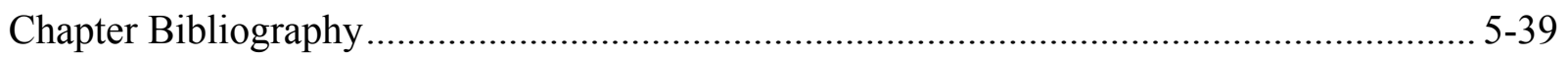




\section{Synopsis}

Due to the presence of highly heterogeneous, complex fracture networks, low permeability/porosity matrix with high permeability fractures, naturally fractured basement reservoirs have been known within the oil and gas industry for many years but were generally regarded as having "no economical potential." These reservoirs have now become legitimate targets due to an ability to better characterize the fracture networks, and understand the mechanics of flow. Since almost all the oil resides in fracture networks, discrete fracture network (DFN) model is one of the best ways to characterize the basement type reservoir. However, there are still some open questions about how to simulate the DFN modeled basement reservoir. Basement reservoirs are often modeled using maps of faults imaged using the seismic method. Only large features are imaged and the reservoir is constructed using these trace maps. In discrete fracture network modeling, we are representing the significant fractures and faults as discrete features rather than as equivalent continua. Among the basic questions that we are addressing are:

1) How does fracture geometry and particularly fracture heterogeneity affect water oil displacement in the reservoir, and

2) Does rate affect recovery?

Given the influence of heterogeneity on water-oil displacement we are also looking at the influence of different heterogeneity types. In particular we are interested in identifying the influences of fractures that are not detected by seismic exploration. We refer to the fractures and fractures zones that are detected by seismic exploration as "seismic" fractures. However, we know that there are more fractures within the reservoir that may contribute to both storage and connections. These undetected, but potentially significant fractures are the "subseismic" fractures. 
In this study, a DFN based conceptual seismic and subseismic basement reservoir model was constructed with two vertical parallel seismic scale features and 117 uniform area subseismic scale features with different orientations. The seismic features were 1489 feet X 1489 feet. The area of each subseismic feature is about $3.86 \%$ of the seismic feature. All seismic and subseismic features are well connected. The distance between the two seismic features is $1000 \mathrm{ft}$. This conceptual model could be treated as a portion of a giant basement reservoir model with granite/basalt formations. Five sets of simulations have been performed in order to study water oil displacement mechanisms for such a reservoir to address the following central questions.

1) What influence does the subseismic feature permeability have on displacement? In this set, simulations with different subseismic feature permeabilities were compared.

2) How does oil distribution in seismic/subseismic features affect recovery and water cut behavior? This is one of the most pertinent questions in the recovery of oil from basement reservoirs. In these sets of simulations, the distribution of oil in the seismic and subseismic features was varied.

3) How does the system behave when porosities and permeabilities are depth dependent? Often, in basement systems, porosities and subsequently permeabilities are lower at increasing depths. What are the characteristics of recovery under these realistic conditions?

4) How does rate influence recovery? This is another important question that relates to the operational aspects and reservoir management.

Constant seismic feature bottom water support is applied in all simulations. 


\section{Introduction}

Seismic measurements are used to map the locations of the major features in basement reservoirs. These features can be brought into a DFN model for the multiphase flow simulations purpose. Will et al. (2005) reported a method of integration of seismic anisotropy attributes with reservoir-performance data for characterization of naturally fractured reservoir using DFN approach. However, it is recognized that the seismic mapping technique is able to pick out features larger than a certain size. Since most of the basement reservoirs exist in the deep formations (over 2000 meters), most high frequency seismic waves will be lost during the seismic reflecting survey and cannot reach the objective basement formation. In most cases, low frequency seismic method is adopted as the method to do basement reservoir seismic survey. The low frequency seismic waves usually result in limited detection and resolution scale and during interpretation only large size faults in the basement reservoirs are observed. Due to this, the smaller subseismic scale features cannot be represented in conventional deterministic modeling (Araujo et al., 2004). But subseismic scale features do exist in the basement reservoirs. One of the primary questions is: what is the relative importance of the subseismic features in oil storage and displacement?

Other than the seismic and subseismic issues, characterization of the petrophysical properties of fractured basement reservoirs is challenging. Behrenbruch et al. (1995) report large variations in oil properties of basement reservoirs offshore of Vietnam with depth varied from 1750 to 2440 meters. Sibbit (1995) quantified porosity from well logs in fractured basement reservoir and estimated permeability from that field. Li et al. (2004) describes that permeability is one of the most difficult parameters to assess in a reservoir and it is even more challenging when it is the basement reservoir. They also report on a method to quantitatively evaluate basement reservoir's 
permeability based on image logs with the integration of other open-hole logs, mud gas data, drilling data, dynamic well testing and production logging data.

In the DFN (Dershowitz et al., 1998; Basquet et al, 2005; Araujo et al., 2004) approach, the geometry of the fracture network is explicitly modeled and a realistic way of modeling basement reservoir performance is provided. However, these DFN descriptions are generally too detailed and geometrically complex to be simulated by conventional reservoir simulators. Some research has been done by connecting DFN with a dual-porosity model for the case of single-phase flow by Dershowitz (1998). This approach is limited to single-phase flow and no multiphase flow study has been reported.

One serious consideration for the production of oil from basement reservoirs is excess water production which not only reduces the artificial lift efficiency, but also damages the oil zones (Chan et al., 2006). A detailed study of multiphase flow in basement reservoirs has not been reported.

In this study, an upstream flux weighted three-dimensional, two-phase (oil, water) black oil CVFE simulator is developed and employed (Yang, 2003; Fu et al., 2005). This CVFE formulation has been validated and verified through various methods: indexing method (Chapter 3); manufactured solution method (Yang, 2003; Fu et al., 2005); core-scale verifications with Pooladi-Davish and Firoozabadi's (2000) fracture water level experiment (Chapter 6). The fluxbased upstream-weighting function on CVFE discretization formulation is good at handling rotatable permeability tensor. This makes the direct accurate simulation of DFN modeled fractured basement reservoir possible. 
With the same fluid-rock data set and boundary conditions, five sets of multiphase simulations were performed on the same basement reservoir domain based on various assumptions related to the seismic and subseismic features' characteristics.

\section{Governing Equations}

The equations describing compressible two-phase flow are obtained by combining Darcy's law and mass conservation for each phase, and have been discussed earlier. The only difference between three-phase and two-phase systems is the existence of the gas phase. Since the dynamics of water injection was the subject of this study, two-phase, oil-water system was used.

\section{Modeling the Fractured Basement Reservoir}

\section{The Seismic and Subseismic Basement Reservoir Geological Model}

As Figure 5.1 shows, the conceptual seismic and subseismic scale basement reservoir model is composed of two vertical parallel seismic scale features and two sets of well-connected subseismic scale features. There are two seismic features and two sets of subseismic scale features. Detailed feature information is summarized in Table 5.1.

This domain was constructed under the FRED working environment. The isolated fractures were removed during the domain construction. HyperMesh was applied for the domain meshing. Adaptive meshing method was chosen to have the complex area fine meshed but have the simple area with the coarse meshed. 


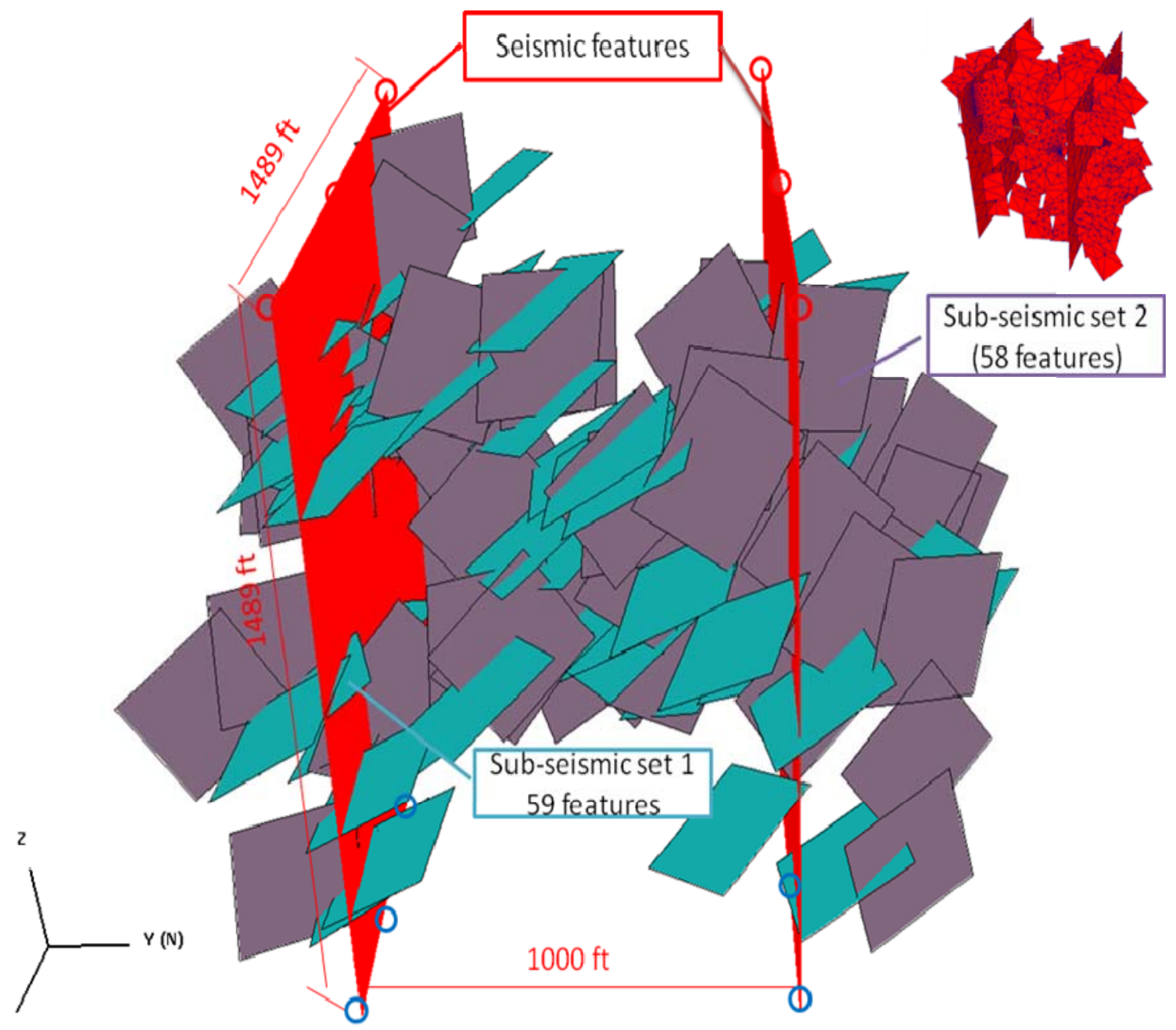

Figure 5.1 Illustration of the basement reservoir domain composed by seismic features and subseismic features

Table 5.1 Summary of seismic and subseismic features

\begin{tabular}{|c|c|c|c|}
\hline & Seismic features & Subseismic set 1 & Subseismic set 2 \\
\hline Number of features & 2 & 59 & 58 \\
\hline Total feature area $\left(\mathrm{ft}^{2}\right)$ & 4433290 & 5046262 & 4960732 \\
\hline Mean orientation & 180,0 & 0,60 & 240,0 \\
\hline $\begin{array}{l}\text { Equivalent Radius mean } \\
\text { (ft) }\end{array}$ & 840 & 165 & 165 \\
\hline Equivalent radius std dev & $1.5 \mathrm{E}-5$ & $5.3 \mathrm{E}-6$ & $3.5 \mathrm{E}-6$ \\
\hline Area mean $\left(\mathbf{f t}^{2}\right)$ & 2216645 & 85530 & 85230 \\
\hline
\end{tabular}

Based on the above feature summary, the area of each subseismic feature is about $3.86 \%$ of each seismic feature. Seismic feature area occupied $30.7 \%$ of total area and the rest $69.3 \%$ belongs to 
subseismic features. The feature thicknesses are varied from case to case for the purpose of adjusting reservoir storage. It will be addressed in the separate case studies later. If the same thickness is used for all the features, the storage in the reservoir will be distributed in proportion to the area (which is about 30/70 from seismic to subseismic size features).

\section{Other Model Parameters}

Other than the geological model, there are several other factors that influence the behavior of a producing reservoir, which must be incorporated in the modeling effort to understand their relative importance. Several property sets are required in order to perform the multiphase flow simulations.

1) Reservoir property model: the key to DFN simulation is the assignment of reservoir properties (porosity, permeability, thickness, compressibility) to the features of the geologic model. These properties are either homogeneous or they are varied according to geologic expectations as discussed below.

2) Phase model: this includes two-phase (water and oil) properties such as end-points on the relative permeability curves and initial fluid saturations.

3) Fluid property model: fluid properties include fluid viscosity, compressibility, density, and formation volume factor. 
Table 5.2 Summary of reservoir data for modeling seismic and subseismic featured basement reservoir

\begin{tabular}{|c|c|c|}
\hline Simulator & CVFE & \\
\hline $\begin{array}{l}\text { Grid information } \\
\text { Number if nodes } \\
\text { Number of triangular elements }\end{array}$ & $\begin{array}{l}3690 \\
6846 \\
\end{array}$ & \\
\hline $\begin{array}{l}\text { Fluid property } \\
\gamma\left(\mathrm{lbf} / \mathrm{ft}^{3}\right)\end{array}$ & $\begin{array}{l}\text { Water } \\
62 \\
\end{array}$ & $\begin{array}{l}\text { oil } \\
53 \\
\end{array}$ \\
\hline Fluid data & Water properties ${ }^{*}$ & Oil properties ${ }^{*}$ \\
\hline Reference pressure ( $\mathrm{p}_{\text {ref, }}$ psi) & 14.7 & 4850 \\
\hline $\begin{array}{l}\text { formation volume factor } \mathrm{B}_{\mathrm{r}} @ \\
\text { reference pressure }(\mathrm{rb} / \mathrm{stb}) \\
\text { Compressibility } \mathrm{C}_{\mathrm{B}}(1 / \mathrm{psi})\end{array}$ & $\begin{array}{l}1.0 \\
3 \text { e- } 6\end{array}$ & $\begin{array}{l}1.17 \\
1 \mathrm{e}-6\end{array}$ \\
\hline $\begin{array}{l}\text { Water viscosity }\left(\mu_{\mathrm{r}}\right) @ \text { reference } \\
\text { pressure }(\mathrm{cp}) \\
\text { c }(1 / \mathrm{psi})\end{array}$ & 0.3 & 1.0 \\
\hline
\end{tabular}

\begin{tabular}{|c|c|c|c|}
\hline \multicolumn{4}{|l|}{ Rock-fluid data } \\
\hline \multicolumn{4}{|l|}{ Oil-water system } \\
\hline $\mathrm{S}_{\mathrm{w}}$ & $\mathrm{k}_{\mathrm{rw}}$ & $\mathrm{K}_{\mathrm{ro}}$ & $\mathrm{p}_{\text {cow }}(\mathrm{psi})$ \\
\hline 0.20 & 0.00 & 1.00 & 7.0 \\
\hline 0.30 & 0.07 & 0.40 & 4.0 \\
\hline 0.40 & 0.15 & 0.125 & 3.0 \\
\hline 0.50 & 0.24 & 0.0649 & 2.5 \\
\hline 0.60 & 0.33 & 0.0048 & 2.0 \\
\hline 0.80 & 0.65 & 0.00 & 1.0 \\
\hline 0.90 & 0.81 & 0.00 & 0.2 \\
\hline \multicolumn{4}{|l|}{ Initial conditions } \\
\hline $\mathrm{P}(\mathrm{psi})$ & \multicolumn{3}{|l|}{4300} \\
\hline $\mathrm{S}_{\mathrm{w}}$ & \multicolumn{3}{|l|}{0.2} \\
\hline Boundary & \multirow{3}{*}{\multicolumn{3}{|c|}{$\begin{array}{l}\text { Constant seismic feature bottom water pressure support: } \\
6 \text { water injection wells on the bottom of two seismic features ( } 3 \text { on } \\
\text { each) which are operated as } 4500 \text { psi bottom-hole pressure (BHP) } \\
\text { control. } \\
\text { Nonflow boundary conditions other than seismic feature bottoms }\end{array}$}} \\
\hline conditions & & & \\
\hline & & & \\
\hline Production wells & \multicolumn{3}{|c|}{$\begin{array}{l}6 \text { production wells are chosen on the top of seismic scale features ( } 3 \text { on } \\
\text { each seismic feature, two on the corners and one on the middle, as } \\
\text { shown on the geological model illustrations (red circled on the top)) }\end{array}$} \\
\hline
\end{tabular}

* Reservoir fluid properties (pressure related) are calculated by the following equations:

$$
\begin{array}{r}
B=B_{r} \exp \left[-C_{B}\left(P-P_{r e f}\right)\right] \\
\mu=\mu_{r} \exp \left[-C_{\mu}\left(P-P_{r e f}\right)\right]
\end{array}
$$


4) Boundary conditions: the behavior of the model may be strongly influenced by the boundary conditions that are assumed for the fractured basement. The boundaries are usually considered as nonflow or constant pressure features.

5) Discretized mesh: in the numerical simulation the DFN based reservoir domain has to be meshed into triangles along each feature. The conformal nodes and sides of intercrossed features have to be honored for the purpose of fluid flow computations.

Some reservoir properties are varied in different case studies and the details will be discussed later. Other reservoir parameters which were held constant throughout the simulations are summarized in Table 5.2.

As shown in Table 5.2, the bottom supporting water boundary conditions could either be treated as water injectors or the bottom aquifer support. Some type of water aquifer support is evidenced in a number of basement reservoirs world-wide.

\section{Case Studies}

\section{Base Case Simulations to Choose the Production Well Operating Strategy}

In this model, we assume both seismic and subseismic features have constant porosity (30\%) and thickness. A set of simulations were carried out to pin down the operating conditions. The initial average reservoir pressure was set at 4300 psi with 4500 psi bottom water support on the seismic features. The vertical drop was about $1500 \mathrm{ft}$ equivalent to a hydraulic head of about 675 psia. A 4500 psi bottom water support pressure will give about 3825 psi static pressure at the top of the reservoir. Under these conditions, the bottom-hole pressures at which the producers operate will have to be determined. In these simulations, the reservoir has about 0.72 MMSTB (million stock tank barrels) oil in place. Three different production pressures, 3500/2500/1500 psi, were 
examined with two sets of fracture permeabilities. In set 1 seismic and subseismic feature have the same permeability $-100,000 \mathrm{md}$. and in set 2 the seismic features have 100,000 md permeability and the subseismic features have 1,000 md permeability). Therefore, there are six simulations in this base case study in total, which are identified in Table 5.3.

The following questions are being addressed in these simulations.

1) Do operating parameters significantly impact the production behavior?

2) Even when the properties are homogeneous, does a permeability contrast (between seismic and subseismic features) make a significant difference in performance?

The oil recovery comparisons, shown in Figure 5.2, depict two distinct sets of oil recovery results due to the difference in permeability of subseismic features. Obviously, higher subseismic feature permeability results in higher oil recovery at a given time.

Table 5.3 Summary of reservoir properties and operating conditions for case 1 set of simulations

\begin{tabular}{|c|c|c|c|c|c|c|}
\hline \multicolumn{2}{|c|}{ K1A } & \multirow{2}{*}{$\frac{\mathrm{K1B}}{0.3}$} & \multirow{2}{*}{$\frac{\mathrm{K1C}}{0.3}$} & \multirow{2}{*}{$\frac{\text { K3A }}{0.3}$} & \multirow{2}{*}{$\begin{array}{l}\text { K3B } \\
0.3\end{array}$} & \multirow{2}{*}{$\begin{array}{l}\text { K3C } \\
0.3\end{array}$} \\
\hline \multirow{4}{*}{$\begin{array}{l}\text { Porosity } \\
\text { Thickness (ft) } \\
\text { Permeaiblity (md) } \\
\text { (seismic) } \\
\text { (subseismic) }\end{array}$} & 0.3 & & & & & \\
\hline & 1.4 & 1.4 & 1.4 & 1.4 & 1.4 & 1.4 \\
\hline & 100,000 & 100,000 & 100,000 & 100,000 & 100,000 & $\begin{array}{l}100,00 \\
0\end{array}$ \\
\hline & 100,000 & 100,000 & 100,000 & 1,000 & 1,000 & 1,000 \\
\hline $\begin{array}{l}\text { Producer's pressure } \\
\text { (psi) }\end{array}$ & 3,500 & 2,500 & 1,500 & 3,500 & 2,500 & 1,500 \\
\hline
\end{tabular}




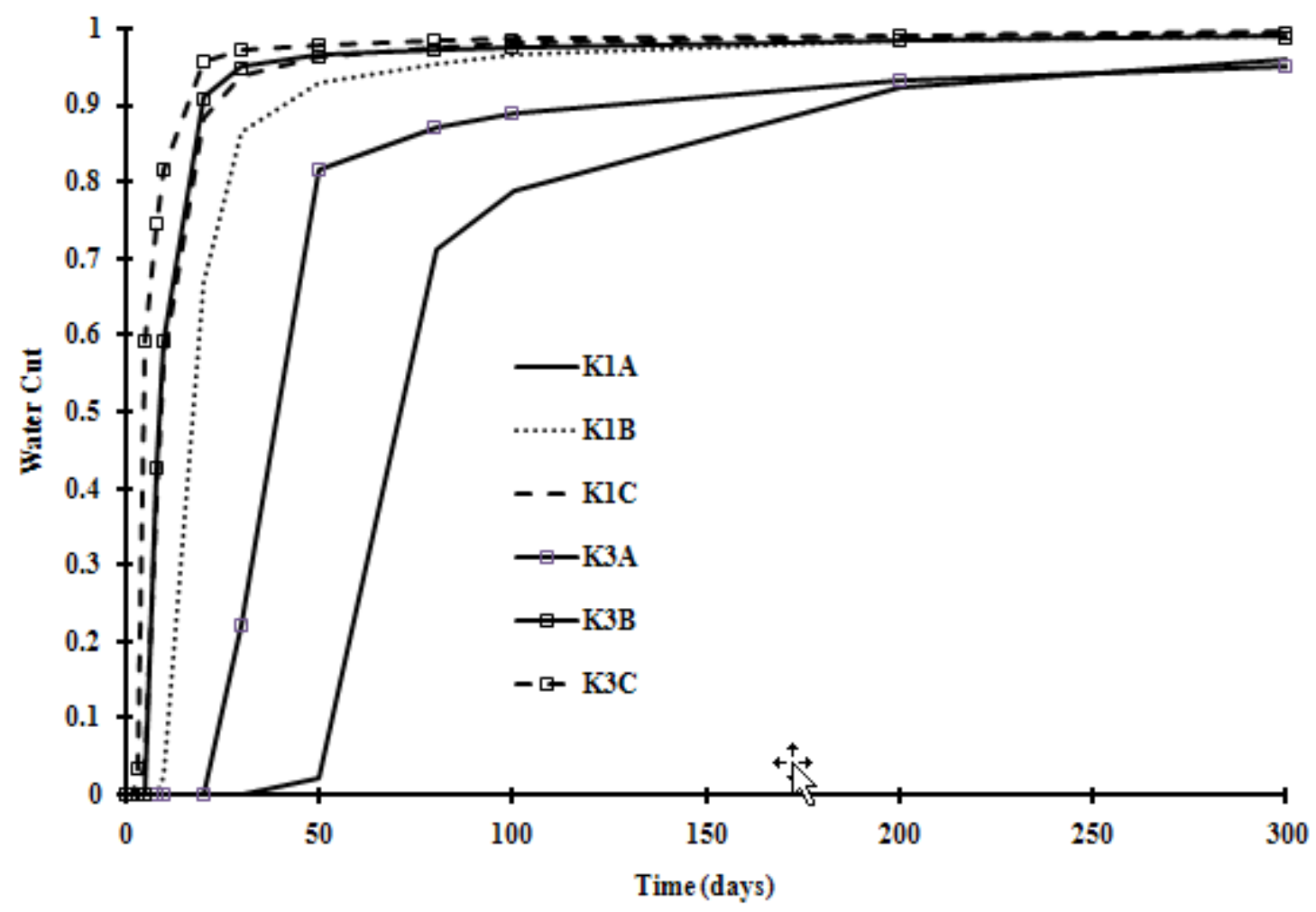

Figure 5.2 Comparison of oil recovery for case 1 set of simulations

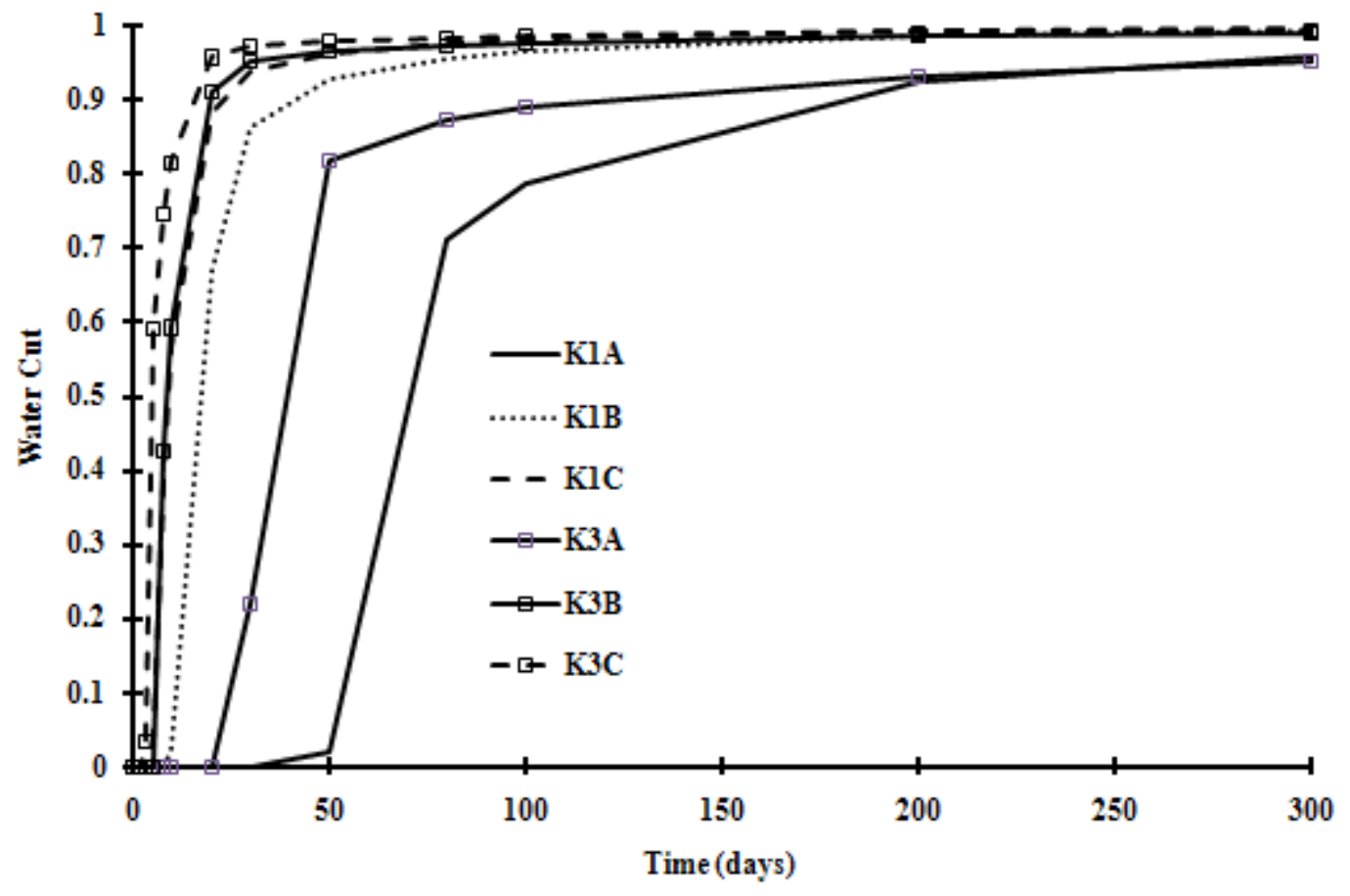

Figure 5.3 Comparison of water cut versus for case 1 set of simulations 
The water cut picture is a bit more complex (Figure 5.3). The slowest breakthrough is obtained when the subseismic permeability and the producer BHP are the highest. The water arrives early in cases where the permeability and producer pressures are lower because a higher water drawdown is created and the water velocity through the system is higher. Since the water cut on day 2,000 is almost 1 in all the cases, the ultimate recoveries for the six simulations are can be summarized as in Table 5.4.

The high and the low permeability scenarios can be treated as extreme cases of permeability variation in the next sets of simulations. The highest producer pressure was

chosen for further simulations to limit the impact of rate of water rise. The rate effect was studied separately by using explicit production rates in the producers.

In this multiscale system where the properties of each of the subsystems are homogeneous, some observations could be summarized as follows.

1) There is significant impact of the permeability of the subseismic features. Lower subseismic permeabilities result in lower recoveries and quicker breakthroughs for the same drawdown.

2) Recovery is not impacted by drawdown (lower producer pressures), but breakthrough is quicker with a steeper drawdown.

In reservoirs with significant vertical extent, the displacement of oil (the lighter fluid), with water (the heavier fluid) is gravity dominated. The relative magnitude of prevailing gravity forces to viscous forces is given by the gravity number $\left(\mathrm{N}_{\mathrm{G}}\right)$ ( Shook et al., 1992; Kulkarni et al., 2006).

Table 5.4 Case 1 simulation summaries at the end of simulations (day 2,000)

\begin{tabular}{lllllll}
\hline & K1A & K1B & K1C & K3A & K3B & K3C \\
\hline Recovery factor, $R$ & 0.60 & 0.61 & 0.61 & 0.46 & 0.47 & 0.48 \\
\hline
\end{tabular}




$$
N_{G}=\frac{\Delta \rho \cdot g \cdot \mathbf{k} / \emptyset}{\mu_{o} \cdot v}
$$

It is known that as the gravity number increases, so does recovery in gravity dominated systems. Another way of looking at this is that sufficient time is made available for gravity to take effect. As the system permeability decreases, so do the gravity number and the recovery decreases. When the drawdown increases, the velocity increases, reducing the gravity number, but not sufficiently to lower recoveries significantly.

\section{OOIP Distribution Based on Thicknesses of Seismic and Subseismic Features}

All the simulations were performed with a constant BHP of 3500 psi in the producers. The sets of simulations in this section were designed to address the central question of how different oil distributions in the two subsystems impacted recovery. Three different distributions of $80 / 20,50 / 50$ and $20 / 80$ in the seismic and subseismic features were created. These proportional distributions were created by adjusting the thickness of seismic features and subseismic features, even with constant thicknesses for each of those systems.

Since the areas of total seismic and total subseismic are known, and with constant porosity $(30 \%)$ the OOIP distribution is directly proportional to the pore volume or product of area and thickness. These relations could be described from Equation (5-3) to Equation (5-6):

$\mathrm{PV}($ pore volume $)=\mathrm{S}($ Area $) \times$ e (thickness $) \times \phi($ porosity $)$

$\frac{\mathrm{PV}_{\text {seismic }}}{\mathrm{PV}_{\text {Subseismic }}}=\frac{\mathrm{S}_{\text {seismic }} \times \mathrm{e}_{\text {seismic }}}{\mathrm{S}_{\text {Subseismic }} \times \mathrm{e}_{\text {Subseismic }}}$

$$
\mathrm{PV}_{\text {total }}=\mathrm{PV}_{\text {seismic }}+\mathrm{PV}_{\text {subseismic }}
$$




$$
\text { OOIP }=\mathrm{PV} \times \mathrm{S}_{\text {oil }}
$$

Based on the above relations and known parameters, six simulations were built in this case study as shown in Table 5.5. More or less ultimate recoveries were reached by day 2000 and these are shown in Table 5.6. The ultimate oil recoveries have large variations from set to set as shown in Figure 5.4 and Table 5.6. The significant results are discussed below.

Table 5.5 Summary of reservoir properties and operating conditions on case 2 studies

\begin{tabular}{llllll} 
8020K1A & 8020K3A & 5050K1A & 5050K3A & 2080K1A & 2080K3A \\
\hline
\end{tabular}

\begin{tabular}{|c|c|c|c|c|c|c|}
\hline Porosity & 0.3 & 0.3 & 0.3 & 0.3 & 0.3 & 0.3 \\
\hline $\begin{array}{l}\text { Thickness (ft) } \\
\text { (seismic } \\
\text { features) } \\
\text { (subseismic } \\
\text { features) }\end{array}$ & $\begin{array}{l}3.6 \\
0.4\end{array}$ & $\begin{array}{l}3.6 \\
0.4\end{array}$ & $\begin{array}{l}2.3 \\
1.0\end{array}$ & $\begin{array}{l}2.3 \\
1.00\end{array}$ & $\begin{array}{l}0.9 \\
1.6\end{array}$ & $\begin{array}{l}0.9 \\
1.6\end{array}$ \\
\hline $\begin{array}{l}\text { Permeaiblity } \\
\text { (md) } \\
\text { (seismic) } \\
\text { (subseismic) }\end{array}$ & $\begin{array}{l}100,000 \\
100,000\end{array}$ & $\begin{array}{l}100,000 \\
1,000\end{array}$ & $\begin{array}{l}100,000 \\
100,000\end{array}$ & $\begin{array}{l}100,000 \\
1,000\end{array}$ & $\begin{array}{l}100,000 \\
100,000\end{array}$ & $\begin{array}{l}100,000 \\
1,000\end{array}$ \\
\hline
\end{tabular}

Table 5.6 Recovery comparisons from case 2 sets of simulations

\begin{tabular}{lllllll}
\hline & $\mathbf{8 0 2 0}$ & $\mathbf{8 0 2 0}$ & $\mathbf{5 0 5 0}$ & $\mathbf{5 0 5 0}$ & $\mathbf{2 0 8 0}$ & $\mathbf{2 0 8 0}$ \\
& K1A & K3A & K1A & K3A & K1A & K3A \\
\hline Recovery, R & 0.70 & 0.68 & 0.64 & 0.55 & 0.57 & 0.41 \\
\hline
\end{tabular}




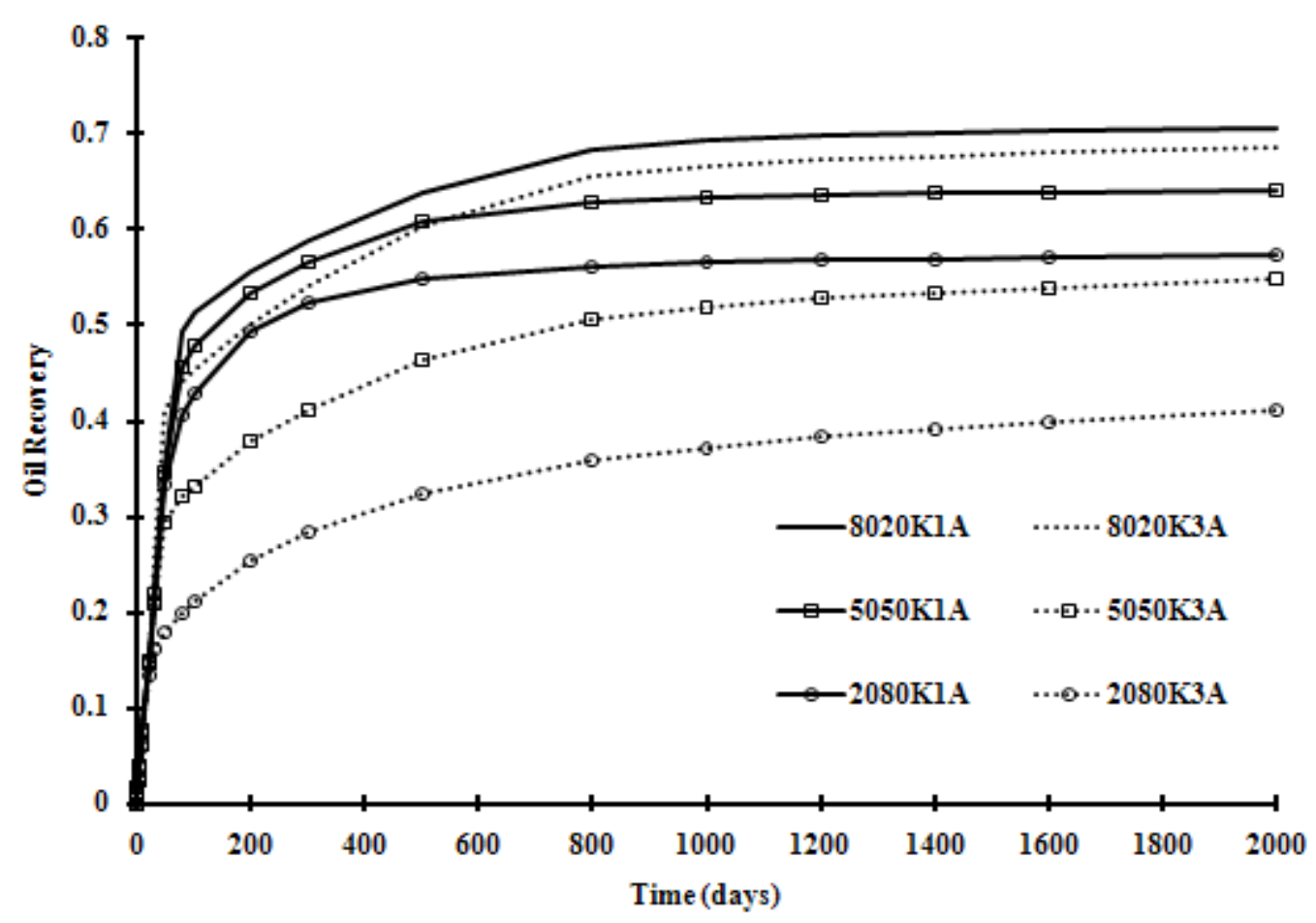

Figure 5.4 Comparisons of oil recovery versus simulation time for case 2 studies.

Comparing different OOIP distributions with same permeability in the seismic and subseismic features:

1) When the permeability is held high $(100,000 \mathrm{md})$, the oil recovery decreases as more oil is distributed into the subseismic features. The gravity number analysis provided earlier was established for conventional matrix-dominated porous media. The gravity number is affected only to the extent to which the Darcy velocity in the system is impacted. As more oil is distributed into the seismic features, the vertical velocity through the system is lower (as indicated by later breakthroughs shown later). However, this decrease is not sufficient to explain the significant decline in recovery (from $71 \%$ at $80 / 20$ to $54 \%$ at $20 / 80$ ). What we are seeing here is the reduction in recovery related to the geometry and connectivity of the fracture system. 
2) When the permeability in the subseismic features is reduced to $1000 \mathrm{md}$, the trend described above becomes even more exaggerated. The recovery decreases from $68 \%$ for the $80 / 20$ distribution to $55 \%$ for the $50 / 50$ distribution, and to $41 \%$ for the $20 / 80$ distribution. Once again this is related the reduced gravity numbers as the velocity through the system increases. But superposed on this is the geometric effect of the fracture network, which becomes more of a factor, as the permeability in the more numerous subseismic features is reduced in comparison to the seismic features.

Comparing different permeability with same OOIP distributions in the seismic and subseismic features:

1) When the subseismic features have only $20 \%$ of the oil, reducing their permeability does not impact recovery significantly, as evidenced by $68 \%$ recovery for the 1000 md case compared to $70 \%$ for the 100,000 case. This should be compared to the previous case study where $70 \%$ of the oil was in the subseismic features and a difference in recovery of about $7 \%$ was observed. The gravity number in these comparisons is affected both by permeability and velocity, and it is surprising to see little change in recovery. This underscores the importance the characterization of fracture networks and their properties.

2) As more and more oil is distributed into the subseismic features the recovery difference between the two permeability cases widens (9\% for 50/50 and $16 \%$ for 20/80). Thus not only knowledge of the hydrodynamic parameters of the system is important (permeability, viscosity, velocity, etc.), but also the fracture characterization and connectivity. 
As shown in Figure 5.5, the water breakthrough times are related directly to the average water advance velocity in the system. As more oil is distributed in the subseismic features or as the permeability of the subseismic feature is reduced, the water advance velocity increases (on the average, since this is a highly complex function of saturation, location and time) leading to earlier breakthroughs. These earlier breakthroughs are the cause of lower recoveries at a given time.

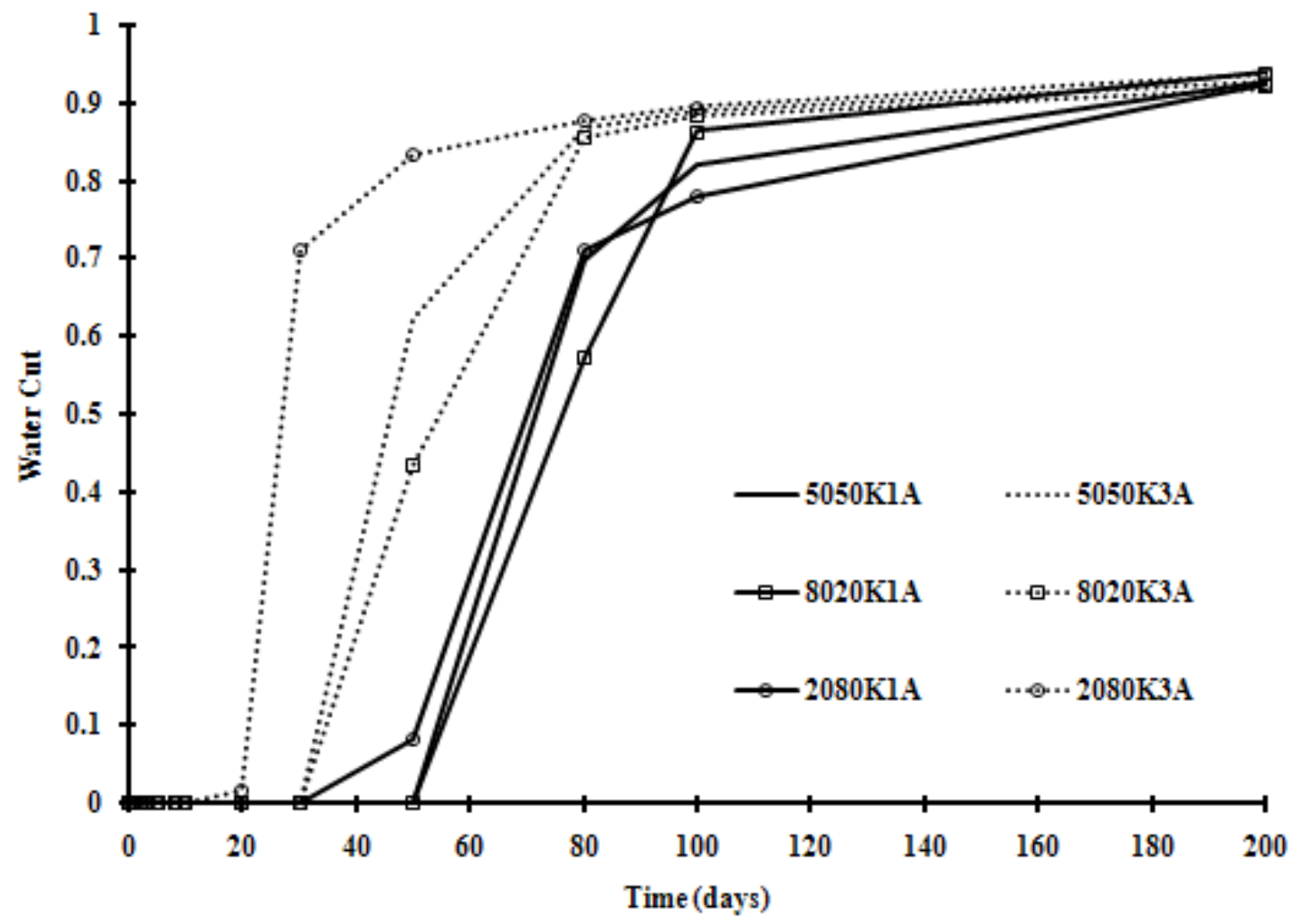

Figure 5.5 Comparisons of water cut versus simulation time for case 2 studies with different time scales. 


\section{Depth Dependent Reservoir Porosity and Permeability Distributions}

Both case studies discussed above have basic assumptions of constant porosity distributions and constant permeability distributions vertically either in seismic scale features or subseismic scale features. Basement reservoirs are highly complex structures, and with the vertical drop of 1,500 $\mathrm{ft}$ they are comprised of different formations and sedimentary layers geologically. The fracture/microfracture porosity changes very strongly with depth as reported by Chan et al. (2006). In this case study, two depth dependent basement porosity models are presented and the permeability is correlated with porosity distributions.

The depth dependent porosity and permeability correlations are shown in the Figure 5.6. For porosity, two models (linear and nonlinear) are presented in the range of $90 \sim 10 \%$ from the top reservoir surface to the bottom of reservoir. Based on the porosity value, permeability of both the seismic and the subseismic features is calculated by correlating with porosity values. An assumption here is the permeability only depends on porosity value no matter the size of the feature. The nonlinear porosity distribution function is defined in Equation (5-7):

$\Phi=0.511405-0.05625 \ln (\mathrm{D})$

where $\emptyset$ is the porosity and D is the depth from the top reservoir surface. This equation ensures that with total depth of $1500 \mathrm{ft}$, the porosity would be distributed from $90 \%$ to $10 \%$ vertically. 


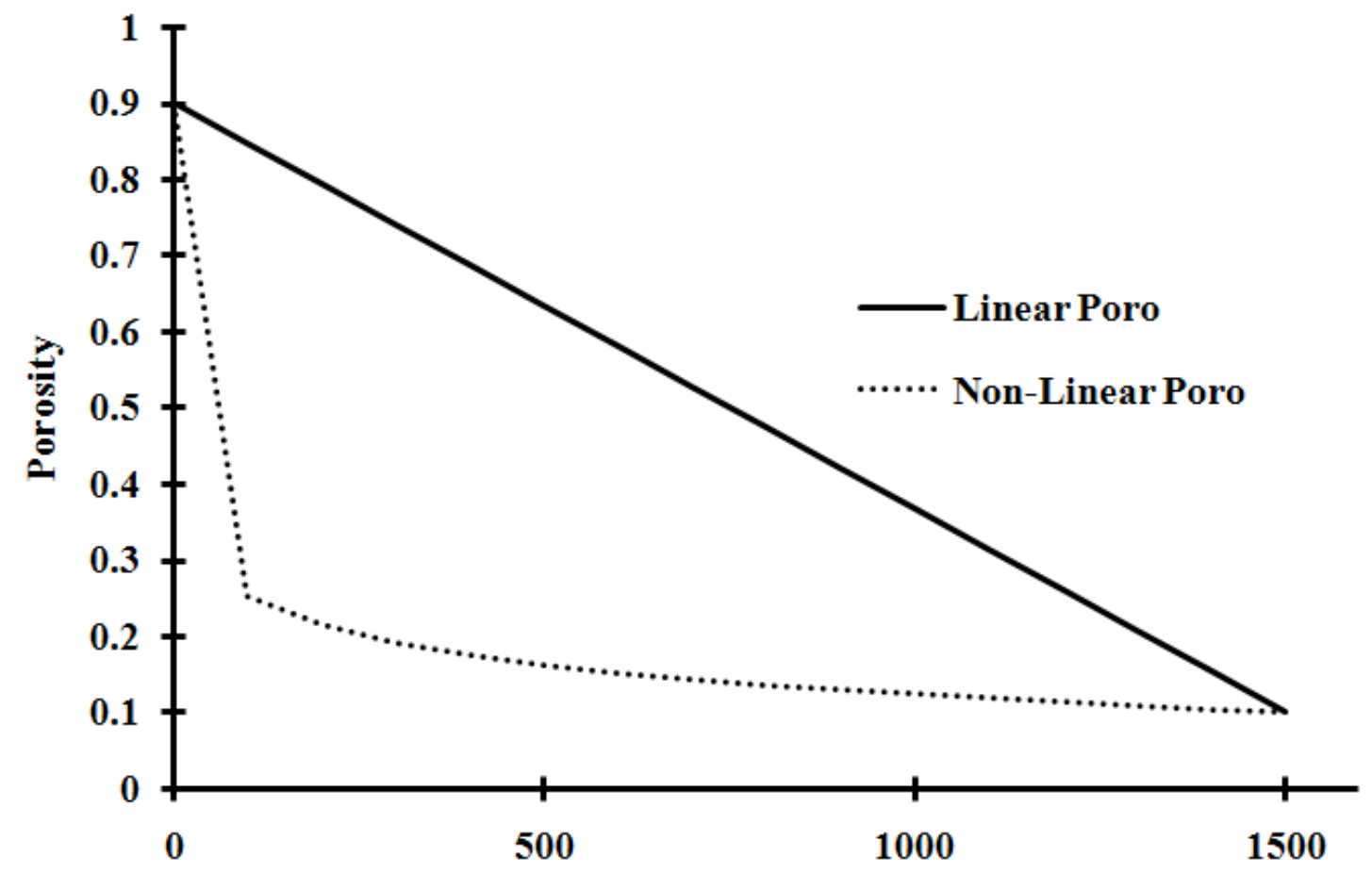

Depth from Top*

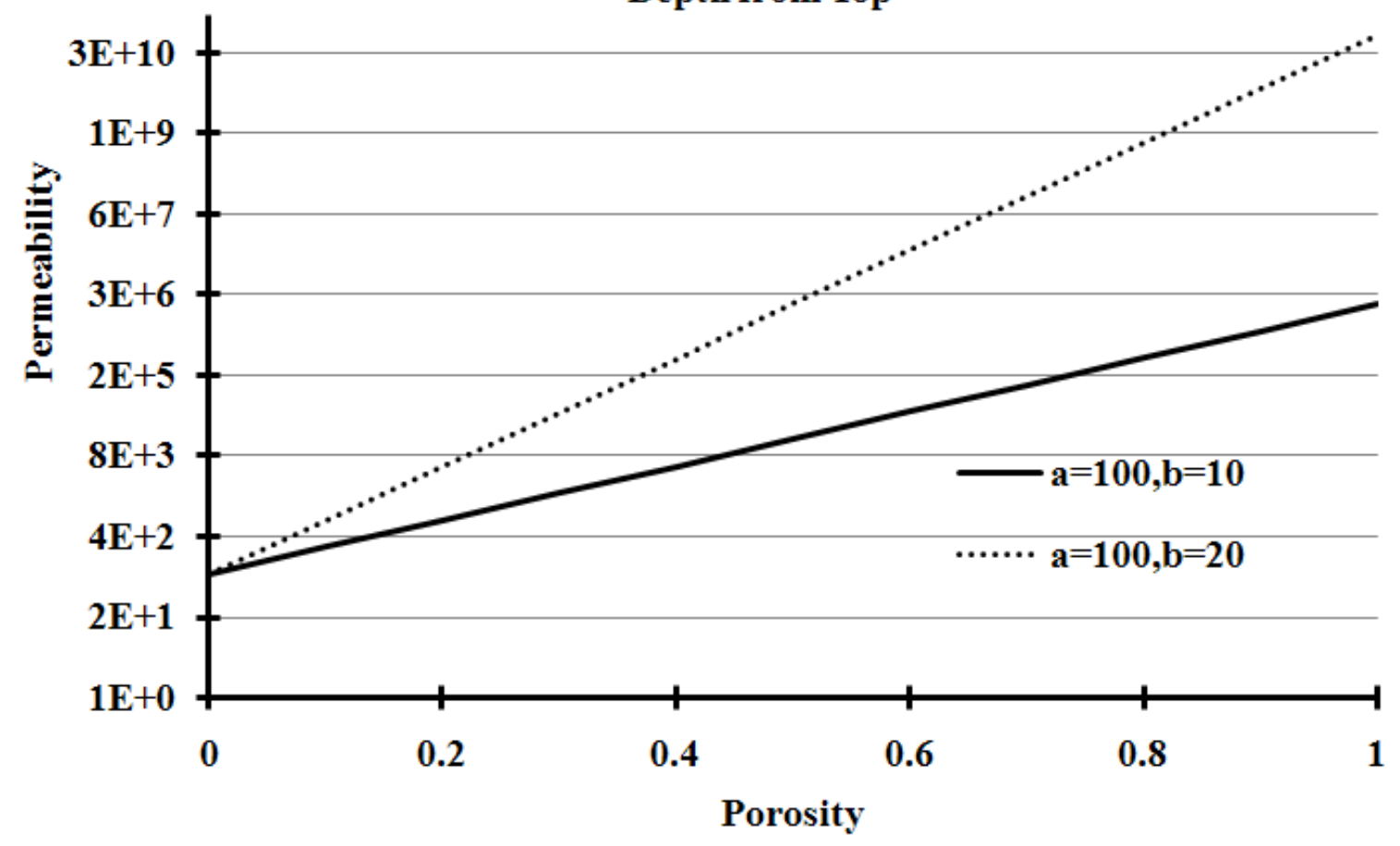

Figure 5.6 Depth dependent porosity and permeability. 
The permeability correlation is defined in Equation (5-8):

$\mathrm{K}=\mathrm{a} * \exp (\mathrm{b} * \Phi)$

where $\mathrm{K}$ is the permeability and $\mathrm{a}$ and $\mathrm{b}$ are the constants as shown in Figure 5.6.

Three different correlations were examined.

1) Linear porosity with permeability correlation $1(a=100, b=10)$;

2) Nonlinear porosity with permeability correlation $1(\mathrm{a}=100, \mathrm{~b}=10)$;

3) Nonlinear porosity with permeability correlation $2(a=100, b=20)$.

The resultant reservoir property distributions in these case studies are summarized in Table 5.7.

The figures of oil recovery and water cut versus time are shown in Figure 5.7 and 5.8. Some reservoir properties have also been shown in the above table. The difference of the upper porosity limit (0.9 (linear porosity) versus 0.46 (nonlinear porosity)) is due to the strong changes of in porosity due to the implementation of nonlinear porosity equation near the top surface. Since the meshed triangle element center coordinates have been used as depth data in those calculations, depth (from top surface) of $2.49 \mathrm{ft}$ from the top surface could result in $46 \%$ porosity with nonlinear porosity distributions and $89 \%$ porosity on the linear case. This is the reason to choose the permeability correlation 2 as another option for nonlinear porosity correlated permeability. This makes simulation "DepthNonLinearK2A" have the same permeability magnitude with "DepthLinearK1A".

Table 5.7 Summary of reservoir properties and operating conditions on case 3 studies:

\begin{tabular}{llll}
\hline & DepthLinearK1A & DepthNonLinearK1A & DepthNonLinearK2A \\
\hline Porosity & $0.1 \sim 0.89$ & $0.1 \sim 0.46$ & $0.1 \sim 0.46$ \\
Permeability (md) & $294 \sim 744759$ & $273 \sim 3520$ & $748 \sim 123884$ \\
Thickness (ft) & 0.8 & 2.79 & 2.79 \\
OOIP (MMSTB) & 0.72 & 0.72 & 0.72 \\
\hline
\end{tabular}




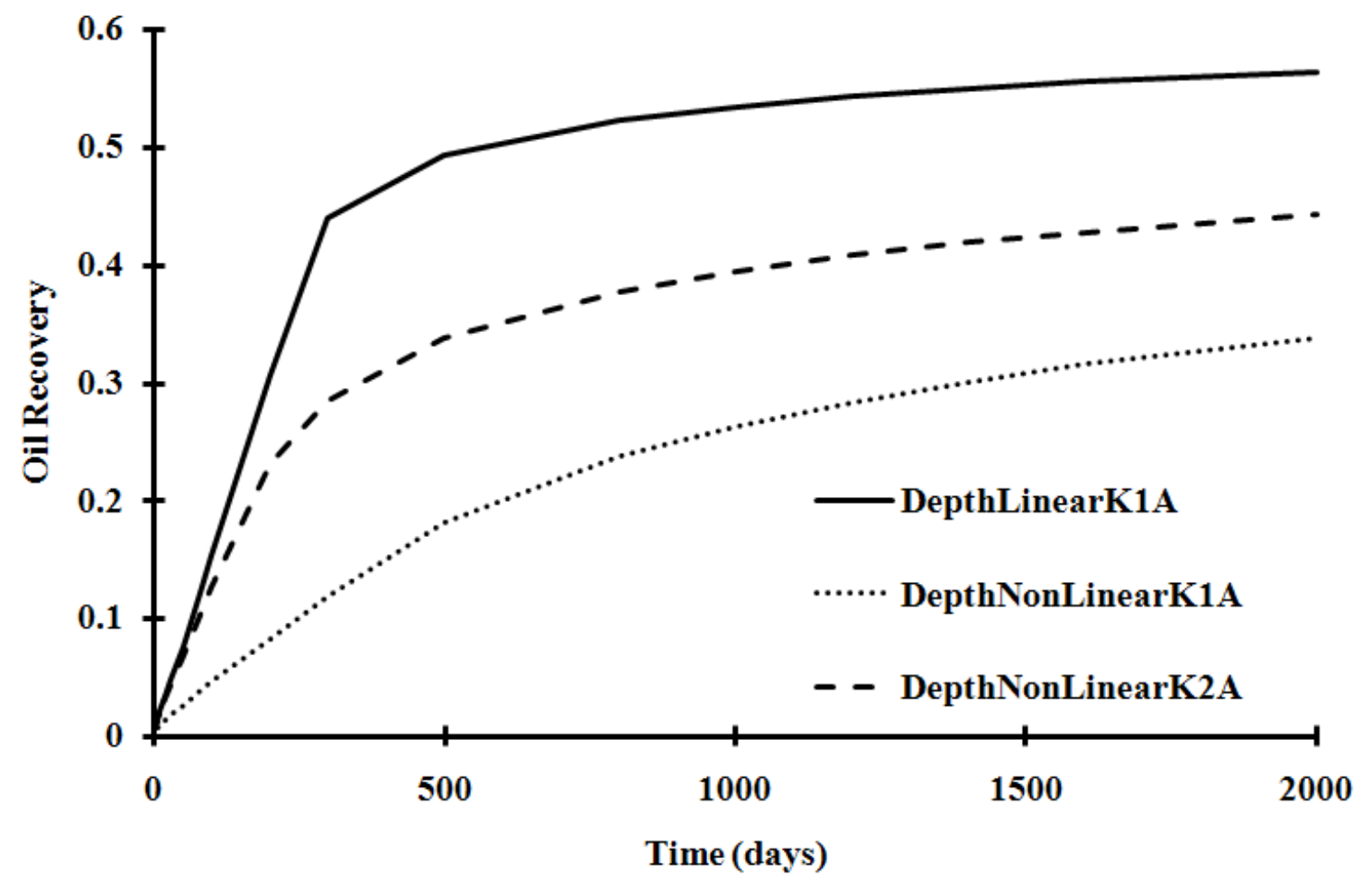

Figure 5.7 Comparisons of oil recovery versus simulation time for case 3 studies.

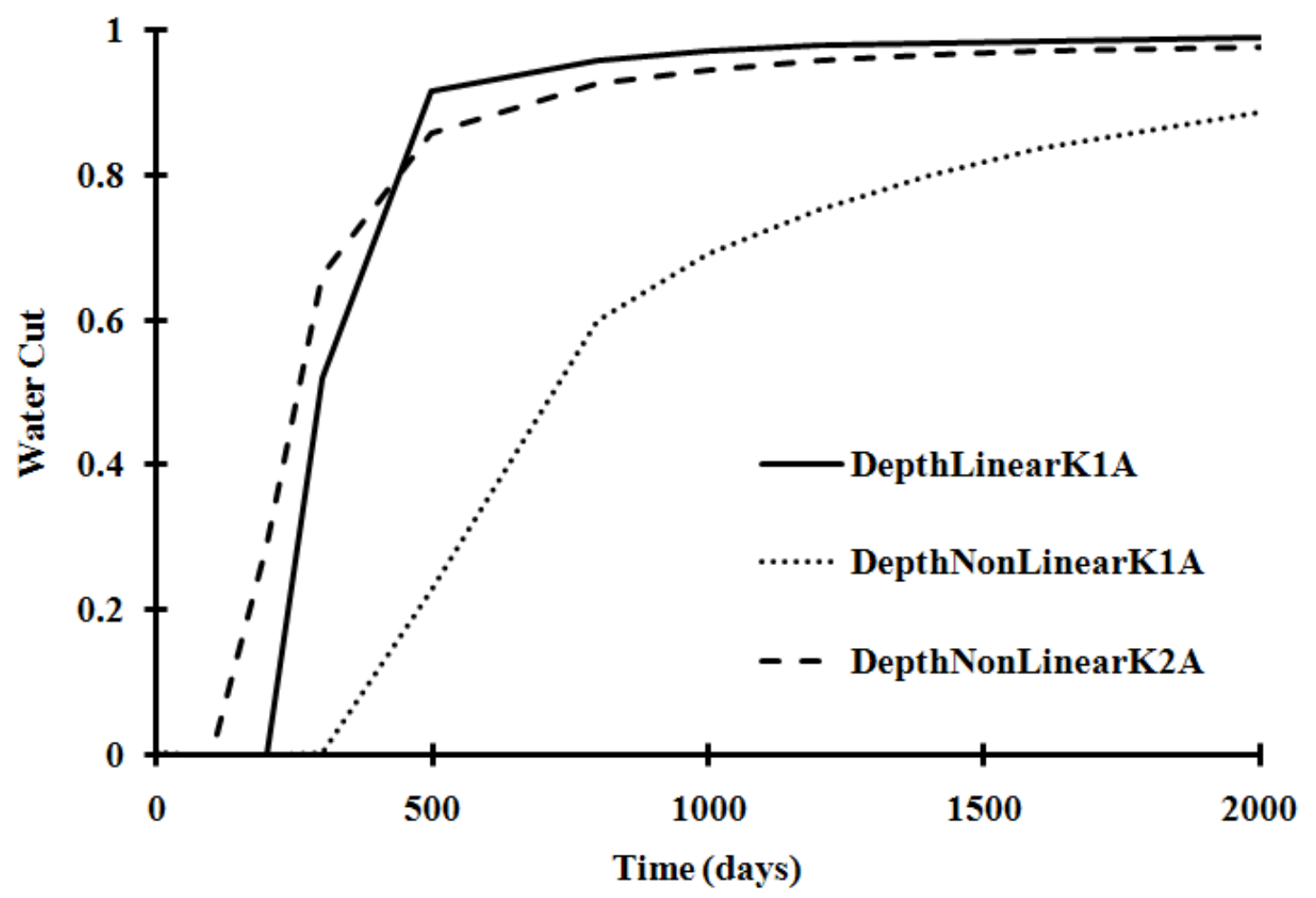

Figure 5.8 Comparisons of water cut versus simulation time for case 3 studies. 
The DepthNonLinearK1A has a very narrow and low permeability distribution. As a result, it has the least recovery, but later breakthrough. It is difficult to characterize the gravity number for these displacements - some sort of permeability averaging will have to be employed. At the end of simulation (day 2,000), the oil recoveries were: DepthLinearK1A (56\% of OOIP); DepthNonLinearK1A (33\% of OOIP); DepthNonLinearK2A (44\% of OOIP).

Figure 5.9 explained the observation of early breakthrough and lower recovery that happened in the case DepthNonLinearK1A. This phenomenon was caused by relative low permeability distributions in the case of DepthNonLinearK1A than in other cases.
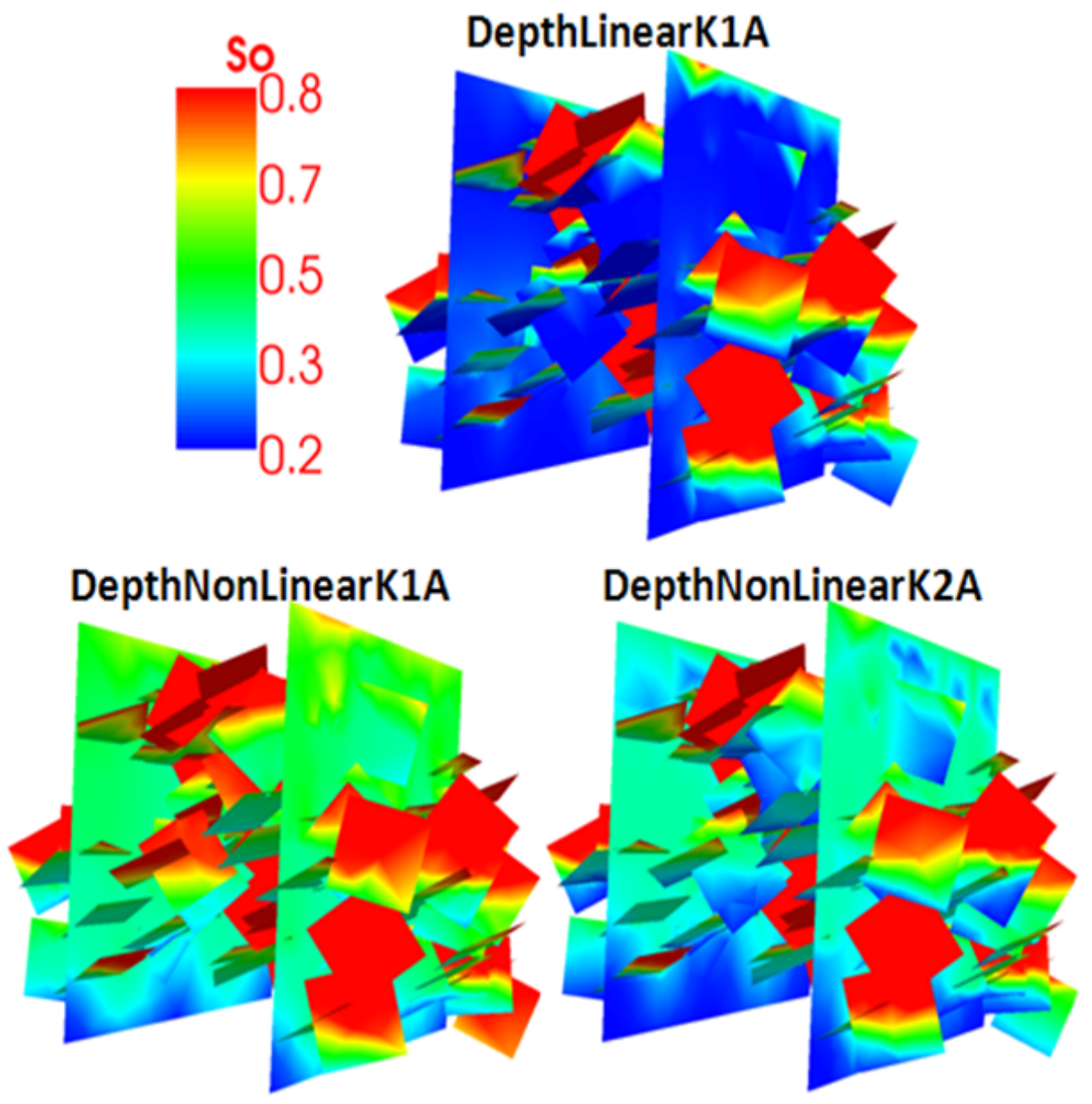

Figure 5.9 Oil saturations at the end of simulation 
Some points could be highlighted from this case study:

1) Realistic depth dependent petro-physical properties can be employed in populating the basement domains created in the DFN models.

2) Increased heterogeneity, particularly with narrow tight distributions peaking at lower permeability values, results in lower recoveries, but later breakthroughs. It is difficult to pinpoint the reason for the later breakthrough with such low recoveries, but it may be related to the narrow, low permeability distribution, and geometric trapping due to this combination.

\section{Depth Dependent Reservoir Properties with Proportional OOIP Distributed in Seismic and Subseismic Features}

Both oil distributions and heterogeneous property distributions had significant impact on oil production from basement systems. In this section, we combine the two effects to see the impact on oil recovery. Distributions are characterized using the relationships from Equation (5-9) to Equation (5-16).

$$
\begin{aligned}
& \text { OOIP }=\mathrm{S}_{\mathrm{oil}} * \mathrm{PV} \\
& \mathrm{S}_{\mathrm{oil}}=20 \%=\text { constant } \\
& \mathrm{PV}=\mathrm{S} * \mathrm{e} * \phi \\
& \mathrm{PV}_{\text {total }}=\mathrm{PV}_{\text {seismic }}+\mathrm{PV}_{\text {subseismic }}
\end{aligned}
$$




$$
\begin{aligned}
& \mathrm{PV}_{\text {seismic }}=\mathrm{S}_{\text {seismic }} * \mathrm{e}_{\text {seismic }} * \emptyset_{\text {seismic }} \\
& =\sum_{\mathrm{i}} \mathrm{S}_{\text {seismic }, \mathrm{i}} * \mathrm{e}_{\text {seismic }, \mathrm{i}} * \emptyset_{\text {seismic }, \mathrm{i}} \\
& \mathrm{PV}_{\text {subseismic }}=\mathrm{S}_{\text {subseismic }} * \mathrm{e}_{\text {subseismic }, \mathrm{i}} * \emptyset_{\text {subseismic }} \\
& =\sum_{\mathrm{i}} \mathrm{S}_{\text {subseismic }, \mathrm{i}} * \mathrm{e}_{\text {subseismic }, \mathrm{i}} * \emptyset_{\text {subseismic }, \mathrm{i}} \\
& \mathrm{e}_{\text {seismic }}=\text { const; } \\
& \mathrm{e}_{\text {subseismic }}=\text { const; }
\end{aligned}
$$

where $\mathrm{S}$ is the area; e is the thickness; $\emptyset$ is the porosity, subscript $\mathrm{i}$ is the discretized finit-element

i. Therefore, the ratio of original oil in place can be defined in Equation (5-17)

$$
\begin{aligned}
& \frac{\text { OOIP }_{\text {seismic }}}{\text { OOIP }_{\text {subseismic }}}=\frac{\mathrm{PV}_{\text {seismic }}}{\mathrm{PV}_{\text {subseismic }}} \\
& =\frac{\sum_{\mathrm{i}} \mathrm{S}_{\text {seismic }, \mathrm{i}} * \mathrm{e}_{\text {subseismic }} * \emptyset_{\text {seismic }, \mathrm{i}}}{\sum_{\mathrm{i}} \mathrm{S}_{\text {subseismi }, \mathrm{i}} * \mathrm{e}_{\text {subseismic }} * \emptyset_{\text {subseismic }, \mathrm{i}}}
\end{aligned}
$$

From Equations (5-9) to (5-17), various OOIP distribution relations can be derived for proportional OOIP distributions between seismic and subseismic features, as shown in Equation (5-18) to Equation (5-20).

$80 / 20(80 \%$ OOIP in seismic features, $20 \%$ in subseismic features):

$$
\frac{\mathrm{e}_{\text {seismic }}}{\mathrm{e}_{\text {subseismic }}}=\frac{4 * \sum_{\mathrm{i}} \mathrm{A}_{\text {subseismic }} \emptyset_{\text {subseismic }}}{\sum_{\mathrm{i}} \mathrm{A}_{\text {seismic }} \emptyset_{\text {seismic }}}
$$


$50 / 50$ (50\% OOIP in seismic feature, $50 \%$ in subseismic features):

$$
\frac{\mathrm{e}_{\text {seismic }}}{\mathrm{e}_{\text {subseismic }}}=\frac{\sum_{\mathrm{i}} \mathrm{A}_{\text {subseismic }} \emptyset_{\text {subseismic }}}{\sum_{\mathrm{i}} \mathrm{A}_{\text {seismic }} \emptyset_{\text {seismic }}}
$$

$20 / 80$ (20\% OOIP in seismic feature, $80 \%$ in subseismic features):

$$
\frac{\mathrm{e}_{\text {seismic }}}{\mathrm{e}_{\text {subseismic }}}=\frac{\sum_{\mathrm{i}} \mathrm{A}_{\text {subseismic }} \emptyset_{\text {subseismic }}}{4 * \sum_{\mathrm{i}} \mathrm{A}_{\text {seismic }} \emptyset_{\text {seismic }}}
$$

The same as in case study 3 , two sets of depth dependent porosity and permeability distributions were chosen in this case study:

1) Linear porosity with permeability correlation $1(\mathrm{a}=100, \mathrm{~b}=10)$ : LPKIA;

2) Nonlinear porosity depth dependent with permeability correlation $1(\mathrm{a}=100, \mathrm{~b}=10)$ : NLPK1A;

3) Nonlinear porosity depth dependent with permeability correlation $1(\mathrm{a}=100, \mathrm{~b}=20)$ : $N L P K 2 A$.

Three property models plus three OOIP distribution relations generate nine simulation sets and are summarized in Table 5.8. Oil recovery and water cut with time are shown in Figures 5.10 and 5.11, and the comparisons are summarized in Table 5.9. Looking at oil recoveries, it appears that for a given property distribution (say NLPK1A), the three OOIP distributions fan out and provide a range of outcomes. At $80 / 20$ in this case, the recovery is highest and breakthrough most delayed, while at 20/80 the recovery the least the breakthrough fastest. If we observe different property distributions at the same OOIP distribution, the tightest distribution with least permeability NLPK1A has the least recovery, but the late section (30/70 distribution). Low recovery coupled with late breakthrough is an unexpected anomaly and is probably related to 
geometric trapping. It could also be due to the fact that under low permeability conditions, water influx is lower, leading to low water advance velocities. The spread between the linear distribution with a broader distribution and high median permeability and the tight nonlinear distribution increases as more oil is distributed in subseismic features.

Table 5.8 Summary of reservoir properties and operating conditions for case 4 sets of simulations

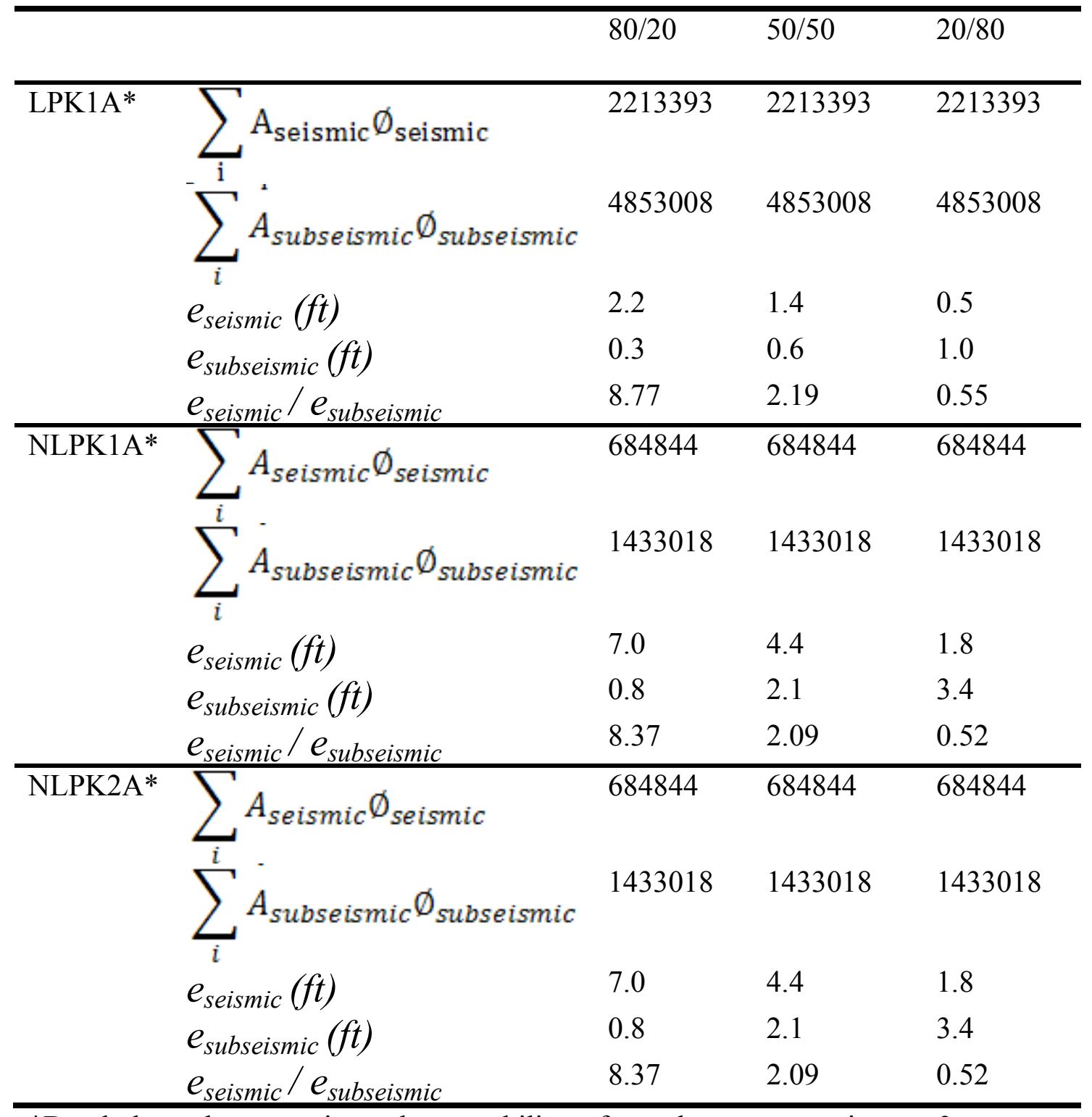

*Depth dependent porosity and permeability refer to data summary in case 3 
Table 5.9 Case 4 simulation summary at the end of simulations (day 2,000)

\begin{tabular}{llll}
\hline & $\begin{array}{l}\text { LPK1A } \\
\text { Recovery }\end{array}$ & $\begin{array}{l}\text { NLPK1A } \\
\text { Recovery }\end{array}$ & $\begin{array}{l}\text { NLPK2A } \\
\text { Recovery }\end{array}$ \\
\cline { 2 - 4 } $\mathbf{8 0}^{2} \mathbf{2 0}^{*}$ & 0.66 & 0.45 & 0.54 \\
$\mathbf{5 0}^{*} \mathbf{5 0}^{*}$ & 0.60 & 0.38 & 0.49 \\
$\mathbf{2 0} / \mathbf{8 0}^{*}$ & 0.54 & 0.31 & 0.42 \\
\hline$* 80 / 20$ means & $80 \%$ of OOIP is distributed in seismic features and $20 \%$ of OOIP is in subseismic \\
features &
\end{tabular}

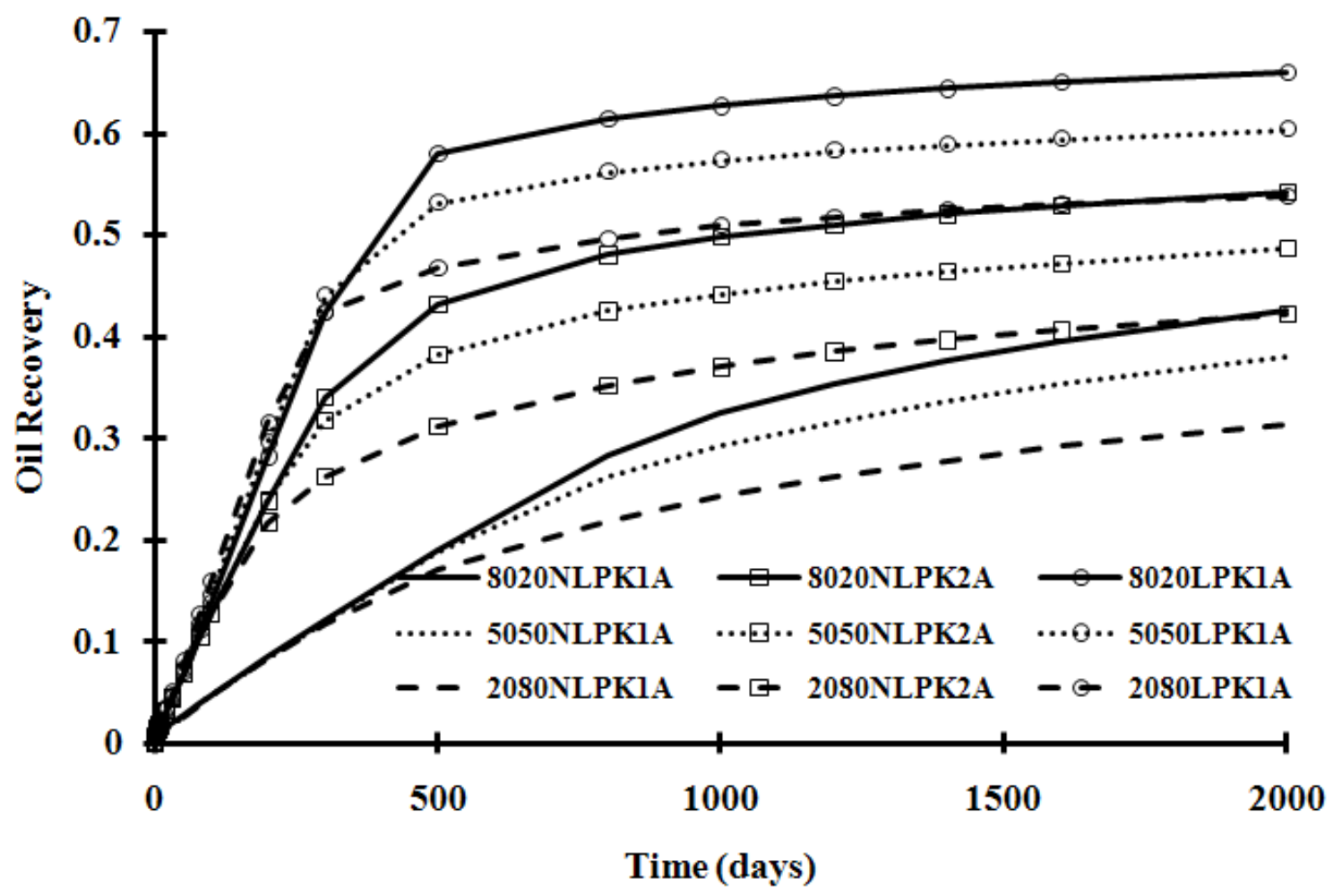

Figure 5.10 Comparisons of oil recovery versus simulation time for case 4 sets of simulations 


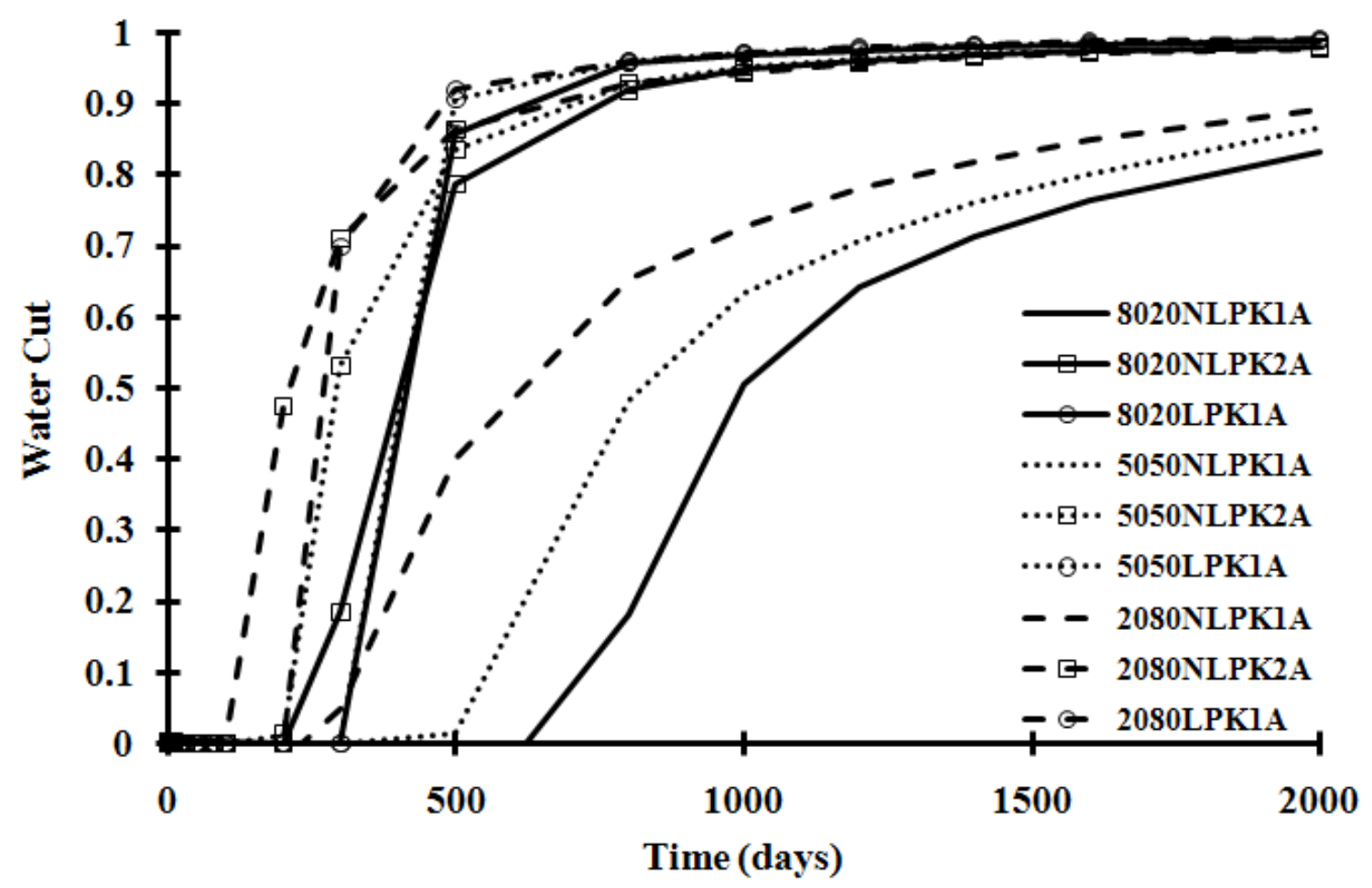

Figure 5.11 Comparisons of water cut versus simulation time for case 4 studies.

breakthrough. It should be noted that this is a reinforcement of the results of the previous

\section{Production Rate Effects with Depth Dependent Reservoir Properties and Proportional OOIP Distributed in Seismic and Subseismic Features}

Excessive water production is an often observed phenomenon from fractured basement reservoirs (Chan et al., 2006). Excess water reduces the artificial lift efficiency and sometimes is believed to cause irreversible damage to oil bearing zones. The postproduction treatment of water, required for environmental reasons, is also very expensive. The understanding of how production rate affects the displacement of oil by water in a basement reservoir system is essential to the operation of these reservoirs.

In this section, total flow rate control was imposed on all the six production wells on the top of two seismic features. Based on the case studies above, two rates were examined: high rate at 712.4 STB/day and low rate at $171.8 \mathrm{STB} /$ day. One of the depth dependent reservoir property models (nonlinear porosity with porosity-permeability correlation 2, "NLPK2A") was chosen 
with three OOIP distribution scenarios in this work. Therefore, six simulations were performed, summarized in Table 5.10.

Figure 5.12 and 5.13 clearly show that simulations with higher total production rate control will result in earlier water breakthrough than the lower rate simulations. For the same controlling rate, simulations with more OOIP distributed in the seismic features offers delayed water breakthrough. This phenomenon makes sense since the supporting water is coming from the bottom of seismic features: more oil originally distributed in the seismic features requires more time to sweep it out. Oil recoveries in case 5 simulation are summarized in Table 5.11.

Two quantitative comparisons are listed below. Comparing different OOIP distributions in subseismic features from $20 \%$, to $50 \%$ to $80 \%$ with same operating rates at the injected PV of 1.0:

1) With the higher operating rate: Recovery decreases from 0.51 , to 0.46 , to 0.40 .

2) With the lower operating rate: Recovery decreases from 0.55 , to 0.49 , to 0.44 .

Table 5.10 Summary of reservoir properties and operating conditions for case 5 sets of simulations.

\begin{tabular}{lclllll}
\hline & $\begin{array}{l}\mathbf{8 0 2 0} \\
\text { HighR }\end{array}$ & $\begin{array}{l}\mathbf{8 0 2 0} \\
\text { LowR }\end{array}$ & $\begin{array}{l}\mathbf{5 0 5 0} \\
\text { HighR }\end{array}$ & $\begin{array}{l}\mathbf{5 0 5 0} \\
\text { LowR }\end{array}$ & $\begin{array}{l}\text { 2080 } \\
\text { HighR }\end{array}$ & $\begin{array}{l}\text { 2080 } \\
\text { LowR }\end{array}$ \\
\hline Porosity & $0.1 \sim 0.46$ & & & & \\
\hline Thickness (ft) & & & & & & \\
(seismic) & 7.01 & 7.01 & 4.38 & 4.38 & 1.75 & 1.75 \\
(subseismic) & 0.84 & 0.84 & 2.10 & 2.10 & 3.35 & 3.35 \\
\hline
\end{tabular}

\section{Permeaiblity (md)}

(seismic \& subseismic) $\quad 748 \sim 123884$

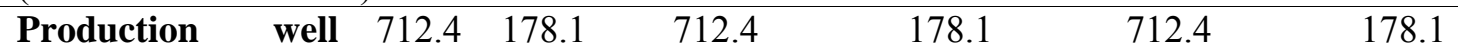

(STB/day)

* "8020" means $80 \%$ OOIP in seismic features and 20\% OOIP in subseismic features, similar interpretation for " 5050 " and " 2080 "

Table 5.11 Case 5 simulation summaries

\begin{tabular}{lllllll}
\hline & $\mathbf{8 0 2 0}$ & $\mathbf{8 0 2 0}$ & $\mathbf{5 0 5 0}$ & $\mathbf{5 0 5 0}$ & $\mathbf{2 0 8 0}$ & 2080 \\
& HighR & LowR & HighR & LowR & HighR & LowR \\
\hline $\begin{array}{l}\text { Oil recovery (at } \\
\text { injected PV of 1.0) }\end{array}$ & 0.51 & 0.55 & 0.46 & 0.49 & 0.40 & 0.44 \\
\hline * Cumulative water production / pore volume & & & & & \\
\hline
\end{tabular}




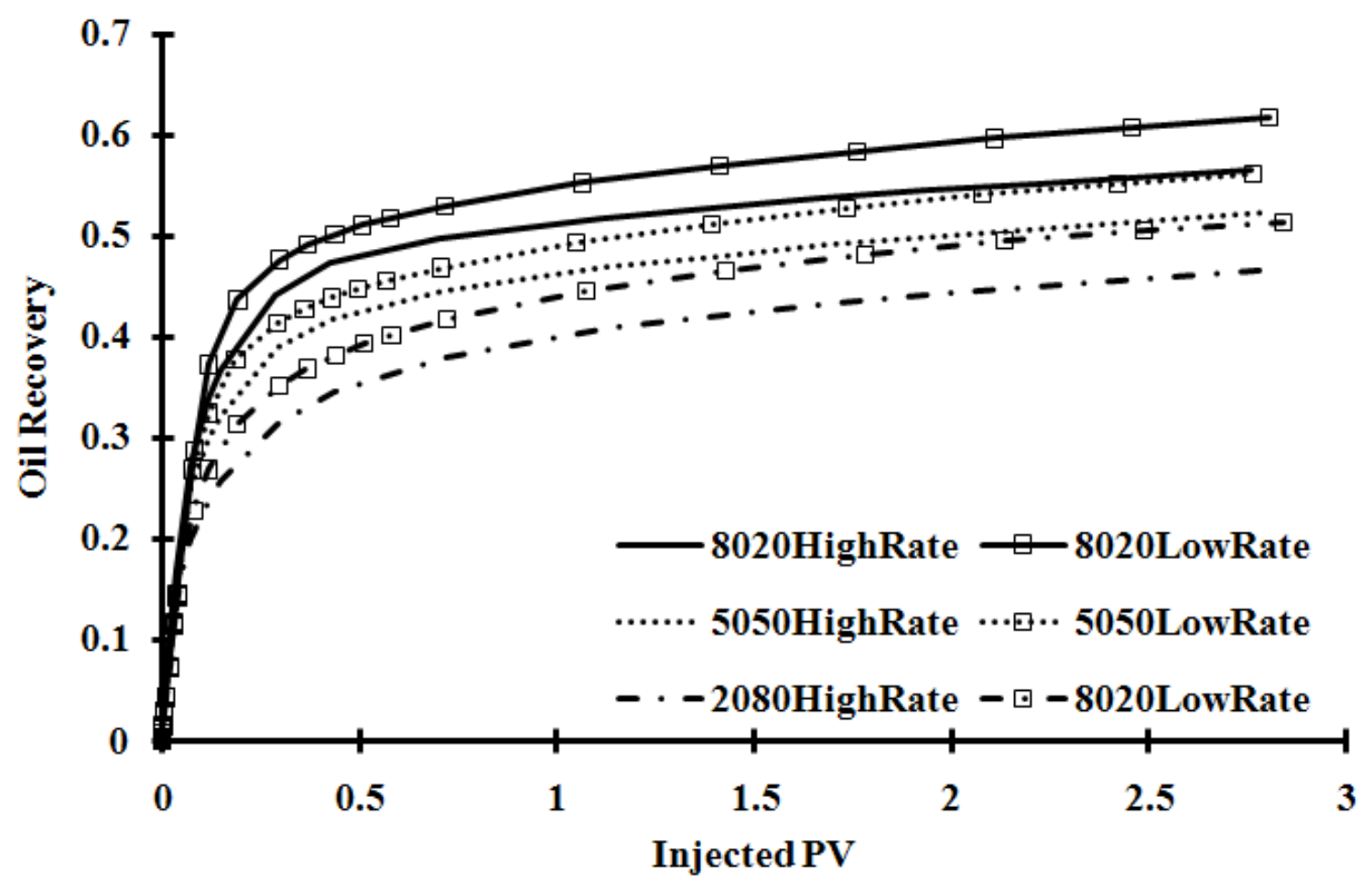

Figure 5.12 Comparisons of oil recoveries in case 5

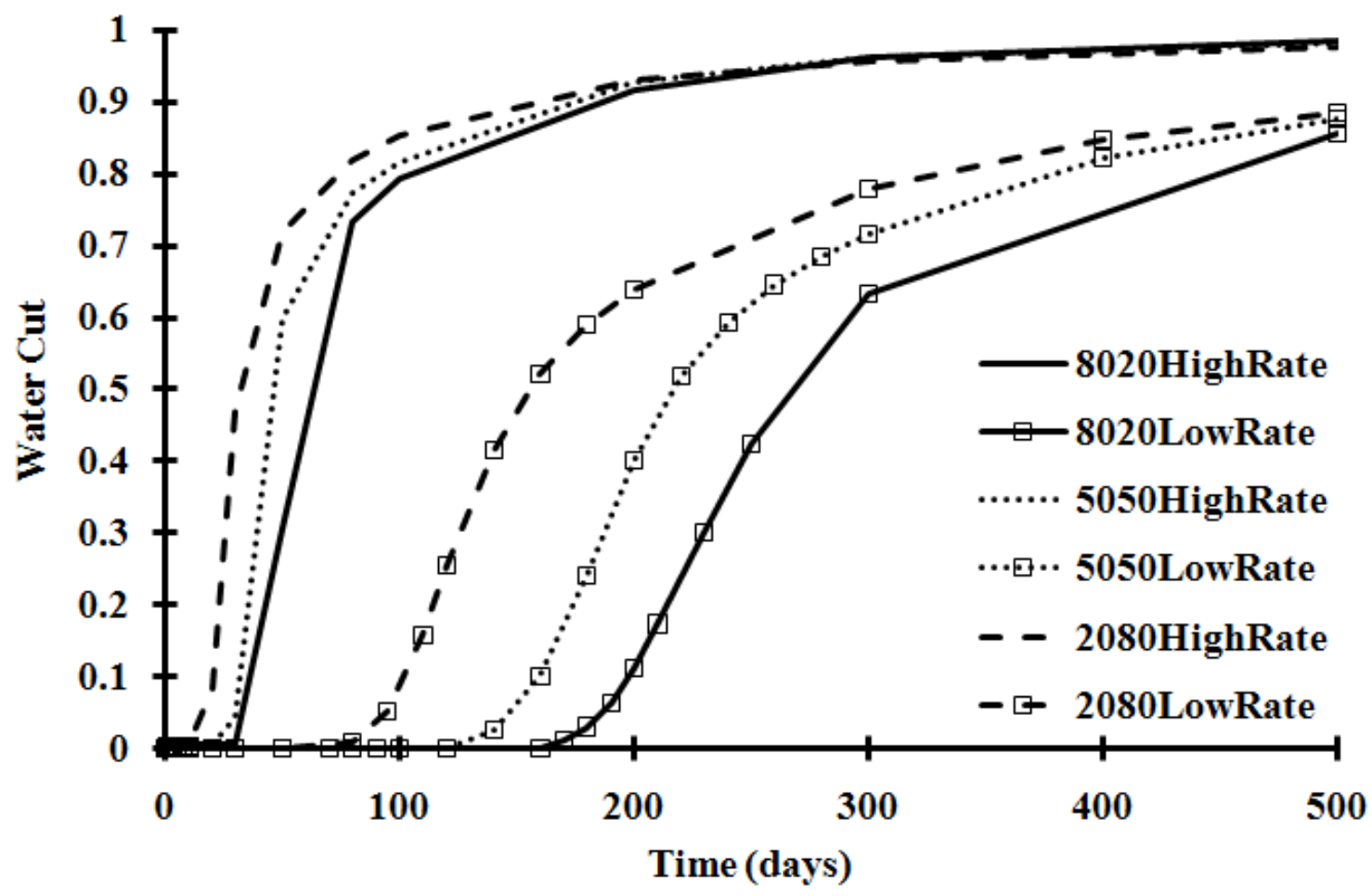

Figure 5.13 Comparisons of water cut in case 5 
Comparing different operating rates with same OOIP distributions:

1) With $20 \%$ OOIP being distributed in subseismic features: Oil recoveries from high rate and low rate are: 0.51 and 0.55 . There is $4 \%$ difference.

2) With $50 \%$ OOIP being distributed at subseismic features: Oil recoveries from high rate and low rate are: 0.46 and 0.49 . There is $3 \%$ difference.

3) While $80 \%$ OOIP being distributed at subseismic features: Oil recoveries from high rate and low rate are: 0.40 and 0.44 . There is $4 \%$ difference.

The above two comparisons show that higher production rate will result in higher recovery by the same time period but lower recovery by the same amount of water being injected. Lower operating rate always results in much delayed water breakthrough than higher operating rate.

Figure 5.14 shows the water cut with oil recovery. For a given recovery, the water cut is always lower when lower rate is used. The absolute values are different, but as more oil is distributed in the subseismic features, the relative recoveries with respect to low and high rates do not change significantly. Given the permeability distribution, $\mathrm{K}$ in the gravity number is fixed, no matter how it is calculated. The only variant appears to be the Darcy velocity which is most significantly impacted by the rate. Hence changing relative distribution of oil does not affect the differences between low and high rate.

Some residual oil saturation snapshots are presented at the end of simulations (day 2,000). As shown in Figure 5.15, the left column represents high production rate studies and the right column represents the low production rate studies; the first row is 20/80 OOIP distributions characterization, then the second and third rows are 50/50 and 80/20 OOIP distribution scenarios. 


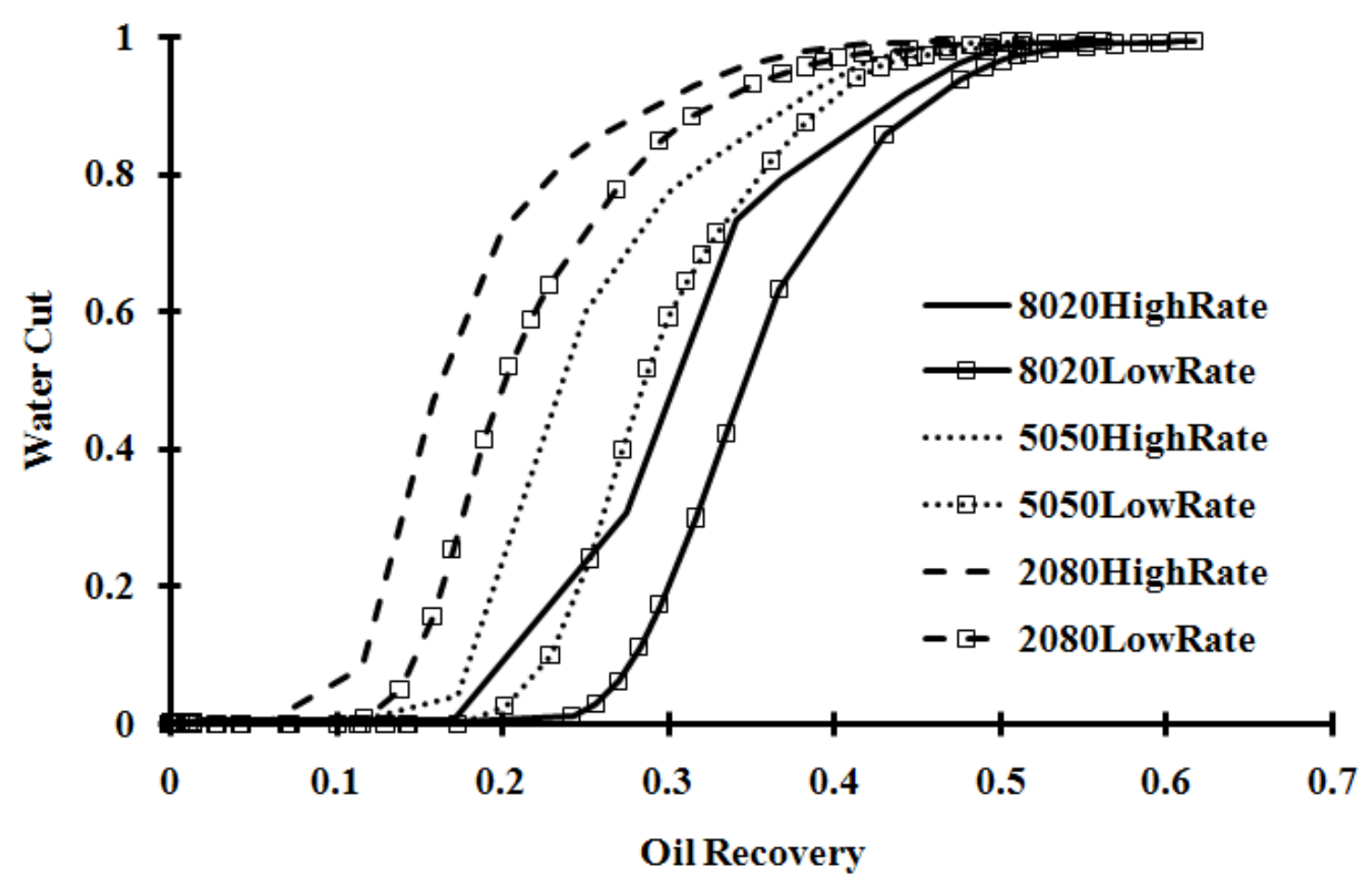

Figure 5.14 Dimensionless analyses of water and oil productions in case 5

Figure 5.15 shows that more residual oil is trapped in subseismic features than in seismic features. During the reservoir characterization, the more OOIP being assigned into subseismic features, the less oil can be flooded out. This explains why the lower subseismic OOIP distribution always has the highest oil recovery among various OOIP distributions.

Observed between columns on each row, high operating rate (left column) always get more oil swept than the low rate operating conditions. The water levels on both seismic and subseismic features are higher in the high operating rate cases. This phenomenon is more obvious in the picture of time dependent oil saturation snapshot comparisons. 

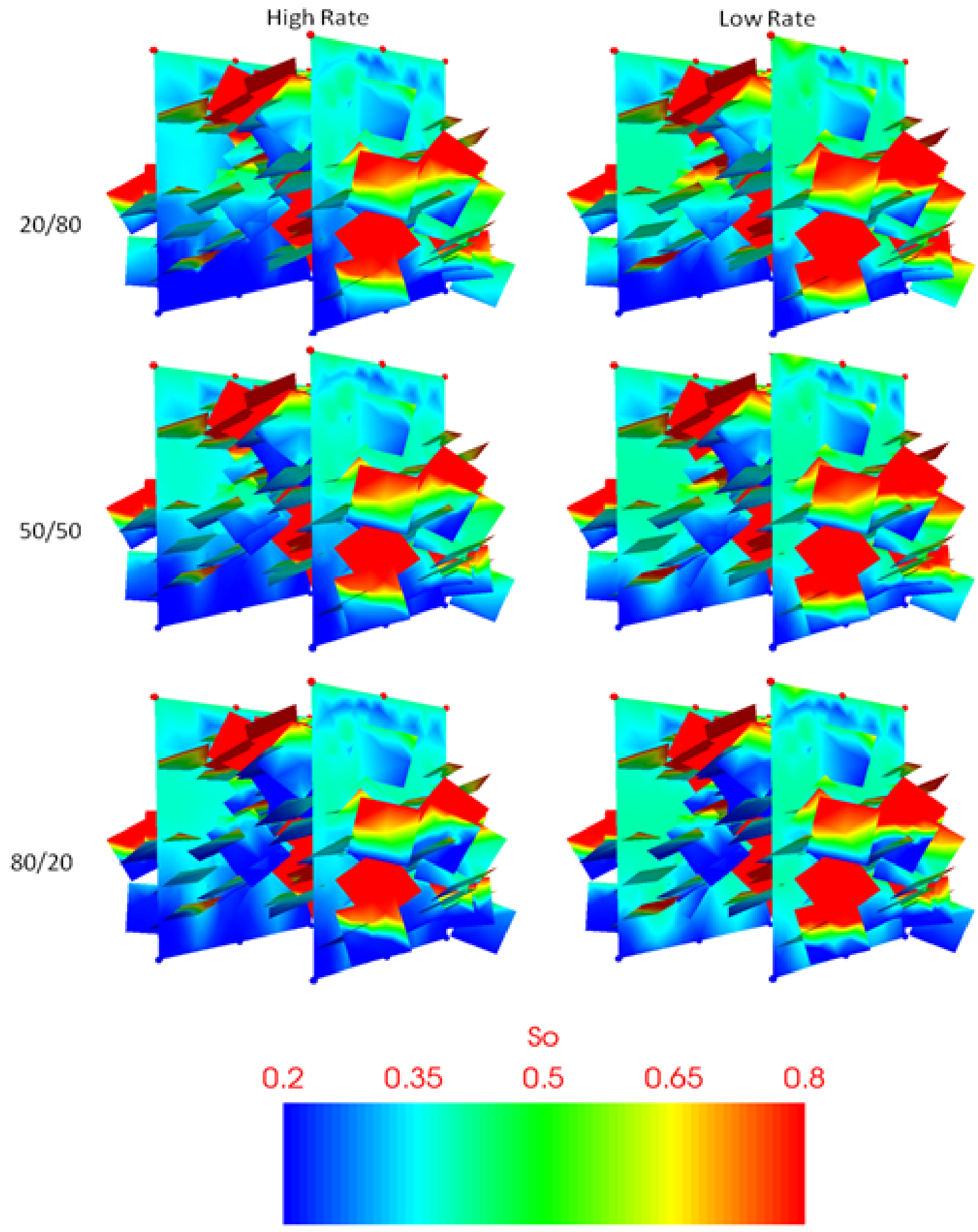

Figure 5.15 Oil saturation comparisons at the day 2,000 (end of simulation) 
The water levels at different OOIP distributions are quite different: $80 / 20$ offers the lowest water level on seismic feature and the highest water level on the subseismic features; 20/80 OOIP distributions has the reverse result compared to $80 / 20$ cases and 50/50 OOIP distribution cases are in the middle between these two. This is due to the OOIP characterization: in order to keep the same OOIP, $80 / 20$ cases have the largest seismic thickness and smallest subseismic thickness and this makes the above phenomenon happen. Another interesting thing that could be observed is by comparing two seismic features on each independent oil saturation file; even though both seismic features have exact boundary and operating conditions, their oil saturations vary considerably. This phenomenon is definitely caused by the existence of subseismic features. The time dependent oil saturation comparisons are shown in Figure 5.16 for the case of 50/50 OOIP distributions. Under the operating condition and assumptions in this case study, two conclusions could be reached through this time dependent comparisons:

1) Seismic feature's oil will be flooded first, then subseismic feature's;

2) For the same time scale, high rate cases will produce more subseismic feature's oil than low rate cases.

\section{Chapter Summary}

This work quantitatively demonstrates hydrocarbons being recovered from a conceptual fractured basement reservoir model with bottom water supporting system on the major seismic features. Different percentages of OOIP were distributed between seismic and subseismic scale features in this reservoir model. Furthermore, various reservoir property models and operating conditions under the same boundary condition 

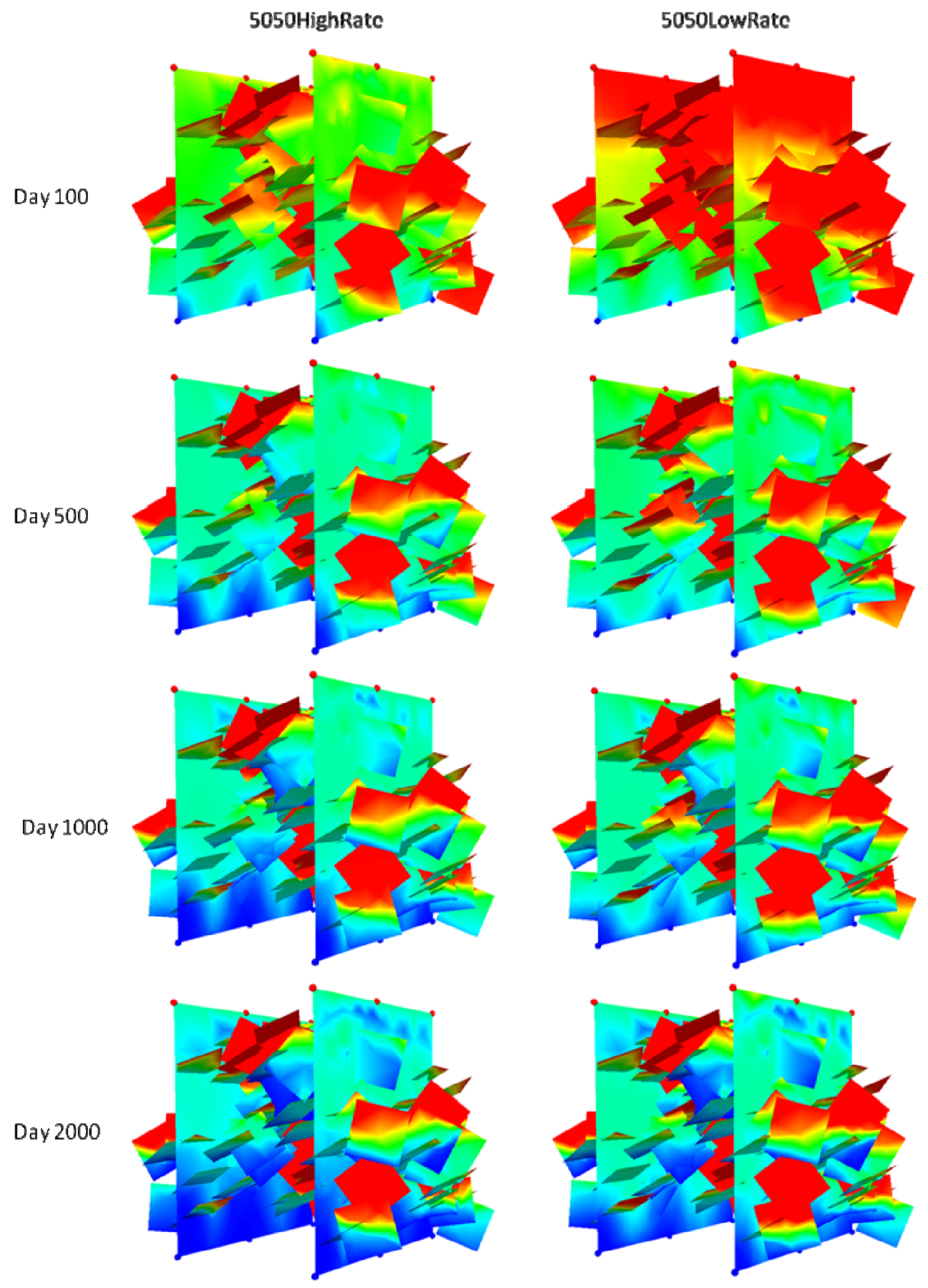
were studied. A new three-dimensional, multiphase, upstream mobility weighted CVFE simulator was used in all simulations. Some conclusions could be reached from this study:

1) CVFE simulator is a very powerful tool to directly simulate multiphase flow in DFN characterized fractured basement reservoirs.

2) If the boundary conditions are ascertained, basement reservoir's recovery factor will be highly dependent on OOIP distributions and reservoir property distributions at both the seismic/subseismic scales. Hence characterization of the basement reservoir is of paramount importance.

3) Reservoir operating conditions will definitely affect basement reservoir behavior. To reach the same oil recovery, lower operating rate is preferred for decreasing water production and early breakthrough.

4) The existence of subseismic scale features does have a big impact on basement reservoir production behavior.

5) The connectivity, orientation, and size distribution of basement features will affect the basement reservoir production behavior. Boundary conditions are a very important consideration to affect oil productions from basement reservoir. Other than bottom water support, side water intrusion on the same reservoir model might lead us to different quantitative results. Qualitatively, the results are expected to be the same.

To understand the basement reservoir's water-oil displacement mechanism deeper, some work could be suggested for future study:

1) Boundary aspect: try different boundary conditions, either declining bottom pressure support or side aquifer support from either seismic features or subseismic scale features. 
2) Geological aspect: try different subseismic feature DFN characterizations with variations of depth dependent sizes distributions, orientations and connectivity. This will expect the heterogeneity from the fracture network geometry may lead to preferred pathways (or oil traps) in the process of water sweeping.

3) Operating aspects: try different operating conditions such as production wells locations with rates or controlling pressures. Some special recovery method also could be studied such as gas injection, $\mathrm{CO} 2 \mathrm{EOR}$, thermal recovery, etc.

4) Reservoir property aspect: try different fluid properties such as PVT, relative permeability, capillary pressure and three-phase (with solution gas involved) model. This is to study the fluid property impacts on the basement hydrocarbon recoveries.

To be considered a valid model of the basement reservoir, all heterogeneity uncertainties have to be evaluated to study the reservoir behavior practically.

\section{Chapter Nomenclature}

$\begin{array}{lll}A & = & \text { area, } \mathrm{ft}^{2} \\ B & = & \text { formation volume factor } \\ \mathbf{c} & = & \text { compressibility, } 1 / \text { pisa } \\ e & = & \text { fracture/fault storage thickness, } \mathrm{ft} \\ g & = & \text { acceleration of gravity, } \mathrm{ft} / \mathrm{sec}^{2} \\ \mathbf{k} & = & \text { permeability tensor, } \mathrm{md} \\ k_{r} & = & \text { relative permeability } \\ n & = & \text { number of discretized fracture elements } \\ N_{G} & = & \text { gravity number } \\ o & = & \text { oil phase }\end{array}$




$\begin{array}{lll}p & = & \text { fluid pressure, psi } \\ q & = & \text { flux vector } \\ R & = & \text { oil recovery } \\ S & = & \text { phase saturation } \\ u & = & \text { velocity, ft/day } \\ w & = & \text { water phase } \\ W C & = & \text { water cut } \\ W P & = & \text { water production, pore volume } \\ \emptyset & = & \text { porosity } \\ \rho & = & \text { density, } \mathrm{lbm} / \mathrm{ft}^{3} \\ \mu & = & \text { viscosity, } \mathrm{cp}\end{array}$

\section{Chapter Bibliography}

Araujo, H., Lacentre, P., Zapata, T., Del Monte, A., Dzelalija, F., Repsol-YPF; Gilman, J., Meng, H., Kazemi, H., Ozkan, E., "Dynamic Behavoir of Discrete Fracture Network (DFN) Models," paper SPE 91940 (2004)

Basquet, R., Cohen, C.E. and Bourbiaux, B., "Fracture Flow Property Identification: An Optimized Implementation of Discrete Fracture Network Models," paper SPE 93748 (2005)

Behrenbruch, P., Du, P.Q., "The Significance of Large Variations in Oil Properties of the Dai Hung Field, Vietnam,” SPE 29302, (1995)

Chan, K., Lam D.D., Ivanov, A., Apisitsareekul, K., Hai, L.V., Nghi, N.C., Hung, V.Q., and Lang. L.D, "Production Improvement Water Shutoff for White Tiger Field," SPE 103329 (2006)

Dershowitz, B., LaPointe, P., Eiben, T., Wei, L., "Integration of Discrete Feature Network Methods with Conventional Simulator Approaches," paper SPE 49069 (1998)

Fu, Yao, "Multiphase Control Volume Finite Element Simulations of Fractured Reservoirs," Ph.D Dissertation, University of Utah, Salt Lake City (2007)

Li, B., Guttormsen, J., Hoi, T.V., Duc, N.V., "Characterizing Permeability for the Fractured Basement Reservoirs," SPE 88478, (2004) 
Pooladi-Darvish, M. and Firoozabadi, A., "Experiments and Modelling of Water Injection in Water-wet Fractured Porous Media," J. Can. Pet. Tech., vol. 39, no. 3 (2000)

Ronald A. Nelson, "Geologic Analysis of Naturally Fractured Reservoirs," Gulf Professional Publishing, second edition, (2001)

Sibbit, A.M., "Quantifying Porosity and Estimating Permeability from Well Logs in Fractured Basement Reservoirs," SPE 30157 (1995)

Will, R., Archer, R., Dershowitz, B., "Integration of Seismic Anisotropy and Reservoir-Performance Data for Characterization of Naturally Fractured Reservoirs Using Discrete-Feature-Network Models," SPE $84412(2005)$

Yang, Yi-kun, "Finite-element Multiphase Flow Simulation," Ph.D Dissertation, University of Utah, Salt Lake City, UT (2003) 


\section{Chapter 6. Simulation of Complex Matrix-Fracture Systems}

\section{Table of Contents}

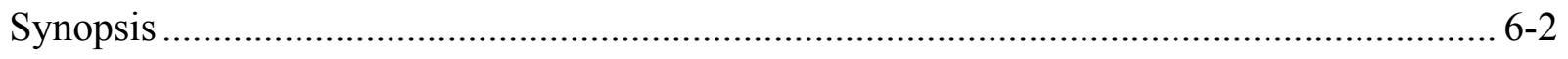

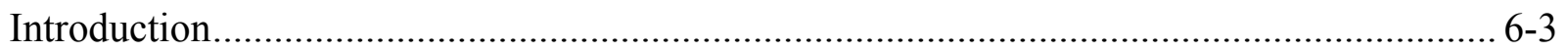

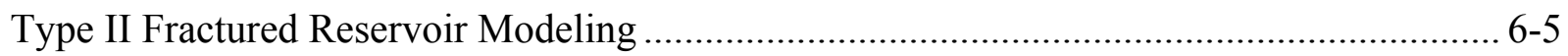

CVFE Simulator Verification with Pooladi-Davish and Firoozabadi's Fracture Water Level

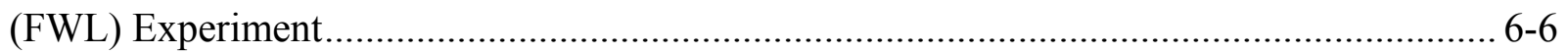

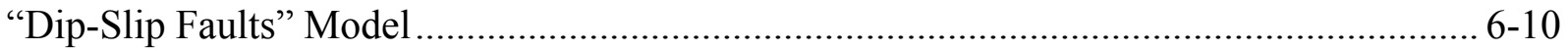

Outcrop to Simulation: "Teasdale Fault" Line Drive Scenario: Heterogeneous Matrix with

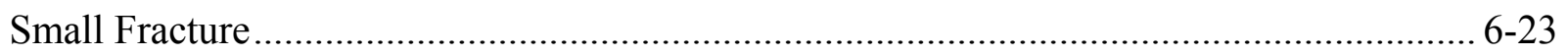

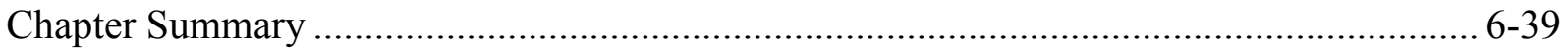

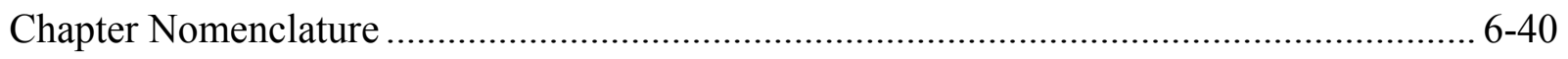

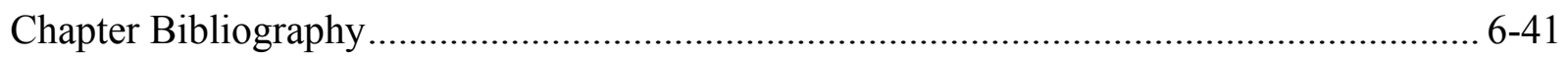




\section{Synopsis}

A new fully-implicit, three-dimensional (3-D), three-phase, discrete fault/fracture with matrix, black-oil simulator provides new insight and understanding of oil production from reservoirs in fractured, medium-permeability sandstone or carbonate formation. Results obtained with a controlled volume finite element (CVFE) method compare favorably to the lab scale core experiment. One quarter of a lab core experimental sample ( 18.75 by 18.75 by $121.92 \mathrm{~cm})$ with five orthogonal fractures was modeled by discrete fracture network (DFN) in this study. At the beginning of this chapter, this model was used to verify the new simulator. The results on this core scale modeling are highly matched lab results at low flow rate. In the high flow rate region, the simulation results reflected a slightly delayed water breakthrough but predicted excellent final recovery fraction with the experiment. Then, dip-slip fault and strike-slip fault geological models were constructed and studied for various purposes of fracture network impact on Type II reservoirs. By these studies, the following aspects of Type II reservoirs were quantitatively evaluated: understanding the importance of a fault that separates and connects two lobes of a fractured reservoir, line drive scenarios on a heterogeneous matrix with fractures, and existence of ultrahigh permeability faults between injector and producer on a heterogeneous system. This work demonstrated that the CVFE method provides a much-improved ability to represent complex fracture/fault geometries in porous formations underground and that it has a strong capability to handle the multiphase fluid flow problems. These advantages are important in helping understand the uncertainties caused by fracture network existence when attempting to develop innovative approaches to reservoir management. 


\section{Introduction}

Naturally fractured reservoirs make up a large and increasing percentage of the world's oil/gas reserves. Generally, naturally fractured reservoirs could be classified in four types (Nelson, 2001) from the fractures' positive contributions to the overall reservoir quality. Type II fractured system is the largest fractured reservoir type worldwide. In this reservoir type fractures provide the essential reservoir permeability and matrix supplies any significant porosity or storage. An early knowledge of fracture/matrix interaction is extremely important to determine whether the matrix porosity can be drained by the fracture system. The recovery from reservoirs where fractures dominate permeability is often a fraction of the resource recovered from conventional reservoirs in which matrix permeability dominates. The lower recovery and higher risks relate to the difficulty of forecasting how various completion placements, water flood patterns, and tertiary recovery processes will actually perform in fractured reservoirs. A reduction in risk and an improvement in understanding of reservoir behavior will lead to enhanced profitability from under-exploited fractured fields. Fractures do more than simply increase reservoir permeability. Fundamentally, fractures alter reservoir connectivity and heterogeneity. A precise characterized fracture network model will be the key to improving oil and gas recovery in naturally fractured reservoirs. Identifying, characterizing, and mapping the fracture network in terms of the dominant flow paths, aperture, length, height, connectivity, conductivity, and frequency distribution are crucial for optimal reservoir management. However, it is well known that the accurate simulation of multiphase fluid flow in this reservoir type remains a significant challenge.

Dual-porosity modeling is the most popular simulation technique for Type II fractured reservoir flow predictions. This model was first introduced to the petroleum industry by Warren and Root 
(1963), and methods for simulating dual-porosity systems have been proposed by several authors (Kleppe and Morse, 1974; Yamamoto et al., 1971; Kazemi et al., 1976; Rossen, 1977; Dean and Lo, 1988; Fung et al., 1991; and others). If the matrix blocks are linked only through the fracture system, this could be treated conventionally as a dual porosity single-permeability system, since fluid flow though the reservoir takes place only in the fracture network with the matrix blocks acting as sources. If there is the possibility of flow directly between neighboring matrix blocks, this is conventionally considered to be a dual porosity dual permeability system. The dual porosity system is represented by two different continua, one representing the porous matrix and the other representing the fractures. Fluid flow is primarily through the high permeability, low porosity fractures surrounding individual matrix elements. The matrix blocks contain the majority of the reservoir volume and act as sources or sinks to the fractures. A transfer function describes the mass transfer between the matrix and fracture continua. This core part of transfer function is represented by a shape factor. The whole picture of the dual-porosity model is that the geological and flow complexity are reduced to a single parameter - the shape factor and the shape factor might be too ideal to represent fluid flow in such a heterogeneous environment (Gong et al., 2006). The model is also limited to the sugar cube representation of fractured media. The transfer function between the fracture and the matrix may not be properly described when gravity and viscous effects are involved. This approach also assumes that the media have dense closely connected fractured networks and may not be very accurate when treating only a few fractures.

The DFN (Dershowitz et al., 1979; Basquet et al., 2005; Araujo, et al., 2004) approach models the geometry of the fracture network explicitly and provides a realistic way of modeling fractured reservoir performance. However, these DFN descriptions are generally too detailed to 
be simulated by conventional reservoir simulators. Some research has been done by connecting DFN with the dual-porosity model for the case of single-phase flow by Dershowitz (1998). This approach is limited with single-phase flow, but no accurate multiphase flow study has been reported.

In this study, a flux-based upstream-weighted three-dimensional, two-phase (oil, water) black-oil CVFE simulator was developed and employed (Yang, 2003; Fu et al., 2005). This CVFE formulation was validated and numerically verified through the indexing method (Chapter 3 ) and the manufactured solution method (Yang, 2003; Fu, 2007). This CVFE formulation's rotatable permeability is good at tensor handling and makes possible the direct accurate simulation of the DFN modeled fractured reservoir.

At the beginning of the study, the simulator was verified with the core scale lab experiment on high/low velocity scenarios. Then several large-scale Type II fractured reservoir models were studied by this simulator. The impact of the fractured network's existence on the Type II fractured reservoir with multiphase (two-phase or three-phase with compressibility, capillary pressure and gravity considered) flow were studied with this new technique. It showed that this is a powerful tool in helping to understand the role of fractures for multiphase flows.

\section{Type II Fractured Reservoir Modeling}

The explicit treatment of fractures and faults is detailed in Kim and Deo (2000) and Yang (2003). The fluid potential and saturation values are defined on the vertices of the tetrahedron for the rock matrix calculations. The tetrahedron and the associated control volumes are shown in Figure 6.1.

For type II fractured reservoir simulations, the fluid potential value in the tetrahedron is interpolated using the interpolation functions. The fluid saturation value is calculated for each 
control volume from the solution of the residual equations, which are the fully discretized partial differential equations. For detailed numerical methods refer to Fu et al. (2005).

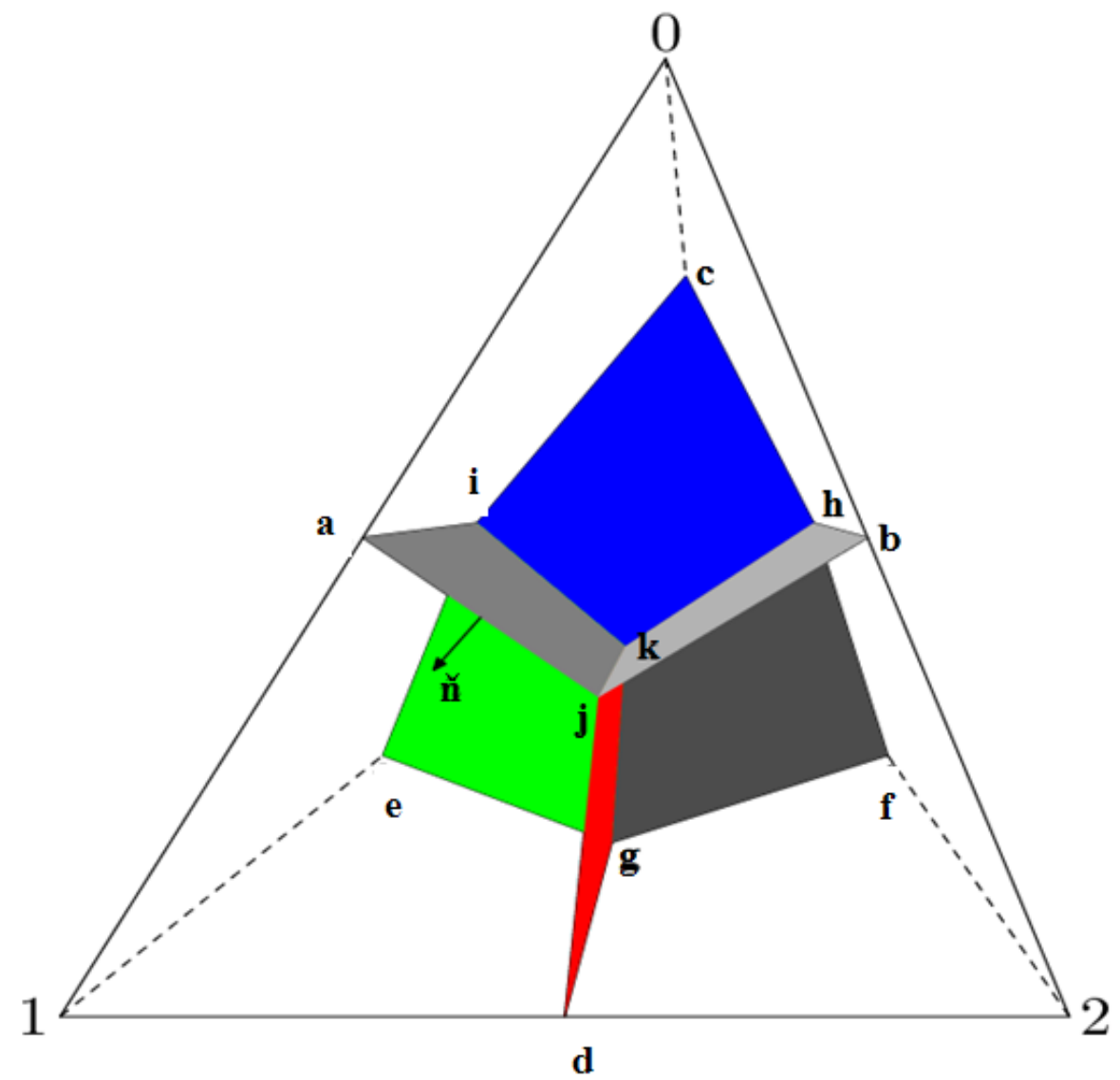

Figure 6.1 A tetrahedral element with associated control volumes

\section{CVFE Simulator Verification with Pooladi-Davish and Firoozabadi's Fracture Water Level (FWL) Experiment}

A reported experiment by Pooladi-Davish and Firoozabadi (2000) was used to verify the Type II CVFE simulator. Montegudo et al. (2004) verified this experiment through the simulation approach with good agreement. Their experiment consisted of flooding water over a type II matrix-fracture apparatus. The apparatus is made by assembling 12 matrix blocks as shown in Figure 6.2. 
Water was injected with different rates from the bottom of the apparatus to expel the $\mathrm{nC} 10$ which initially filled in all the fractures and matrix blocks. They reported the oil recovery versus the pore volume injected at different rates.
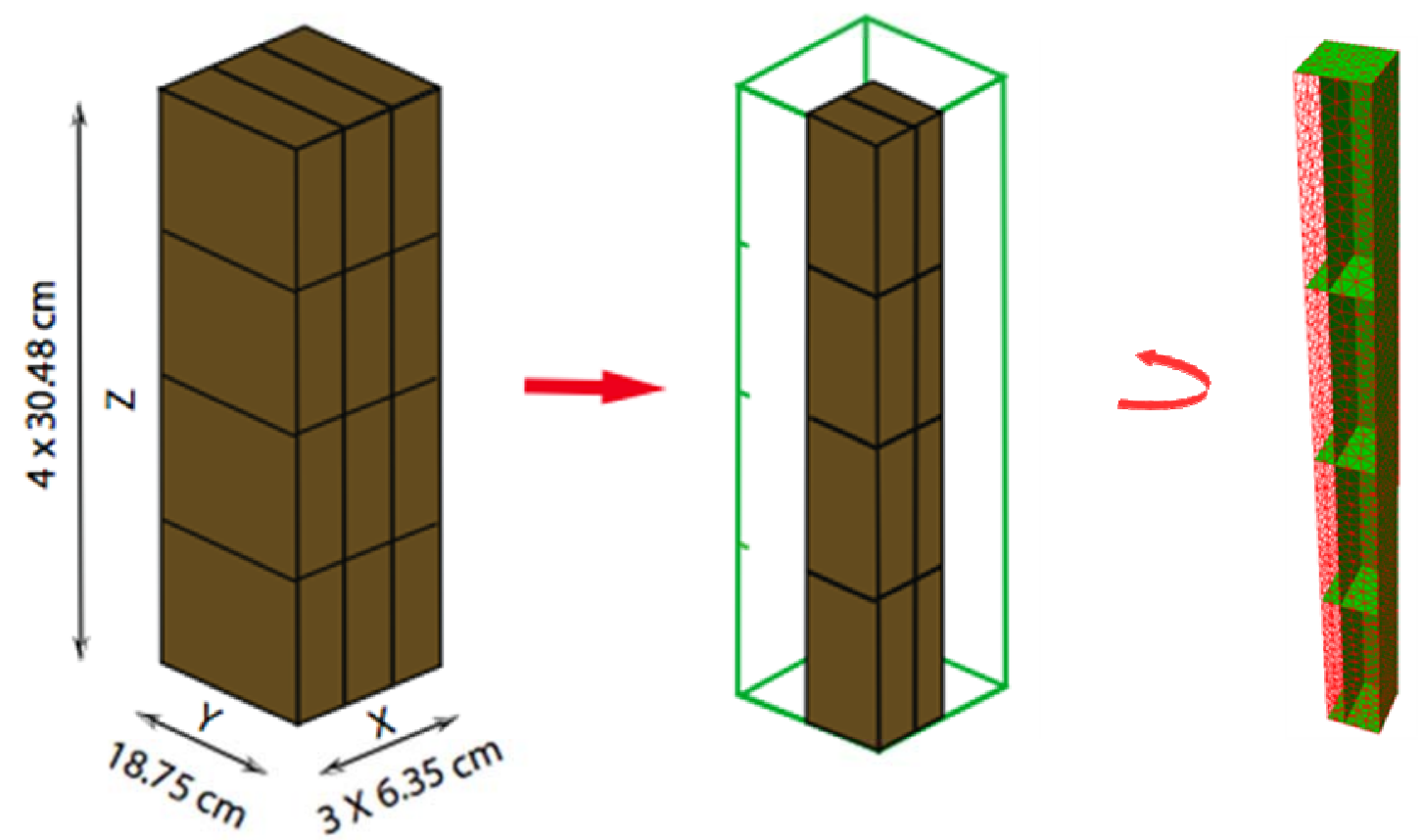

Figure 6.2 Rock assemblies of the stacked blocks and the domain used in the modeling

Considering the symmetry feature of the problem, only a quarter of the domain was adopted to be simulated (as shown above) in this CVFE simulator verification. The domain was meshed by CUBIT, a finite-element mesh generator developed by Sandia National Laboratories. An isothermal black-oil model was used and the critical input parameters for this modeling are summarized in Table 6.1:

The analytical curves are used to calculate relative permeabilities Equations (6-1) and (6-2). 
Table 6.1 Summary of critical data for modeling Pooladi-Davish and Firoozabadi's fracture water level experiment

\begin{tabular}{|c|c|c|}
\hline Simulator & CVFE & \\
\hline \multicolumn{3}{|l|}{ Grid information } \\
\hline $\mathbf{N}_{\mathrm{t}}$ & $2374,6741,9488$ & \\
\hline \multirow{3}{*}{$\begin{array}{l}\text { Fluid property } \\
\mu(\mathrm{cP}) \\
\gamma\left(\mathrm{lbf} / \mathrm{ft}^{3}\right)\end{array}$} & Water & oil \\
\hline & 1.0 & 0.94 \\
\hline & 59.28 & 49.92 \\
\hline \multicolumn{3}{|l|}{ Rock property } \\
\hline$\Phi(\mathrm{m}, \mathrm{f})$ & $0.315,1.0$ & \\
\hline $\mathrm{k}(\mathrm{m}, \mathrm{f})(\mathrm{md})$ & $2.48,846.37$ & \\
\hline \multicolumn{3}{|l|}{ Rock-fluid data } \\
\hline $\mathrm{S}_{\text {or }}$ & 0.35 & \\
\hline \multicolumn{3}{|l|}{ Fracture } \\
\hline $\mathrm{Pc}_{\mathrm{wo}}(\mathrm{psi})$ & 0.0 & \\
\hline $\mathrm{Kr}_{\mathrm{wo}}, \mathrm{n}_{\mathrm{w}}$ & $1.0,1.0$ & \\
\hline $\mathrm{Kr}_{\mathrm{no}}, \mathrm{n}_{\mathrm{o}}$ & $1.0,1.0$ & \\
\hline \multicolumn{3}{|l|}{ Matrix } \\
\hline $\mathrm{Pc}_{\mathrm{wo}}(\mathrm{psi})$ & $-0.1156 \ln \left(\mathrm{S}_{\mathrm{o}}\right)$ & \\
\hline $\mathrm{Kr}_{\mathrm{wo}}, \mathrm{n}_{\mathrm{w}}$ & $0.2,4.0$ & \\
\hline $\mathrm{Kr}_{\mathrm{no}}, \mathrm{n}_{\mathrm{o}}$ & $0.8,2.0$ & \\
\hline \multicolumn{3}{|l|}{ Initial conditions } \\
\hline $\mathrm{P}(\mathrm{psi})$ & 14.7 & \\
\hline $\mathrm{S}_{\mathrm{w}}$ & 0.0 & \\
\hline \multicolumn{3}{|c|}{ Boundary conditions } \\
\hline & Nonflow boundary conditions & \\
\hline \multicolumn{3}{|l|}{ Well conditions } \\
\hline Injection & Water rate $(\mathrm{PV} / \mathrm{hr})$ & $0.011,0.160$ \\
\hline Production & Total liquid rate (PV/hr) & $0.011,0.160$ \\
\hline
\end{tabular}

$$
\begin{aligned}
& k w_{W F}=k x_{W O} g_{o}^{n_{W}} \\
& k r_{\rho}=k r_{n \rho}\left(1-\xi_{\rho}\right)^{n_{\sigma}}
\end{aligned}
$$

where

$S_{\mathrm{Q}}=\frac{S_{\mathrm{W}}}{1-S_{\mathrm{QP}}}$

The oil recovery results from numerical simulation and experiment are compared with the water injection rate of 0.011 and $0.160 \mathrm{PV} / \mathrm{hr}$ as shown in Figure 6.3. The numerical model simulated by the CVFE simulator predicted slightly lower ultimate oil recovery with both high and low injection rates. At a low injection rate, the CVFE simulator almost predicted the exact the same 
water breakthrough as the Pooladi-Davish and Firoozabadi (2004) experiment. However, with a high injection rate, water relative permeability assigned for the fluid flow with fractures. The capillary force effects might need to be considered in the main fractures during the modeling of this process. Another possible reason for this effect is the fracture permeability set up in DFN modeling. This model was very small and thin compared with the reservoir scale system; also, it was hard to check how many of the system errors might have been generated from the experiment. The fracture permeability might be set slightly lower in the numerical model. Then the permeability becomes essential in the high rate case. At a high injection rate, the breakthrough was dominated by the displacement in the fractures; the process of water imbibitions to drain the oil out of the matrix was comparably slower than the process acting on the fractures. Therefore, an earlier breakthrough compared with the low injection rate case was expected and shown from both numerical simulations and the experiments.

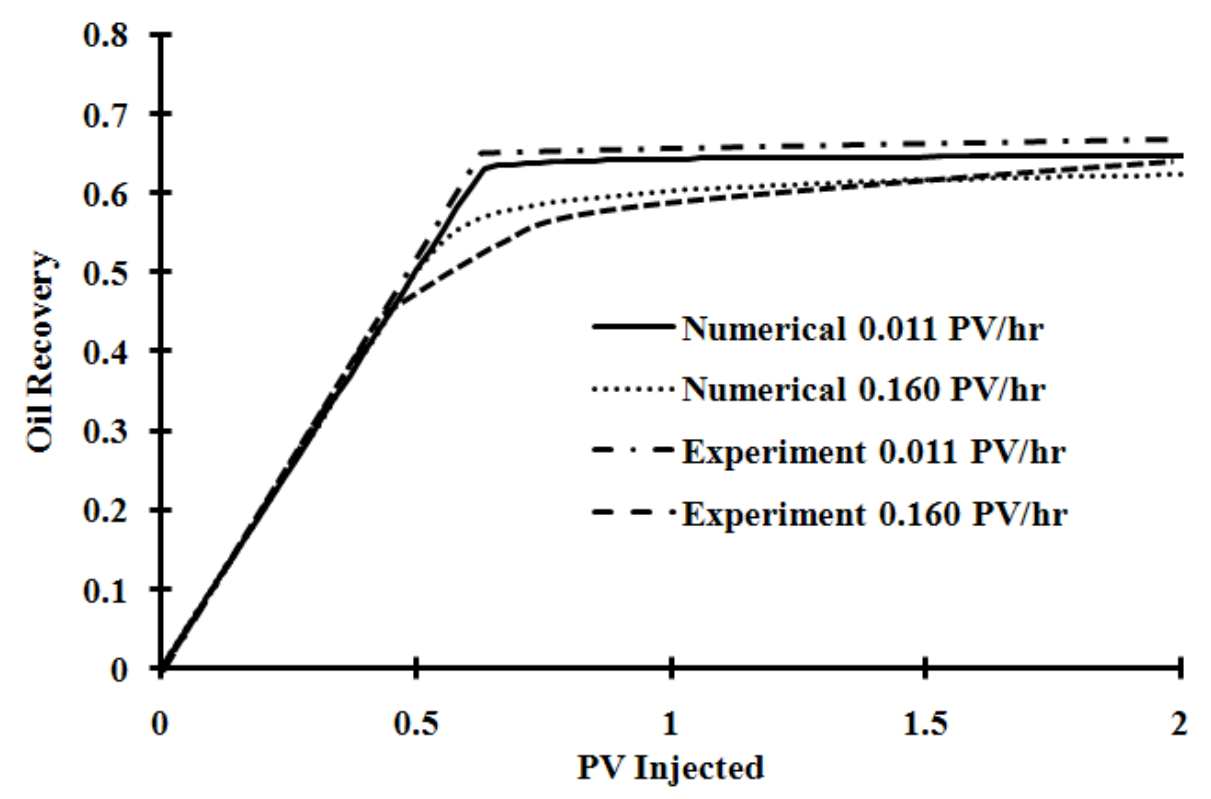

Figure 6.3 Oil recovery comparisons between experiment results and simulation results on low water flooding rate $(0.011 \mathrm{PV} / \mathrm{hr})$ and high water flooding rate $(0.160 \mathrm{PV} / \mathrm{hr})$ breakthrough was delayed in the numerical simulation. This effect might be caused by the unit 
To check if the numerical model converged on the grid sizes, this domain had been meshed into three different finite volume resolutions. The meshed elements numbers were 2374, 6741 and 9488. At the injection rate of $0.160 \mathrm{PV} / \mathrm{hr}$, these three discretized domains were simulated and the oil recoveries are shown in Figure 6.4.

The results showed that a very small discrepancy could be observed at these three grid resolutions. These simulation results indicate that the CVFE simulator can achieve good convergence at different resolution scales and can match experiment results. This suggested that the CVFE simulator could be numerically applied to study complex Type II system.

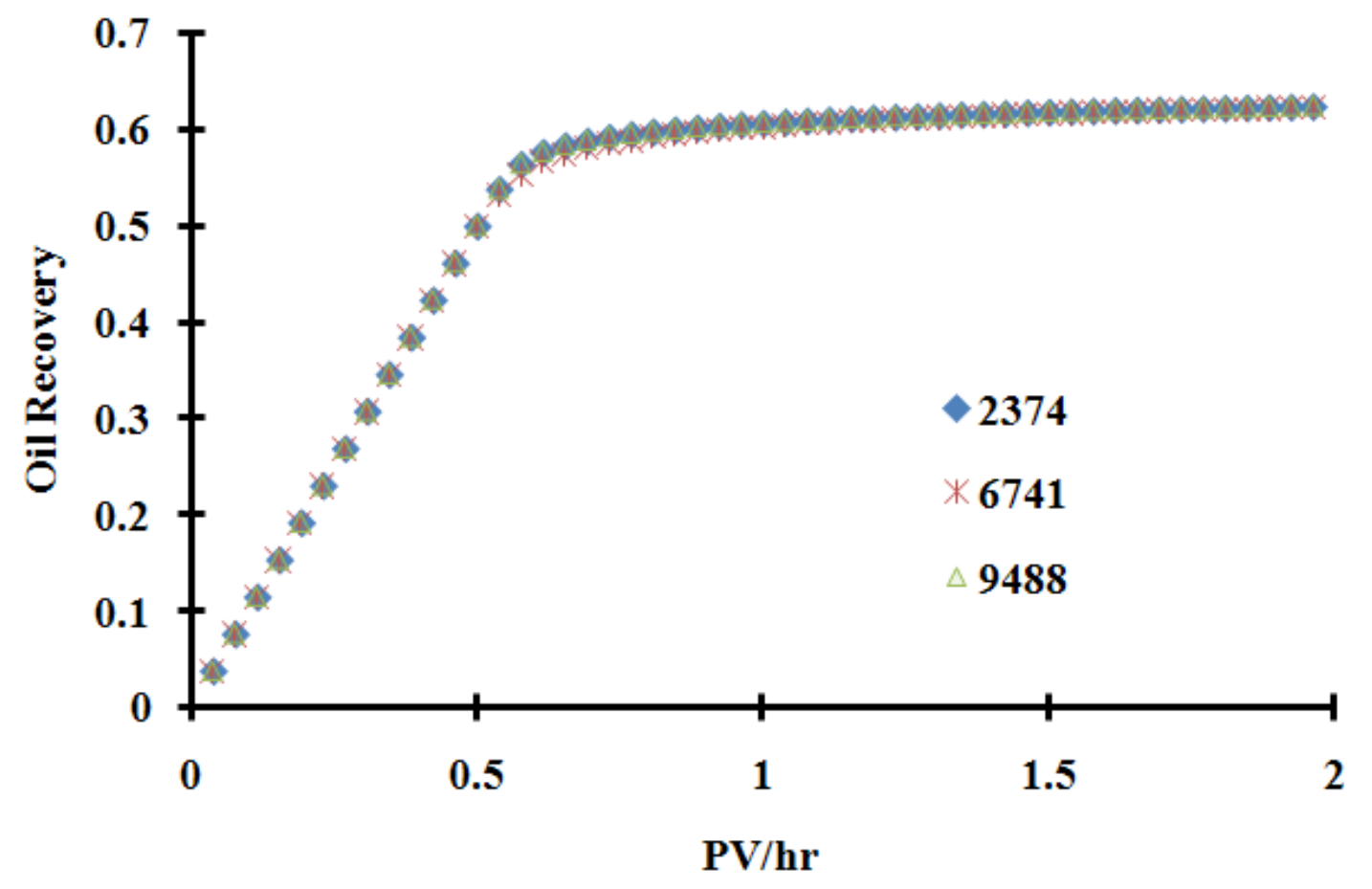

Figure 6.4 Oil recovery comparisons on different finite volume refinement with $0.160 \mathrm{PV} / \mathrm{hr}$ water injection rate

\section{“Dip-Slip Faults” Model}

A Dip-Slip Fault separates and connects two lobes of a fractured reservoir. 
A dip-slip fault is a fault whose main sense of movement (or slip) on the fault plane is vertical. Geologically, dip-slip faults can be classified as "reverse" and "normal." A normal fault occurs when the crust is extended. Alternatively such a fault can be called an extensional fault. The hanging wall moves downward, relative to the footwall. A reverse fault is the opposite of a normal fault-the hanging wall moves up relative to the footwall. Reverse faults are indicate shortening of the crust. The dip of a reverse fault is relatively steep. The angle might be greater than $45^{\circ}$. Normal and reverse dip-slip faults are shown in Figure 6.5.

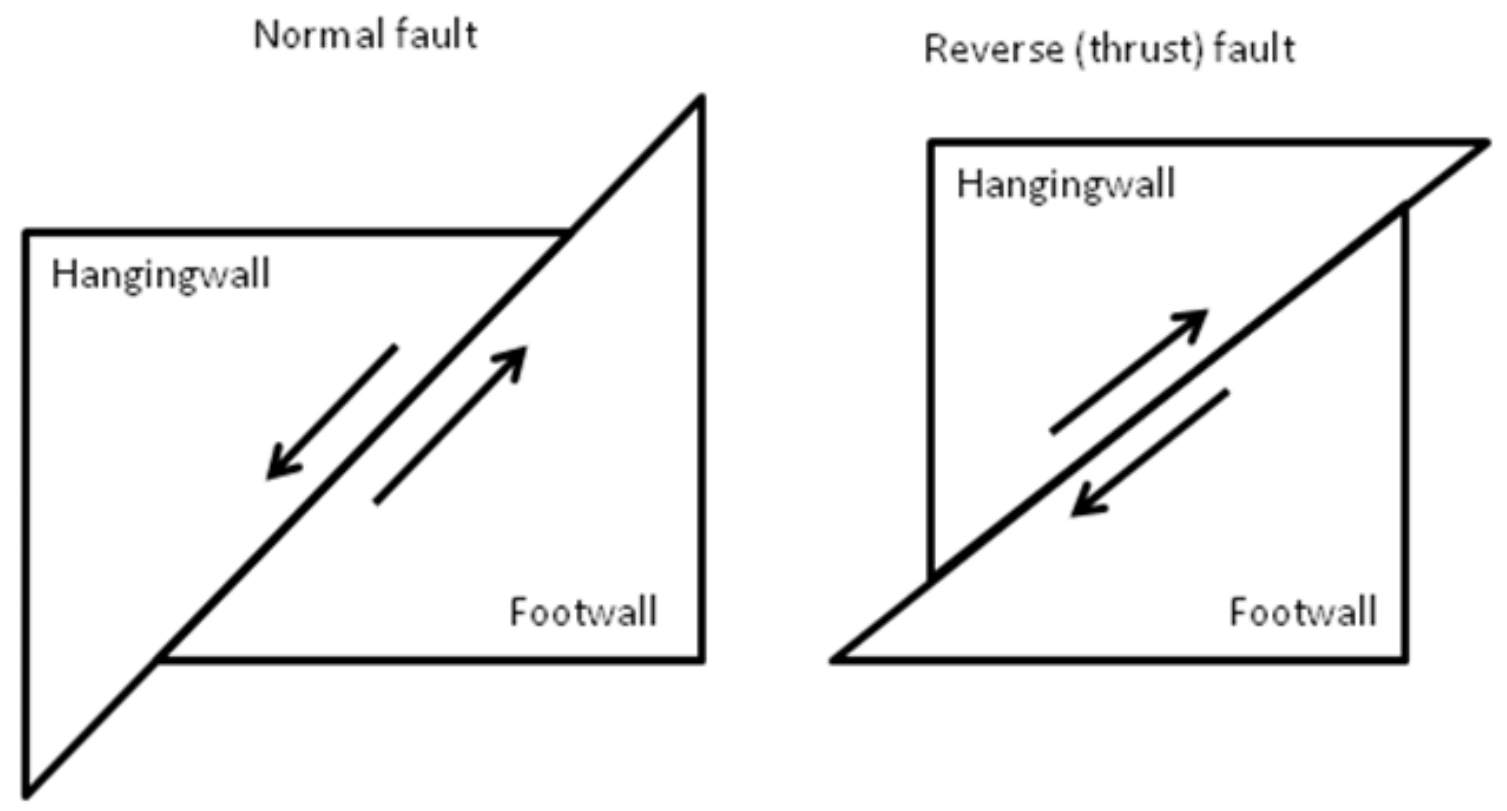

Figure 6.5 Cross-sectional illustrations of normal and reverse dip-slip faults

In this section, a conceptual model of two type II fractured reservoirs connected by a normal fault was constructed and studied. The basic assumption in this model was that in approaching to the normal fault, the intensity of small fractures in the matrix increases, and the matrix porosity and permeability increases as well. Two scenarios were studied in order to compare the normal fault impact on the oil productions in this type of reservoir: very permeable "tunnel" (assigned 10,000 md) and not very permeable "barrier" (assigned $100 \mathrm{md}$ ). There were six wells: two 
wells on the bottom corners of the upper reservoirs (refer to Figure 6.6); two producers on the bottom corners of the dip-slip fault and two producers on the bottom corners of the lower reservoir. The phase model was initially set as three-phase: oil, water and solution gas. For more details regarding reservoir model properties, refer to the data summary table in Figure 6.6 and Figure 6.7.

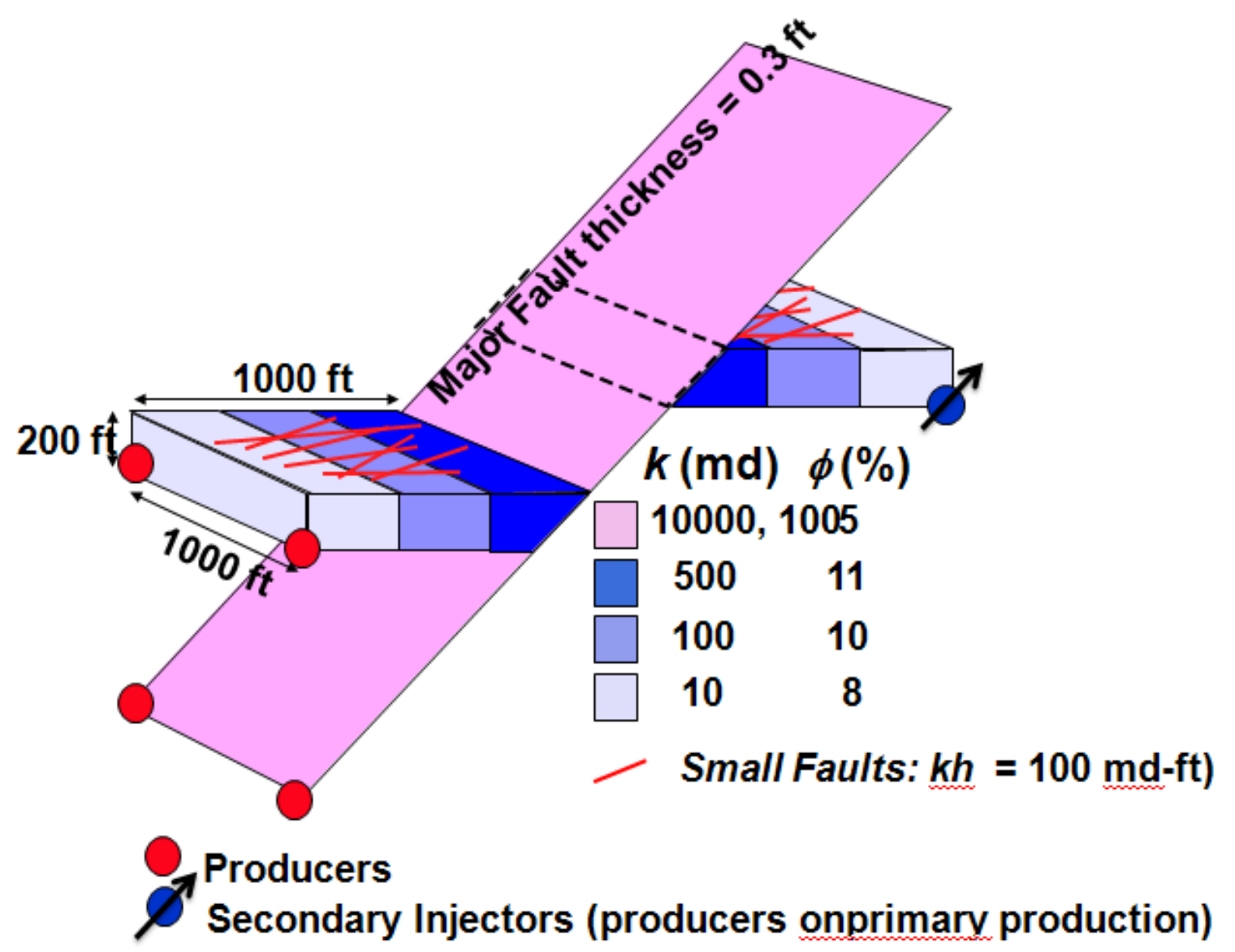

Figure 6.6 Illustration of three-dimensional heterogeneous type II fractured reservoir connected by normal fault 


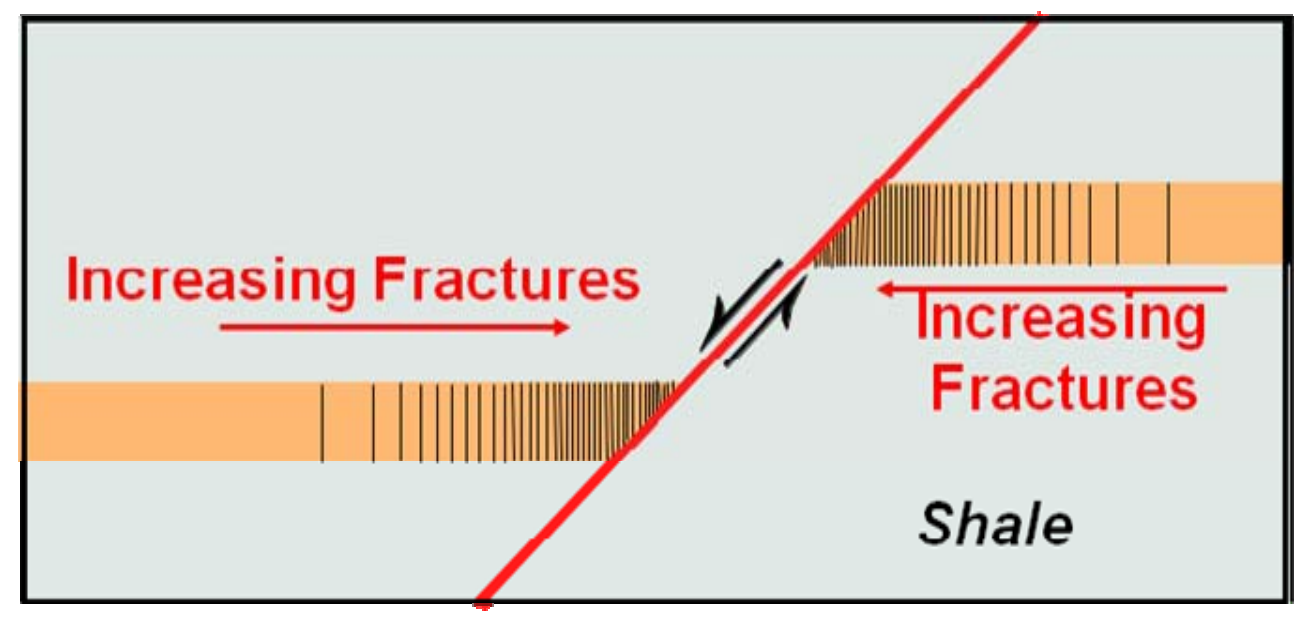

Figure 6.7 Cross-section of type II reservoirs connected by normal dip-slip fault

The dimensions of the two connected reservoirs are the same (1000 by 1000 by $200 \mathrm{ft}$ ). The fractures inside of each reservoir have different orientation and size. The connecting fault is 1000 by 2642 by $0.3 \mathrm{ft}$ with dip angle of $45^{\circ}$.

As described in Table 6.2, two simulations were performed on this conceptual model: normal fault with 10000 md permeability case and $100 \mathrm{md}$ permeability case. Other than the normal fault permeabilities, all of the simulation conditions are exactly the same between these two simulations. The total simulation time was 6000 days with the first 600 days as the primary production time and the secondary production thereafter. During the primary production period, all six wells were operated as the producer with bottom hole pressure (BHP) control at $2200 \mathrm{psi}$. In the primary production stage, the reservoir driving force was combined of the solution gas driving and the gravity driving. As the pressure dropped, more and more solution gas was released from the oil phase to keep the reservoir pressure. Starting from day 601, two wells on the bottom corner at the upper reservoir were turned into the injector and began injecting water. These two converted water injector are operated by BHP control which pressure is 1000 psi higher than the operating conditions of the rest producers. This reservoir production stage was 
called secondary production period. The secondary productions were kept until the end of the simulation. Some results (such as oil recovery percentage, oil production rate, water cut) of these simulations are compared in the rest of this section. 
Table 6.2 Summary of data for modeling type II reservoirs connected by normal fault

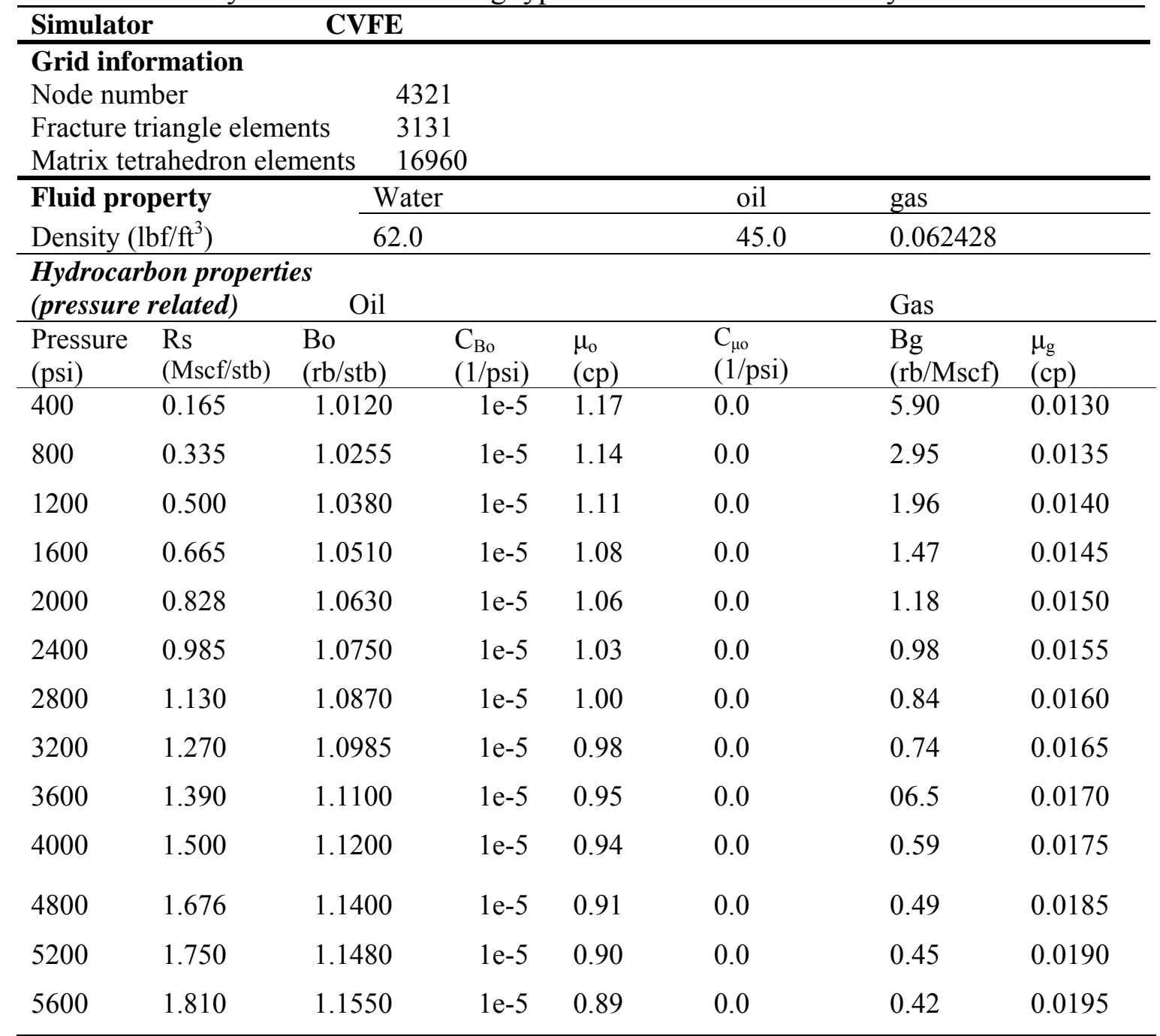

Water properties $^{*}$

Reference pressure ( $p_{\text {ref, }}$ psi)

14.7

Water formation factor $B_{\mathrm{wr}} @ 0.0142$

reference pressure $(\mathrm{rb} / \mathrm{stb})$

$\mathrm{B}_{\mathrm{wr}}$ 's compressibility (1/psi) $\quad 1.0 \mathrm{e}-6$

Water viscosity $\left(\mu_{\mathrm{wr}}\right) @$ reference 0.96

pressure (cp)

$\mu_{\mathrm{wr}}$ 's compressibility $(1 / \mathrm{psi})$

0.0

\begin{tabular}{ll}
\hline Rock property & \\
$\Phi$ (matrix) & 0.11 \\
$\Phi$ (fracture) & 0.0 \\
$\Phi$ (fault) & 0.05 \\
$\mathrm{k}$ (matrix) (md) & $10,100,500$ \\
$\mathrm{k}$ (fracture) (md-ft) & 100 \\
$\mathrm{k}$ (fault) & 10000,100 \\
\hline
\end{tabular}


Table 6.2 Continued

\begin{tabular}{|c|c|c|c|c|}
\hline \multicolumn{5}{|c|}{ Rock-fluid data (Oil-water system) } \\
\hline $\mathrm{S}_{\mathrm{w}}$ & $\mathrm{k}_{\text {row }}$ & $\mathrm{K}_{\mathrm{ro}}$ & $\begin{array}{l}\mathrm{p}_{\text {cow }}(\mathrm{psi}) \\
\text { (matrix) }\end{array}$ & $\begin{array}{l}\mathrm{p}_{\text {cow }}(\mathrm{psi}) \\
\text { (fracture) }\end{array}$ \\
\hline 0.20 & 0.00 & 1.00 & 7.0 & 0.0 \\
\hline 0.30 & 0.07 & 0.40 & 4.0 & 0.0 \\
\hline 0.40 & 0.15 & 0.125 & 3.0 & 0.0 \\
\hline 0.50 & 0.24 & 0.0649 & 2.5 & 0.0 \\
\hline 0.60 & 0.33 & 0.0048 & 2.0 & 0.0 \\
\hline 0.80 & 0.65 & 0.00 & 1.0 & 0.0 \\
\hline 0.90 & 0.83 & 0.00 & 0.5 & 0.0 \\
\hline 1.00 & 1.00 & 0.00 & 0.0 & 0.0 \\
\hline \multicolumn{5}{|c|}{ Gas-water system } \\
\hline $\mathrm{S}_{\mathrm{w}}$ & $\mathrm{k}_{\mathrm{rgw}}$ & $\mathrm{K}_{\mathrm{rW}}$ & $\begin{array}{l}\mathrm{p}_{\mathrm{cgw}}(\mathrm{psi}) \\
\text { (matrix) }\end{array}$ & $\begin{array}{l}\mathrm{p}_{\text {cgw }}(\mathrm{psi}) \\
\text { (fracture) }\end{array}$ \\
\hline 0.00 & 0.00 & 1.00 & 0.0 & 0.0 \\
\hline 0.04 & 0.00 & 0.60 & 0.2 & 0.0 \\
\hline 0.10 & 0.022 & 0.33 & 0.5 & 0.0 \\
\hline 0.20 & 0.10 & 0.10 & 1.0 & 0.0 \\
\hline 0.30 & 0.24 & 0.02 & 1.5 & 0.0 \\
\hline 0.40 & 0.34 & 0.00 & 2.0 & 0.0 \\
\hline 0.50 & 0.42 & 0.00 & 2.5 & 0.0 \\
\hline 0.60 & 0.50 & 0.00 & 3.0 & 0.0 \\
\hline 0.70 & 0.8125 & 0.00 & 3.5 & 0.0 \\
\hline 0.78 & 1.00 & 0.00 & 3.9 & 0.0 \\
\hline \multicolumn{5}{|c|}{ Initial conditions } \\
\hline $\mathrm{P}(\mathrm{psi})$ & & & \\
\hline $\mathrm{S}_{\mathrm{w}}$ & \multicolumn{2}{|c|}{0.22} & & \\
\hline Rs & \multicolumn{2}{|c|}{$\begin{array}{l}0.22 \\
1.27\end{array}$} & & \\
\hline
\end{tabular}

Boundary conditions

Well conditions

Non-flow boundary conditions

\section{(BHP controlled)}

Well 1 (upper reservoir)

Well 2 (upper reservoir)

Well 3 (lower reservoir)

Well 4 (lower reservoir)

Well 5 (fault)

Well 6 (fault)

\begin{tabular}{ll} 
Primary production & Secondary production \\
\hline $2200 \mathrm{psi}$ (producer) & $3200 \mathrm{psi}$ (injector) \\
$2200 \mathrm{psi}$ (producer) & $3200 \mathrm{psi}$ (injector) \\
$2200 \mathrm{psi}$ (producer) & $2200 \mathrm{psi}$ (producer) \\
$2200 \mathrm{psi}$ (producer) & $2200 \mathrm{psi}$ (producer) \\
$2200 \mathrm{psi}$ (producer) & $2200 \mathrm{psi}$ (producer) \\
$2200 \mathrm{psi}$ (producer) & $2200 \mathrm{psi}$ (producer)
\end{tabular}

* Water properties (pressure related) were calculated with the Equations (6-4) and (6-5).

$$
\begin{aligned}
& \mathrm{E}_{\mathrm{Wp}}=\mathrm{B}_{\mathrm{wpp}} \exp \left[-\mathrm{C}_{\mathrm{BW}}\left(\mathrm{P}-\mathrm{P}_{\mathrm{pef}}\right)\right] \\
& H_{\mathrm{Wp}}=H_{\mathrm{Wpp}} \exp \left[-\mathrm{C}_{\mu \mathrm{WF}}\left(\mathrm{P}-\mathrm{P}_{\mathrm{pef}}\right)\right]
\end{aligned}
$$


Oil recovery results are shown in Figure 6.8. As was observed, the recovery rate in the higher fault permeability case was much greater than in the lower fault permeability case. For both studies, oil recovery rates slowed when they entered the secondary recovery stage. This was due to the reduction of production wells (all six wells produced at the primary production and two wells at the upper reservoir were turned into injectors). Quantitatively, at the end of the primary production period, oil recovery in the higher fault permeability case was $13 \%$ compared with $7 \%$ with the lower fault permeability case. This represents an approximately $6 \%$ difference based on total reservoir capacity. At the end of simulation (day 6000), the higher fault permeability case gave $32 \%$ total recovery and the lower fault permeability case offered $14 \%$ total recovery. The difference between the two cases was about $18 \%$ of total oil in place.

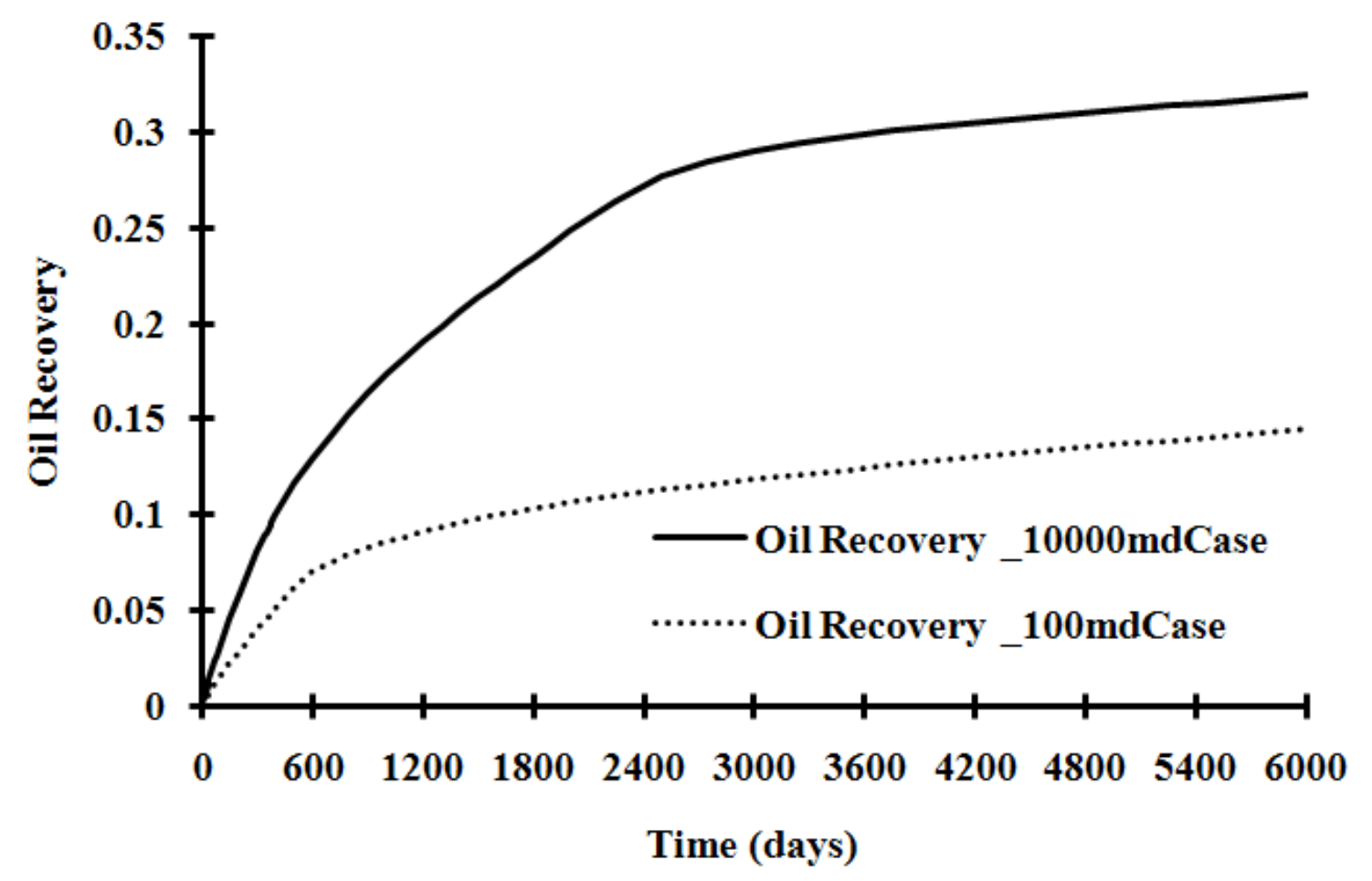

Figure 6.8 Oil recovery comparisons between two case studies (10000 md and $100 \mathrm{md}$ fault permeabilities) 
Meanwhile, the higher fault permeability case offered double the amount of oil recovery than the other case. For the higher fault permeability case the oil recovery curve could be cut into three stages: day 1 to 600 (primary production), day 601 to about 2250, and after day 2250. The slopes of oil recovery fractions declined with time. The first rate reduction has been explained (well number reductions). The second slope turning point (around day 2250) could be explained by the water breakthrough on the two wells located at the bottom of the normal fault. There was no clear oil recovery curve slope change (on the secondary production period) in the lower fault permeability case. After day 2250 , both curves showed close slopes which means they had a similar oil production rate. This can be observed in Figure 6.9. Since behaviors of production wells on the lower reservoir and fault were different in each case study, oil produced from the reservoir and the fault are presented separately.

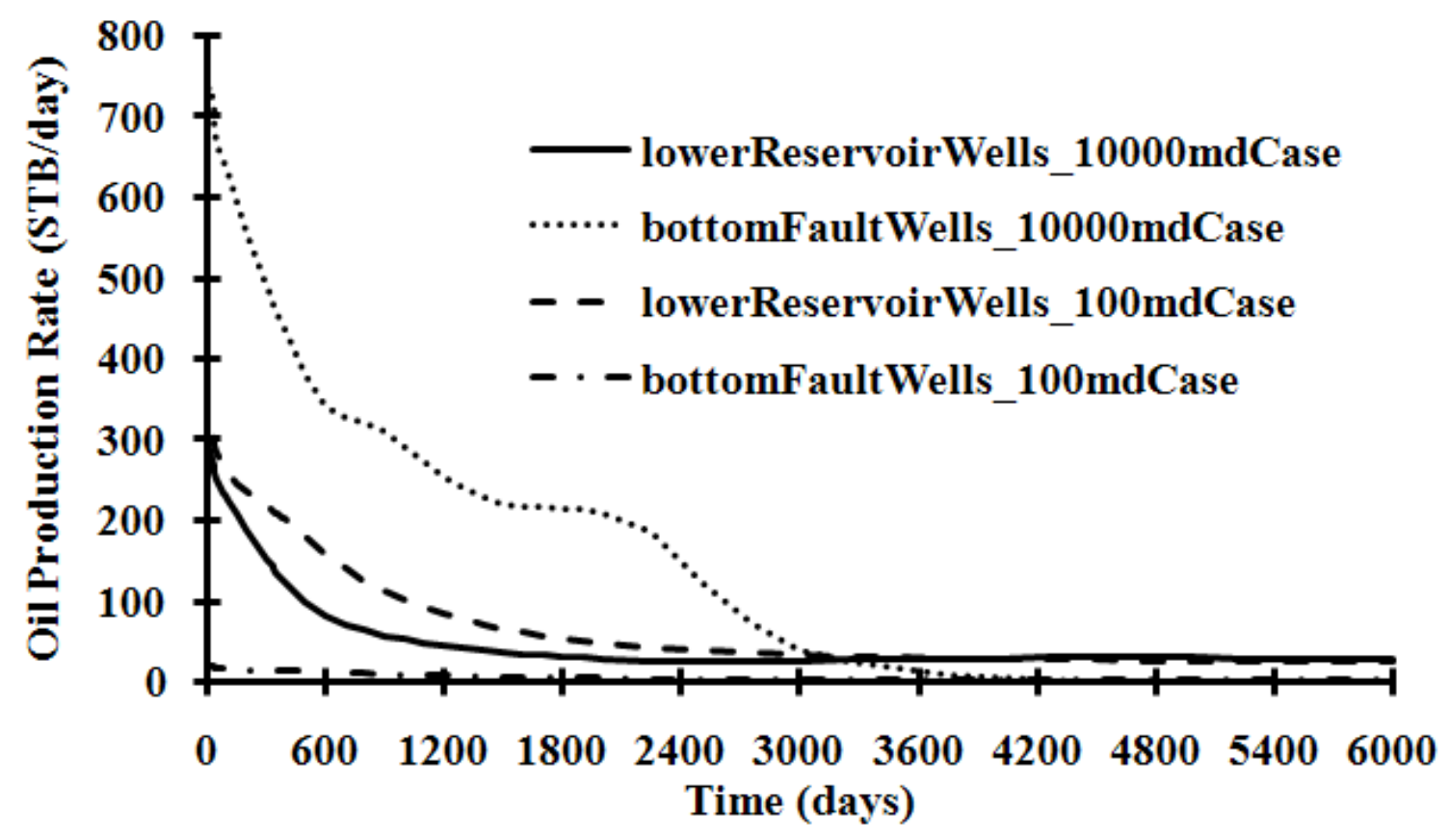

Figure 6.9 Oil production rate comparisons between two case studies $(10000 \mathrm{md}$ and $100 \mathrm{md}$ fault permeabilities) 
The oil production rate and water cut results shown in Equations (6.9 and 6.10) clearly depict the well behaviors with normal fault permeability differences and the well locations. In the first simulation with $10000 \mathrm{md}$ fault permeability, the production wells on the bottom faults contributed most of the oil production until their water cuts reached very high points. This curve could be divided into five stages: from day 1 to 600 , the production rate sharply declined due to the reservoir pressure decrease; from day 601 to day 1400 , the production rate decreased due to reduced producers and water injection; from day 1401 to day 2250 , a stable production stage, water injections pushed oil from upper reservoir to producers on the bottom of the fault; from day 2250 to 4000 , more and more water was produced by these wells due to the breakthrough (at day 3250 , oil produced from fault producers was equal to the oil produced from lower reservoir producers, and at day 4000 , the water cut from fault producers was over $96 \%$ ); from day 4000 to the end of simulation, oil production on the fault producers dropped from $5.35 \mathrm{stb} /$ day to 0.64 stb/day with the highest water cut of $99.7 \%$. However, for the fault producers on the simulation with $100 \mathrm{md}$ fault permeability, the water breakthrough never happened due to the lower fault permeability. The oil production rate on those wells changed only mildly during the whole simulation period. The oil production rate figure suggests an interesting phenomenon in the behavior of lower reservoir producers in these two case studies. The lower reservoir producers with $100 \mathrm{md}$ fault permeability case produced more oil than the higher fault permeability case; this might be due to a combination of higher near-fault matrix permeability effects and the gravity effect. Since the near-fault matrix has $500 \mathrm{md}$ permeability and the fault has only 100 md, part of the oil flow from the top reservoir or upper fault might have gone into the lower matrix instead of flowing directly down to the bottom fault producers. Furthermore, the water cut figure shows that the lower reservoir producers in the $10000 \mathrm{md}$ fault permeability case had 
a certain amount of water produced during the simulation but not in the other case with $100 \mathrm{md}$ fault permeability. Some snapshots of oil saturations might help to understand these physical behaviors.

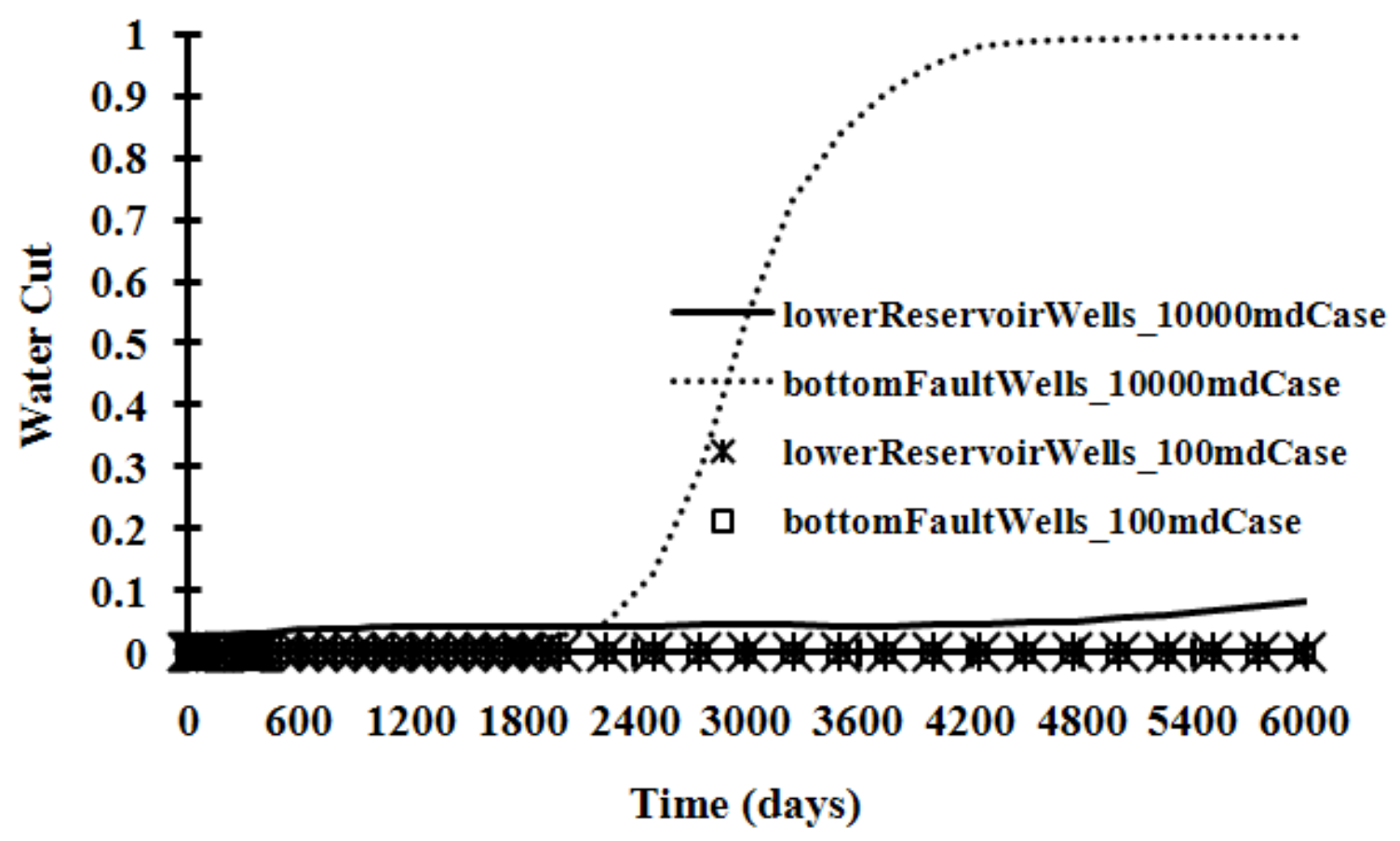

Figure 6.10 Water cut comparisons between two case studies (10000 md and $100 \mathrm{md}$ fault permeabilities)

The snapshots of oil saturations below present the fault permeability impact on oil production (Figure 6.11 and Figure 6.12). Two pictures in each row represent the same case on the same time oil saturations but from different viewing angles. For example, on the first row of the 10000 md fault permeability case, two pictures represent the same oil saturation distributions at day 600 (just after the primary productions). The picture on the left gives the west-east view, which is good for watching the front side of the normal fault and the top side of the lower reservoir; the picture on the right was taken from another angle and obtained a clear view point of the rear side of the normal fault and the lower side of the upper reservoir. Comparing the two case studies at 
the end of primary production (day 600), the $10000 \mathrm{md}$ case clearly had more oil drained from both reservoirs and the upper fault to the bottom of the normal fault then the other case. On the pictures of the simulation end, water clearly shows up on the bottom of both reservoirs and the fault in first case with $10000 \mathrm{md}$ fault permeability. For the $100 \mathrm{md}$ fault permeability case, the injecting water front had not yet even reached the normal fault. At day 6000, the oil saturation profile on the lower reservoir in the $10000 \mathrm{md}$ case showed a strap distributed from bottom producers to the top of lower reservoir and the fault. This effect may have been generated for two reasons: 1) the upper oil saturation vacancy (above the lower matrix producers) was generated by gravity, and 2) the lower oil saturation vacancy was from the water injection from upper reservoir transferred by the normal fault and invading from the fault side to the bottom of the lower reservoir.

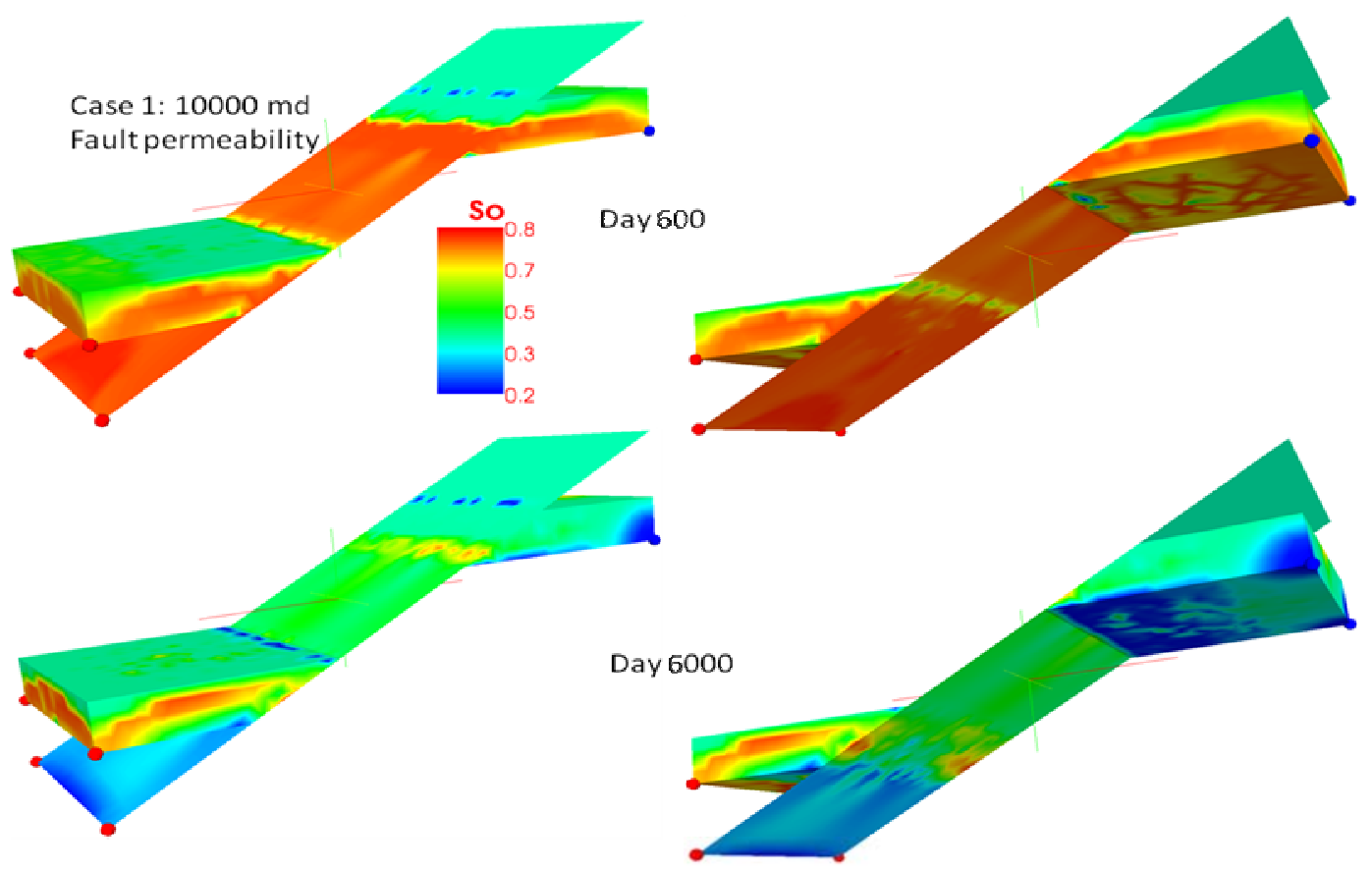

Figure 6.11 Snapshots of oil saturations in $10000 \mathrm{md}$ fault permeability case with day 600 (upper) and 6000 (lower) 


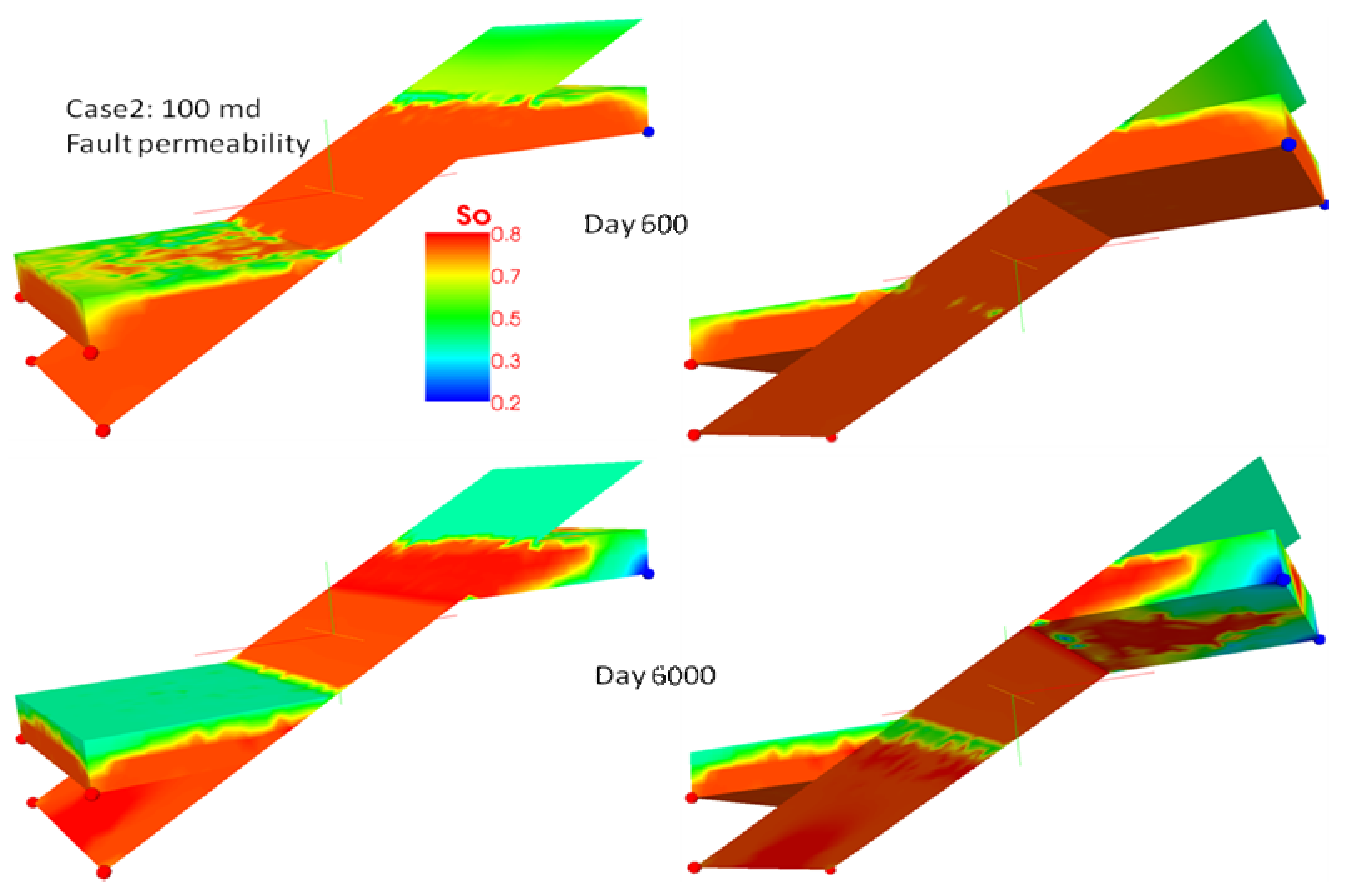

Figure 6.12 Snapshots of oil saturations in 100 md fault permeability case with day 600 (upper) and 6000 (lower)

This study showed the great capability of the CVFE multiphase reservoir simulator in handling complex type II fractured reservoirs. The reservoir's observed behavior agreed well with the common sense expectations of the designed reservoir. Surely there was still room to play around with this conceptual model for different recovery plans, but it was outside of the scope of this study. The fractures in the reservoir certainly formed some fluid flow paths and geometric traps for the hydrocarbons. The normal fault played a key role in the process of oil productions for this kind of hydrocarbon recovery. Practically, if the normal fault had been used in the production, it would have essentially impacted the oil recovery, and brought many uncertainties. The fault has to be carefully characterized in the flow model and the simulation tool has to be carefully selected as well. 


\section{Outcrop to Simulation: “Teasdale Fault” Line Drive Scenario: Heterogeneous Matrix with Small Fracture}

This was another multiphase type II reservoir study. In this section, the Teasdale fault, as shown in Figure 6.13, a major left-lateral strike-slip fault zone in southern Utah was chosen to model another important type II fractured reservoir. Distinguished from the dip-slip fault in the last application, the strike-slip fault is usually nearly vertical and the foot wall moves either left or right or laterally with very little vertical motion.

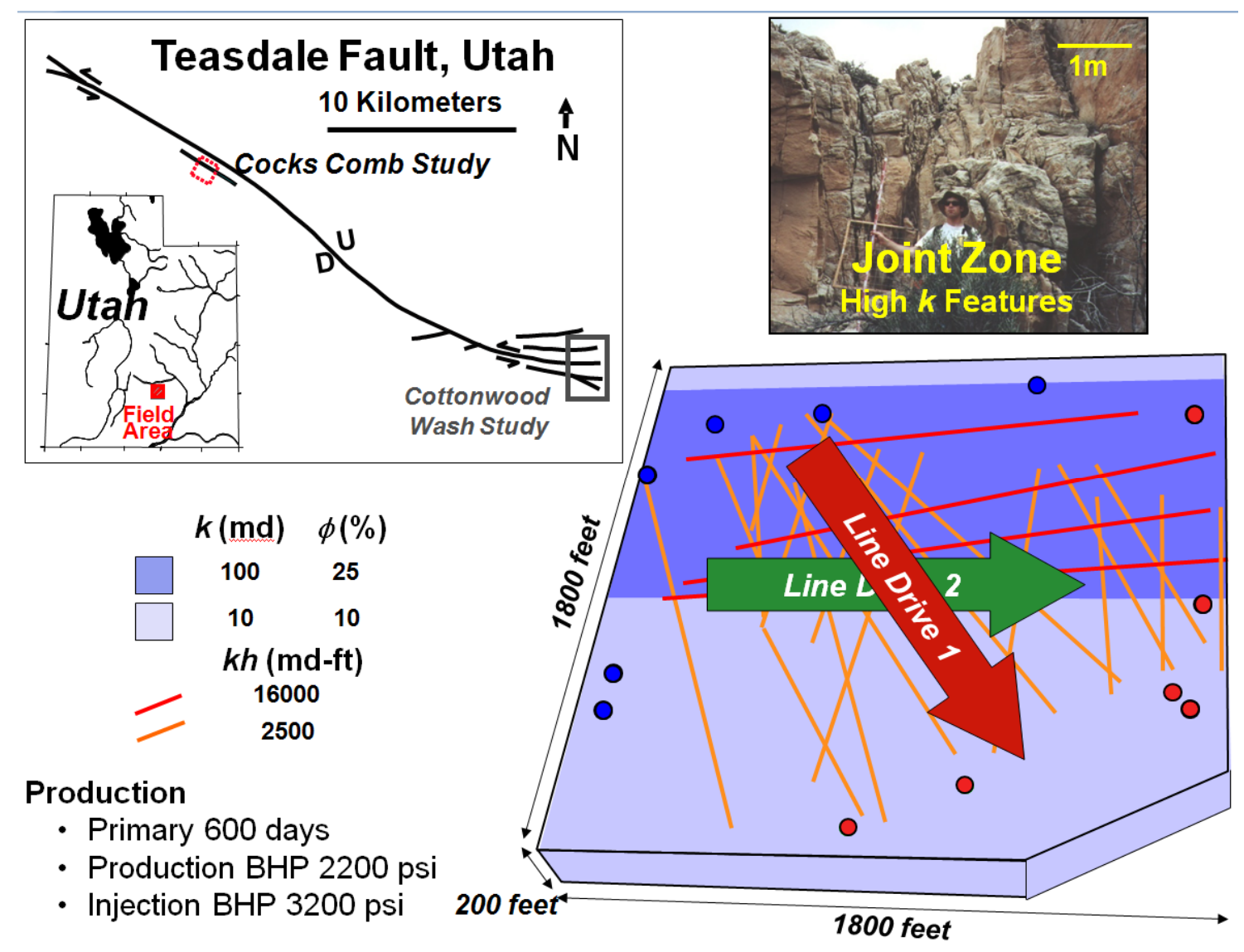

Figure 6.13 Illustration of modeled area, outcrop of joint zone with high permeability features and heterogeneous type II flow model

Outcrop analogs found along the 40-km Teasdale Fault (cutting Aeolian Navajo Sandstone) helped to construct the reservoir model for the multiphase flow purpose. The reservoir model 
was built with the dimension of 1800 by 1800 by $200 \mathrm{ft}$. There were two sets of faults inside the simulation domain: relative low permeability (2500 md-ft conductivity) set in the direction of NE-SW and high permeability (16000 md-ft conductivity) set in W-E direction. The matrix had two different zones: a high permeability matrix zone (with 100 md permeability and 25\% porosity) and a low permeability matrix zone (with 10 md permeability and $10 \%$ porosity). All high permeability set faults (four in total) were located in the high permeability matrix zone. The low permeability set faults crossed both matrix zones. All of the faults were DFN modeled with two-dimensional planes and discretized as finite-element triangles. The reservoir matrix was discretized as finite-element tetrahedrons by conforming to fault triangles. Since most of the fluid properties (density, viscosity, PVT characteristics) in this application were the same as those of the dip-slip fault model (refer to Table 6.2), in this section, only the differences of the critical reservoir data are summarized in Table 6.3 .

As shown in the model illustration and critical data summary, two line drive studies were designed based on two distinguished fault sets. Both studies were three-phase based and hydrocarbon was recovered in two stages-primary pressure recovery and secondary water flooding recovery. There were six wells in each simulation. In the primary recovery stage, all of six wells were producers with 2200 psi BHP control. In the secondary production, three wells on either the north or the west side were turned into injectors with 3200 psi injecting pressure control; the other three wells had the same operating conditions as before. This study was designed to observe the physical impact of the strike-slip fault on the hydrocarbon recoveries.

Figure 6.14 shows that there was no significant difference in the primary production stage. The line drive 2 case study presented a little bit more oil recovery than the line drive 1 case study 
$(7.8 \%: 7.1 \%)$. However, the line drive 2 simulation showed stronger oil recovery fractions than the line drive 1 simulation after the water injection.

Table 6.3 Summary of critical data for modeling Teasdale Fault type II reservoir

\begin{tabular}{|c|c|c|}
\hline \multicolumn{3}{|c|}{ Simulator $\quad$ CVFE } \\
\hline \multicolumn{3}{|l|}{ Grid information } \\
\hline Node number & 5765 & \\
\hline Fracture triangle elements & 3356 & \\
\hline Matrix tetrahedron elements & 27994 & \\
\hline \multicolumn{3}{|l|}{ Rock property } \\
\hline$\Phi$ (matrix) & $0.25,0.10$ & \\
\hline$\Phi$ (fault) & 0.0 & \\
\hline k (matrix) (md) & 10,100 & \\
\hline $\mathrm{kh}$ (fault) (md-ft) & 2500,16000 & \\
\hline \multicolumn{3}{|l|}{ Initial conditions } \\
\hline$P(p s i)$ & 3200 & \\
\hline $\mathrm{S}_{\mathrm{w}}$ & 0.22 & \\
\hline Rs & 1.27 & \\
\hline Boundary conditions & Non-flow boundary conditions & \\
\hline \multicolumn{3}{|l|}{$\begin{array}{l}\text { Well conditions } \\
\text { (BHP controlled) }\end{array}$} \\
\hline Line drive study 1 & $\begin{array}{l}\text { Primary production } \\
(\text { day } 0 \sim 600)\end{array}$ & $\begin{array}{l}\text { Secondary production } \\
(\text { day } 601 \sim 6000)\end{array}$ \\
\hline Well 1 (N-left) & 2200 psi (producer) & 3200 psi (injector) \\
\hline Well 2 (N-middle) & 2200 psi (producer) & 3200 psi (injector) \\
\hline Well 3 (N-right) & 2200 psi (producer) & 3200 psi (injector) \\
\hline Well 4 (SW-left) & 2200 psi (producer) & 2200 psi (producer) \\
\hline Well 5 (SW-middle) & 2200 psi (producer) & 2200 psi (producer) \\
\hline Well 6 (SW-right) & 2200 psi (producer) & 2200 (producer) \\
\hline Line drive study 2 & $\begin{array}{l}\text { Primary production } \\
(\text { day } 0 \sim 600)\end{array}$ & $\begin{array}{l}\text { Secondary production } \\
\text { (day } 601 \sim 6000)\end{array}$ \\
\hline Well 1 (W-upper) & 2200 psi (producer) & 3200 psi (injector) \\
\hline Well 2 (W-middle) & 2200 psi (producer) & 3200 psi (injector) \\
\hline Well 3 (W-lower) & 2200 psi (producer) & 3200 psi (injector) \\
\hline Well 4 (E-upper) & 2200 psi (producer) & 2200 psi (producer) \\
\hline Well 5 (E-middle) & 2200 psi (producer) & 2200 psi (producer) \\
\hline Well 6 (E-lower) & (producer) & 2200 psi (producer) \\
\hline
\end{tabular}




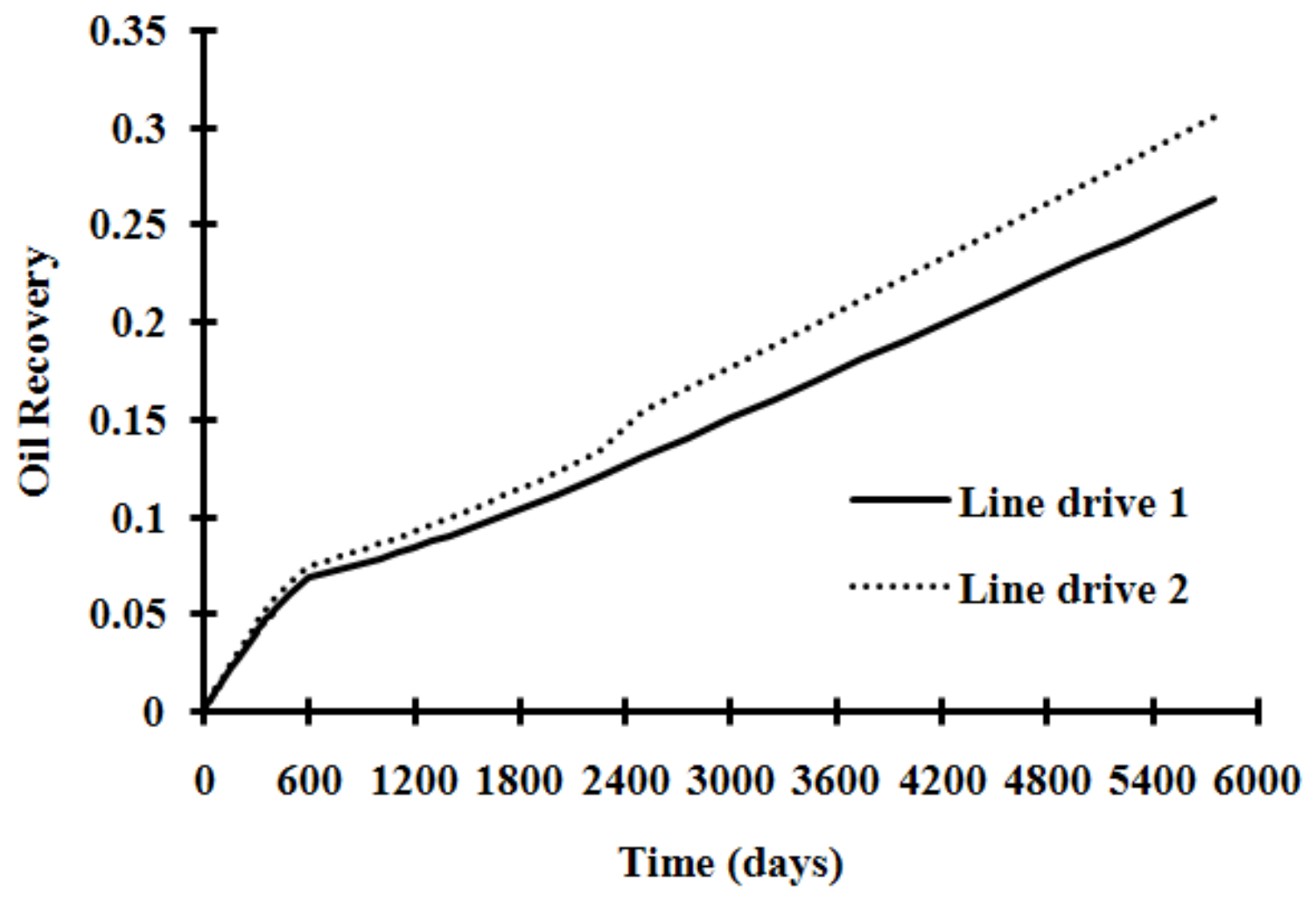

Figure 6.14 Comparisons of oil recoveries between line drive 1 and line drive 2

At the end of simulation, the line drive 1 simulation resulted in $26 \%$ total recovery and the line drive 2 resulted in a $30 \%$ total oil recovery fraction. There was $4 \%$ difference between the two case studies based on original oil in place. These results were consistent with the designed Teasdale type II model. The line drive 2 simulation was designed to flush the reservoir from $\mathrm{W}-$ E by that high permeability fault set (four faults in total). The line drive 1 simulation was designed to use a relatively low permeability fault set (16 faults in total) flush reservoir in $\mathrm{N}-$ SW direction. Some oil saturation distribution snapshots are listed later. Two dates were selected for both simulations: day 600 at the end of primary production and day 6000 at the end of simulation. 
Figure 6.15 shows the faults' effects on the primary production stage. Significantly lower residual oil saturation could be seen on the bottom of the high permeability fault set from the bottom view of line drive 2 simulation (right bottom picture). Since there were more faults in the lower permeability fault set than in the high permeability fault set (17:4), line drive 1 shows more even residual oil distributions than line drive 2 at the end of primary production (pictures in left column). There were more oil concentrations in the upper middle region of the line drive 2 study than there were in the other case. This region had high permeability in both matrix and faults. Since all of the wells in line drive 2 were distributed at either the east of west side of the domain, lower operating pressure $(2200 \mathrm{psi})$ made hydrocarbons migrate from other high pressure regions. Faults had a very important role in collecting oils from nearby places through their "highway" network to transport them to the lower pressure gradient region. It was observed that even though the oil recovery figure showed similar recovery fractions at the end of primary production, the residual oil saturation was distributed very differently for the two recovery scenarios. Practically, this observation could aid in understanding production in a highly heterogeneous type II fractured reservoir in three ways:

- Could help to set up well networks based on fault properties such as orientation and permeability.

- Could help to trace hydrocarbon migrations in the primary production by simulation.

- Could help to make decisions for setting up the injection wells in the secondary recovery stage. 

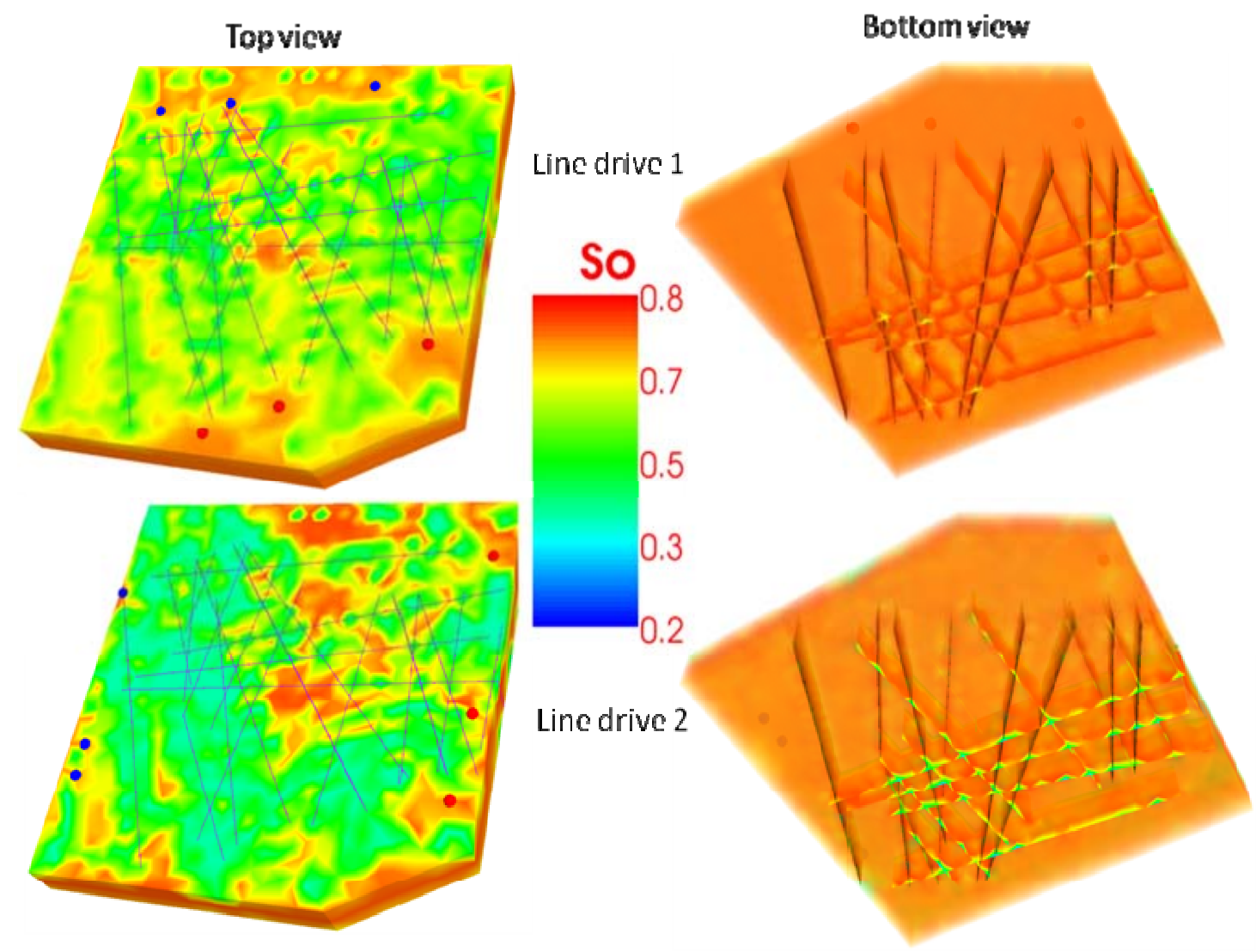

Figure 6.15 Oil saturation comparisons between line drive 1 and line drive 2 at the end of primary production (day 600)

The oil saturation distribution snapshots at the end of simulation (day 6000) are shown in Figure 6.16. Figure 6.17 shows that there was no significant difference in the secondary production stage.

Water flooding patterns are visible for both line drive studies at the end of simulation in Figure 6.16. Especially from the bottom view of both cases, the higher permeability fault set trapped significant water (blue) than the other fault set. Even though two injectors and two producers were set up on the low permeability matrix zone in the case study of line drive 2, the water front in the higher matrix permeability zone was still far beyond that of the low matrix zone from the bottom view pictures. It was also possible to observe from both case studies that the oil 
saturation at the bottom was much lower than at the top. This was especially true near the injection wells. Physically, this was due to the gravity effect: water is much denser than oil (62:45). As shown in the domain description at the beginning of this application, the thickness of this reservoir model was $200 \mathrm{ft}$ and the gravity effects happened very soon after flooding water was injected. Compared to the results at the end of primary production, the top view of the oil saturation snapshot at the end of simulation showed that higher oil concentration was pushed from injection wells to the production wells.
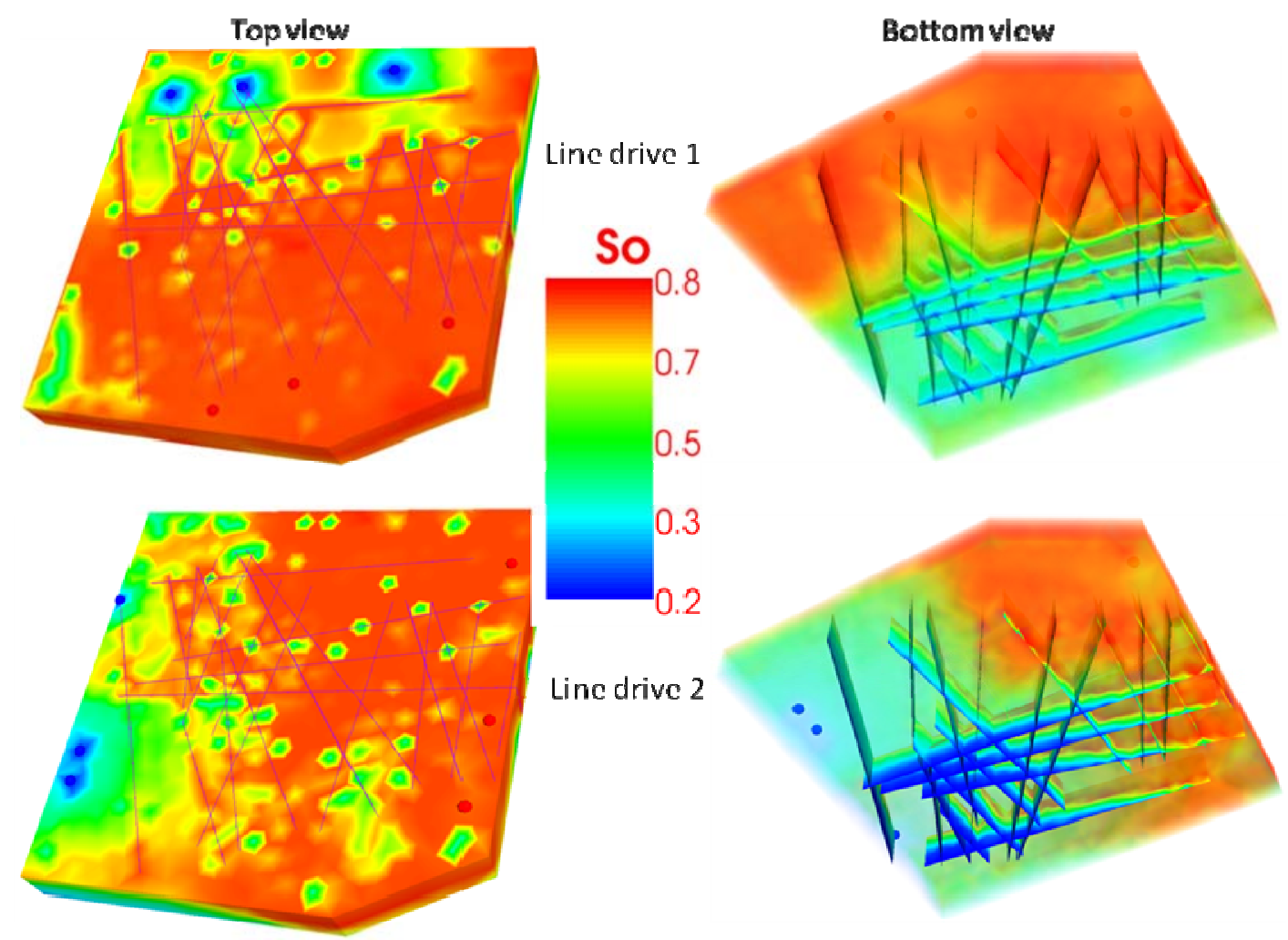

Figure 6.16 Oil saturation comparisons between line drive 1 and line drive 2 at the end of simulation (day 6000) 


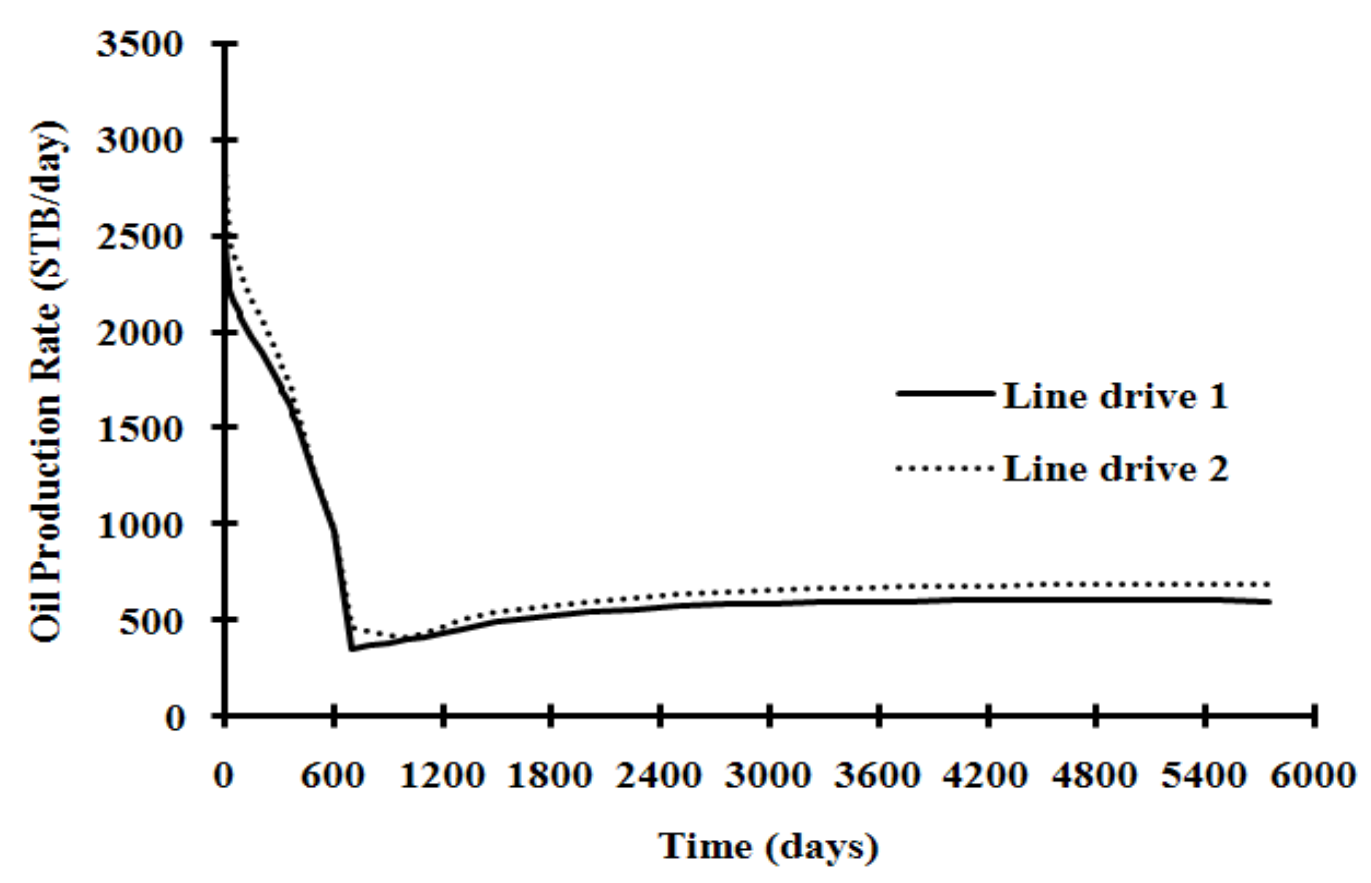

Figure 6.17 Oil production rate comparisons between two line drive case studies

Figure 6.18 shows that line drive 2 case study had a higher oil production rate than the other case all through the simulation. At the primary stage, the difference was about $0.6 \%$ to $11.2 \%$. There was a sharp increase for the rate difference right after water injection (up to $34.2 \%$ ). Then it quickly declined to $4.4 \%$. This observation could be explained by one injector on line drive 1 being located on the corner of a fault. The injecting pressure could be quickly transferred through the fault network to push more oil out of the producers. The location of this specific well could also be used to explain the production rate difference between the two case studies. After the big jump and decline at the beginning of secondary recovery, the oil production rate difference in the line drive 2 case study had a couple more relatively sharp climbs which were also counted as the fault network effect and eventually showed smooth and slow increase through the simulations. The rate difference between the two case studies was about $10 \% \sim 15 \%$. This could give the conclusion that in the type II fractured reservoir the water flooding pattern should be carefully selected based on the fracture/fault characteristics. 


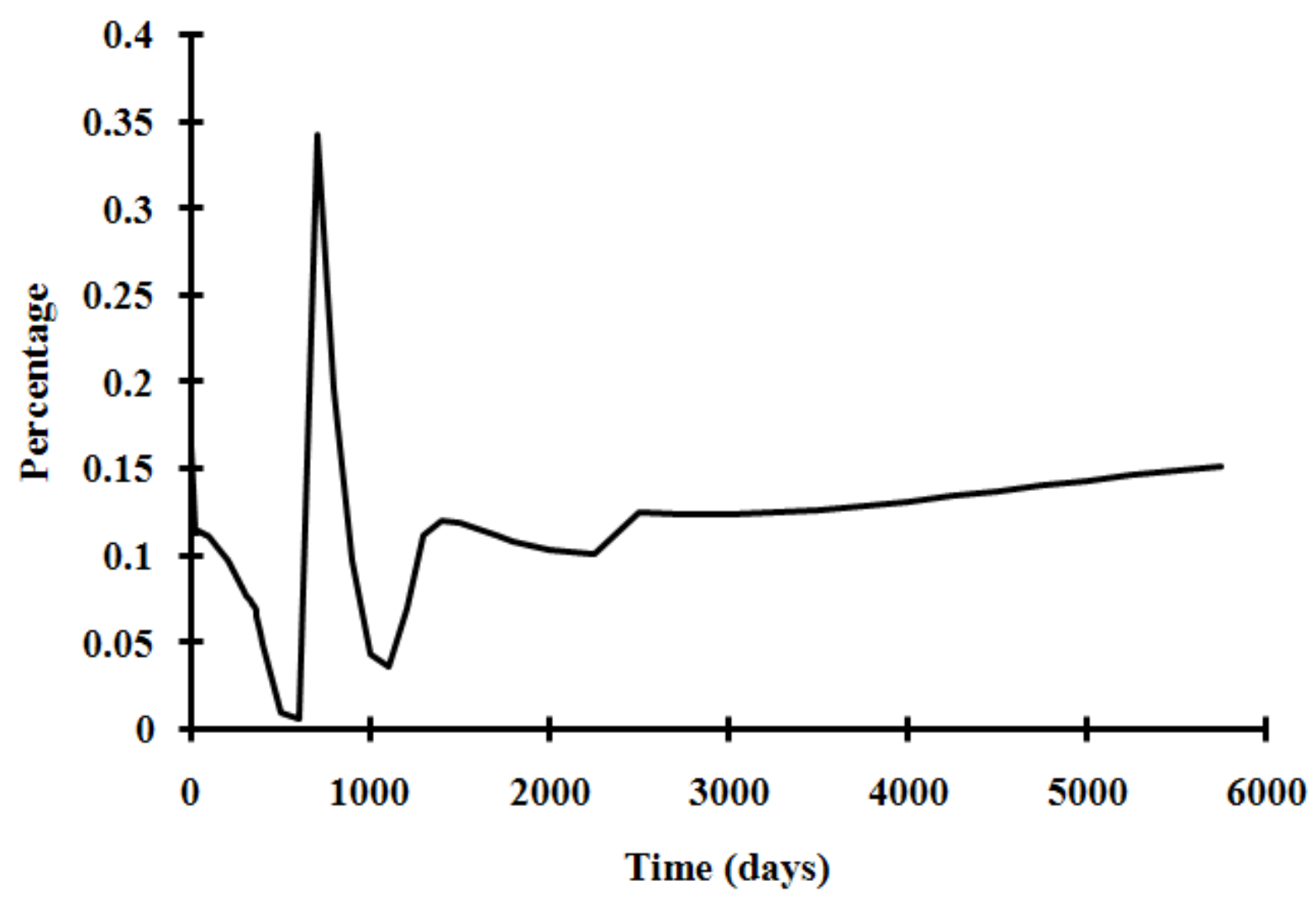

Figure 6.18 (Line drive 2 oil rate - Line drive 1 oil rate)/ Line drive 1 oil rate

\section{"Preferred Pathway" on Type II Reservoir}

Some "preferred pathway" case studies have been performed on the type I reservoirs and described previously (Chapter 3). For type II fractured reservoir, if injection and production wells happened to be located on the connected high permeability fracture/fault network, the short-circuit flow path will be formed and will lower oil recovery efficiency dramatically. In this application, the Teasdale Fault domain was being simplified to study this type of physical phenomena. Instead of a highly heterogeneous type II reservoir model used in last application, the matrix in this study was assigned homogeneous porosity $(12.7 \%)$ and permeability $(0.5 \mathrm{md})$. There are three simulations in this study: 
1) Base case (with $500 \mathrm{md}-\mathrm{ft}$ fault transmissivity);

2) Preferred pathway case $(500,000 \mathrm{md}-\mathrm{ft}$ on preferred pathway and $500 \mathrm{md}-\mathrm{ft}$ on rest faults);

3) Preferred pathway with cut-off zones $(500,000 \mathrm{md}-\mathrm{ft}$ on preferred pathway, $0.5 \mathrm{md}-\mathrm{ft}$ on cut-off pathway zones and $500 \mathrm{md}-\mathrm{ft}$ on the rest of faults).

This case study was confined as two phases (oil and water). There were two wells in total and they were located at the two ends of preferred pathway (as shown in domain description below). The critical data are summarized in Table 6.4.

The circled faults (four in total) in the above domain (Figure 6.19) were assigned with high permeability (500000 md-ft) in the simulation of preferred pathway. The fault circled in black at the middle was treated as the "cut-off" fault zone which was assigned to $0.5 \mathrm{md}-\mathrm{ft}$ permeability in the simulation of preferred pathway with cut-off zone studies. The injection well was marked as a blue dot on the end of the southwest fault and the production well was marked as a red dot on the corner of the northeast fault as shown in the domain description. In this application, water was injected from day 1 and total simulation time was 10,000 days. The oil saturation snapshots of different times are shown in Figure 6.20.

Figure 6.20 shows that the water flooding patterns are totally different due to the existence of high permeability fault short-circuit in the type II fractured reservoirs. The first row of pictures in the figure above represents the simulation results of day 100; the base case study showed much shorter water injection trajectories than the other two cases due to the difference of fault permeabilities. When comparing the preferred pathway case in the middle column with the preferred pathway with cut-off zone case in the last column, it is clear that the injecting water 
was flowing through the highest permeability fault to the producer. The following observations can be made from the three case studies over the entire simulation:

1) The base case showed a relatively even water saturation front from the injector to the producer. Injecting water could push oil out of the matrix to nearby faults and then use the fault as its transportation tool sending reservoir fluid into the producer.

2) The preferred pathway case showed that only oil near high permeability faults would flood out. Most oil in the relatively low permeability faults did not get the chance to flood out.

3) The preferred pathway with cut-off zone case study showed that most of oil between two high permeability faults was flooded out at the end of simulation. 
Table 6.4 Summary of critical data for modeling type II reservoirs connected by normal fault

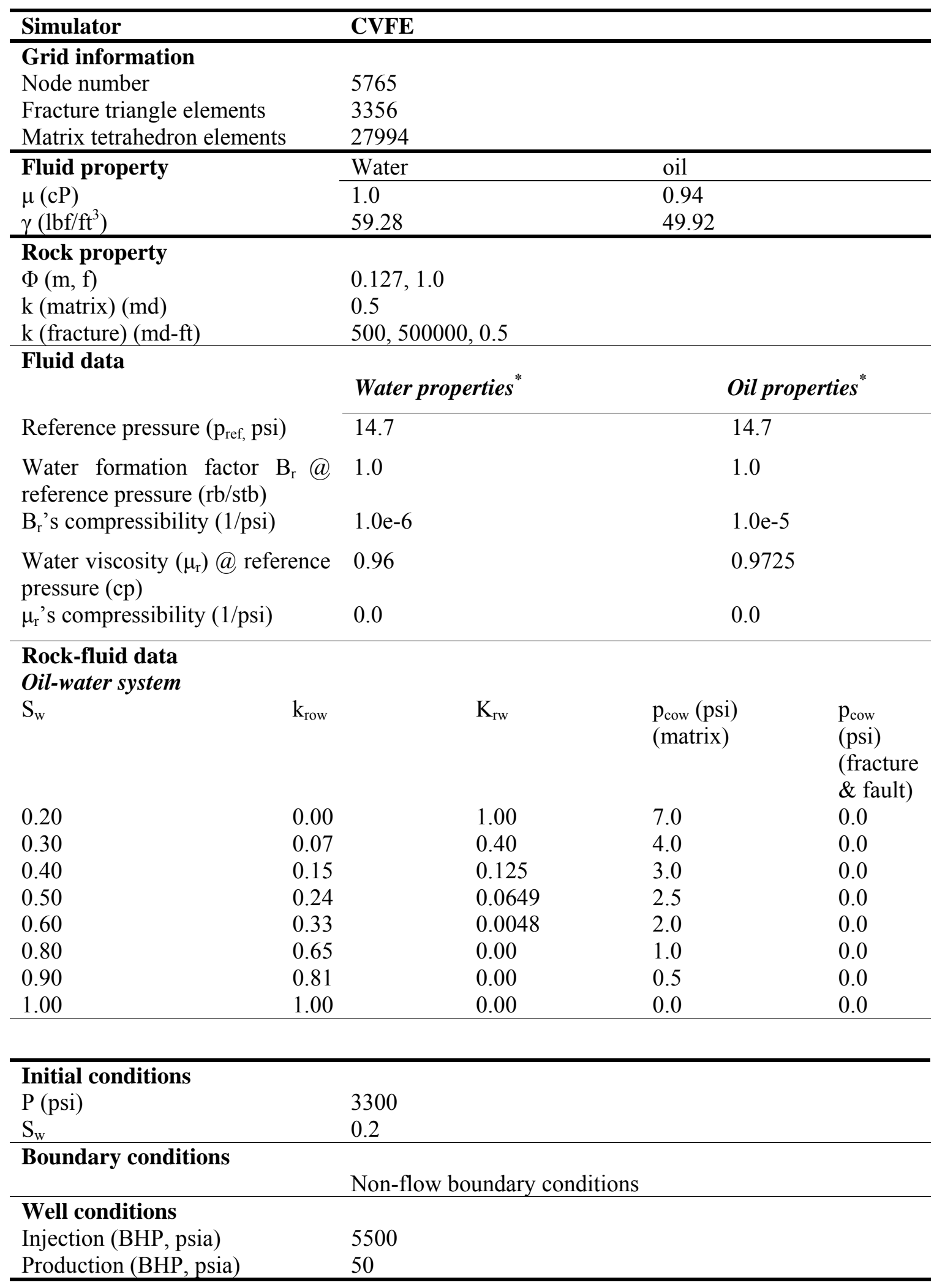




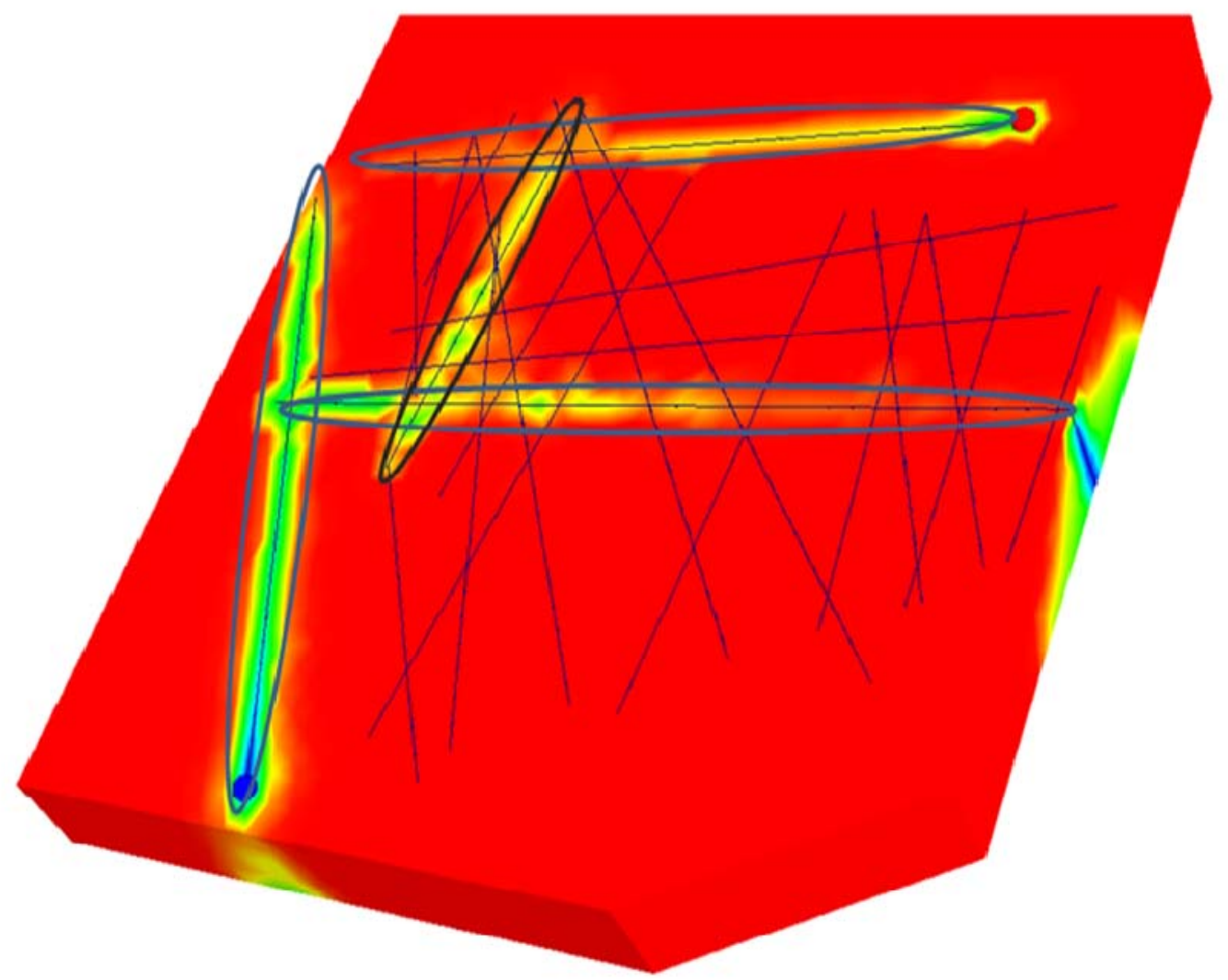

Figure 6.19 Illustration of preferred pathway characterization in type II fractured reservoir and preferred pathway with cut-off zone (circled). 

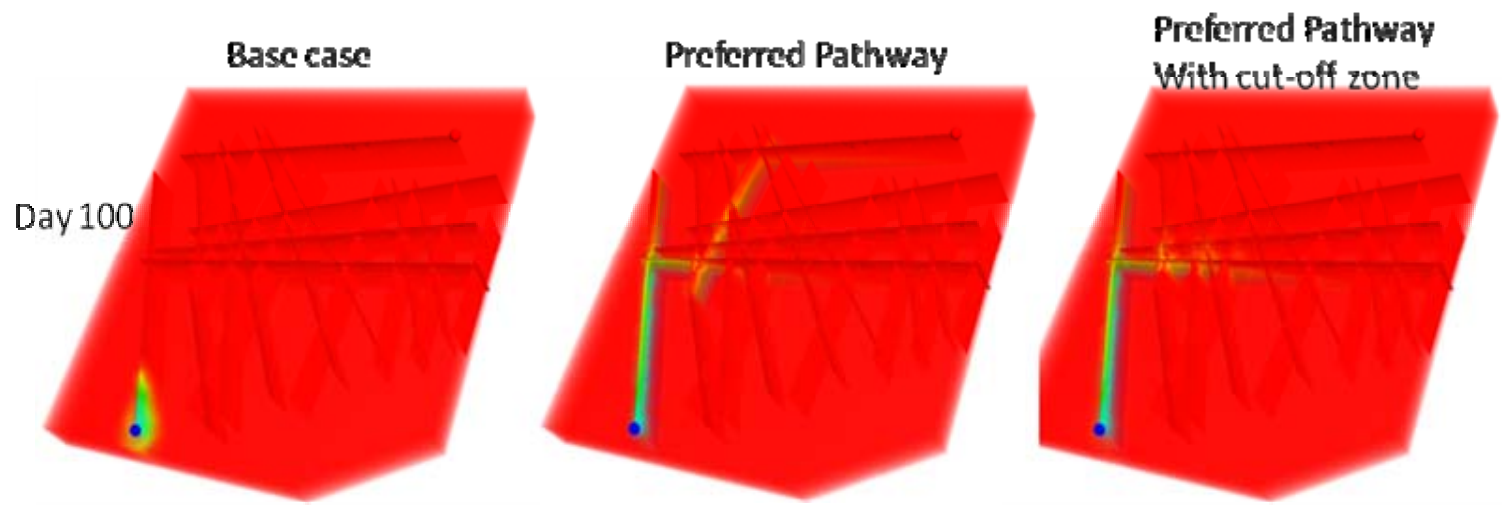

1)
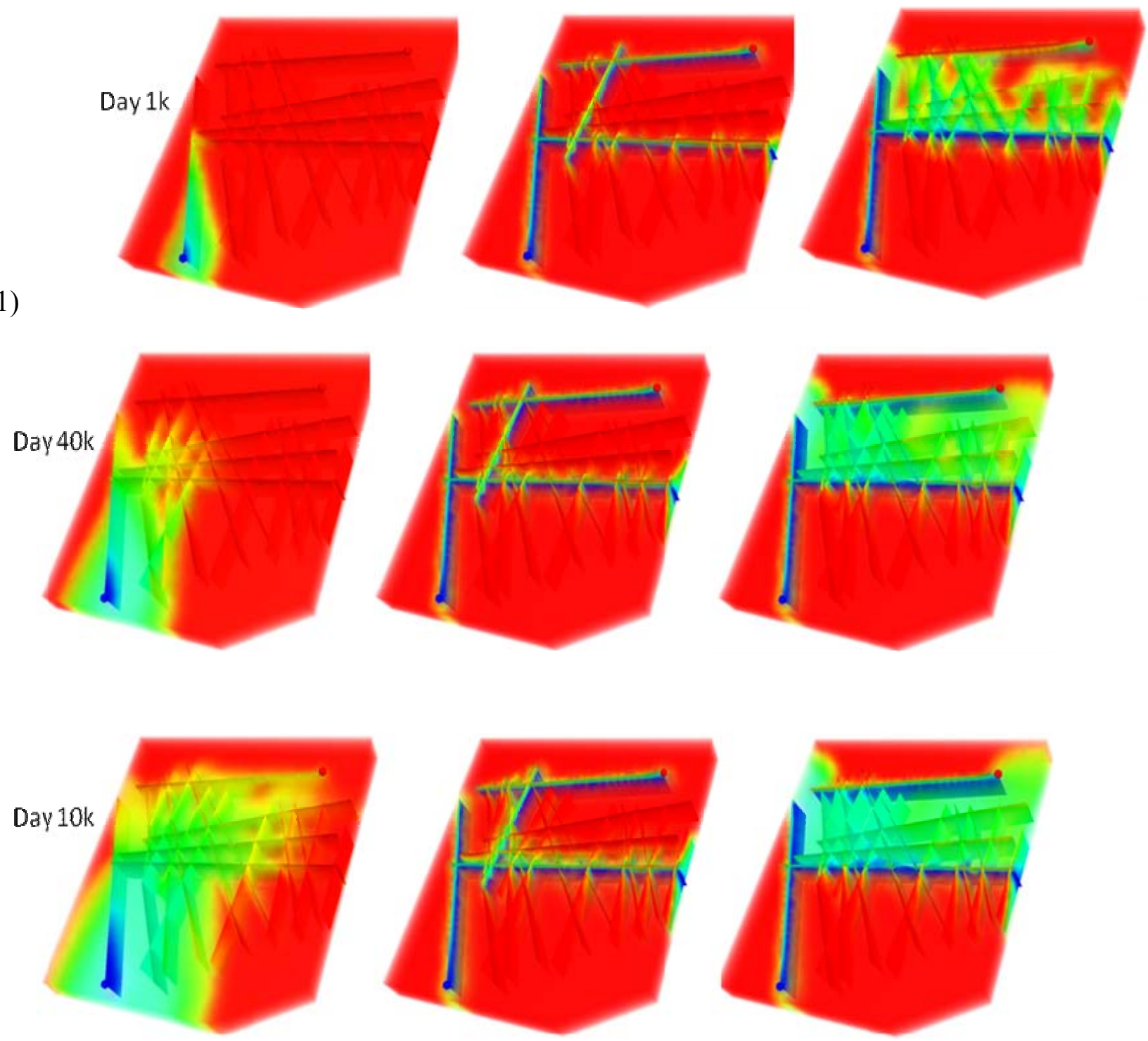

Figure 6.20 Oil saturation distribution comparisons among different fault permeabilities on different time scales 
Figure 6.21 shows that at the beginning of the simulation, for a very short period both preferred pathway and cut-off zone cases had identical oil recovery rate, which was higher than the base case. This was due to the existence of a higher permeability fracture/fault near the injector. After a while, the trend of oil recoveries in the recovery figure displayed the recovery rate with the order from high to low: the cut-off zone case, the base case and the preferred pathway case. This was very encouraging in regard to the idea of treating higher permeability fractures/faults with special techniques such as gels in order to reduce or block the water flow. Such treatment can dramatically increase oil production.

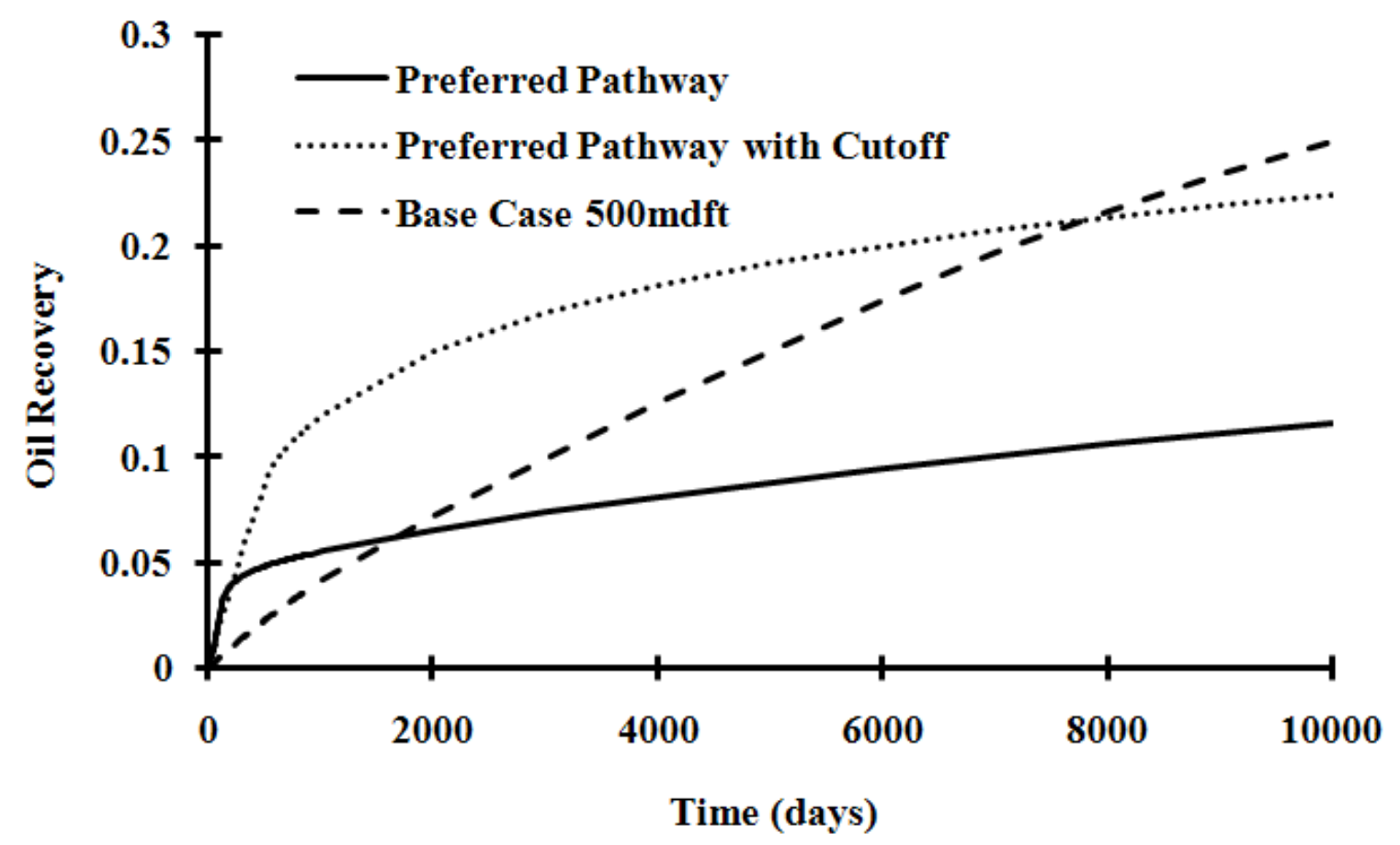

Figure 6.21 Oil recovery fraction comparisons for preferred pathway type II fractured reservoir studies

Figure 6.22 showed that the existence of ultrahigh permeability short-circuits could be either an enemy or friend during oil recovery. Geologically, those ultrahigh permeability faults exist externally. However, the negative effects from high permeability fault existence could also be 
converted into a preponderant element if the correct production strategies were designed or the right treatments were applied. In this application, the base case showed more consistent production behavior than the other two cases. The ratios of initial and end-day oil production rates were 5.12 (base case), 58.47 (preferred pathway case) and 54.48 (cut-off zone case). The oil recoveries at the end of simulation (day 10000) were $25 \%$ (base case), $11 \%$ (preferred pathway case) and $22 \%$ (cut-off zone case).

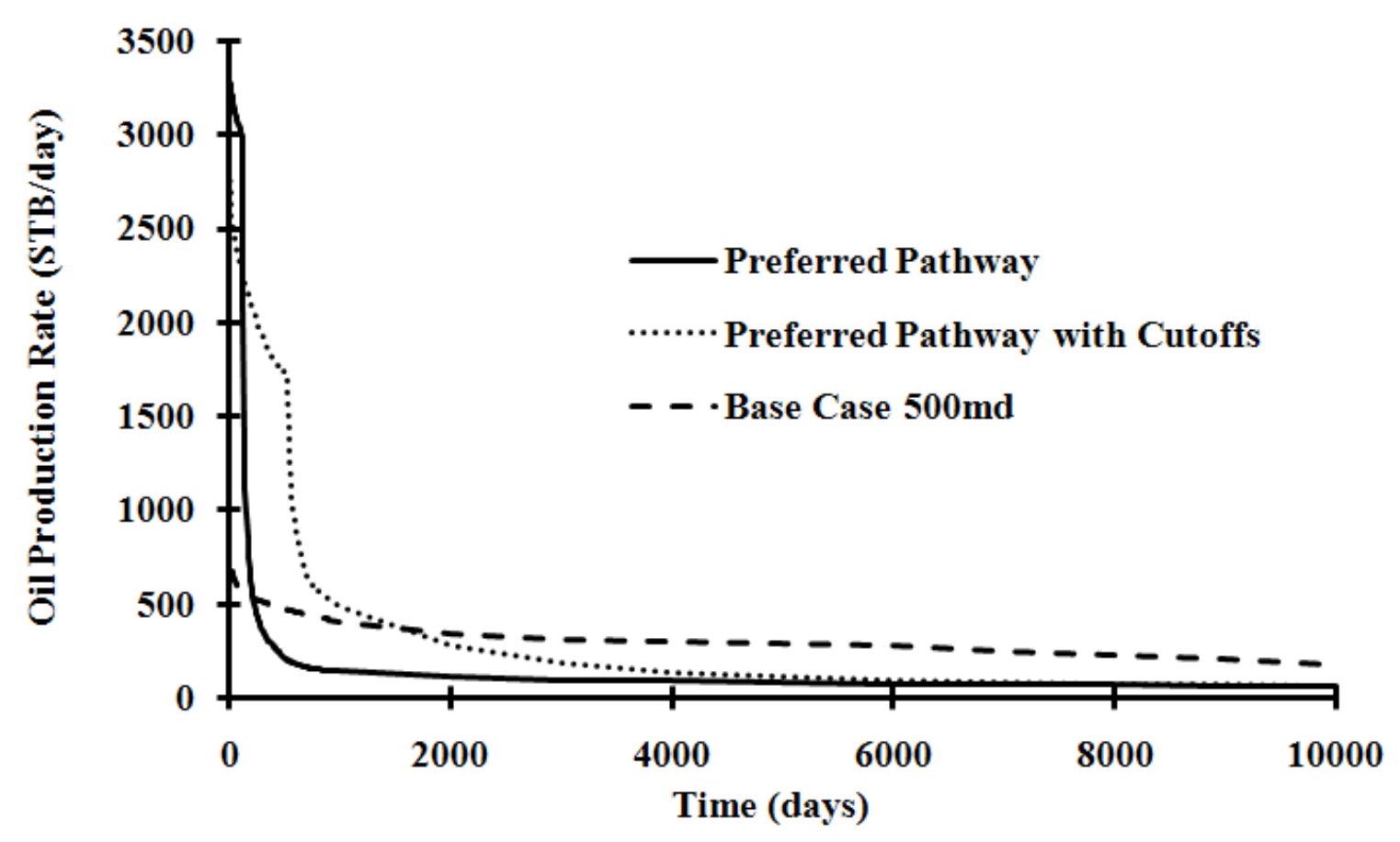

Figure 6.22 Oil production rate comparisons for preferred pathway type II fractured reservoir studies

The plot of oil recovery with injection pore volume (PV) in Figure 6.23 explains the costeffectiveness ratio for production oil from these type II fractured reservoirs. To achieve $10 \%$ oil recoveries, the base case required $0.07 \mathrm{PV}$ injections; the preferred pathway case needs $0.84 \mathrm{PV}$ water injections; and the cut-off zone case needs $0.10 \mathrm{PV}$ injections. However, to get the same oil recovery, the time spent on each case is 3000,7000 and 610 days. Obviously the cut-off zone model offered the best production plan to reach this goal by combining these two analyses. Being 
distinguished from the type I basement reservoir, type II fractured reservoir had much higher original oil in place but might have a much lower recovery rate than the basement reservoir.

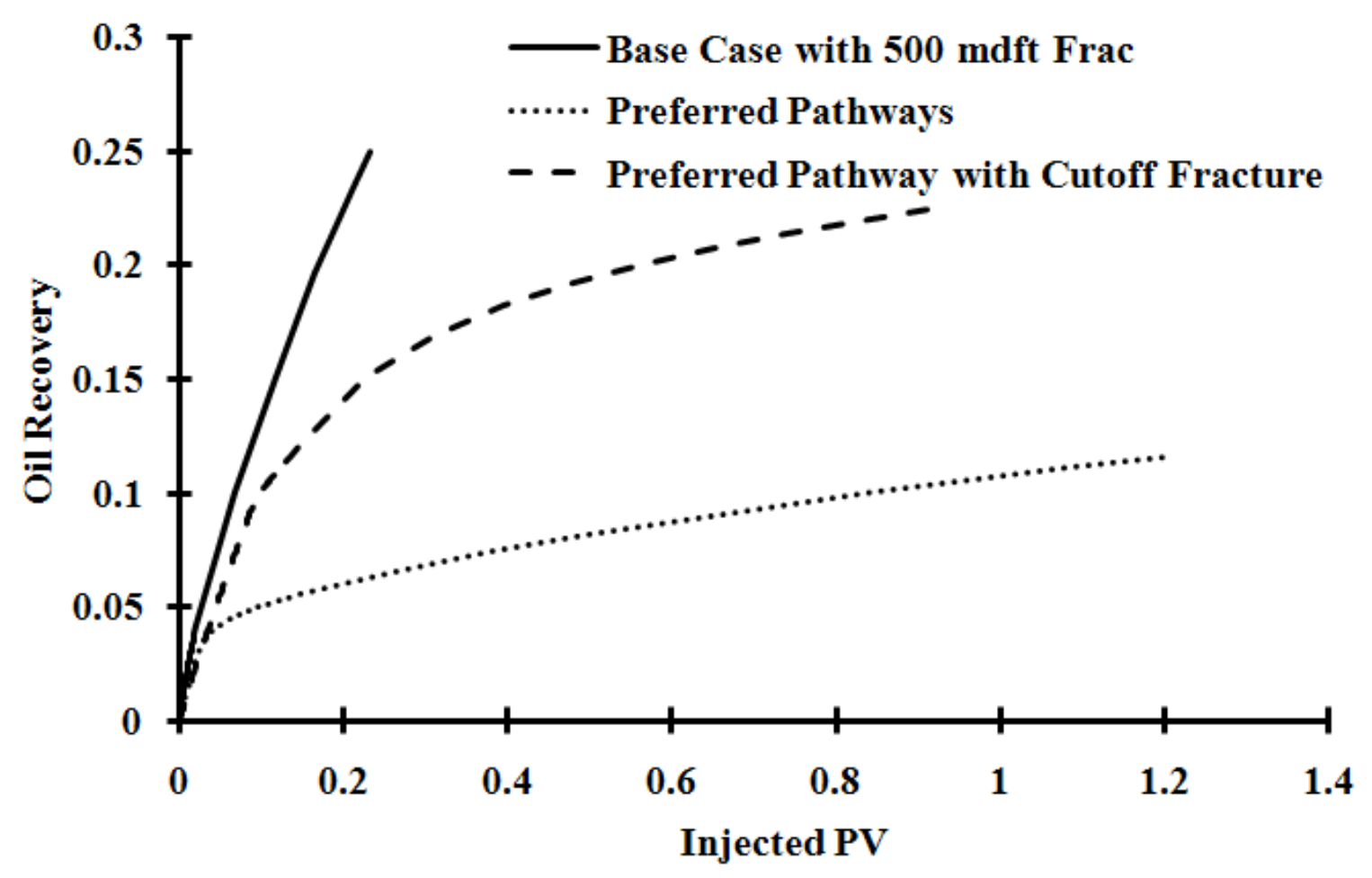

Figure 6.23 Dimensionless analysis of oil recovery vs. injection water

\section{Chapter Summary}

In this chapter, a new three-dimensional, three-phase, unstructured-grid black-oil simulator was introduced for a highly heterogeneous type II fractured reservoir study. A series of simulations with Type II reservoirs (reservoirs featuring both the matrix and the fractures) was performed primarily to demonstrate the capability of this in-house CVFE simulator. This simulator was good at directly calculating fluid flow through DFN modeled fractures/faults. At the beginning of the chapter, this CVFE Type II simulator was quantitatively verified with the reported core-flood data. The results were in good agreement in various conditions. The usefulness of using 
discrete-fracture models was discussed by looking at production from faulted-fractured systems. It was shown that given a certain reservoir and fracture description the simulator could be employed to optimize oil recovery.

\section{Chapter Nomenclature}

$\begin{array}{lll}\mathrm{a} \sim \mathrm{k} & = & \text { point identifying the tetrahedral control volume } \\ B & = & \text { formation volume factor } \\ & \text { (Subscripts } \mathrm{g}, \mathrm{o} \text { and w refer to gas, oil and water respectively) } \\ B_{r} & = & \text { formation volume factor at the reference pressure } \\ \mathrm{C} & = & \text { compressibility } \\ C_{B, \mu} & = & \text { compressibility of formation factor and viscosity, } 1 / \mathrm{psi} \\ e & = & \text { fracture/fault storage aperture, } \mathrm{ft} \\ g & = & \text { gas phase } \\ \mathbf{k} & = & \text { absolute permeability, md } \\ k_{r} & = & \text { relative permeability } \\ o & = & \text { oil phase } \\ p & = & \text { fluid pressure, psi } \\ q & = & \text { flux vector } \\ R_{S} & & \text { ghase sail ratio, MSCF/STB } \\ S & & \end{array}$

(Subscripts g, o and w refer to gas, oil and water respectively)

$S_{o r} \quad=\quad$ residual oil saturation

$u \quad=\quad$ velocity, $\mathrm{ft} /$ day

$w \quad=\quad$ water phase 


$$
\begin{array}{lll}
\emptyset & = & \text { porosity } \\
\rho & = & \text { density, } \mathrm{lbm} / \mathrm{ft}^{3} \\
\mu & = & \text { viscosity, } \mathrm{cp}
\end{array}
$$

\section{Chapter Bibliography}

Aavatsmark, I., Barkve, T. and Mannseth, T.: “Control Volume Discretization Methods for 3D Quadrilateral Grids in Inhomogeneous, Anisotropic Reservoirs”, paper SPE 38000, (1997) Araujo, H., Lacentre, P., Zapata, T., Del Monte, A., Dzelalija, F., Repsol-YPF; Gilman, J., Meng, H., Kazemi, H., Ozkan, E., "Dynamic Behavoir of Discrete Fracture Network (DFN) Models," paper SPE 91940 (2004)

Basquet, R., Cohen, C.E. and Bourbiaux, B., "Fracture Flow Property Identification: An Optimized Implementation of Discrete Fracture Network Models," paper SPE 93748 (2005)

Dean, R.H., and Lo, L.L.: "Simulations of Naturally Fractured Reservoirs," SPERE (May, 1988) 638-648

Dershowitz, B., LaPointe, P., Eiben, T., Wei, L., "Integration of Discrete Feature Network Methods with Conventional Simulator Approaches,” paper SPE 49069 (1998)

Dershowitz, W. S., “A Probablistic Model for the Deformability of Jointed Rock Masses” M.S. Thesis, Massachusetts Institute of Technology, Cambridge, MA (1979)

FRED User's Mannual, Golder Associate (2007)

Fu, Y., Yang, Y-K., and Deo, M., "Three-Dimensional, Three-Phase Discrete-Fracture Reservoir Simulator Based on Control Volume Finite Element (CVFE) Formulation”, paper SPE 93292, (2005)

Fung, L.S., Hiebert, A.D., and Nghiem, L.X., "Reservoir Simulation With a Control Volume Finite Element Method", paper SPE 21224 (1991)

Gong, B., Karimi-Frad, M., and Durlofsky, L.J., "An Upscaling Procedure for Constructing Generalized Dual-Porosity/Dual-Permeability Models from Discrete Fracture Characterizations", paper SPE 102491, (2006)

Kazemi, H. et al.: "Numerical Simulation of Water-Oil Flow in Naturally Fractured Reservoirs," SPEJ (Dec. 1976) 317-26.

Kim, J. and Deo, M.D., A Finite Element Discrete Fracture Model for Multiphase Flow in Porous Media, AIChE J. Vol 46, \#6, 1120 (2000) 
Kleppe, J. and Morse, R. A., "Oil production from fractured reservoirs by water displacement", paper SPE 5084 (1974)

Monteagudo, J.E.P. and Firoozabadi, A., Control Volume Method for the Numerical Simulation of Two-Phase Immiscible Flow in Two- and Three-dimensional Discrete Fracture Media, Water Resource Research, Vol. 40. (2004)

Pooladi-Darvish, M. and Firoozabadi, A., "Experiments and Modelling of Water Injection in Water-wet Fractured Porous Media", J. Can. Pet. Tech., vol. 39, no. 3 (2000)

Ronald A. Nelson, "Geologic Analysis of Naturally Fractured Reservoirs", Gulf Professional Publishing, second edition (2001)

Rossen, R.H.: "Simulation of Naturally Fractured Reservoirs with Semi-Implicit Source Terms," paper SPEJ (Dec. 1977) 201-10.

Verma, S. and Aziz, K.: "A Control Volume Scheme for Flexible Grids in Reservoir Simulation", paper SPE 37999, (1997)

Warren, J.E. and Root, P.J.: “The Behevior of Naturally Fractured Reservoir," SPEJ (September 1963).

Yamamoto, R. H., Ford, W.T., Padgett, J. B. and Boubeguira, A. "Compositional reservoir simulator for fissured systems - The single block model”, Petrol. Eng. J., 11, No.2, pp. 113-28 (1971)

Y. K. Yang, "Finite-Element Multiphase Flow Simulation". PhD thesis, University of Utah, Salt Lake City, UT, (2003) 


\section{Chapter 7. Development of the Compositional-Thermal Reservoir Simulator}

\section{Table of Contents}

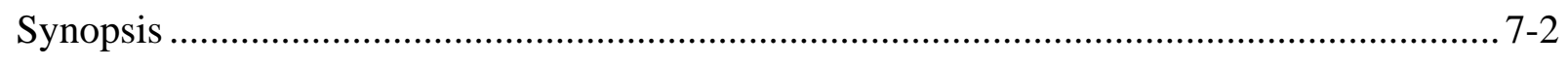

Background ..............................................................................................................

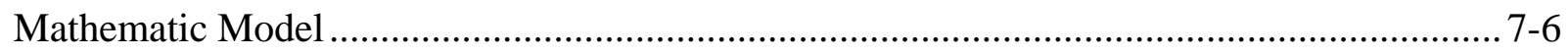

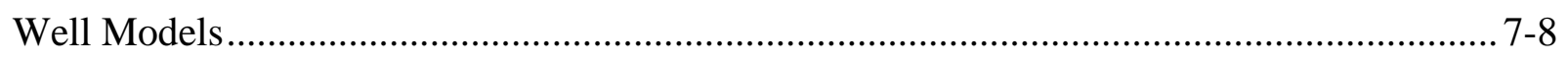

Solution Procedure and Variable Alignment .................................................................. 7-9

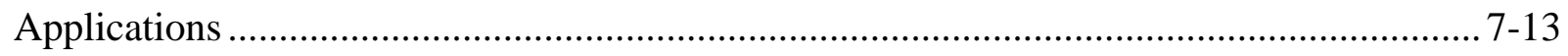

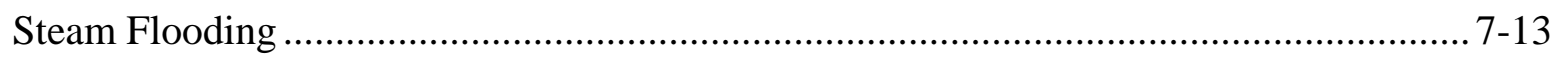

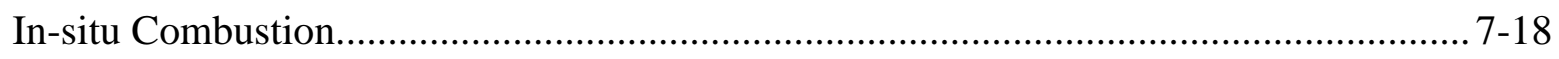

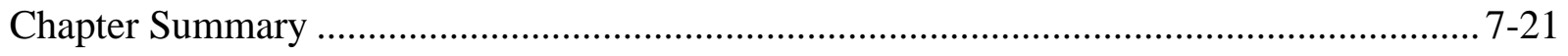

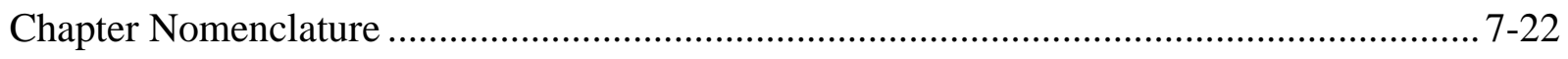

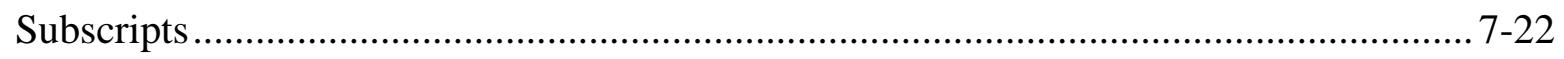

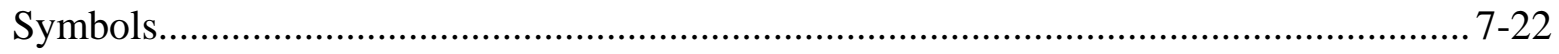

Chapter Bibliography …………………………………....... 7-Error! Bookmark not defined.

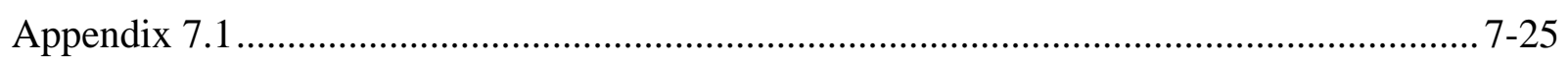

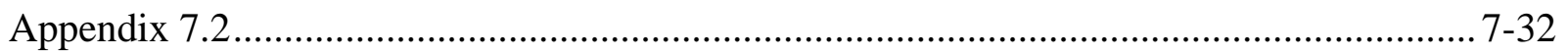




\section{Synopsis}

In a thermal-compositional reservoir simulator, pressures, saturations, temperature and compositions in all the existing phases must be solved. When this equation system is solved implicitly, a system of nonlinear equations results, which is solved by the Newton's method. This requires computation of the Jacobian matrix of partial derivatives. If the appropriate dependent variable is not lined-up with the correct equation, zero pivot elements are generated in the Jacobian matrix. This situation is complicated by the fact that new phases may appear, and existing phases may disappear leading to the type and number of dependent variables changing constantly. Elimination of these zero pivot elements using pivoting is computationally expensive for large matrix systems. In this chapter, we present a robust algorithm for lining-up the dependent variables and the equation system appropriately to avoid zero pivots in the Jacobian. This technique can be applied to the thermal-compositional model with any number of components and phases and there is no constraint on the number of thermal equilibrium relationships. In a system with Nc components and Np phases, and Ne thermal equilibrium constraints, $\mathrm{Nc}+\mathrm{Np}+\mathrm{Ne}+2$ dependent variables are lined with appropriate equations (pressure and temperature are the two additional variables). Any phase equilibrium calculation (K-value based, equation of state) can be used in this approach.

The motivation for equation line-up and some previous methods are presented followed by a description of the conservation and constraint equations in thermal modeling. A brief description of the simulator framework that allows coupling different physical models and discretization methods is provided. The rules of variable selection for avoiding zero pivots are discussed in detail, including a method to obtain analytical derivatives. As proof of concept, two applications are discussed: a steamflooding example in a fractured system, using the discrete-fracture 
modeling approach and in situ combustion. A control volume finite-element discretization is used in steamflooding while a finite-difference approach based on the Cartesian grid is employed in the in situ combustion application.

\section{Background}

Reservoir simulation requires solutions of nonlinear partial differential equations. The set of nonlinear equations that result when the differential equations are discretized are solved using the Newton's method, when an implicit solution procedure is employed. Among reservoir simulators, thermal simulators are the most complex. Solution of an energy balance equation is required along with component conservation equations. Pressures and saturations are additional variables. Writing a general thermal simulator, where phases appear and disappear, and where components get converted to new products, requires a general and robust approach for selecting how dependent variables get lined up for solution alongside the Jacobian matrix. If proper alignment is not achieved, zero pivot elements result and to overcome this, computationally expensive pivoting is necessary. Different variable alignment approaches have been proposed previously. Commonly used strategies for lining up variables with equations are summarized below. The vector $\overrightarrow{\mathrm{V}}$ refers to the dependent variables, while the vector $\overrightarrow{\mathrm{R}}$ points to the equations to be solved.

Coats et. al. (1980):

$\overrightarrow{\mathrm{V}}=\mathrm{v}\left\{\mathrm{S}_{1}, \ldots, \mathrm{S}_{\mathrm{Np}-1}, \mathrm{P}, \mathrm{X}_{\mathrm{Np}+1}, \ldots, \mathrm{T}\right\}$

$\overrightarrow{\mathrm{R}}=\mathrm{R}\left\{\mathrm{R}_{1}, \ldots, \mathrm{R}_{\mathrm{Nc}}, \mathrm{R}_{\mathrm{E}}\right\}$

Where, variable $\mathrm{X}_{\mathrm{i}}$ 's represent the mole fraction of component ' $\mathrm{i}$ ' in its “master phase”. The mole fraction of component ' $\mathrm{i}$ ' in phase $\mathrm{p}\left(\mathrm{x}_{\mathrm{p}, \mathrm{i}} \mathrm{s}\right)$ can be calculated by $\mathrm{x}_{\mathrm{p}, \mathrm{i}}=\mathrm{K}_{\mathrm{i}, \mathrm{p}} \mathrm{X}_{\mathrm{i}}$. $\mathrm{S}_{\mathrm{j}}$ is 
replaced by $\mathrm{X}_{\mathrm{j}}$ if phase ' $\mathrm{j}$ ' is absent. Mass conservation equation for the coke component is considered as a constraint equation.

Eclipes 300 (2006):

$$
\overrightarrow{\mathrm{v}}=\mathrm{v}\left\{\mathrm{m}_{1}, \ldots, \mathrm{m}_{\mathrm{Nc}-1}, \mathrm{~m}_{\mathrm{w}}, \mathrm{E}, \mathrm{P}\right\} \overrightarrow{\mathrm{R}}=\mathrm{R}\left\{\mathrm{R}_{1}, \ldots, \mathrm{R}_{\mathrm{Nc}-1}, \mathrm{R}_{\mathrm{w}}, \mathrm{R}_{\mathrm{E}}, \mathrm{R}_{\mathrm{v}}\right\}
$$

Where $R_{E}$ is the total internal energy and $R_{V}$ is the volume constraint equation computed by $R_{V}=V_{\text {pore }}-V_{\text {fluid }}=0$. With this approach, variables do not have to be realigned upon phase changes.

CMG(SXY option) STARS (2006):

$$
\begin{aligned}
& \vec{v}=v\left\{P, S_{o}, \ldots, x_{o c}, S_{g} y_{N_{C G}}, \ldots, S_{g} y_{N_{C G}}, \vec{R}=R\left\{R_{w}, R_{o_{1}}, \ldots, R_{o_{o c}}, \ldots, R_{N_{C G}}, \ldots, R_{N_{C G}}\right. \text {, The gas }\right. \\
& \left.\left.\mathrm{C}_{\mathrm{s}_{1}}, \ldots, \mathrm{C}_{\mathrm{s}_{\mathrm{ns}}}, \mathrm{T}\right\} \quad \mathrm{R}_{\mathrm{s}_{1}} \ldots, \mathrm{R}_{\mathrm{s}_{\mathrm{ns}}}, \mathrm{R}_{\mathrm{E}}\right\}
\end{aligned}
$$

molar constraint or the volume constraint is solved optionally depending on the flash algorithm and the equilibrium condition. $\quad$ For a wide-boiling system, the saturation constraint is used while the gas phase constraint is used in a narrow-boiling system. A series of rules are required to re-align equations and variables once phase change occurs.

Chen et. al. (2002):

$$
\overrightarrow{\mathrm{v}}=\mathrm{v}\left\{\mathrm{x}_{1}, \ldots \mathrm{x}_{\mathrm{Nc}-2}, \mathrm{P}, \mathrm{S}_{\mathrm{w}}, \mathrm{S}_{\mathrm{g}}\right\} \overrightarrow{\mathrm{R}}=\mathrm{R}\left\{\mathrm{R}_{1}, \ldots \mathrm{R}_{\mathrm{Nc}-1}, \mathrm{R}_{\mathrm{w}}, \mathrm{R}_{\mathrm{E}}\right\}
$$

In this case, $x_{i}=\sum_{p=1}^{N p} x_{p, i}$ is solved. $S_{g}$ is replaced by $T$ if the block is unsaturated.

Buchanan et. al. (1985):

$$
\overrightarrow{\mathrm{v}}=\mathrm{v}\left\{\mathrm{P}, \mathrm{z}_{2}, \ldots \mathrm{z}_{\mathrm{Nc}}, \mathrm{T}, \mathrm{F}_{\mathrm{g}}\right\} \overrightarrow{\mathrm{R}}=\mathrm{R}\left\{\mathrm{R}_{\mathrm{w}}, \mathrm{R}_{2}, \ldots, \mathrm{R}_{\mathrm{Nc}}, \mathrm{R}_{\mathrm{E}}, \mathrm{R}_{\mathrm{F}}\right\}
$$

Where, $\mathrm{R}_{\mathrm{F}}$ is the flash constraint and is aligned with gas fraction $\mathrm{F}_{\mathrm{g}}$. The approach required no re-alignment in phase changes. 
Grabowski et. al. (1979):

$$
\begin{aligned}
& \overrightarrow{\mathrm{V}}=\mathrm{v}\left\{\mathrm{P}, \mathrm{S}_{\mathrm{w}}, \mathrm{S}_{\mathrm{o}}, \mathrm{T}, \mathrm{x}_{\mathrm{g}, \mathrm{Ng}}, \mathrm{x}_{\mathrm{g}, \mathrm{No}+2}, \mathrm{x}_{\mathrm{g}, \mathrm{Ng}-1}, \mathrm{x}_{\mathrm{o}, 2} \overrightarrow{\mathrm{R}}=\mathrm{R}\left\{\mathrm{R}_{\mathrm{w}}, \mathrm{R}_{2}, \ldots, \mathrm{R}_{\mathrm{Nc} 01}, \mathrm{R}_{\mathrm{E}}\right\}\right. \\
& \left., \ldots \mathrm{x}_{\mathrm{o}, \mathrm{No}-1}\right\}
\end{aligned}
$$

The model could consider only one solid component (coke), and the coke conservation equation is solved explicitly.

$\underline{\text { Rubin et. al. (1985): }}$

Equation re-combination was required in this approach. The existence of non-condensable gas demanded a different algorithm to align the variables. Phase change also required variable realignment.

It is not convenient to implement analytical Jacobian using the techniques described previously. One of the primary motivations for developing the alignment procedure in this work was to be able to compute analytical Jacobian matrices. The thermal simulator described in this chapter was developed under the UFES (Utah Finite Element Simulator) modular framework. The oil reservoir simulator development in UFES is divided into two main modules: PhyiscalModel (PM) and DiscretizationMethod (DM). The DM module is responsible for computing the driving forces term $-\mathbf{k} \nabla \Phi$ for each flux so it is a discrete-method dependent module. The focus of this chapter is on the development of the thermal model in PM which controls the computation of the physical/chemical properties and the solution of the governing equations.

The key features of this thermal model enumerated below.

1) $P, S_{p}, X_{p}$, i and $T$ are used as variables and a novel alignment approach is proposed to prevent the occurrence of zero pivots in the Jacobian matrix.

2) Equation decoupling is used to separate the solution sequence of conservation equations and constraint equations. 
3) The number of components is a variable and is determined in input data. The components are allowed to be distributed in the oil, gas, water and solid phases depending on the component-phase distribution table.

4) The pore volume is occupied by oil, gas, water, solid phases (consisting of active solid components), and the inert components are considered to be part of the rock matrix.

5) The number and types of chemical reactions are determined in the input data file.

6) Concentration driven diffusion is assumed negligible.

7) The model is solved fully implicitly. The Jacobian matrix is computed analytically to improve the efficiency of the Newton's method.

The first three features generalize the mass conservation of the fluid and non-fluid components, and provide a flexible handle for solving the mass conservation equations. The last feature provides a high accuracy “searching slope” for Newton's method. The computation of analytical expressions for the Jacobian derivatives can easily be achieved because of the modularized design. The simulator can be used on applications such as steam flooding, in-situ conversion, and in-situ combustion. After minor modifications, the simulator can be extended to other applications such as polymer flooding and $\mathrm{CO}_{2}$ sequestration.

In this chapter, we emphasize the introduction of the solution procedure of the thermal model including the new equation line-up method, computation of the analytical Jacobian matrix and equation decoupling. Two applications, steam flooding in an unstructured grid domain and insitu combustion in the Cartesian domain, are used to show the adaptability of this new model.

\section{Mathematic Model}

For an Nc component and Np phase thermal recovery problem, there are Nc + 1 conservation equations to be solved: Nc mass balance equations and one energy balance equation. 
The mass balance equation of component ' $i$ ' is given by

$$
\sum_{\mathrm{p}=1}^{\mathrm{Np}} \mathrm{x}_{\mathrm{p}, \mathrm{i}} \rho_{\mathrm{p}} \nabla \cdot \overrightarrow{\mathrm{f}}_{\mathrm{p}}+\mathrm{q}_{\mathrm{i}}+\sum_{\mathrm{r}=1}^{\mathrm{Nr}} \mathfrak{R}_{\mathrm{r}, \mathrm{i}}=\frac{\partial}{\partial \mathrm{t}} \phi \sum_{\mathrm{p}=1}^{\mathrm{Np}} \mathrm{x}_{\mathrm{p}, \mathrm{i}} \mathrm{S}_{\mathrm{p}} \rho_{\mathrm{p}}
$$

For the reaction term, rate expressions of the power-law type $\mathfrak{R}_{r, \mathrm{i}}=\mathbf{a}_{\mathrm{r}, \mathrm{i}} \mathrm{k}_{\mathrm{r}} \prod_{\mathrm{j}=1}^{\mathrm{Nc}} \mathrm{C}_{\mathrm{j}}^{\mathrm{n}_{\mathrm{j}}}$ are used.

The energy balance equation is written as:

$$
\begin{aligned}
& \sum_{\mathrm{p}=1}^{\mathrm{Np}} \widehat{\mathrm{H}}_{\mathrm{p}} \rho_{\mathrm{p}} \nabla \cdot \overrightarrow{\mathrm{f}}_{\mathrm{p}}+\nabla \cdot \mathbf{k}_{\mathrm{c}} \nabla \mathrm{T}+\mathrm{Q}_{\mathrm{w}}+\mathrm{Q}_{\text {loss }}= \\
& \frac{\partial}{\partial \mathrm{t}} \phi \sum_{\mathrm{p}=1}^{\mathrm{Np}} \widehat{\mathrm{U}}_{\mathrm{p}} \mathrm{S}_{\mathrm{p}} \rho_{\mathrm{p}}+(1-\phi) \overline{\mathrm{U}}_{\mathrm{R}}
\end{aligned}
$$

The heat of reaction does not appear explicitly in the energy balance equation. The enthalpy differences in the reactant and product streams automatically account for the heats of reaction [9]. The heat of reaction has to be specified only if all reference enthalpies are zero.

In mass and energy conservation equations, the volumetric flux $\vec{f}_{p}$ for phase 'p' is computed by Darcy's flow equation

$$
\overrightarrow{\mathrm{f}}_{\mathrm{p}}=-\mathbf{k} \frac{\mathrm{kr}_{\mathrm{p}}}{\mu_{\mathrm{p}}}\left(\nabla \mathrm{P}_{\mathrm{p}}+\gamma_{\mathrm{p}} \nabla \mathrm{z}\right)
$$

For such a system, we can define the volume constraint

$$
\sum_{\mathrm{p}=1}^{\mathrm{Np}} \mathrm{S}_{\mathrm{p}}=1
$$

and Np phase constraints

$$
\sum_{i=1}^{N c} x_{p, i}=1
$$


The number of the equilibrium constraint equations depends on the number of equilibrium relationships defined by the user. There is a K-value equation for each component that partitions between two different phases.

$$
\frac{\mathrm{X}_{\mathrm{p}_{\mathrm{L}}, \mathrm{i}}}{\mathrm{X}_{\mathrm{pH}, \mathrm{i}}}=\mathrm{K}_{\mathrm{i}, \mathrm{p}_{\mathrm{L}}, \mathrm{p}_{\mathrm{H}}}
$$

The implicit form to represent all the conservation equations and constraint equations can be written as

$$
\begin{aligned}
& \mathrm{R}_{\mathrm{Ci}}=\mathrm{R}_{\mathrm{Ci}}\left(\mathrm{P}, \mathrm{S}_{\mathrm{p}}, \mathrm{x}_{\mathrm{p}, \mathrm{i}} \mathrm{T}\right) \\
& \mathrm{R}_{\mathrm{E}}=\mathrm{R}_{\mathrm{E}}\left(\mathrm{P}, \mathrm{S}_{\mathrm{p}}, \mathrm{x}_{\mathrm{p}, \mathrm{i}}, \mathrm{T}\right) \\
& \mathrm{R}_{\Sigma \mathrm{S}}=\mathrm{R}_{\Sigma \mathrm{S}}\left(\mathrm{S}_{\mathrm{p}}\right) \\
& \mathrm{R}_{\Sigma \mathrm{x}}=\mathrm{R}_{\Sigma \mathrm{x}}\left(\mathrm{x}_{\mathrm{p}, \mathrm{i}}\right) \\
& \mathrm{R}_{\mathrm{Kk}}=\mathrm{R}_{\mathrm{Kk}}\left(\mathrm{P}, \mathrm{x}_{\mathrm{p}, \mathrm{i}}, \mathrm{T}\right)
\end{aligned}
$$

In above equation set, $\mathrm{i} \in[1, \mathrm{Nc}], \mathrm{p} \in[1, \mathrm{~Np}]$ and $\mathrm{k} \in[1, \mathrm{Ne}]$. The equations to compute the properties in each equation are summarized in the Appendix.

\section{Well Models}

Volumetric flow of phase 'p' between the well node and control-volume ' ' can be computed by

$$
\mathrm{q}_{\mathrm{p}, \mathrm{l}}=\mathrm{PI} \xi_{\mathrm{p}}\left(\mathrm{P}_{\mathrm{wb}}-\mathrm{P}_{\mathrm{p}, \mathrm{l}}\right)
$$

For production well, $\xi_{p}=\frac{k_{p}}{\mu_{p}}$ is the relative mobility for phase 'p' computed based on the condition of the production block. For the injection well, total mobility $\xi_{T}=\sum_{p=1}^{N p} \frac{k r_{p}}{\mu_{p}}$ is used..

The well index $\mathrm{PI}_{\imath}$ is generally computed by 


$$
\mathrm{PI}_{\mathrm{\imath}}=\frac{2 \pi \cdot h \cdot \mathrm{k}_{\perp \mathrm{w}} \cdot \mathrm{f}_{\mathrm{h}} \cdot \mathrm{f}}{\ln \frac{\mathrm{r}_{\mathrm{e}}}{\mathrm{r}_{\mathrm{wb}}}+\mathrm{S}}
$$

In the Cartesian grid, $\mathrm{r}_{\mathrm{e}}$ is computed by

$$
\mathrm{r}_{\mathrm{e}}=\frac{2 \cdot \text { geofac }}{\sqrt{\pi}} \frac{\sqrt{\sqrt{\mathrm{k}_{\mathrm{yy}} / \mathrm{k}_{\mathrm{xx}}}(\Delta \mathrm{x})^{2}+\sqrt{\mathrm{k}_{\mathrm{xx}} / \mathrm{k}_{\mathrm{yy}}}(\Delta \mathrm{y})^{2}}}{\left(\mathrm{k}_{\mathrm{yy}} / \mathrm{k}_{\mathrm{xx}}\right)^{1 / 4}+\left(\mathrm{k}_{\mathrm{xx}} / \mathrm{k}_{\mathrm{yy}}\right)^{1 / 4}}
$$

For the unstructured grid, one option to compute $r_{e}$ is

$$
r_{e}=\left[V_{\imath} \frac{3}{4 \pi}\right]^{1 / 3}
$$

$\mathrm{V}_{\imath}$ is the volume of the control-volume ' $\mathrm{l}$ ' where the well is completed. This option has been implemented in the Control Volume Finite Element (CVFE) model discussed later in the chapter. Vinsome et. al. (1980) suggest a semi-analytical model to compute the heat loss to the surrounding rock. The model requires no information from internal gridding so it is directly implemented without any modification in both the Cartesian grid and the unstructured grid model.

\section{Solution Procedure and Variable Alignment}

The variables $\overrightarrow{\mathrm{v}}=\left\{\mathrm{P}, \mathrm{S}_{\mathrm{p}}, \mathrm{X}_{\mathrm{p}, \mathrm{i}}, \mathrm{T}\right\}$ in the non-linear equation set are updated by $\overrightarrow{\mathrm{v}}^{\mathrm{t}+1}=\overrightarrow{\mathrm{v}}^{\mathrm{t}}+\delta \overrightarrow{\mathrm{v}}$ at each new time step where $\delta \overrightarrow{\mathrm{v}}$ is computed by the Newton's method.

$$
\overrightarrow{\mathrm{R}}=-\left[\frac{\partial \mathrm{R}}{\partial \mathrm{v}}\right] \delta \overrightarrow{\mathrm{v}}
$$

Analytical Jacobian derivatives $\left[\frac{\partial \mathrm{R}}{\partial \mathrm{v}}\right]$ are usually computed by applying a series of chain rules. 


$$
\begin{aligned}
& \frac{\partial \mathrm{R}}{\partial \mathrm{v}}=\frac{\partial \mathrm{R}}{\partial \mathrm{P}} \frac{\partial \mathrm{P}}{\partial \mathrm{v}}+\frac{\partial \mathrm{R}}{\partial \mathrm{T}} \frac{\partial \mathrm{T}}{\partial \mathrm{v}} \\
& +\sum_{\mathrm{p}=1}^{\mathrm{Np}}\left(\frac{\partial \mathrm{R}}{\partial \mathrm{S}_{\mathrm{p}}} \frac{\partial \mathrm{S}_{\mathrm{p}}}{\partial \mathrm{v}}+\sum_{\mathrm{i}=1}^{\mathrm{Nc}} \frac{\partial \mathrm{R}}{\partial \mathrm{x}_{\mathrm{p}, \mathrm{i}}} \frac{\partial \mathrm{x}_{\mathrm{p}, \mathrm{i}}}{\partial \mathrm{v}}\right)
\end{aligned}
$$

$\frac{\partial \mathrm{P}}{\partial \mathrm{v}}, \frac{\partial \mathrm{S}_{\mathrm{p}}}{\partial \mathrm{v}}, \frac{\partial \mathrm{x}_{\mathrm{p}, \mathrm{i}}}{\partial \mathrm{v}}, \frac{\partial \mathrm{T}}{\partial \mathrm{v}}$ are called unit derivatives which are either unity or zero. The derivatives of phase pressure require special care and are explained in the Appendix.

Zero pivots in the Jacobian matrix make it singular and must be avoided. The strategy to make this happen is to align each variable to the equation which is a function of that variable. For example, the volume constraint equation $R_{\Sigma s}$ can only be aligned to $S_{p}$. Aligning $R_{\Sigma s}$ to any variable other than $S_{p}$ causes the derivative $\left.\frac{\partial R_{\Sigma s}}{\partial v}\right|_{v \neq S_{p}}=0$.

The equations and the variables are aligned using a series of rules. As a result, the Jacobian matrix does not contain any zero pivots.

The residual function vector $\overrightarrow{\mathrm{R}}$ is organized in the following order.

1) Component mass conservation equations (oleic components, gaseous components, aqueous components, solid components)

2) Energy conservation equation

3) Volume constraint equation

4) Phase constraints (oil, gas, water, solid)

5) Thermal equilibrium constraints (oleic components, gaseous components, aqueous components, solid components)

A generalized component numbering scheme is shown in Table 7.1. Also shown in this table is the way components partition between the different (four) phases. 
Table 7.1 Numbering of components and their distribution in various phases

\begin{tabular}{|c|c|c|c|c|c|}
\hline Component ID & Type & Oil & Gas & Water & Solid \\
\hline $\begin{array}{l}\text { Oleic phase Comps } \\
1 \text { to NO }\end{array}$ & Oleic & $\begin{array}{l}\checkmark \\
1 \\
\checkmark\end{array}$ & $\begin{array}{l}\checkmark \\
\text { I } \\
\checkmark\end{array}$ & & \\
\hline $\begin{array}{l}\text { Aq. phase Comps } \\
\qquad \mathrm{NO}+1 \text { to } \mathrm{NO}+\mathrm{NW}\end{array}$ & Aqueous & & $\begin{array}{l}\checkmark \\
\text { I } \\
\checkmark\end{array}$ & $\begin{array}{l}\checkmark \\
1 \\
\checkmark\end{array}$ & \\
\hline Solid Phase Comps & & $(\checkmark)$ & $(\vee)$ & $(\checkmark)$ & $\checkmark$ \\
\hline $\begin{array}{c}\mathrm{NO}+\mathrm{NW}+1 \text { to } \\
\mathrm{NO}+\mathrm{NW}+\mathrm{NS}\end{array}$ & Solid & $\begin{array}{l}\text { I } \\
(\checkmark)\end{array}$ & $\begin{array}{l}\text { I } \\
(\checkmark)\end{array}$ & $\begin{array}{l}\text { I } \\
(\vee)\end{array}$ & I \\
\hline
\end{tabular}

After the order of the residual functions is decided, the equations are aligned with the variables by observing the following algorithm:

1) Do not solve $R_{\Sigma s}$ if only one phase exists.

1) Do not solve $R_{K k}$ if the component or the phase does not exist.

2) Do not solve $R_{C i}$ if component ' $i$ ' does not exist.

3) Align the first available $S_{P}$ to $R_{\Sigma s}$.

4) $\mathrm{R}_{\Sigma \mathrm{x}}$ 's are aligned with available $\mathrm{x}_{\mathrm{p}, \mathrm{i}}$. While doing this, ' $\mathrm{p}$ ' = 'i' condition get higher priority. For example, $\mathrm{x}_{1,1}$ is aligned with the $\mathrm{R}_{\Sigma \times 1}$.

5) $R_{K k}$ 's are aligned with available $x_{p, i}$ (align ' $i$ ' index, if no $x_{p, i}$ is available, using $T$ as the dependent variable).

6) Align remaining $x_{p, i}$ to $R_{C i}$ based on the $i$ index.

7) Align remaining $S_{p}$ to not-yet-aligned $R_{C i}$ (check if component ' $i$ ' exists in phase 'p'). If $R_{C i}$ 's are not available, use the $R_{E}$. 
8) Align $P$ to remaining $R_{C i}$ 's.

9) If $T$ is still available, align it with the $R_{E}$.

In each iteration step, phase change is handled by the following procedure.

For $\mathrm{S}_{\mathrm{p}}<0$ :

Phase 'p' disappears, $\mathrm{S}_{\mathrm{p}}$ and all corresponding $\mathrm{x}_{\mathrm{p}, \mathrm{I}}$ 's are set to zero.

For $S_{p}=0$ :

$\sum_{\mathrm{i}=1}^{\mathrm{Nc}} \mathrm{x}_{\mathrm{p}, \mathrm{i}}$ is monitored. Once the summation is $\geq 1$, the phase reappears. A small initial guess, for example, $\left(10^{-6}\right)$ is set to $S_{p}$ and all corresponding $x_{p, i}$ 's are updated according to the results from the flash calculation.

The Jacobian matrix is populated in sub-blocks as shown.

$$
\begin{aligned}
\left(\begin{array}{l}
\mathrm{R}_{\boldsymbol{p}} \\
\mathrm{R}_{\boldsymbol{s}}
\end{array}\right)_{\mathrm{I}}= & -\left[\begin{array}{ll}
\mathrm{A} & \mathrm{B} \\
\mathrm{C} & \mathrm{D}
\end{array}\right]_{\mathrm{I}, \mathrm{I}}\left(\begin{array}{l}
\delta \mathrm{v}_{\boldsymbol{P}} \\
\delta \mathrm{v}_{\boldsymbol{s}}
\end{array}\right)_{\mathrm{I}} \\
& -\sum_{\mathrm{J}}^{\mathrm{I} \neq \mathrm{J}}\left[\begin{array}{cc}
\mathrm{A} & \mathrm{B} \\
0 & 0
\end{array}\right]_{\mathrm{I}, \mathrm{J}}\left(\begin{array}{l}
\delta \mathrm{v}_{\boldsymbol{p}} \\
\delta \mathrm{v}_{\boldsymbol{s}}
\end{array}\right)_{\mathrm{J}}
\end{aligned}
$$

where A, B, C and D represent $\frac{\partial \mathrm{R}_{\boldsymbol{p}}}{\partial \mathrm{v}_{\boldsymbol{p}}}, \frac{\partial \mathrm{R}_{\boldsymbol{p}}}{\partial \mathrm{v}_{\boldsymbol{s}}}, \frac{\partial \mathrm{R}_{\boldsymbol{s}}}{\partial \mathrm{v}_{\boldsymbol{p}}}$ and $\frac{\partial \mathrm{R}_{\boldsymbol{s}}}{\partial \mathrm{v}_{\boldsymbol{s}}}$, respectively. The subscripts ${ }_{\boldsymbol{P}}$ and $\boldsymbol{s}$ represent primary (conservation) and secondary (constraint), equations respectively. Sub-block C and D are located only on the diagonal; therefore, this system can be decoupled by the Gaussian elimination technique. After the decoupling, the primary part and the secondary part can be solved separately. 


$$
\begin{aligned}
& -\left(\mathrm{R}_{\boldsymbol{P I}}-\sum_{J} \mathrm{~B}_{\mathrm{I}, \mathrm{J}} \mathrm{D}_{\mathrm{J}}^{-1} \mathrm{R}_{\boldsymbol{s} \mathrm{J}}\right)= \\
& \sum_{\mathrm{J}}\left[\mathrm{A}_{\mathrm{I}, \mathrm{J}}-\mathrm{B}_{\mathrm{I}, \mathrm{J}} \mathrm{D}_{\mathrm{J}}^{-1} \mathrm{C}_{\mathrm{J}}\right] \cdot\left(\delta \mathrm{v}_{\boldsymbol{P I}}\right) \\
& \left(\delta \mathrm{v}_{\boldsymbol{s} \mathrm{J}}\right)=-\mathrm{D}_{\mathrm{I}}^{-1}\left(\mathrm{C}_{\mathrm{I}}\left(\delta \mathrm{v}_{\boldsymbol{P I}}\right)+\mathrm{R}_{\boldsymbol{s} \mathrm{I}}\right)
\end{aligned}
$$

The computation becomes more efficient since a smaller system (primary part) is solved by the linear solver. The GMRES iterative solver was used for all the cases in this chapter. The solver and ILU preconditioner were provided by AztecOO package in the Trilinos project.

\section{Applications}

\section{Steam Flooding}

Steam flooding simulation was performed with the discrete fracture reservoir model (DFM) shown in Figure 7.1 with explicit fracture representation.

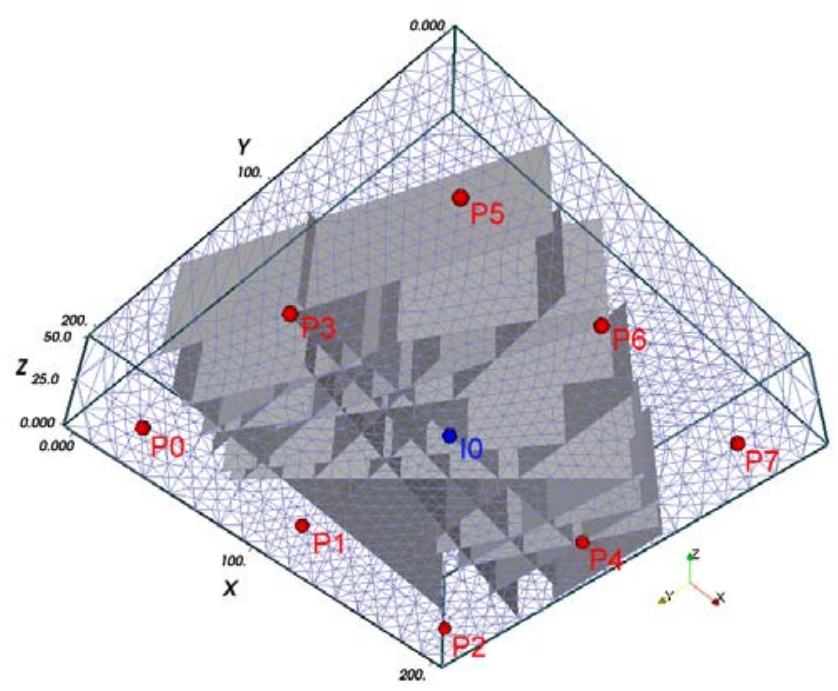

Figure 7.1 Steam flooding in an inverted nine-spot pattern in a fractured reservoir. Blue dot: injection well, red dots: production wells. 
The model consists of 5868 nodes, 28469 tetrahedral elements for representing the matrix and 3355 triangular elements for fractures. The model considered three components: heavy oil, light oil and water. The component distribution in this model is shown in Table 7.2 and data related to this model are given in Appendix 7.1 in Table A7.1. There were two sets of fractures in the simulation domain; one in the north-south direction and other in the east-west direction. A full scale inverted nine-spot (injection well at the center surrounded by eight producers) scheme was modeled. The impact of two different fracture/matrix permeability contrasts (2 and 100) on oil production was examined. The injection well and three production wells P3, P4 and P6 were located on fractures (see Figure 7.1).

Results of temperature, saturations of oil and water on $2000^{\text {th }}$ day for the high permeability contrast and the low permeability contrast are shown in Figures 7.2, 7.3 and 7.4. The oil was completely expelled from the swept zone. In the low contrast case, the swept zone is nearly uniform in every direction since the fractures do not have a significant impact. In high contrast case, the permeability of fracture is significantly larger compared with the matrix. Therefore, the flow is dominated by the fracture network. The production rates in P3, P4 and P6 are higher (see the oil production rate in Figure 7.5 and water saturation in Figure 7.4 b) and the breakthrough times are earlier. 
Table 7.1 Numbering of components and their distribution in various phases

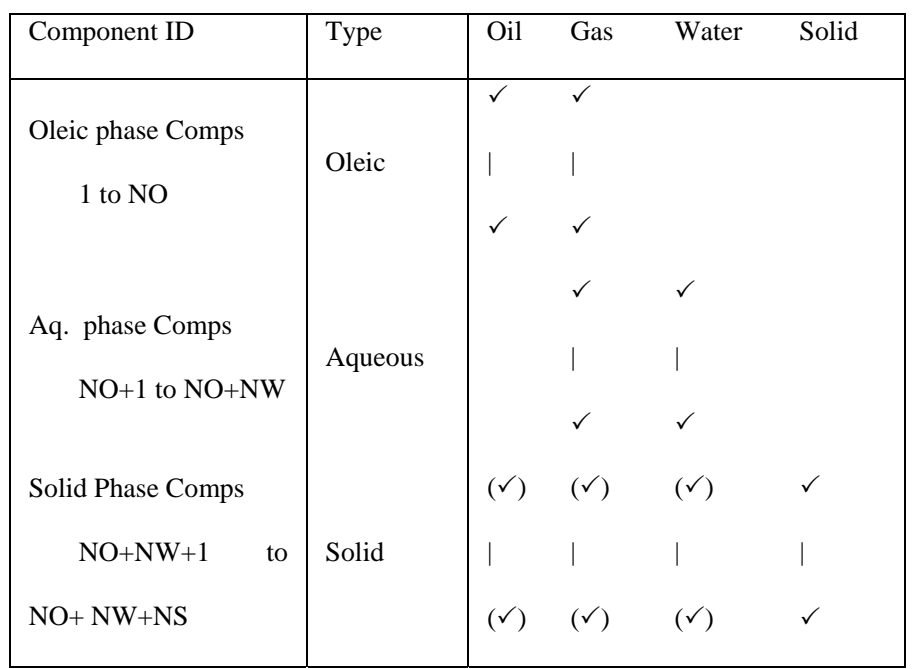

Table 7.2: Component-phase partition in steam flooding

\begin{tabular}{|l|l|lll|}
\hline Component ID & Type & Oil & Gas & Water \\
\hline Heavy oil & Oleic & $\checkmark$ & $\checkmark$ & \\
Light oil & Oleic & $\checkmark$ & $\checkmark$ & \\
Water & Aqueous & & $\checkmark$ & $\checkmark$ \\
\hline
\end{tabular}

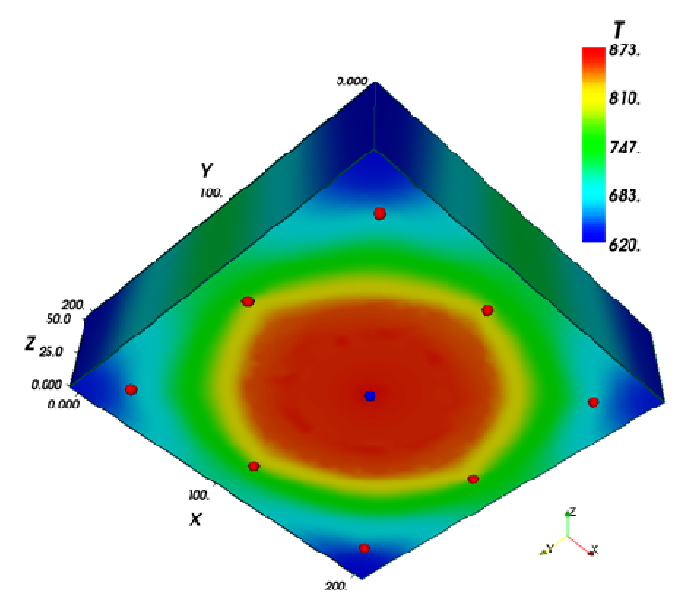

(a)

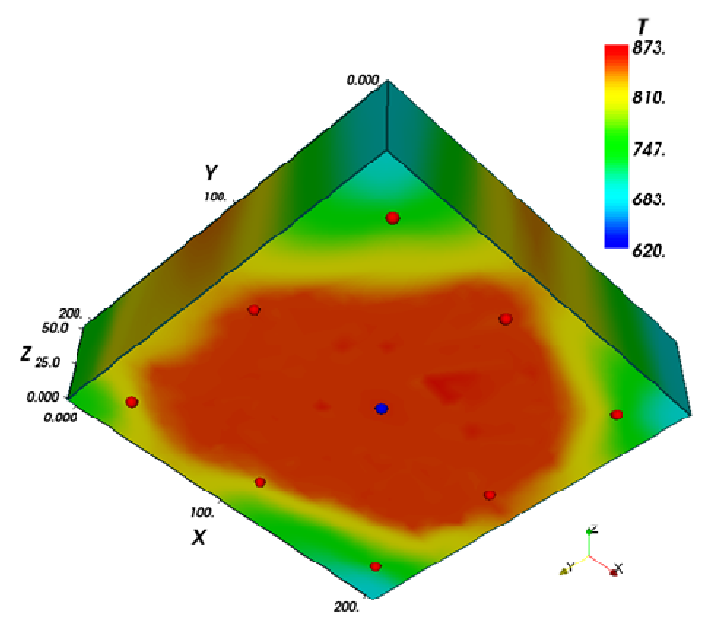

(b)

Figure 7.2 Temperature profiles of steam flooding in fractured reservoir (time $=2000$ days). a. Fracture-matrix permeability contrast $=2$; b. Fracture-matrix permeability contrast $=100$ 


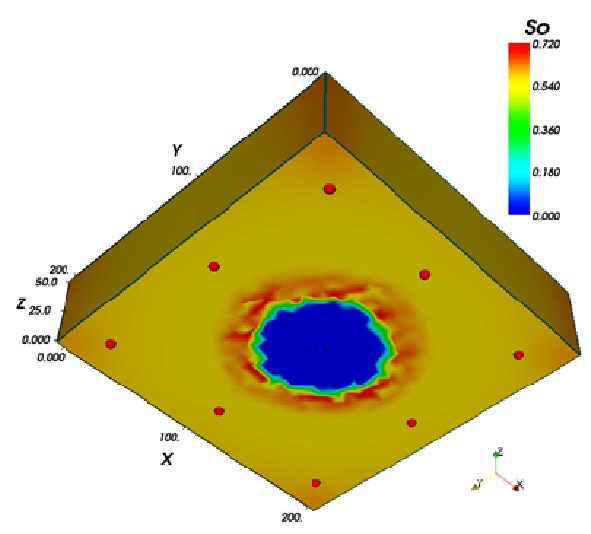

(a)

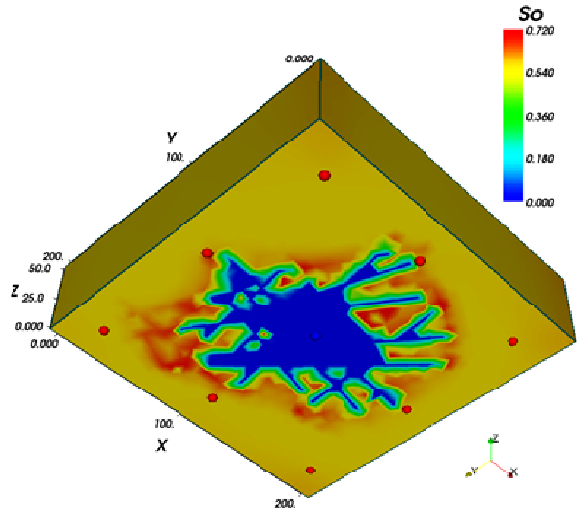

(b)

Figure 7.3 Oil saturation profiles of steam flooding in fractured reservoir (time $=2000$ days). a. Fracture-matrix permeability contrast $=2$; b. Fracture-matrix permeability contrast $=100$

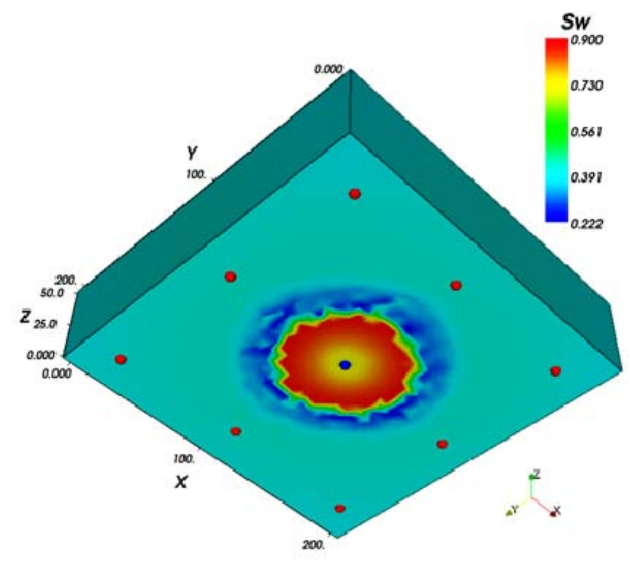

(a)

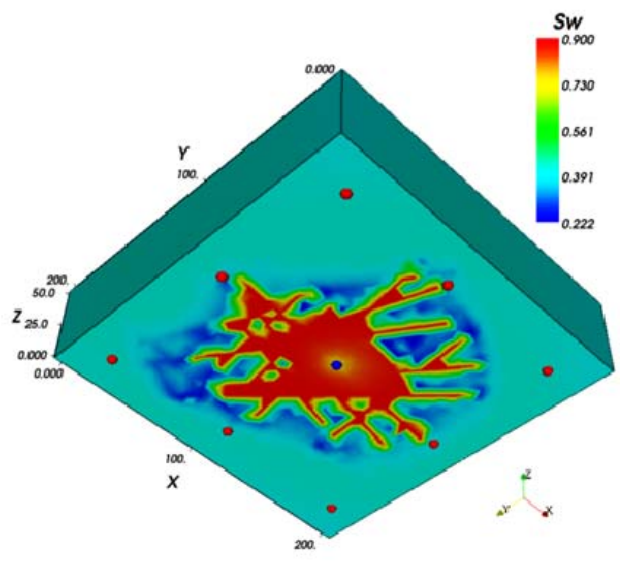

(b)

Figure 7.4 Temperature profiles of steam flooding in fractured reservoir (time $=2000$ days). a. Fracture-matrix permeability contrast $=2$; b. Fracture-matrix permeability contrast $=100$ 


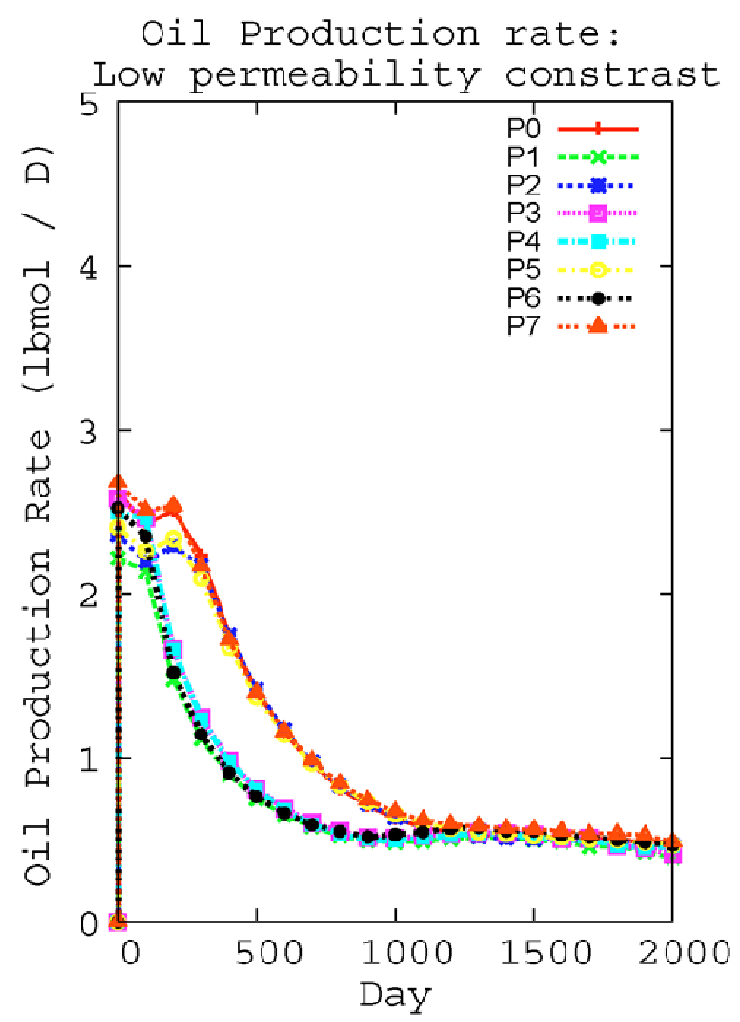

(a)

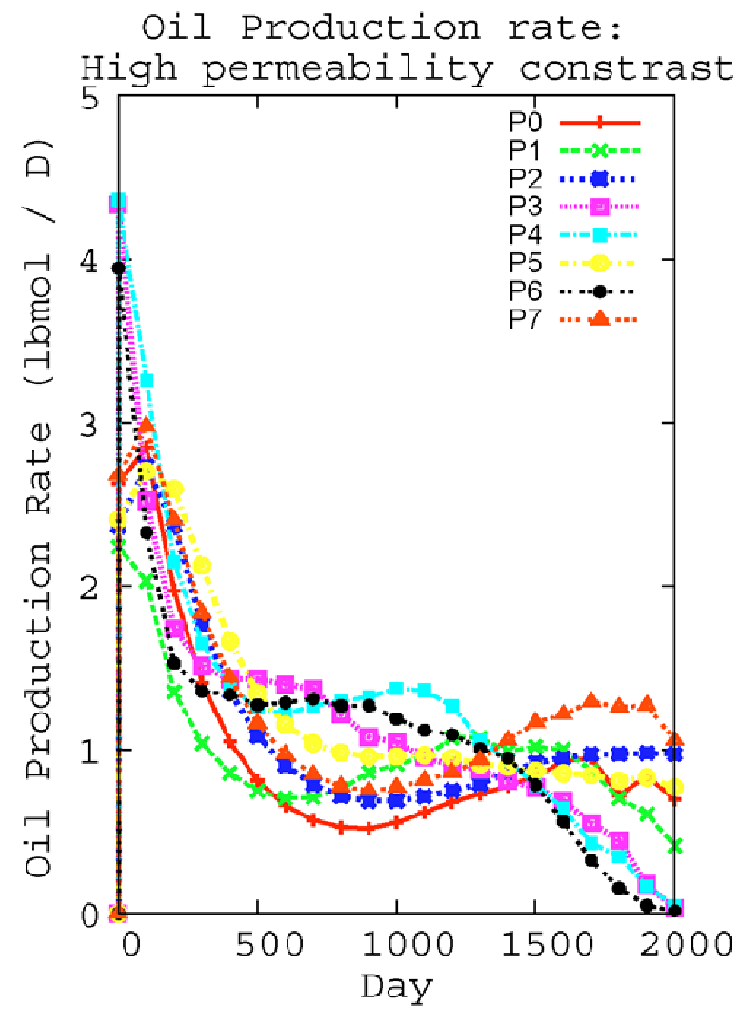

(b)

Figure 7.5 Oil production rate in steam flooding: a. Low permeability contrast; b. High permeability contrast 


\section{In-situ Combustion}

Thermal module was integrated with the Cartesian coordinate finite difference DM module for modeling a quarter size of a five-spot in-situ combustion process. A $10 \times 10 \times 5$ grid shown in Figure $7.6(10 \mathrm{ft} \times 10 \mathrm{ft} \times 5 \mathrm{ft}$ for each block) was used to simulate a thick, uniform formation containing heavy oil, light oil, water and coke. The air was injected at one corner and oil is produced at the opposite corner. Both the wells were completed in the bottom layer. Four chemical reactions were considered in this model. $\mathrm{CO}_{2}$ and $\mathrm{N}_{2}$ were lumped together as one inert gas component. The component phase distribution is shown in Table 7.4 and the data related to this model are given in Table 7.5. The gas components can dissolve into the oil phase. Coke component existed originally and can either be regenerated by cracking reaction from heavy oil or be consumed in coke oxidation reaction. The blocks around the injection well were set to $800 \mathrm{R}$ initially to simulate the ignition. Dry injection and varied amounts of water coinjection were compared. Water-air co-injections were continued from $20^{\text {th }}$ to $25^{\text {th }}$ day. The heats of reactions were provided in the input data since the reference enthalpies for all the $n$ components were set to zero.

The results of temperature, oil and water saturations at the $40^{\text {th }}$ day are shown in Figures 7.7, 7.8 and 7.9. The average temperature is lower in the combusted zone in the water co-injection case because the water evaporated in this zone and carried the heat toward the production well. The water recondensed beyond the combustion front and released the latent heat to heat up the reservoir (see water recondensation in Figure 7.9). Therefore, the oil bank (see Figure 7.8) in water-coinjection had moved farther away from the combustion front. The increase in the oil production rate due to this phenomenon is observed in Figure 7.10 for the wet combustion cases. 

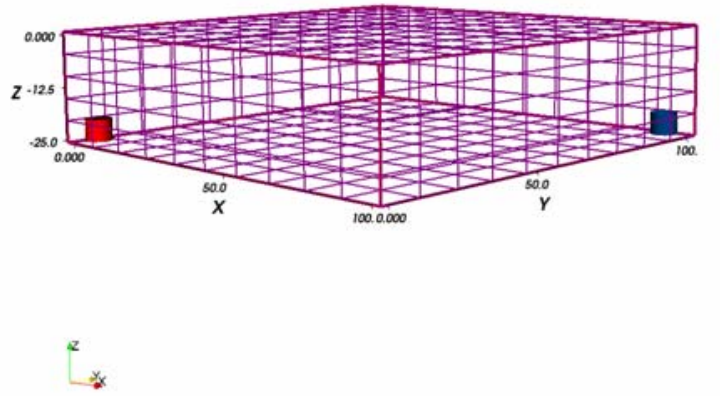

Figure 7.6 Domain of 1/4 five spot in-situ combustion simulation. Blue cylinder: production well, red cylinder: injection well.

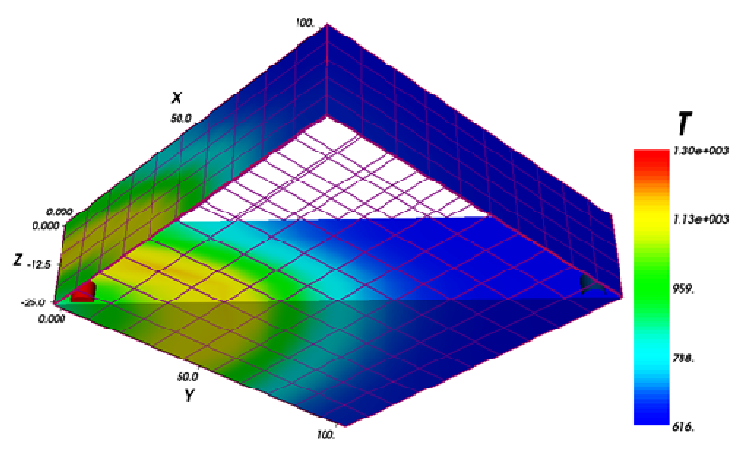

(a)

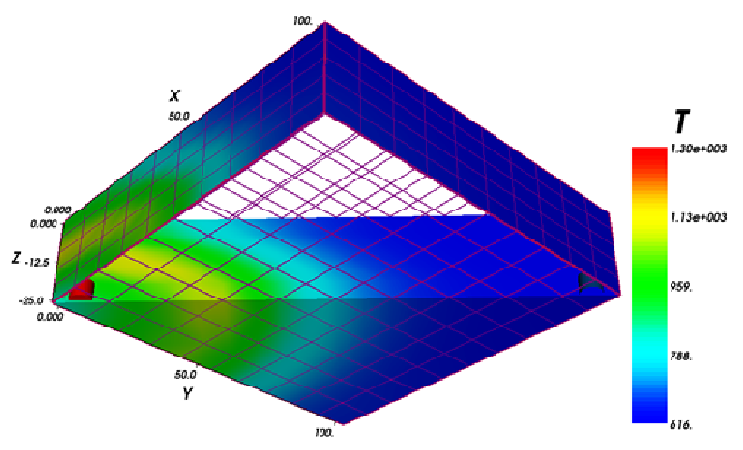

(b)

Figure 7.7 Temperature profiles in in-situ combustion (time = 40 days); a. Dry combustion; b. air to water ratio of $80: 20$ 


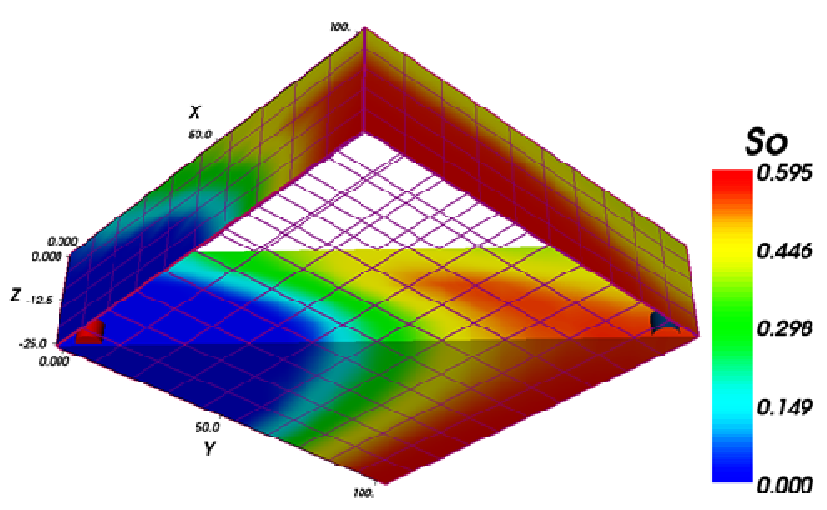

(a)

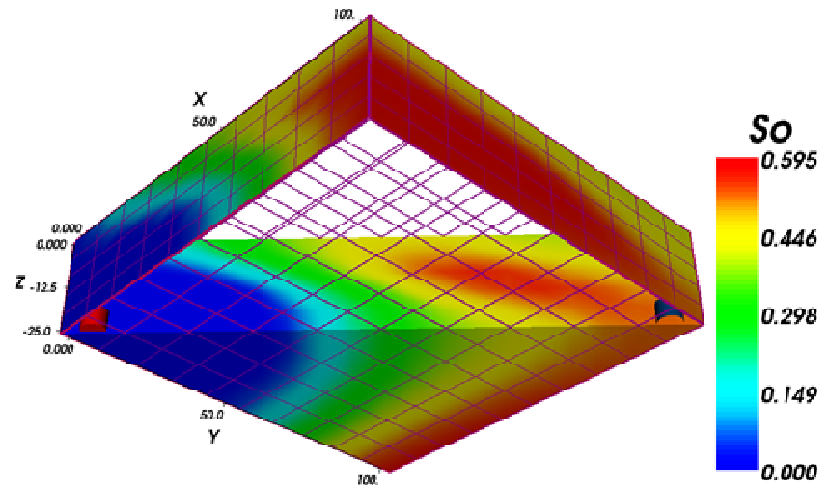

(b)

Figure 7.8 Oil saturation profiles in in-situ combustion (time $=40$ days); a. Dry combustion; b. air to water ratio of 80:20

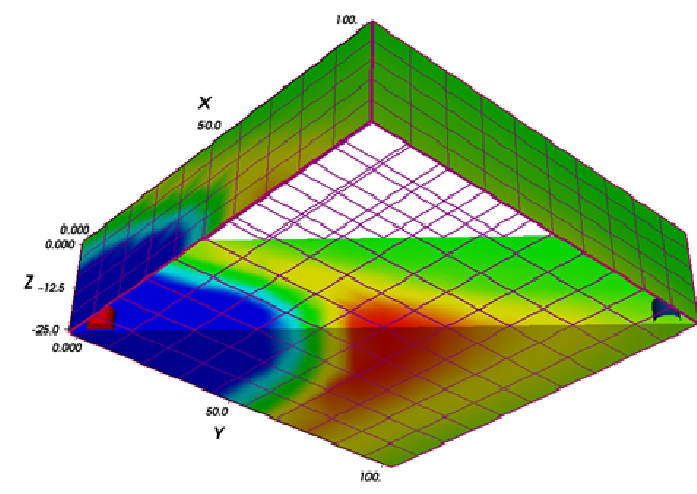

(a)
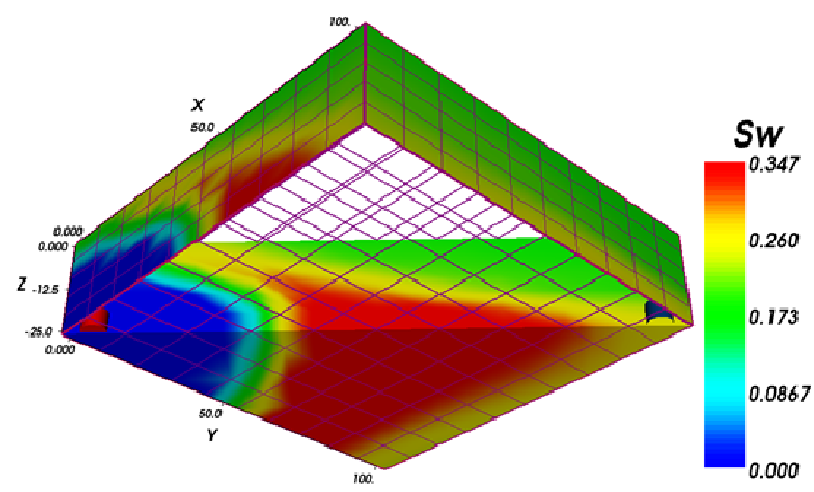

(b)

Figure 7.9: Water saturation profiles in in-situ combustion (time $=40$ days); a. Dry combustion; b. air to water ratio of $80: 20$ 


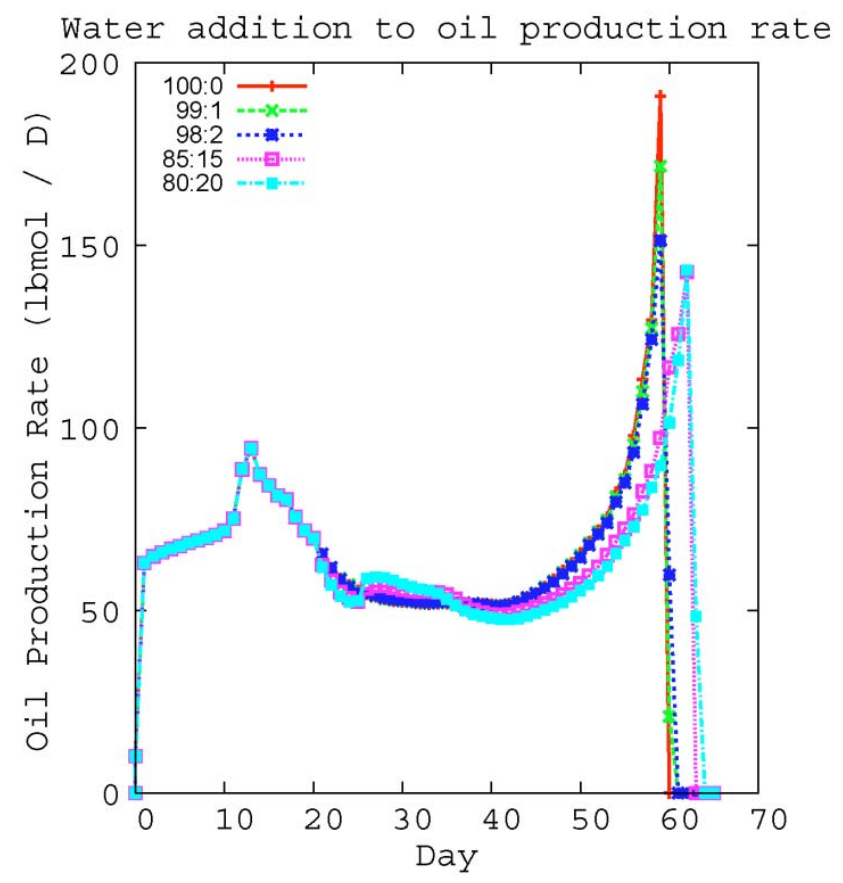

Figure 7.10 Oil production rate in in-situ combustion

\section{Chapter Summary}

The development of a fully implicit, general compositional thermal model using a new equation line-up concept was presented in this chapter. The new equation line-up procedure allows for computations of analytical Jacobian matrices. A study of two different applications, one with the control-volume finite element discretization and the other with finite difference showed that a given physical model can be integrated with different "DiscretizationMethod" modules to solve the reservoir problem. Incorporation of a discrete-fracture representation provides an alternative to dual porosity models for simulating thermal recovery in fractured reservoirs. Possible applications include modeling naturally fractured reservoirs and hydraulically fractured wells. 


\section{Chapter Nomenclature}

\section{Subscripts}

\begin{tabular}{|c|c|}
\hline 0 & Reference state \\
\hline $\mathrm{C}_{\mathrm{i}}$ & Index of residual function of conservation equation of component $i$ \\
\hline $\mathrm{R}$ & Rock \\
\hline $\mathrm{v}$ & Index of residual function of volume constraint equation \\
\hline E, F & $\begin{array}{l}\text { Index of residual function of energy conservation equation, flash } \\
\text { constraint }\end{array}$ \\
\hline $\mathrm{O}, \mathrm{W}, \mathrm{g}, \mathrm{s}, \mathrm{l}$ & Oil, water, gas, solid and liquid phase \\
\hline $\mathrm{p}, \mathrm{i}, \mathrm{r}$ & Phase index, component index and reaction index \\
\hline IG & Ideal gas \\
\hline $\mathrm{NCG}_{\mathrm{i}}$ & $\mathrm{i}^{\text {th }}$ non-condensable gas component \\
\hline $\mathrm{I}, \mathrm{J}$ & Control-volume index \\
\hline 1 & Layer index \\
\hline $\mathrm{K}_{\mathrm{k}}$ & $\begin{array}{l}\text { Index of residual function of conservation equation of } \mathrm{k}_{\mathrm{th}} \text { thermal } \\
\text { constraint equation }\end{array}$ \\
\hline $\mathrm{N}_{\mathrm{g}}, \mathrm{N}_{\mathrm{o}}$ & Number of component in gas phase, oil phase \\
\hline $\mathrm{O}_{\mathrm{g}}, \mathrm{O}_{\mathrm{w}}$ & Phase pair for oil and gas, oil and phase p, oil and water \\
\hline $\boldsymbol{P}, \boldsymbol{S}$ & Primary and secondary \\
\hline $\mathrm{wb}$ & Well bore \\
\hline
\end{tabular}

\section{Symbols}

$\begin{array}{ll}\mathrm{C}_{\mathrm{p}}, \mathrm{C}_{\mathrm{t}_{1}}, \mathrm{c}_{\mathrm{t}_{2}} & \text { Coefficient for molar density } \\ \mathrm{Cl}_{0}, \mathrm{cl}_{1} & \text { Coefficient for liquid phase viscosity } \\ \mathrm{Cg}_{0}, \mathrm{cg}_{1} & \text { Coefficient for gas phase viscosity } \\ \mathrm{Cp}_{\mathrm{R}}, \mathrm{k}_{\mathrm{Rcond}} & \text { Heat capacity, thermal conductivity of overburn/underburden } \\ \hat{\mathrm{C}}_{\mathrm{gi}} & \text { The } \mathrm{i}^{\text {th }} \text { heat capacity } \\ \overrightarrow{\mathrm{f}} & \text { Volumetric flow rate } \\ \text { geofac, } \mathrm{k}_{\mathrm{xx}, \mathrm{yy}, z z} & \begin{array}{l}\text { Geology factor of the well, element of permeability tensor in different } \\ \text { direction }\end{array} \\ \mathrm{h}, \mathrm{k}_{\perp}, \mathrm{f}_{\mathrm{h}}, \mathrm{f}, \mathrm{r}_{\mathrm{e}}, \mathrm{r}_{\mathrm{w}}, \mathrm{S} & \begin{array}{l}\text { Legth of well, permeability perpendicular to well, interval length factor, } \\ \text { well fraction, effective radius, well radius, skin factor }\end{array} \\ \widehat{\mathrm{H}}, \widehat{\mathrm{U}}, \overline{\mathrm{U}} & \text { Molar enthalpy, molar internal energy, volumetric internal energy } \\ \mathrm{H}_{\text {evp }}, \mathrm{Ev} & \text { Coefficient for computing heat of evaporation } \\ \mathrm{kr}, \mathrm{kr}_{\mathrm{ocw}}, \mathrm{kr}_{\mathrm{wc}} & \text { Relative permeability, oil relative permeability at ocnnate water, connate } \\ \mathrm{kv}_{\mathrm{i}} & \text { water relative permeability }\end{array}$




$\begin{array}{ll}\mathrm{K}_{\mathrm{i}, \mathrm{p} 1-\mathrm{p} 2} & \text { K-value of component I respect to phase } \mathrm{p} 1 \text { and } \mathrm{p} 2 \\ \mathrm{M} & \text { Molecular weight } \\ \mathrm{Np}, \mathrm{Nc}, \mathrm{Ne}, \mathrm{Nr} & \text { Number of phase, component, thermal equilibrium, reaction in the system } \\ \mathrm{P}, \mathrm{Pc}, \mathrm{P}_{\mathrm{c}} & \text { Pressure, critical pressure, capillary pressure } \\ \mathrm{PI} & \text { Well index } \\ \mathrm{q}_{\mathrm{i}} & \text { Soruce/sink term of the conservation equation of the component } \mathrm{i} \\ \mathrm{S} & \text { Saturation } \\ \mathrm{T}, \mathrm{Tc} & \text { Temperature, critical temperature } \\ \mathrm{V} & \text { Variable } \\ \mathrm{X}_{\mathrm{i}} & \text { Molar fraction of component i in its master phase } \\ \mathrm{x} & \text { Molar fraction } \\ \mathrm{X}_{\mathrm{i}}, \mathrm{y}_{\mathrm{i}} & \text { Molar frction of component i in oil phase, gas phase } \\ \mathrm{z}_{\mathrm{i}} & \text { Global molar fraction of component i } \\ \alpha & \text { Interaction factor in EOS } \\ \phi & \text { Porosity } \\ \gamma & \text { Spacific density } \\ \mu & \text { Viscosity } \\ \Re & \text { Chemical reaction term } \\ \rho & \text { Molar density } \\ \xi & \text { Volumetric mobility }\end{array}$

\section{Chapter Bibliography}

W. L. Buchanan, “Simulating Steam Additive EOR Process,” Soc. Pet. Eng. J., pp. 255-265, 1985.

K.H. Coats, “In-Situ Combustion Model, “Soc. Pet. Eng. J., pp. 533-554, 1980.

Z. Chen, G. Huan, and B. Li, "Modeling 2D and 3D horizontal wells using CVFA," Commun. Math. Sci., vol. 1, pp. 30-44, 2002.

CMG, Stars User's Guide, 2006 ${ }^{\text {th }}$ ed., CMG, 150, 3553-31 Street N.W. Calgary, Alberta, Canada, October 2006.

Eclips Simulaiton Software Manuals, Schlumberger, 2006.

J. W. Grabowski, R. K. Vinsome, R. C. Line, A. Behie, and B. Rubin, "A fully implict general purpose finite-differnece thermal model for in situ combustion and steam,” Soc. Pet. Eng. J., pp.. 1-14, 1979.

B. Rubin and W. L. Buchanan, “A General Purpose Thermal Model,” Soc. Pet. Eng. J., pp. 202216, 1985.

P. K. W. Vinsome and J. Westerveld, "A Simple Method for Predicting Cap and Base Rock Heat Losses in Thermal Reservoir Simulators,” J. Can. Pet. Tech., pp. 6-9, 1980. 


\section{Appendix 7.1}

Table A7.3: Data used in the steamflood example

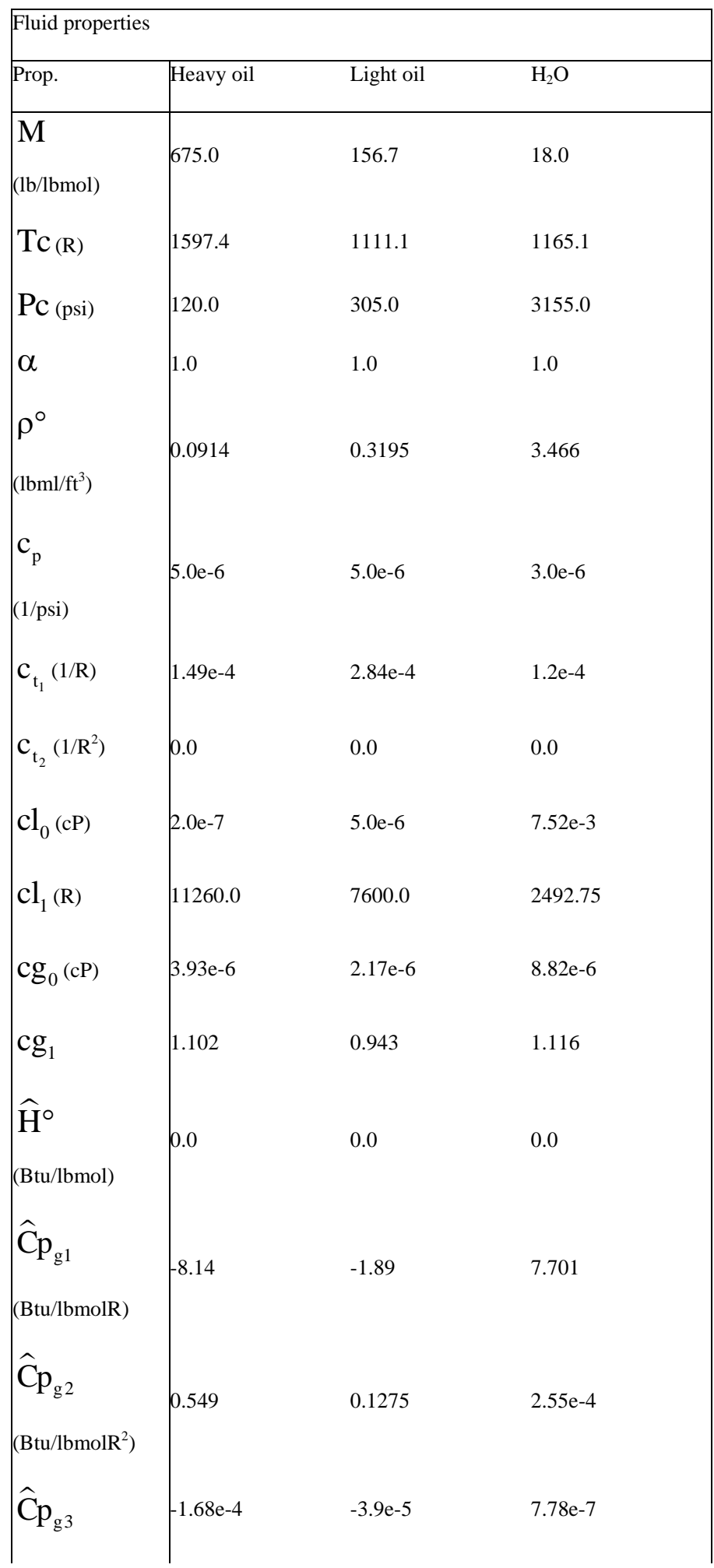




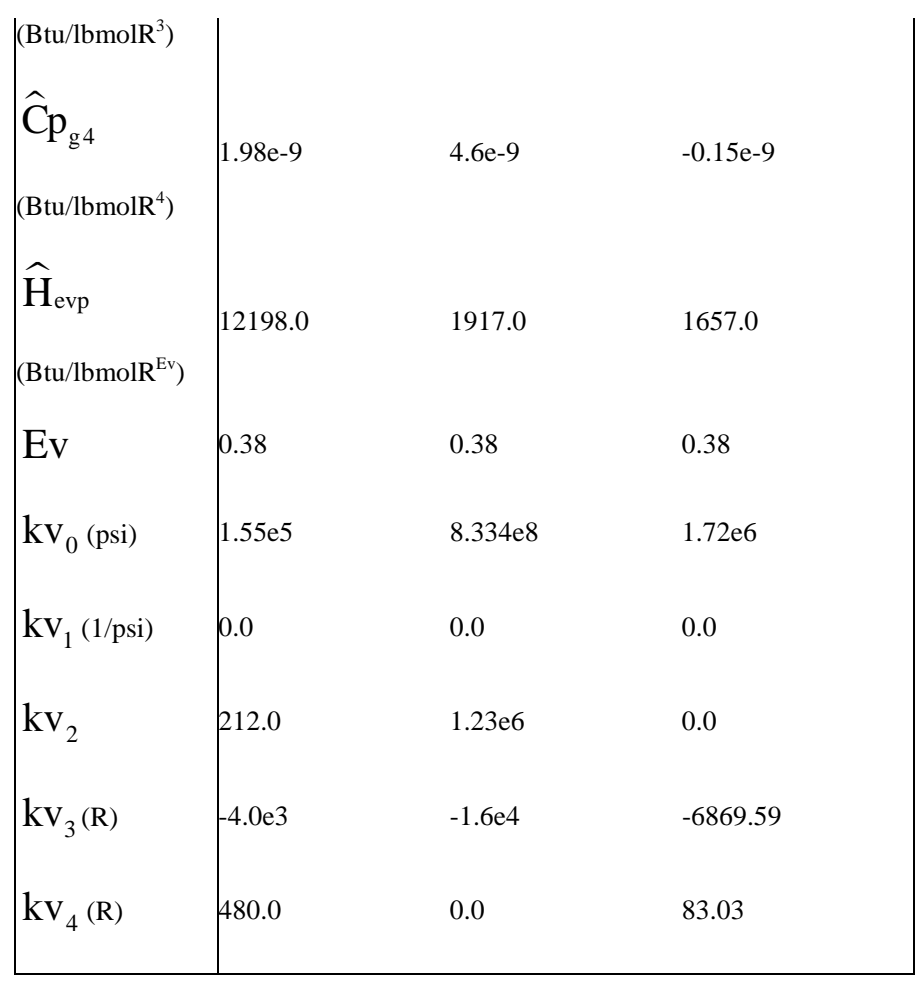

\begin{tabular}{|c|c|c|c|c|}
\hline \multicolumn{5}{|c|}{ Rock properties, matrix } \\
\hline$\phi^{\circ}$ & $\mathrm{Cr}_{\mathrm{p}}(1 / \mathrm{psi})$ & $\begin{array}{l}\overline{\mathrm{U}}_{\mathrm{R}}^{\circ} \\
\left(\mathrm{Btu} / \mathrm{ft}^{3}\right)\end{array}$ & $\begin{array}{l}\mathrm{K}_{\text {abs,matrix }} \\
(\mathrm{mD})\end{array}$ & $\begin{array}{l}\mathrm{k}_{\text {cond,matrix }} \\
\text { (Btu/ftRD) }\end{array}$ \\
\hline 0.25 & 1.e-6 & 35.0 & 1.e2 & 35.0 \\
\hline
\end{tabular}

\begin{tabular}{|lllll|}
\hline \multicolumn{2}{|l|}{ Rock properties, fracture } & & \\
\hline$\phi^{\circ}$ & $\overline{\mathrm{Cr}}_{\mathrm{p}}(1 / \mathrm{psi})$ & $\mathrm{k}_{\mathrm{R}}{ }_{\mathrm{R} s, \text { fractire }}$ & $\mathrm{k}_{\text {cond,fracture }}$ \\
& & $\left(\mathrm{Btu} / \mathrm{ft}^{3}\right)$ & $(\mathrm{mD})$ & $(\mathrm{Btu} / \mathrm{ftRD})$ \\
\hline 0.25 & $1 . \mathrm{e}-6$ & 35.0 & $(2 . \mathrm{e} 2)^{\mathrm{a}}$ & 30.0 \\
& & & $(1 . \mathrm{e} 4)^{\mathrm{b}}$ & \\
\hline
\end{tabular}

\begin{tabular}{|llll|llll|}
\hline \multicolumn{7}{|l|}{ Rock-Fluid properties } \\
\hline $\mathrm{S}_{\mathrm{w}}$ & $\mathrm{kr}_{\mathrm{w}}$ & $\mathrm{kr}_{\mathrm{ow}}$ & $\mathrm{P}_{\text {cow }}$ & $\mathrm{S}_{\mathrm{l}}$ & $\mathrm{kr}_{\mathrm{g}}$ & $\mathrm{kr}_{\mathrm{og}}$ & $\mathrm{P}_{\mathrm{cog}}$ \\
& & & $(\mathrm{psi})$ & & & & $(\mathrm{psi})$ \\
\hline 0.218 & 0.000 & 0.545 & -9.876 & 0.607 & 0.025 & 0.000 & 253.382 \\
0.225 & 0.000 & 0.505 & -9.782 & 0.940 & 0.002 & 0.000 & 39.071
\end{tabular}




\begin{tabular}{|c|c|c|c|c|c|c|c|}
\hline 0.236 & 0.000 & 0.465 & -9.644 & 0.943 & 0.002 & 0.000 & 37.116 \\
\hline 0.247 & 0.001 & 0.427 & -9.506 & 0.946 & 0.002 & 0.001 & 35.159 \\
\hline 0.258 & 0.001 & 0.389 & -9.371 & 0.949 & 0.002 & 0.003 & 33.203 \\
\hline 0.268 & 0.002 & 0.353 & -9.233 & 0.955 & 0.001 & 0.012 & 29.302 \\
\hline 0.278 & 0.003 & 0.318 & -9.107 & 0.961 & 0.001 & 0.031 & 25.399 \\
\hline 0.289 & 0.004 & 0.285 & -8.969 & 0.964 & 0.001 & 0.046 & 23.444 \\
\hline 0.300 & 0.005 & 0.252 & -8.831 & 0.967 & 0.001 & 0.064 & 21.487 \\
\hline 0.311 & 0.006 & 0.221 & -8.697 & 0.970 & 0.001 & 0.088 & 19.530 \\
\hline 0.333 & 0.009 & 0.164 & -8.422 & 0.973 & 0.000 & 0.116 & 17.584 \\
\hline 0.354 & 0.012 & 0.112 & -8.157 & 0.976 & 0.000 & 0.149 & 15.629 \\
\hline 0.364 & 0.014 & 0.089 & -8.022 & 0.979 & 0.000 & 0.183 & 13.672 \\
\hline 0.375 & 0.016 & 0.067 & -7.884 & 0.982 & 0.000 & 0.220 & 11.716 \\
\hline 0.386 & 0.018 & 0.048 & -7.748 & 0.985 & 0.000 & 0.265 & 9.771 \\
\hline 0.397 & 0.020 & 0.031 & -7.612 & 0.988 & 0.000 & 0.311 & 7.815 \\
\hline 0.407 & 0.022 & 0.017 & -7.483 & 0.991 & 0.000 & 0.362 & 5.858 \\
\hline 0.418 & 0.025 & 0.006 & -7.348 & 0.994 & 0.000 & 0.419 & 3.901 \\
\hline 0.429 & 0.027 & 0.000 & -7.210 & 0.997 & 0.000 & 0.480 & 1.957 \\
\hline 1.000 & 0.292 & 0.000 & 0.000 & 1.000 & 0.000 & 0.545 & 0.000 \\
\hline
\end{tabular}

\begin{tabular}{|l|l|}
\hline \multicolumn{2}{|l|}{ Initial conditions } \\
\hline $\mathrm{P}(\mathrm{psi})$ & 250.0 \\
$\mathrm{~T}(\mathrm{R})$ & 0.06 \\
$\mathrm{Z}_{\mathrm{HO}}$ & 0.04 \\
$\mathrm{Z}_{\mathrm{LO}}$ & 0.90 \\
$\mathrm{Z}_{\mathrm{H}_{2} \mathrm{O}}$ & \\
\hline
\end{tabular}

\begin{tabular}{|l|ll|}
\hline \multicolumn{2}{|c|}{ Well conditions } \\
\hline & Producer & Injecitor \\
\hline P (psi) & 100.0 & 350.0 \\
T (R) & & 892.0 \\
Steam Qaul. & & $70.0 \%$ \\
\hline
\end{tabular}


Table 8.4: Component-phase partitioning in in-situ combustion

\begin{tabular}{|l|l|llll|}
\hline Component ID & Type & Oil & Gas & Water & Solid \\
\hline Heavy oil & Oleic & $\checkmark$ & $\checkmark$ & & \\
Light oil & Oleic & $\checkmark$ & $\checkmark$ & & \\
$\mathrm{CO}_{2}+\mathrm{N}_{2}$ & Oleic & $\checkmark$ & $\checkmark$ & & \\
$\mathrm{O}_{2}$ & Oleic & $\checkmark$ & $\checkmark$ & & \\
$\mathrm{H}_{2} \mathrm{O}$ & Aqueous & & $\checkmark$ & $\checkmark$ & \\
Coke & Solid & & & & $\checkmark$ \\
\hline
\end{tabular}

Table 8.5: Property data in steaming flooding

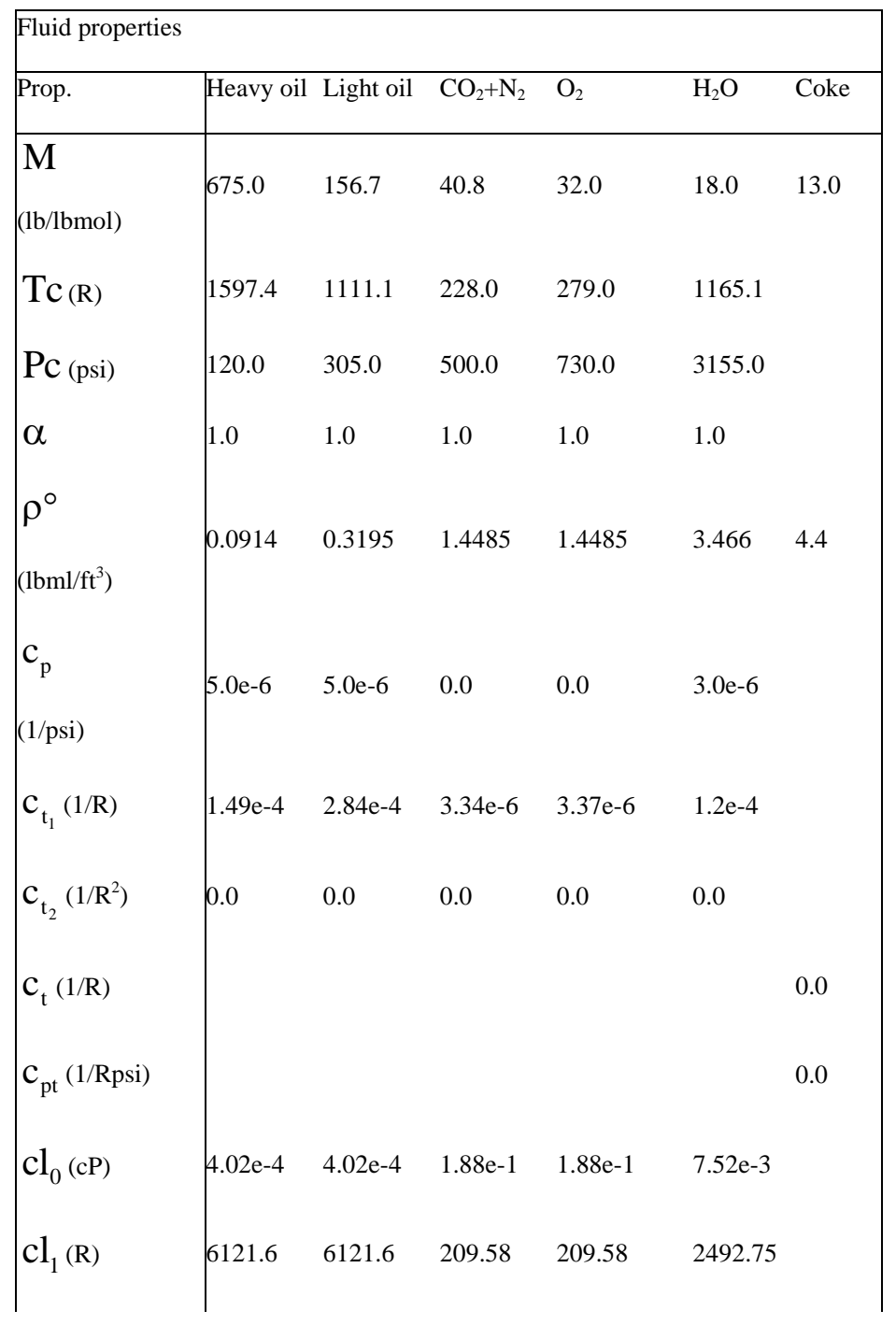




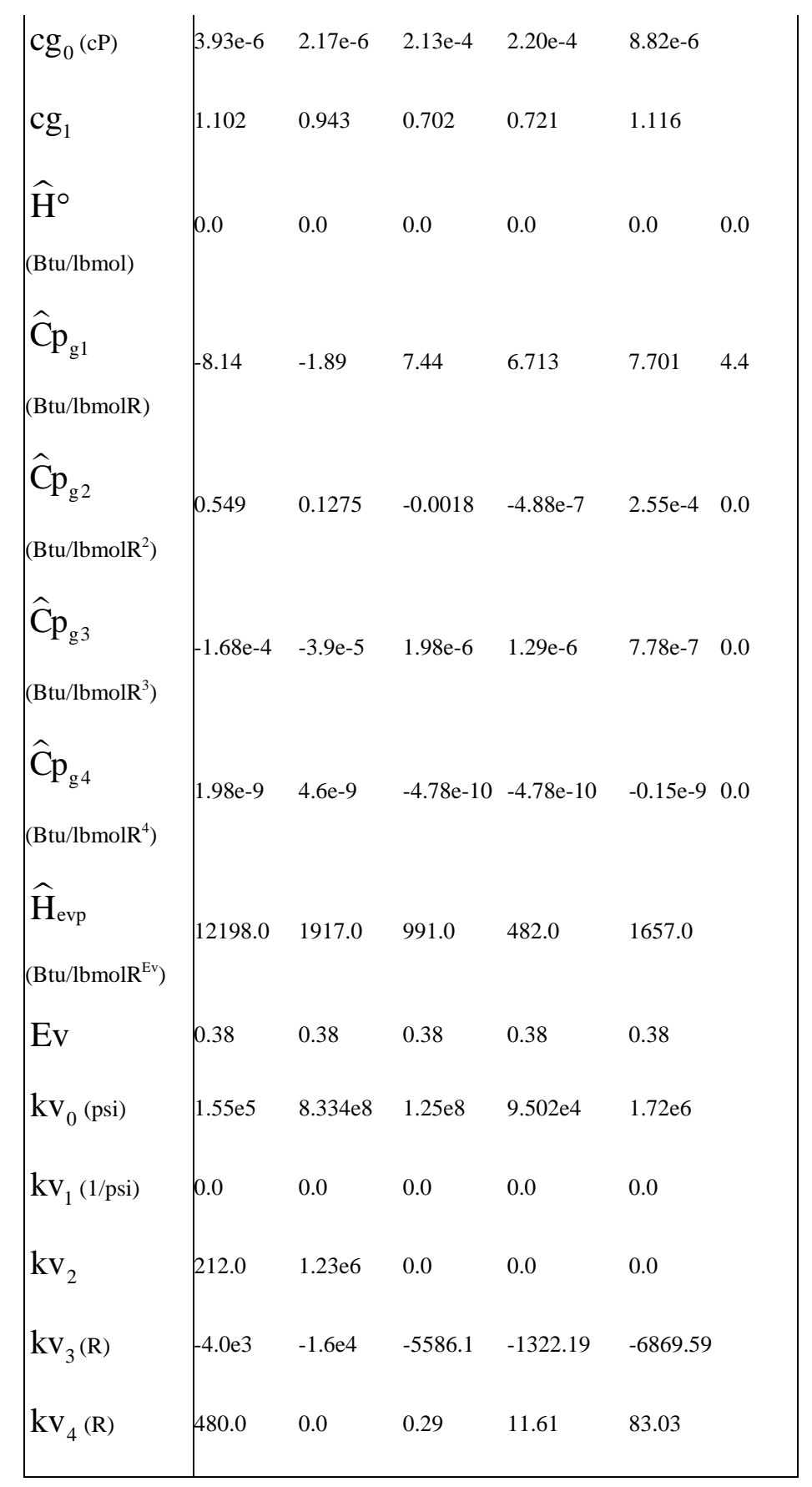

\begin{tabular}{|l|}
\hline Chemical reacitons \\
\hline $\mathrm{HO} \rightarrow 2.154 \mathrm{LO}+25.96$ Coke \\
rate $=\mathrm{kC}_{\mathrm{HO}}$ \\
Ea $($ Btu/lbmolR $)=27000.0 ; \mathrm{k}_{0}(1 / \mathrm{D})=1 . \mathrm{e} 7$ \\
\hline
\end{tabular}




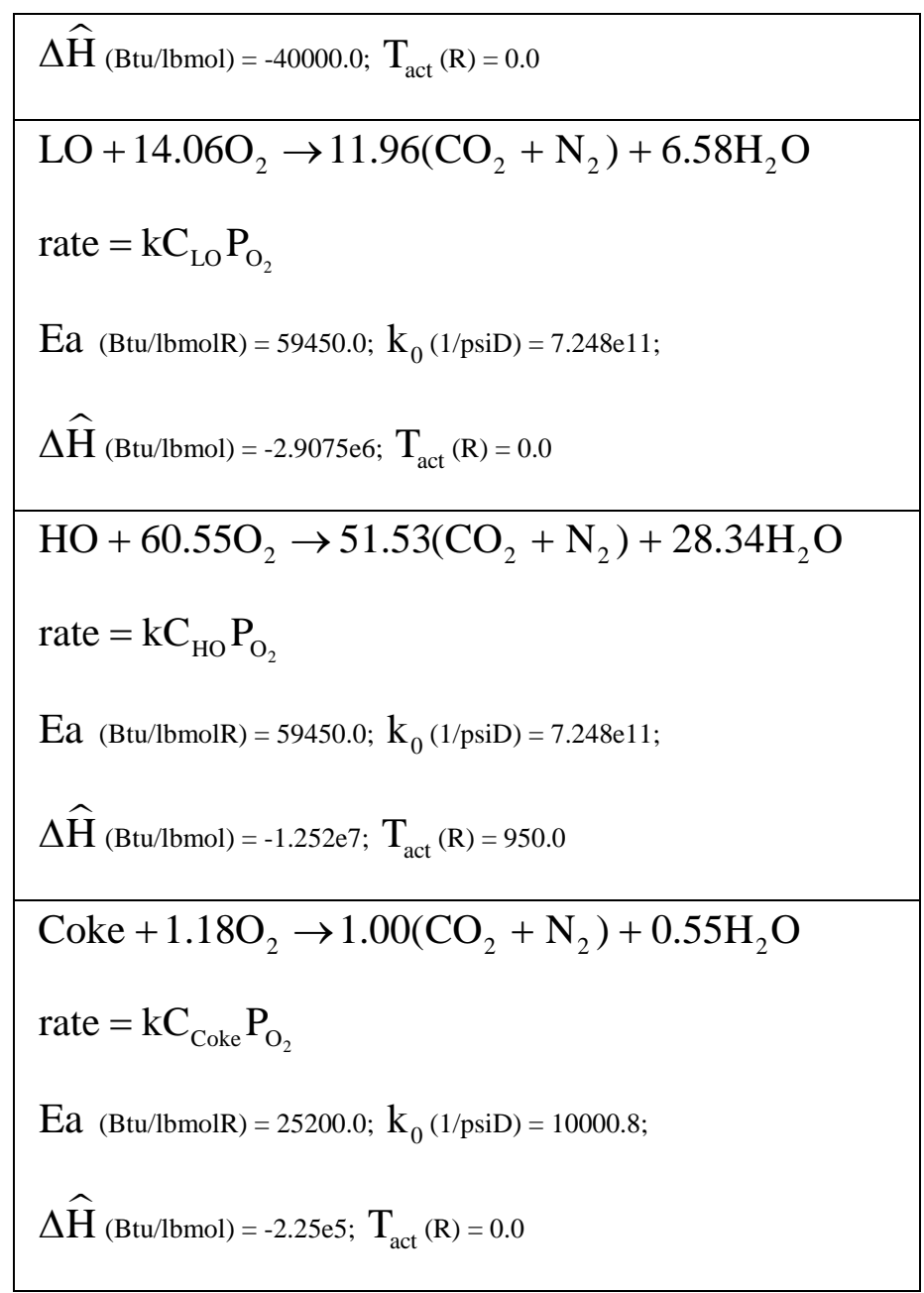

\begin{tabular}{|lllll|}
\hline Rock properties & & & & \\
\hline$\phi^{\circ}$ & $\mathrm{Cr}_{\mathrm{p}}(1 / \mathrm{psi})$ & $\overline{\mathrm{U}}^{\circ}{ }_{\mathrm{R}}$ & $\mathrm{k}_{\mathrm{abs}}$ & $\mathrm{k}_{\text {cond }}$ \\
& & $\left(\mathrm{Btu} / \mathrm{ft}^{3}\right)$ & $(\mathrm{mD})$ & $(\mathrm{Btu} / \mathrm{ftRD})$ \\
\hline 0.25 & 0.0 & 35.0 & $1 . \mathrm{e} 3$ & 30.0 \\
\hline
\end{tabular}

\begin{tabular}{|ll|}
\hline $\begin{array}{l}\text { Over/under } \\
\text { Burden properties }\end{array}$ \\
\hline $\mathrm{Cp}_{\mathrm{R}}\left(\mathrm{Btu} / \mathrm{ft}^{3} \mathrm{R}\right)$ & $\begin{array}{l}\mathrm{k}_{\mathrm{Rcond}} \\
(\mathrm{Btu} / \mathrm{ftRD})\end{array}$ \\
\hline 35.0 & 24.0 \\
\hline
\end{tabular}




\begin{tabular}{|llll|llll|}
\hline \multicolumn{7}{|l|}{ Rock-Fluid properties } \\
\hline $\mathrm{S}_{\mathrm{w}}$ & $\mathrm{kr}_{\mathrm{w}}$ & $\mathrm{kr}_{\mathrm{ow}}$ & $\mathrm{P}_{\text {cow }}$ & $\mathrm{S}_{\mathrm{l}}$ & $\mathrm{kr}_{\mathrm{g}}$ & $\mathrm{kr}_{\mathrm{og}}$ & $\mathrm{P}_{\text {cog }}$ \\
& & & $(\mathrm{psi})$ & & & & $(\mathrm{psi})$ \\
\hline 0.100 & 0.00 & 0.900 & 0.0 & 0.210 & 0.784 & 0.000 & 0.0 \\
0.2500 & 0.004 & .0600 & 0.0 & 0.320 & 0.448 & 0.010 & 0.0 \\
0.440 & 0.024 & 0.280 & 0.0 \\
0.560 & 0.072 & 0.144 & 0.0 & 0.400 & 0.288 & 0.024 & 0.0 \\
0.672 & 0.168 & 0.048 & 0.0 & 0.472 & 0.184 & 0.052 & 0.0 \\
0.752 & 0.256 & 0.000 & 0.0 & 0.580 & 0.086 & 0.152 & 0.0 \\
1.000 & 1.000 & 0.000 & 0.0 & 0.680 & 0.024 & 0.272 & 0.0 \\
& & & & 0.832 & 0.006 & 0.448 & 0.0 \\
& & & & 0.872 & 0.000 & 0.900 & 0.0 \\
& & & & 0.000 & 0.000 & 0.900 & 0.0 \\
\hline
\end{tabular}

\begin{tabular}{|c|c|}
\hline \multicolumn{2}{|c|}{ Initial conditions } \\
\hline $\mathrm{P}_{(\mathrm{psi})}$ & 2000.0 \\
\hline $\mathrm{T}(\mathrm{R})$ & $610.0(850.0)^{\mathrm{a}}$ \\
\hline $\mathrm{z}_{\mathrm{HO}}$ & 0.085 \\
\hline $\mathrm{Z}_{\mathrm{LO}}$ & 0.005 \\
\hline $\mathrm{Z}_{\mathrm{CO}_{2}+\mathrm{N}_{2}}$ & 0.0059 \\
\hline $\mathrm{Z}_{\mathrm{O}_{2}}$ & 0.004 \\
\hline $\mathrm{Z}_{\mathrm{H}_{2} \mathrm{O}}$ & 0.9 \\
\hline $\mathrm{Z}_{\text {Coke }}$ & 0.0001 \\
\hline
\end{tabular}

\begin{tabular}{|l|l|l|l|}
\hline \multicolumn{2}{|c|}{ Well conditions } \\
\hline \multirow{2}{*}{} & \multirow{2}{*}{ Producer } & \multicolumn{2}{|l|}{ Injector } \\
\cline { 3 - 4 } & & Dry & Wet $^{\mathrm{b}}$ \\
\hline & & & \\
\hline $\mathrm{P}(\mathrm{psi})$ & 1980.0 & 2200.0 & 2200.0 \\
$\mathrm{~T}(\mathrm{R})$ & & 650.0 & 650.0 \\
\hline
\end{tabular}




\begin{tabular}{|c|c|c|c|c|c|}
\hline $\mathrm{X}_{\mathrm{g}, \mathrm{CO}_{2}+\mathrm{N}_{2}}$ & 0.79 & \multicolumn{4}{|l|}{0.786} \\
\hline $\mathrm{x}_{\mathrm{g}, \mathrm{O}_{2}}$ & 0.21 & \multicolumn{4}{|c|}{0.2093} \\
\hline $\mathrm{X}_{\mathrm{g}, \mathrm{H}_{2} \mathrm{O}}$ & & \multicolumn{4}{|c|}{0.0047} \\
\hline Gas & & & & & \\
\hline Water & & 99.0 & 98.0 & 85.0 & 80.0 \\
\hline (vol. ratio) & & 1.0 & 2.0 & 15.0 & 20.0 \\
\hline
\end{tabular}

${ }^{a}$ : ignition zone

${ }^{\mathrm{b}}$ : water co-injeciton begins between $20-25^{\text {th }}$ days

\section{Appendix 7.2}

K-value equation in this thermal model is only a function of temperature and pressure. For a component 'i', the molar fraction in phases ' $\mathrm{p} 1$ ' and ' $\mathrm{p} 2$ ' can be computed by the following equation.

$$
\begin{aligned}
& \mathrm{K}_{\mathrm{i}, \mathrm{p}_{\mathrm{L}}-\mathrm{p}_{\mathrm{H}}}=\frac{\mathrm{X}_{\mathrm{p}_{\mathrm{L}}, \mathrm{i}}}{\mathrm{X}_{\mathrm{p}_{\mathrm{H}}, \mathrm{i}}} \\
& =\left(\frac{\mathrm{kv}_{0}}{\mathrm{P}}+\mathrm{kv}_{1} \mathrm{P}+\mathrm{kv}_{2}\right) \exp \left(\frac{\mathrm{kv}_{3}}{\mathrm{~T}-\mathrm{kv}_{4}}\right)
\end{aligned}
$$

Pure component properties in each phase are computed first. Mixing rules are used to obtain the phase properties. Equations to compute molar density, viscosity and enthalpy for pure component are shown below

\section{Molar density: [lbmol/ft $\left.\mathbf{f}^{3}\right]$}

$$
\begin{aligned}
& \text { liquid: } \rho=\frac{\rho^{\circ}}{\exp \left(c_{t_{1}}\left(T-T_{0}\right)+0.5 c_{t_{2}}\left(T^{2}-T_{0}^{2}\right)-c_{p}\left(P-P_{0}\right)\right)} \text { solid: } \\
& \rho=\frac{\rho^{\circ}}{\exp \left(c_{t_{1}}\left(T-T_{0}\right)-c_{p t}\left(P-P_{0}\right)\left(T-T_{0}\right)\right)}
\end{aligned}
$$




\section{Viscosity: [cP]}

liquid:

$$
\mu=\mathrm{cl}_{0} \exp \left(\frac{\mathrm{cl}_{1}}{\mathrm{~T}}\right)
$$

$\mu_{\mathrm{w}}=\mathrm{Cw}_{0}+\mathrm{cw}_{1} \mathrm{~T}$ (for water)

gas:

$\mu_{\mathrm{g}}=\mathrm{cg}_{0} \mathrm{~T}^{\mathrm{cg}_{1}}$

\section{Enthalpy: [Btu/lbmol]}

liquid:

$$
\widehat{\mathrm{H}}=\widehat{\mathrm{H}}_{\mathrm{IG}}^{\circ}+\int_{\mathrm{T}^{\circ}}^{\mathrm{T}}<\widehat{\mathrm{C}}_{\mathrm{g}}>\mathrm{dT}-\widehat{\mathrm{H}}_{\text {evp }}(\mathrm{Tc}-\mathrm{T})^{\mathrm{Ev}}
$$

gas:

$\widehat{\mathrm{H}}=\widehat{\mathrm{H}}_{\mathrm{IG}}^{\circ}+\int_{\mathrm{T}^{\circ}}^{\mathrm{T}}<\widehat{\mathrm{C}}_{\mathrm{g}}>\mathrm{dT}$

solid:

$\widehat{\mathrm{H}}=\widehat{\mathrm{H}}_{\mathrm{s}}^{\circ}+\int_{\mathrm{T}^{\circ}}^{\mathrm{T}}<\widehat{\mathrm{C}}_{\mathrm{s}}>\mathrm{dT}$

where

$<\hat{\mathrm{C}} \mathrm{p}_{\mathrm{p}}>=\hat{\mathrm{C}} \mathrm{p}_{\mathrm{p} 0}+\hat{\mathrm{C}} \mathrm{p}_{\mathrm{p} 1} \mathrm{~T}+\hat{\mathrm{C}} \mathrm{p}_{\mathrm{p} 2} \mathrm{~T}^{2}+\hat{\mathrm{C}} \mathrm{p}_{\mathrm{p} 3} \mathrm{~T}^{3}+\ldots$

Phase properties such as molecular weight, molar density, pecific mass density, viscosity, enthalpy and internal energy are computed by using the following equations.

Molecular weight: [lb/lbmol] 
$\mathrm{M}_{\mathrm{p}}=\sum_{\mathrm{i}=1}^{\mathrm{Nc}} \mathrm{x}_{\mathrm{p}, \mathrm{i}} \mathrm{M}_{\mathrm{i}}$

Molar density:[lbmol / $\left.\mathrm{ft}^{3}\right]$

liquid:

$\frac{1}{\rho_{1}}=\sum_{\mathrm{i}=1}^{\mathrm{Nc}} \frac{\mathrm{x}_{\mathrm{l}, \mathrm{i}}}{\rho_{\mathrm{l}, \mathrm{i}}}$

gas (Redlich KWong EOS):

$$
\begin{aligned}
& \rho_{g}=\frac{P}{Z_{g} R_{g} T} \\
& Z_{g}^{3}-Z_{g}^{2}+\left(A-B-B^{2}\right) Z_{g}-A B=0 \\
& A=0.42747 \frac{P}{T^{2}} \sum_{i=1}^{N c}\left(x_{g, i} \frac{T_{i}^{2} \alpha_{i}^{0.5}}{P c_{i}^{0.5}}\right)^{2} \\
& A=0.08664 \frac{P}{T} \sum_{i=1}^{N c}\left(x_{g, i} \frac{T_{i}}{P c_{i}}\right)
\end{aligned}
$$

solid: the same as liquid

Specific mass density: $\left[\mathrm{lb}_{\mathrm{f}} / \mathrm{ft}^{3}\right]$

$\gamma_{p}=\rho_{p} M_{p} \frac{g}{g_{c}}$

Viscosity: [cP]

liquid: 
$\ln \mu_{1}=\sum_{\mathrm{i}=1}^{\mathrm{Nc}} \mathrm{x}_{\mathrm{l}, \mathrm{i}} \ln \mu_{\mathrm{l}, \mathrm{i}}$

gas:

$\mu_{\mathrm{g}}=\frac{\sum_{\mathrm{i}=1}^{\mathrm{Nc}} \mathrm{x}_{\mathrm{p}, \mathrm{i}} \sqrt{\mathrm{M}_{\mathrm{i}}} \mu_{\mathrm{g}, \mathrm{i}}}{\sum_{\mathrm{i}=1}^{\mathrm{Nc}} \mathrm{x}_{\mathrm{p}, \mathrm{i}} \sqrt{\mathrm{M}_{\mathrm{i}}}}$

\section{Enthalpy: [Btu / lbmol]}

$$
\widehat{\mathrm{H}}_{\mathrm{p}}=\sum_{\mathrm{i}=1}^{\mathrm{Nc}} \mathrm{x}_{\mathrm{p}, \mathrm{i}} \widehat{\mathrm{H}}_{\mathrm{p}, \mathrm{i}}
$$

\section{Internal energy: [Btu / lbmol]}

$$
\widehat{\mathrm{U}}_{\mathrm{p}}=\widehat{\mathrm{H}}_{\mathrm{p}}-\frac{\mathrm{P}}{\rho_{\mathrm{p}}}
$$

Rock (inert component of the reservoir) properties are computed as follow,

Internal energy: [Btu / $\left.\mathbf{f t}^{3}\right]$

$\overline{\mathrm{U}}_{\mathrm{R}}=\overline{\mathrm{U}}_{\mathrm{R}}^{\circ}+\left(\mathrm{cr}_{\mathrm{t}_{0}}+\mathrm{cr}_{\mathrm{t}_{1}} \mathrm{~T}\right)\left(\mathrm{T}-\mathrm{T}_{\mathrm{i}}\right)$

Porosity:

$\phi=\phi^{\circ}\left(1+\mathrm{cr}_{\mathrm{p}}\left(\mathrm{P}-\mathrm{P}_{0}\right)\right)$

Rock-Fluid interaction properties such as capillary pressures and relative permeabilities are computed by RockFluid module. For a three-phase system (oil, water and gas), we have two 
capillary pressures: $\mathrm{Pc}_{\mathrm{og}}$ and $\mathrm{Pc}_{\mathrm{ow}}$. In general, $\mathrm{Pc}_{\mathrm{og}}$ is a function of $\mathrm{S}_{\mathrm{g}}$ and $\mathrm{Pc}_{\mathrm{ow}}$ is a function of $\mathrm{S}_{\mathrm{w}}$.

The phase pressure therefore is computed by

$$
\mathrm{P}_{\mathrm{P}}=\mathrm{P}_{0}+\mathrm{P}_{\text {cop }}
$$

The derivative of phase pressure is computed as

$$
\frac{\partial \mathrm{P}_{\mathrm{p}}}{\partial \mathrm{v}}=\frac{\partial \mathrm{P}_{0}}{\partial \mathrm{v}}+\sum_{\mathrm{p}=1}^{\mathrm{Np}} \frac{\partial \mathrm{P}_{\text {cop }}}{\partial \mathrm{S}_{\mathrm{p}}} \frac{\partial \mathrm{S}_{\mathrm{p}}}{\partial \mathrm{v}}
$$

Stone II model is used [8] for relative permeability calculation. Look-up tables are used to obtain $\mathrm{kr}_{\mathrm{w}}$ and $\mathrm{kr}_{\mathrm{g}}$, which are functions of $\mathrm{S}_{\mathrm{w}}$ and $\mathrm{S}_{\mathrm{g}}$, respectively. Relative permeability of the oil phase is computed as

$$
\mathrm{kr}_{\mathrm{o}}=\mathrm{kr}_{\mathrm{ocw}}\left\{\left(\frac{\mathrm{kr}_{\mathrm{ow}}}{\mathrm{kr}_{\mathrm{ocw}}}+\mathrm{kr}_{\mathrm{w}}\right)\left(\frac{\mathrm{kr}_{\mathrm{og}}}{\mathrm{kr}_{\mathrm{ocw}}}+\mathrm{kr}_{\mathrm{g}}\right)-\mathrm{kr}_{\mathrm{w}}-\mathrm{kr}_{\mathrm{g}}\right\}
$$

Power law rate expression is used to account for the generation and consumption of the components from chemical reactions. Stoichiometries of chemical reaction and rate expressions for each reaction are defined in the input file. In the application, the rate expression for any reaction should be simplified into the form

$$
\Re_{\mathrm{r}}=\mathrm{k}_{0} \exp \left(\frac{\mathrm{Ea}}{\mathrm{R}_{\mathrm{h}} \mathrm{T}}\right) \prod_{\mathrm{i}=1}^{\mathrm{Nc}} \mathrm{C}_{\mathrm{i}}^{\mathrm{ni}}
$$

Reaction rate for each reaction will be pre-factored by stoichiometry of the specified component and added to component's conservation equation. 


\section{Chapter 8. Parallel Computation}

\section{Table of Contents}

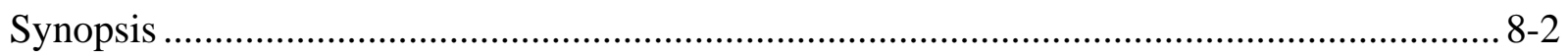

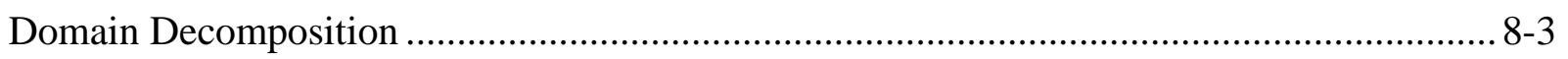

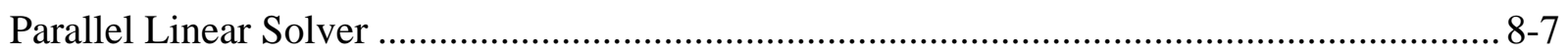

Parallel Equation Decoupling ............................................................................................. 8-8

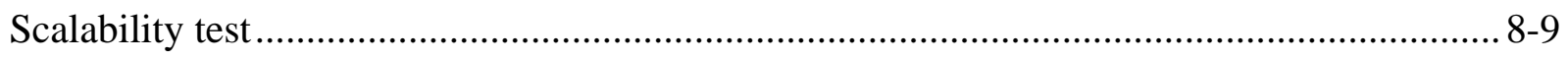

Parallel Performance .......................................................................................................... 8-10

Chapter Bibliography .................................................................................................... 8-11 


\section{Synopsis}

In general, the high level of computational effort in reservoir simulations is required because of two considerations; the necessity of high resolution simulations due to the explicit description of detailed geological features and requirement of solving complex coupled relationships (for example, geomechanical coupling with the multiphase flow problem, and the handling of numerous components). In addition, computational requirements increase as the scale of the problem increases, and the number of control-volumes or grid blocks increase. One possible solution is parallel computing to perform the simulation on a number of processors at the same time and acquire results in a reasonable period of time. Thus, parallel computation capability becomes a necessary part of the modern reservoir simulator.

The parallel computing capability is provided at the framework level, which ensures that the parallel computation capability is available no matter which DM and PM are combined later at compilation time. Currently the parallel computing routine exists as a semi-independent submodule in PM (it is physically implemented in PM but it is independent of the implementation of any PM module. Another efficient option would be to provide parallel computing as an independent utility.

In this chapter, we introduce the approach being adopted in our framework to achieve parallel computing of the reservoir simulation by describing the following components: domain decomposition, parallel linear solver and parallel Gaussian elimination. 


\section{Domain Decomposition}

Domain decomposition is the first step to achieve parallelization. In domain decomposition, the original domain (including fractures) is divided into number of subdomains, each of which is distributed to one processor. All processors then execute parallel computations on their associated subdomains. Message transfers between processors are required to obtain the crossboundary information. Domain decomposition in our research is achieved using METIS, which is a family of programs for partitioning unstructured graphs and hypergraphs and computing fillreducing orderings of sparse matrices. The underlying algorithms used in METIS are based on the state-of-the-art multilevel paradigm that has been shown to produce high quality results. This means that the approach can be used for very large problems with good scalability (Karypis and Kumar, 2004). ParMETIS is an MPI-based implementation and it extends the functionality provided by METIS and includes routines that are especially designed for parallel AMR (adaptive mesh refinement) computations and large scale numerical simulations (Karypis et al, 2006).

In ParMETIS, the k-way partitioning of a graph on $\mathrm{P}$ processors using the multilevel k-way multi-constraint partitioning algorithm is performed by calling a message-passing interface (MPI) routine. The detailed descriptions of the functions and the usage of the arguments in the routine can be found in their manuals (Karypis et al, 2006)

To use METIS/ParMETIS effectively, a graph describing how the nodes (in our applications, nodes are the control volumes and the segments of wells ) are connected is required. A weighting value can be assigned to each node to control the load balance of the partition results. In discrete fracture model, no special treatment for fractures is required, if the fracture graph is 
provided. Weighting values are set to unity for most applications which means the partitioning is based purely on the connection relationships. A standalone METIS/ParMETIS code was written, and is used prior to the simulation, to decompose the domain and to generate the subdomain segments later used as input data for parallel computing.

Each sub-domain is divided into two components:

- Host finite volumes: A component consisting of finite volumes residing in that subdomain.

- Ghost finite volume: They are at the boundary of the opposite sub-domain. They provide the information required in flow computation.

Two lists are required to complete the local linear system for each process, a list of finite volumes including the local host finite volumes and the ghost finite volumes, and a map of the all the connections including the connections between the host finite volumes (intra-process connections) and host to ghost finite volumes (inter-process connections). The residual function and the derivatives of the accumulation term and chemical reaction term in the conservation equation are computed for those host finite volumes in the list and constraint equations are computed for all the finite volumes. This is done to facilitate the Gaussian elimination in parallel. The computation of the flow terms are completed by going through the connectivity list mentioned above.

During domain decomposition, placing a well into more than one subdomain should be avoided. From a load balancing point of view, partitioning a well into different sub-domains is oftentimes unavoidable. When a well is partitioned, one processor is assigned to be the host of the well 
(usually the finite volume connected to the well head) and all finite volumes connected to that particular well at different processors are collected as ghosts.

The domain decomposition method used in our simulator is shown as an example in Figure 8.1, where the original domain is split into two subdomains. In this example, a well penetrating the boundary of two subdomains is assigned to processor I. The finite volumes connected to the well in domain Proc. II have

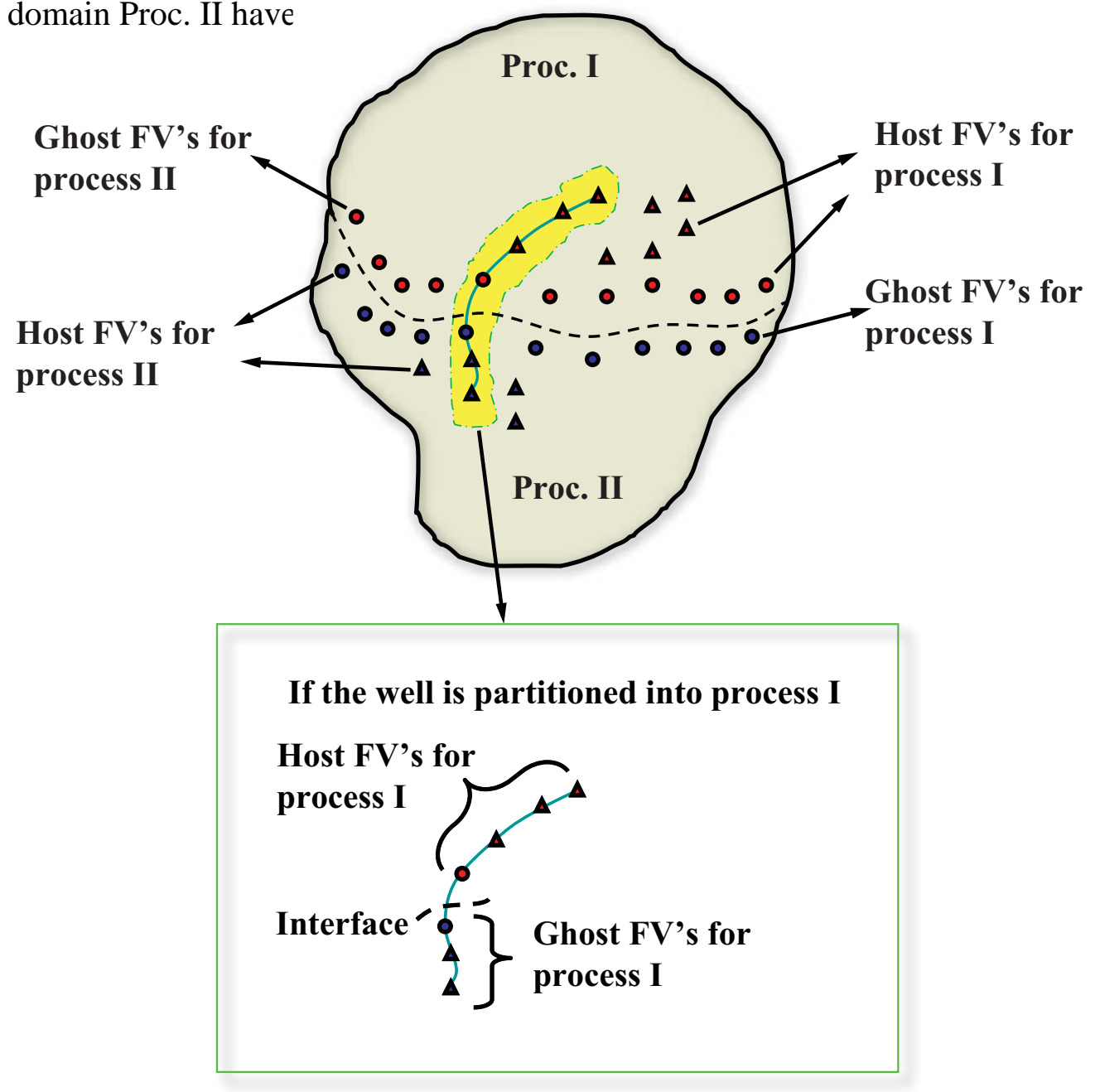

Figure 8.1 The partitioning scheme used in the simulator 
Local linear system is setup first on each processor, and then the global system is assembled through message passing and solved by parallel linear solver. A schematic diagram showing the structure of the global linear system is shown in Figure 8.2. For this particular case, the elements on the main diagonal of the global Jacobian matrix are hosts while those in other positions are ghost elements.
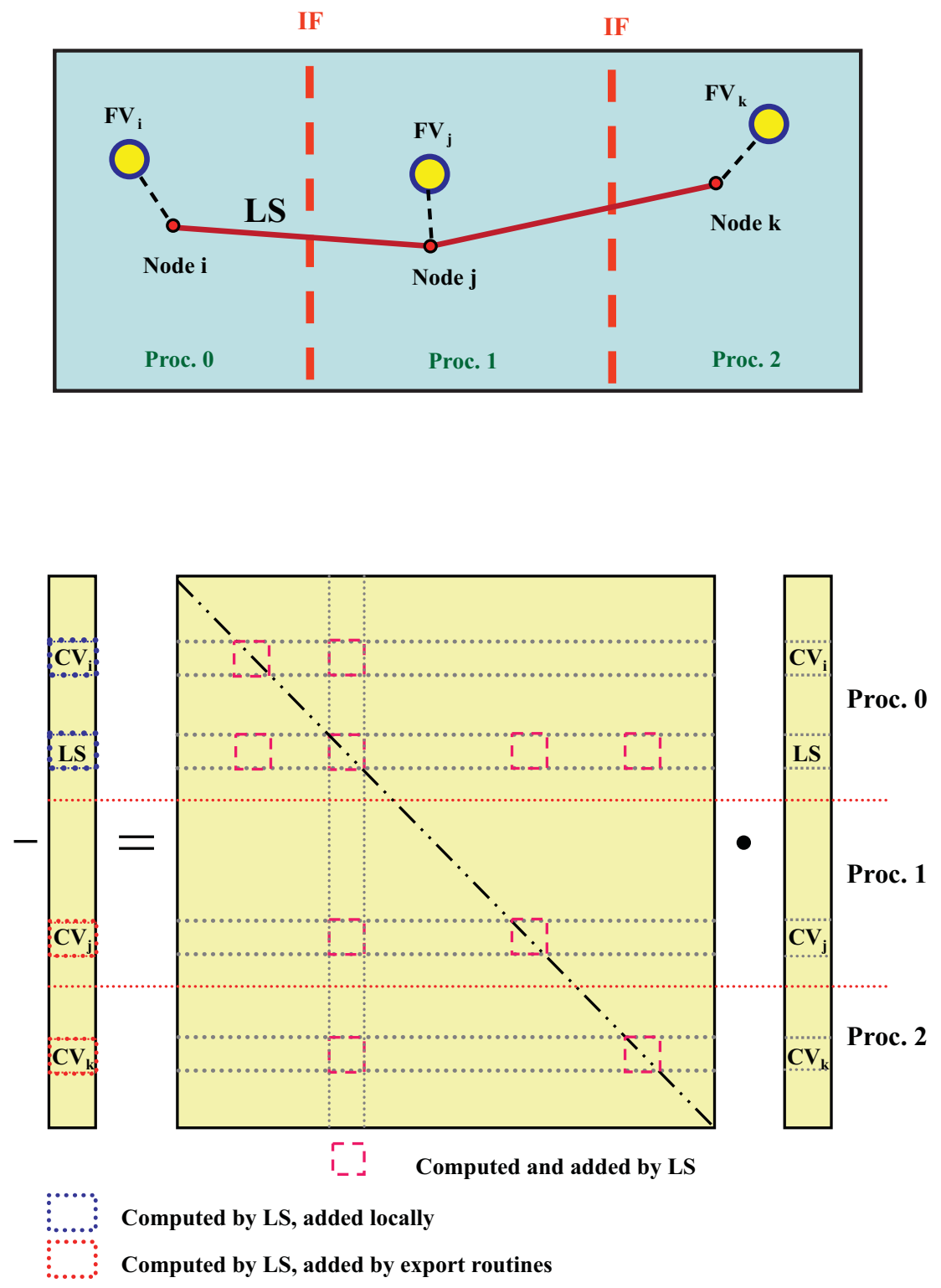

Figure 8.2 The structure of the linear solver after partitioning 
Load balance control is important for increasing the performance of parallel computation. There are many strategies for controlling load balance. In homogeneous parallel machines, this is achieved by distributing even numbers of the finite volumes on each processors which ensure that the linear systems solved on different processors have approximately equal size. However, for heterogeneous machines where processors may have CPUs with different performance indices or clock rates. The load balance in the later case has to take the performance of each processor into account. In this work, we use the default setting in ParMETS for domain decomposition and all other aspects of a comprehensive load balancing study will be considered in the future.

\section{Parallel Linear Solver}

There are many numerical packages, either open source or commercial, providing parallel linear solver for use. In our framework, the parallel linear solvers are utilized from external libraries and a universal interface is written between the simulator and the routines required. For most of these packages, a map or a graph which gives the details of the connections of the elements in the linear system is first required in order to assemble the local linear system and data exchange during the solution of the global system. The second step is usually to provide the parameters for setting up and optimizing the linear solver. These include the selection of the algorithm of the linear solver and pre-conditioner to be used. Sometimes the performance of the solver can be optimized by pre-allocating the memory. Usually, after these two steps the linear solver is ready to be used. Some solvers do need preprocessing on the input data or postprocessing on the output data to be able to function correctly with the simulator. Currently in the framework we have implemented PETSc (Balay et al, 2007) and Trilinos (Heroux and Willenbring, 2003), two 
numerical packages for providing linear solver used in both the serial and parallel mode. The selection of the linear solver is specified in the input file.

\section{Parallel Equation Decoupling}

Equation decoupling technique can also be used to reduce the size of the linear system to be solved on each processor in parallel computation. In this section, a procedure for decoupling the equations by using Gaussian elimination procedure is discussed. This does not need of interprocess communications.

In parallel model, the local linear system is composed by:

$$
\begin{aligned}
& -\left(\left\{\begin{array}{c}
R_{\mathrm{P} I} \\
R_{\mathrm{s} I}
\end{array}\right\}_{\sum H \text { ost }}+\left\{\begin{array}{c}
R_{\mathrm{P} I} \\
0
\end{array}\right\}_{\sum \text { Ghost }}\right) \\
& =\left[\begin{array}{cc}
A_{I} & B_{I} \\
C_{I} & D_{I}
\end{array}\right]_{\sum H \text { ost }} \cdot\left\{\begin{array}{c}
\delta Y_{\mathrm{P} I} \\
\delta Y_{\mathrm{s} I}
\end{array}\right\}_{\sum H \text { ost }}+\left[\begin{array}{cc}
A_{I} & B_{I} \\
0 & 0
\end{array}\right]_{\sum \text { Ghost }} \cdot\left\{\begin{array}{c}
\delta Y_{\mathrm{P} I} \\
\delta Y_{\mathrm{s} I}
\end{array}\right\}_{\sum \text { Ghost }} \\
& +\sum_{J=1}^{N_{J}}\left[\begin{array}{cc}
A_{I, J} & B_{I, J} \\
0 & 0
\end{array}\right]_{\sum H \text { Host }} \cdot\left\{\begin{array}{l}
\delta Y_{\mathrm{P} J} \\
\delta Y_{\mathrm{s} J}
\end{array}\right\}_{\sum H \text { ost }} \\
& +\sum_{J=1}^{N_{J}}\left[\begin{array}{cc}
A_{I, J} & B_{I, J} \\
0 & 0
\end{array}\right]_{\sum \text { Ghost }} \cdot\left\{\begin{array}{l}
\delta Y_{\mathrm{P} J} \\
\delta Y_{\mathrm{s} J}
\end{array}\right\}_{\sum \text { Ghost }}
\end{aligned}
$$

where the subscripts $\sum$ Host denote that the properties are obtained from the information provided from the host finite volumes and $\sum$ Ghost denotes that the information from ghost finite volumes are required. 
In the intra-process flow computation, every connection is computed only once. However, in the inter-processor flow computation, the same flow connection is computed twice: once from the opposite side. It can be easily seen that all the components in the residual functions and the Jacobian matrix shown in the above equation can be obtained intra-process without communications between processors. However, to eliminate $A_{I, J}$ and $B_{I, J}$ which are the derivatives for the flow between host and ghost finite volumes, we need components $C_{I} \mathrm{~s}$ and $D_{I} \mathrm{~s}$ from ghost finite volumes. As mentioned earlier, the derivatives for the constraint equations are computed for the ghost finite volumes of the process. The results from the computations are stored into the corresponding host finite volumes. Thus, all the information needed for performing the Gaussian elimination can be obtained without inter-process communications. The equation decoupling by using Gaussian elimination is performed on individual processors. Our experience has shown that the computational time for Gaussian elimination is minimal. Therefore, for those problems containing many constraint equations it is worthwhile considering Gaussian elimination.

\section{Scalability test}

Different criteria have been proposed to analyze the scalability of parallel codes for different applications. In oil simulator application, two methods are generally used to represent the scalability of the parallelization:

1) Fixed size problem and

2) Problem with varying size proportional to the number of the processors. 
The speedup is one of the indices to express the efficiency of the parallel code for a fixed size problem approach and it is defined as

$$
\text { speedup }=\frac{t_{1}}{t_{\mathcal{P}}}
$$

Where, $t_{1}$ is the total time needed for computation with a single processor and $t_{P}$ is the total time required after the problem is divided into $\mathrm{P}$ pieces and solved on $\mathrm{P}$ processors.

For varying size problems, the ideal parallelization occurs when the real computation times are unaffected during the scale up. However, the cost for cross-node communications grows when the size of the problem increases, and as the result, the real computation time increases as well. The time difference between the ideal and real performance can be used to identify improvements that can be made in the implementation of the parallel code. For theoretical maximum speedup where the communication time is zero, the speedup is close to P. However, in reality the speed is less than $\mathrm{P}$ due to the penalty from communication cost, load imbalance and the algorithm (Karniadakis and Kirkby, 2003) employed.

\section{Parallel Performance}

Performance of the parallel simulator for a number of domains with the thermal compositional simulator is shown in Figure 8.3. The performance was not optimized - but the speedup appears reasonable. Biconjugate gradient linear solver was used in all applications. 


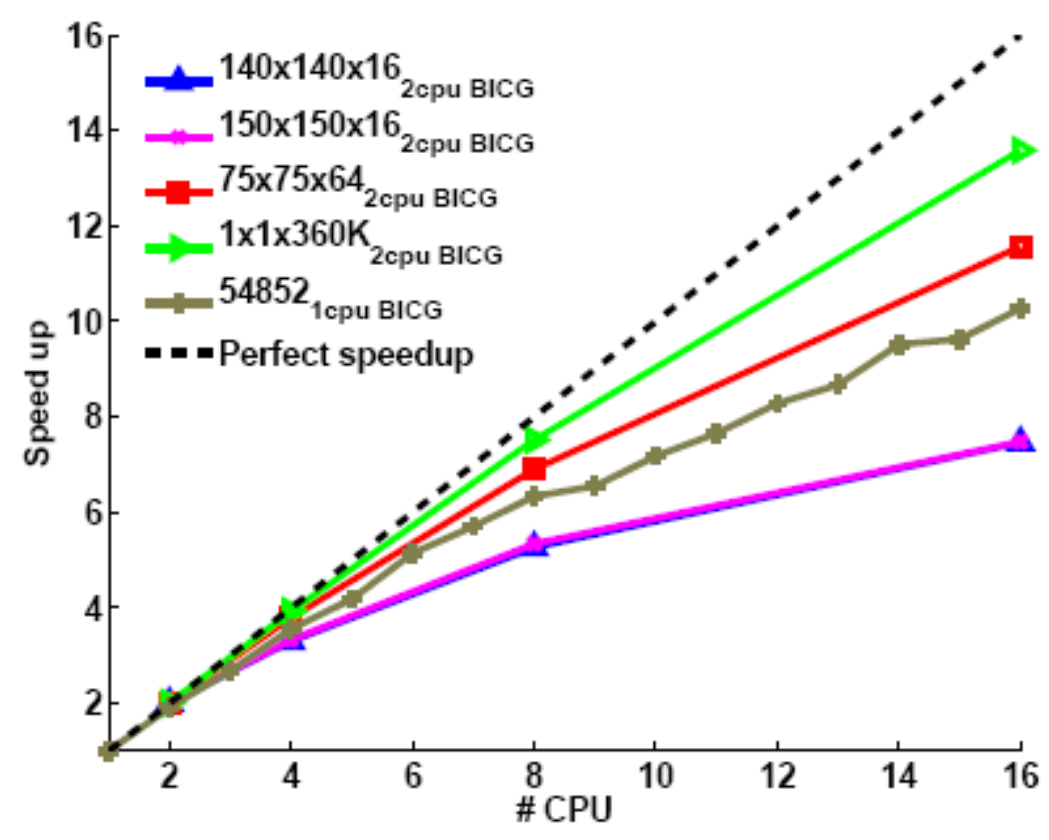

(b) speedup

Figure 8.3. Parallel speedup of the thermal compositional simulator using the bi-conjugate gradient linear solver.

\section{Chapter Bibliography}

S. Balay, K. Buschelman, V. Eijkhout, W. Gropp, D. Kaushik, M. Knepley, L. C. McInnes, B. Smith, and H. Zhang, 2007, PETSc Users Manual, Argonne National Laboratory, 9700 South Cass Avenue Argonne, IL 60439, 2.3.3 edition, May 2007.

Karypis and Kumar, 2004, A Software Package for Partitioning Unstructured Graphs, Partitioning Meshes, and Computing Fill-Reducing Orderings of Sparse Matrices, University of Minnesota, Department of Computer Science Army HPC Research Center, 4.0 edition.

Karypis et al., 2006, Parallel Graph Partitioning and Sparse Matrix Ordering Library, University of Minnesota, Department of Computer Science / Army HPC Research Center, 3.1 edition.

M. A. Heroux and J. M. Willenbring, 2003, Trilinos Users Guide, Sandia National Laboratories, Albuquerque, New Mexico 87185 and Livermore, California 94550, 8.0 edition, August 2003.

G. E. Karniadakis and R. M. Kirkby II, Eds., 2003, Parallel Scientific Computing in C++ and MPI, Cambridge University Press, 2003. 


\section{Chapter 9. Generating Fracture Networks, Gridding and Creating Simulator Input Files}

\section{Table of Contents}

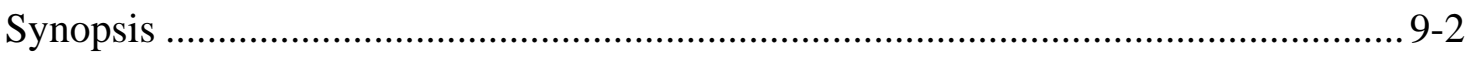

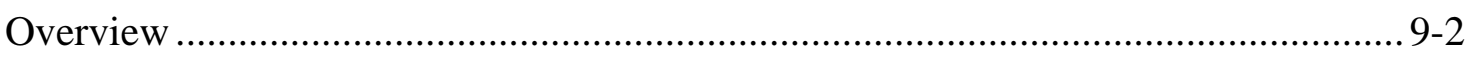

Fractured Network Characterization Packages …………………………...................... 9-3

Gridding Packages................................................................................................. 9-4

Assembling Simulation Input File.............................................................................. 9-4

Simulators................................................................................................ 9-5

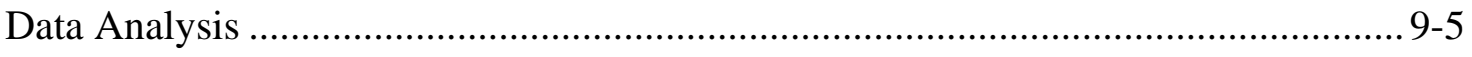

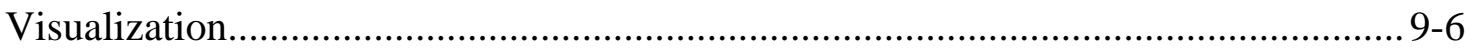

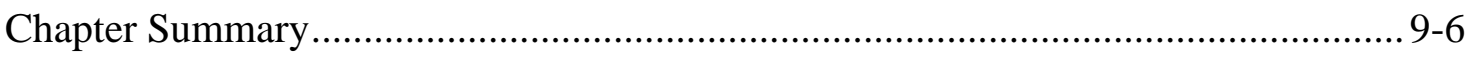




\section{Synopsis}

Simulating fractured reservoirs and predicting oil recovery requires integration work. The integration work should include geologic knowledge, petrophysical properties representation, well modeling and well history input. Most times, data required for this integration are scattered in various models and programs. One of the main objectives of this research program is to bring about the integration of the geologic fracture network model and reservoir simulation input file.

\section{Overview}

A workflow tool was created in this project with the following features:

- Treatment of fracture only reservoir or reservoirs with fractures and matrix.

- Meshing the domain created. Meshing was discussed in detail later in this chapter.

- Assigning properties to the control volumes and/or elements created during the meshing exercise.

- Creating a simulation input file.

- Assigning wells and well operational parameters.

- Describing well production histories.

- Executing the simulator with the given input file.

- Generating output data for production analysis and for visualization.

- Visualizing the results.

A schematic of this workflow is demonstrated in Figure 9.1. 


\section{Fractured Network Characterization Packages}

“FracMan Reservoir Edition” (FRED) is a sophisticated general program for importing a number of geologic inputs into a geologic model to create fracture sets characteristic of all of the measured data. FRED was used in this project as the primary fracture generation tool. These fracture sets may be operational in a Type I reservoir environment or a Type II reservoir situation, where the fracture sets are embedded into a reservoir matrix. When creating reservoir simulation input files using the sophisticated fracture network, both these aspects need consideration.

Multiphase DFN Simulation Work Flow

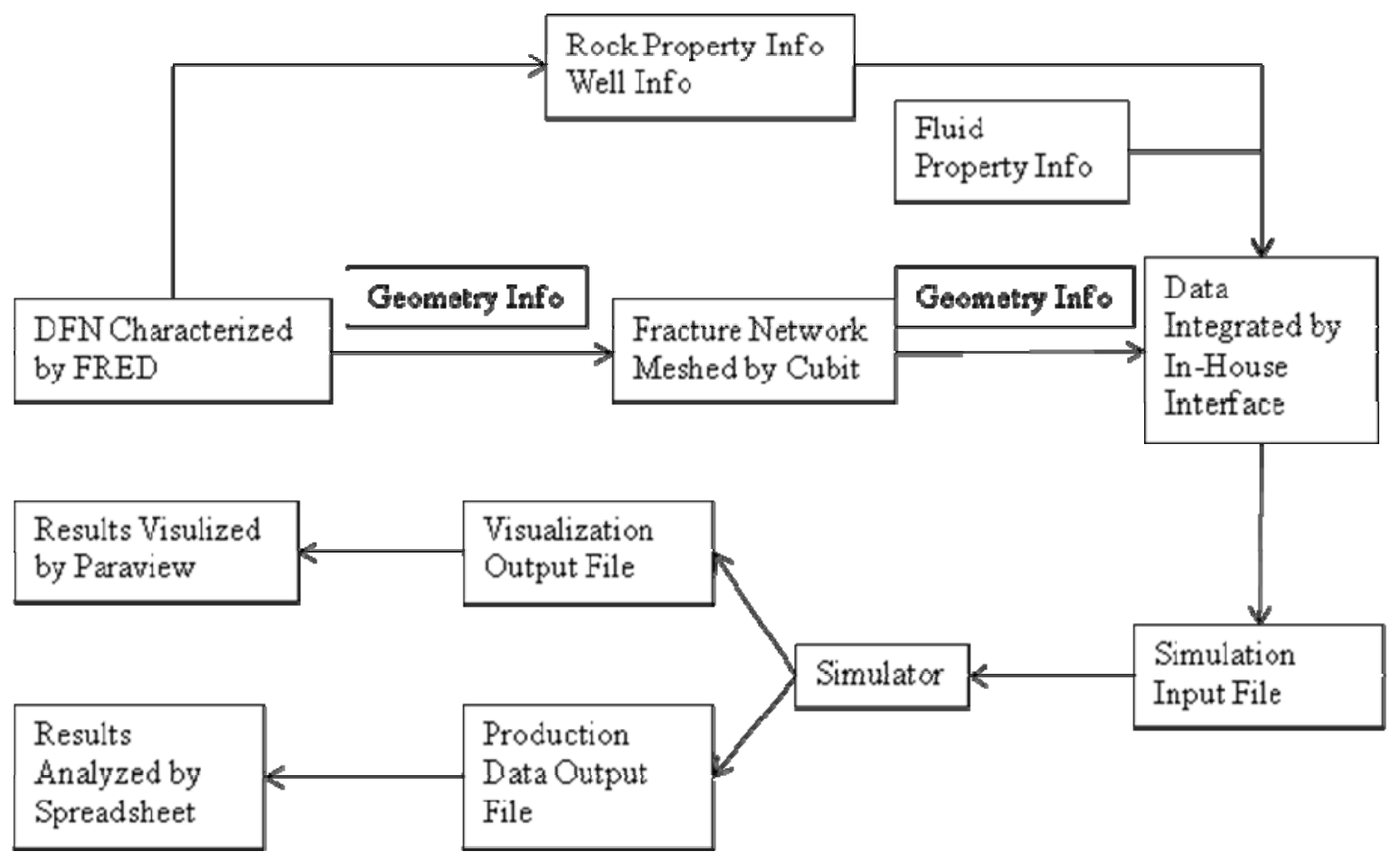

Figure 9.1 An example of multiphase DFN simulation workflow

PETREL is another powerful petroleum reservoir characterization package. Since the 2007 version, some FRED functions are integrated into the PETREL package. The PETREL package is used in this workflow to characterize the structural fault and to generate corner point grid 
blocks. These grid blocks could be combined with FRED generated DFN models for the purpose of permeability and intensity upscaling. The upscaled model could be simulated by conventional finite-difference simulators such as ECLIPSE and IMAX.

\section{Gridding Packages}

Domain meshing is a big concept for reservoir simulation. In order to calculate the fluid flow through the porous media, modeled reservoir domain must be meshed based on numerical discretization method adopted. Today, the trend in simulator development is to separate the meshing part from the flow calculation (simulator) part, and use the output from the meshing part as input for the simulator. The meshing part is normally done by meshing software. Through this approach, one simulator should be able to couple with different meshing programs.

As mentioned in the last chapter, in this fractured reservoir integrating workflow, the PETREL package is adopted for conventional finite-difference meshing with an upscaled DFN model inside of grid blocks,

The control volume finite-element discretization method (CVFE) applied in this research has the advantage of easy handling unstructured geometry and a higher order of accuracy. The finiteelement mesh software packages include CUBIT from Sandia National Lab, HyperMesh from Altair Co., RoseMesh from Golder Associate, and MeshMaster from Golder Associate.

\section{Assembling Simulation Input File}

PETREL has a very mature package to put production/injection history and petrol-physical data into the ECLIPSE input file. For the workflow of simulating upscaled DFN modeled fractured reservoir by conventional finite-difference simulator, PETREL can have the work done. 
For unstructured grid CVFE simulation, there is not any commercial software that could be directly applied in assembling the simulation input file. In this work, an interface (different from Chapter 8) has been developed to assemble the meshed unstructured DFN geometry with wells and other petro-physical information to form the input data file. This interface package is designed as modulated too. It has the very flexible capability to assemble different CVFE simulation input files, such as: type I and type II fractured reservoir, 2-dimensional or 3dimensional, 2-phase or 3-phase, and so on.

\section{Simulators}

The simulators integrated in this fractured reservoir study include both conventional finitedifference simulators such as ECLIPSE and the finite-element based unstructured simulator of CVFE. The finite-difference simulator is used for upscaled DFN modeled fracture reservoir studies. The single porosity models were applied on type I fractured reservoir and the dual porosity models were used on type II fractured reservoir upscaling calculations through this finite-difference simulator.

For unstructured DFN modeled fractured reservoir, the CVFE simulator is adopted for direct DFN characterization simulations.

\section{Data Analysis}

A postprocessing code has been developed through this integrating workflow for the CVFE simulator. These postprocessing efforts include the pressure or saturation distributions; injection or production analysis by phases, by independent wells or by total reservoir performance. The 
postprocessed data could be directly imported by a coded spreadsheet file and all kinds of analyzing curves and figures are generated by that coded spread sheet file automatically.

\section{Visualization}

Other than the in-house visualization tool in Chapter 8, ParaView is adopted as the standard visualization package for this fractured reservoir integrating workflow.

ParaView is an open source, multiplatform application designed to visualize data sets of sizes varying from small to very large. ParaView runs on distributed and shared memory parallel as well as single processor systems and the successful testing has been reported. Today, ParaView development continues as a collaboration between Kitware, Sandia National Labs, CsimSoft, Los Alamos National Lab, Army Research Lab and others.

\section{Chapter Summary}

As shown in Figure 9.2, a multiphase, multidimensional fractured reservoir simulation workflow has been developed through this work. This workflow includes accurate simulating of finiteelement based unstructured DFN modeled type I and type II fractured reservoir and their upscaled studying through conventional finite-difference based simulator. To assure this fractured reservoir integrated workflow to be seamless, the modules created led from characterization through the postprocessing analysis such as the visualization. Developing and integrating an accurate, efficient workflow to model and simulate the multidimensional, multiphase, multiscale fractured reservoir was a significant contribution. 


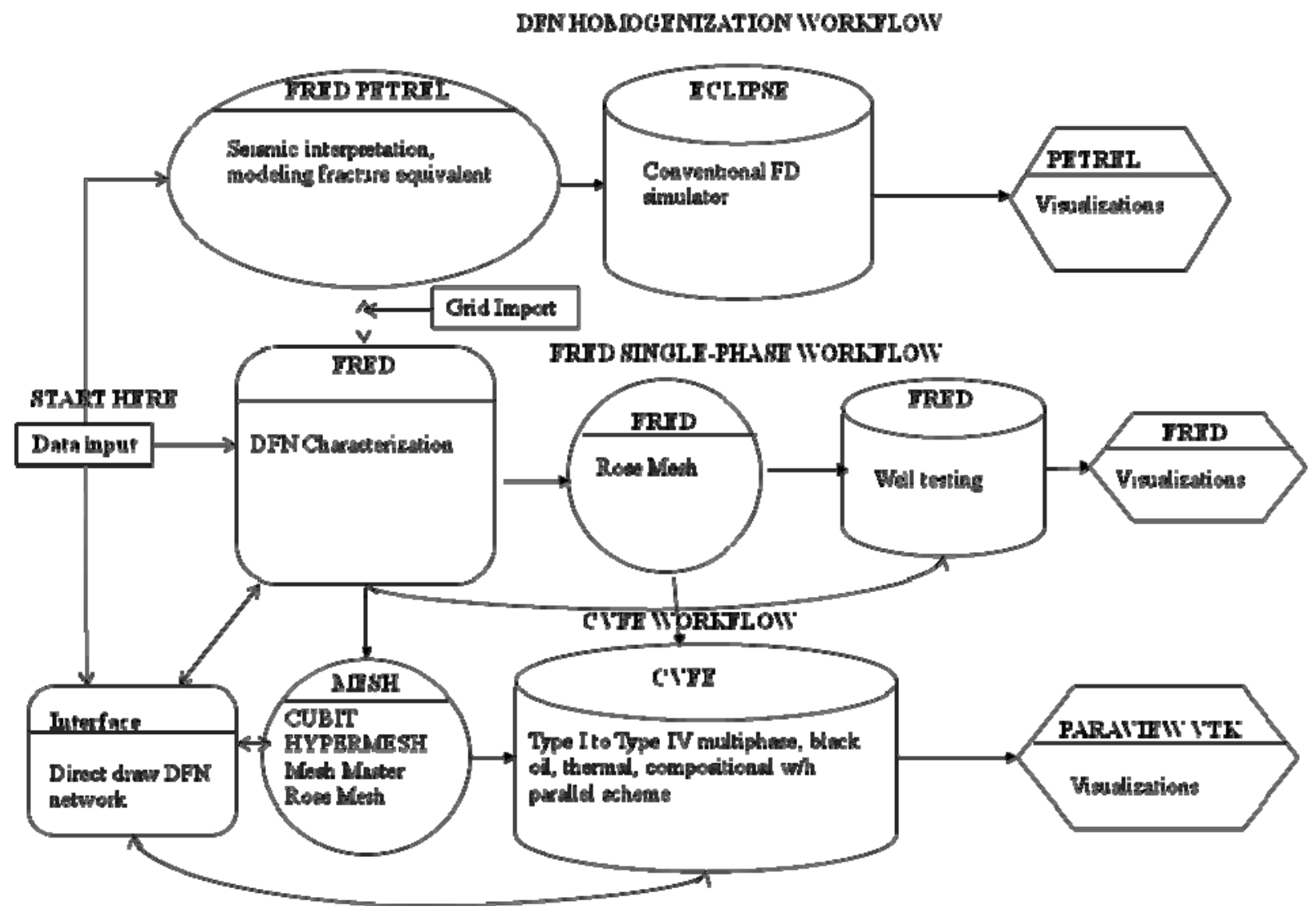

Figure 9.2 Illustration of workflow on fractured reservoir simulation 